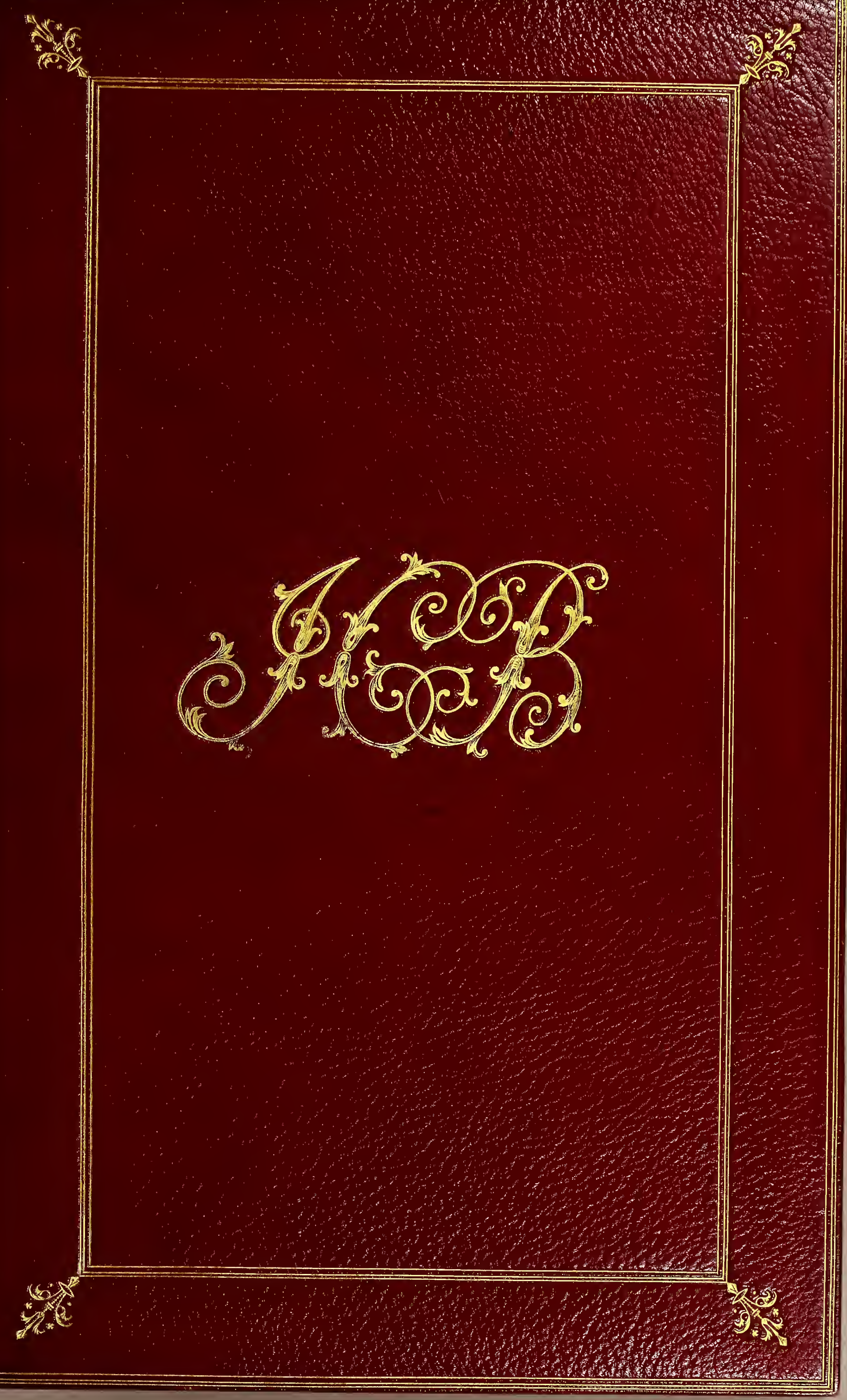




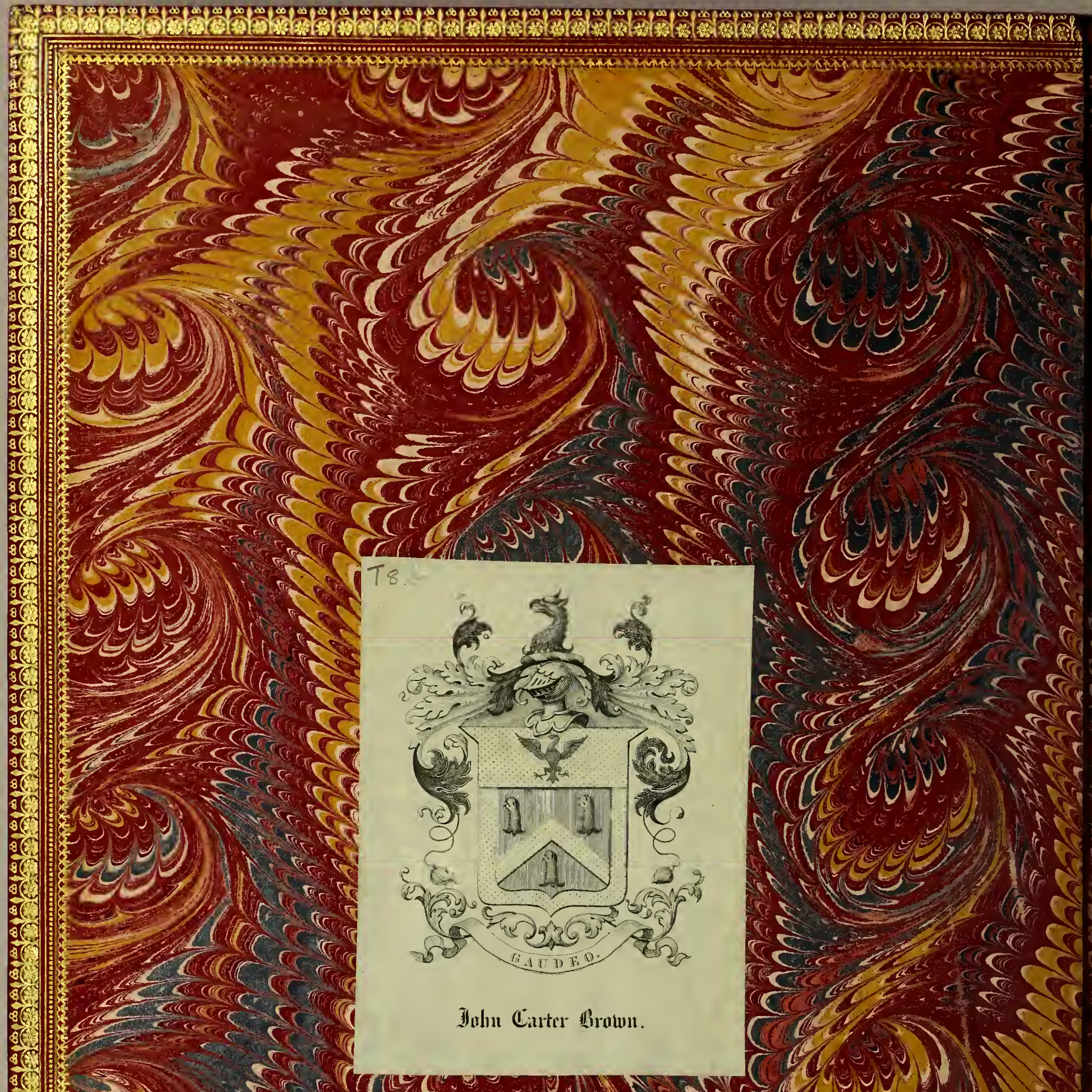

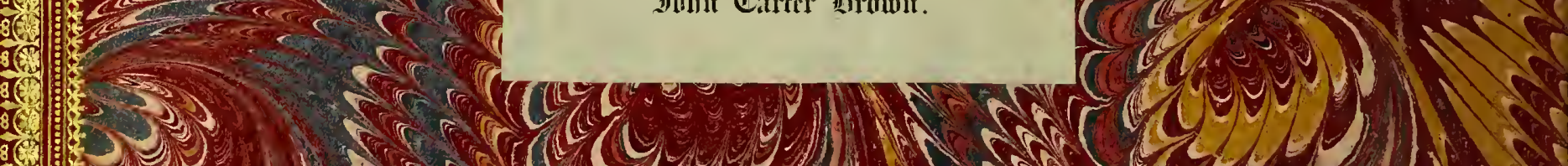
(E) I ( (

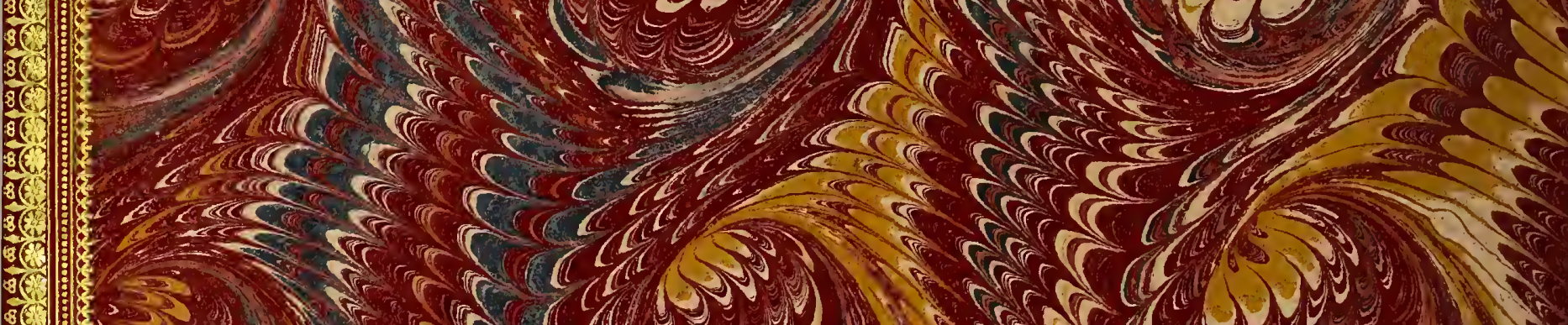
-

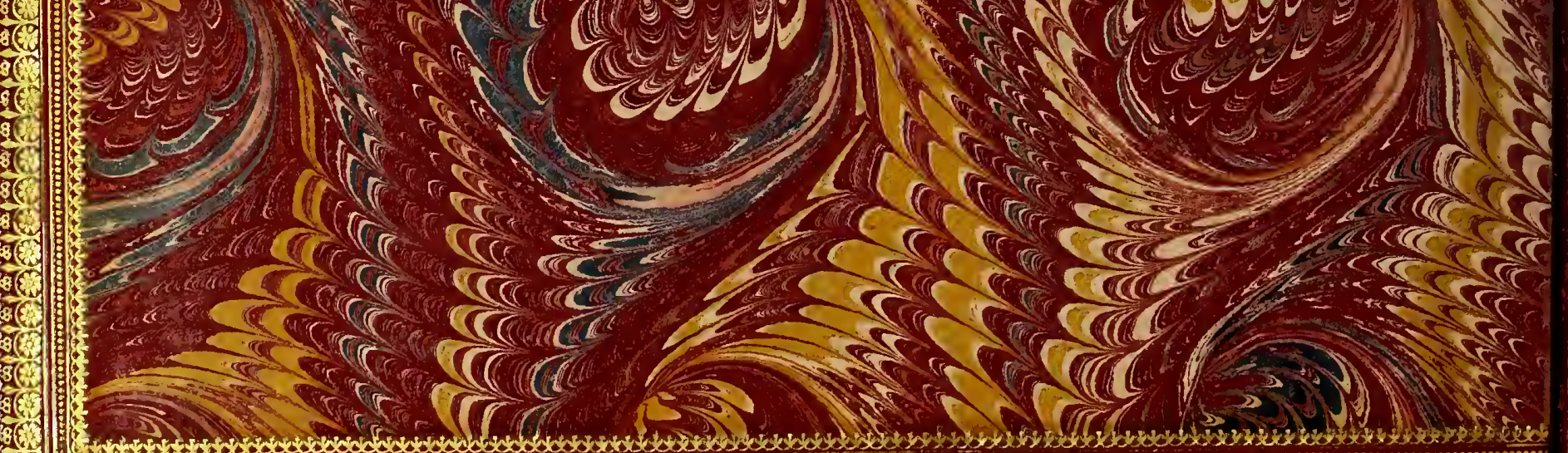




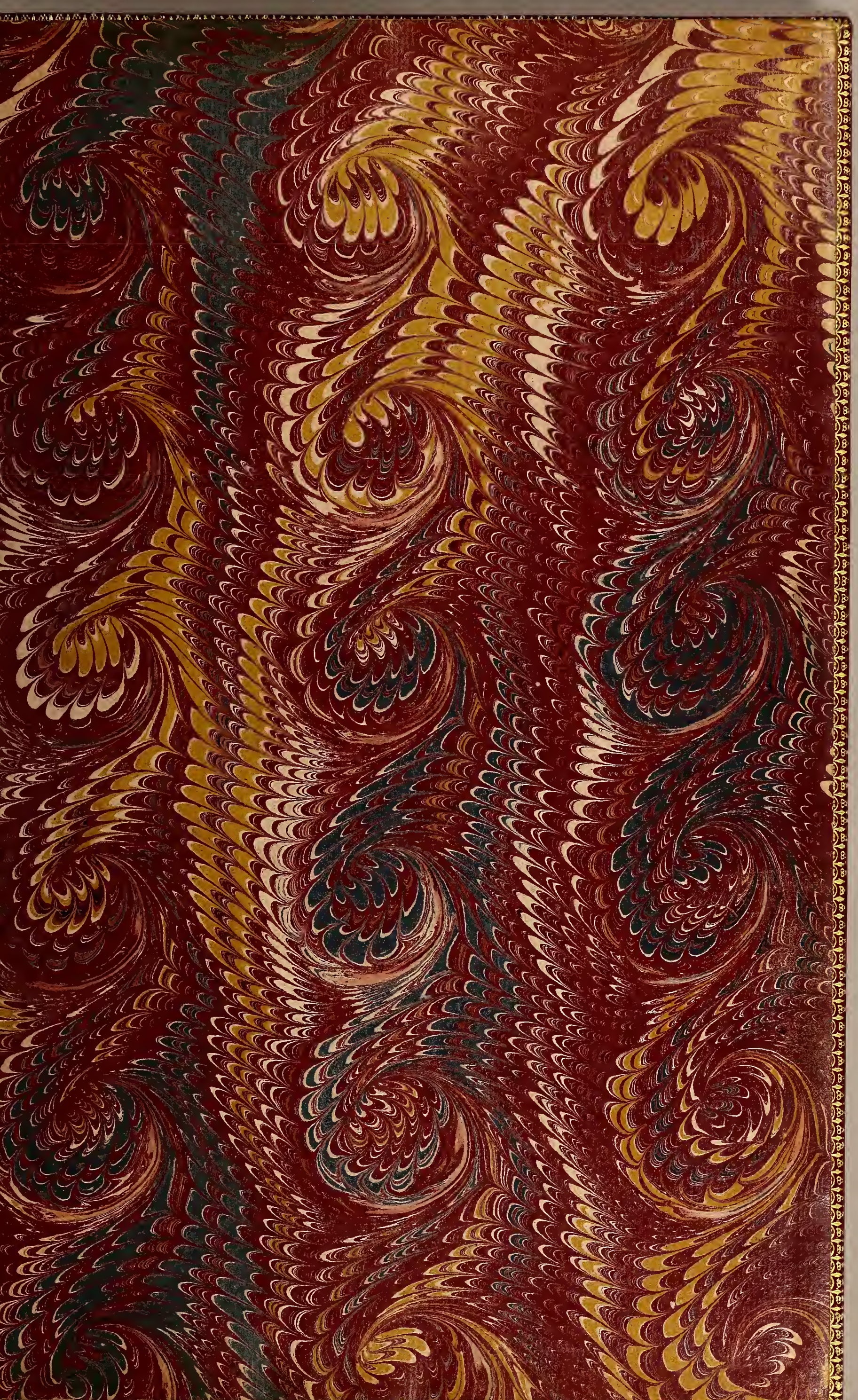




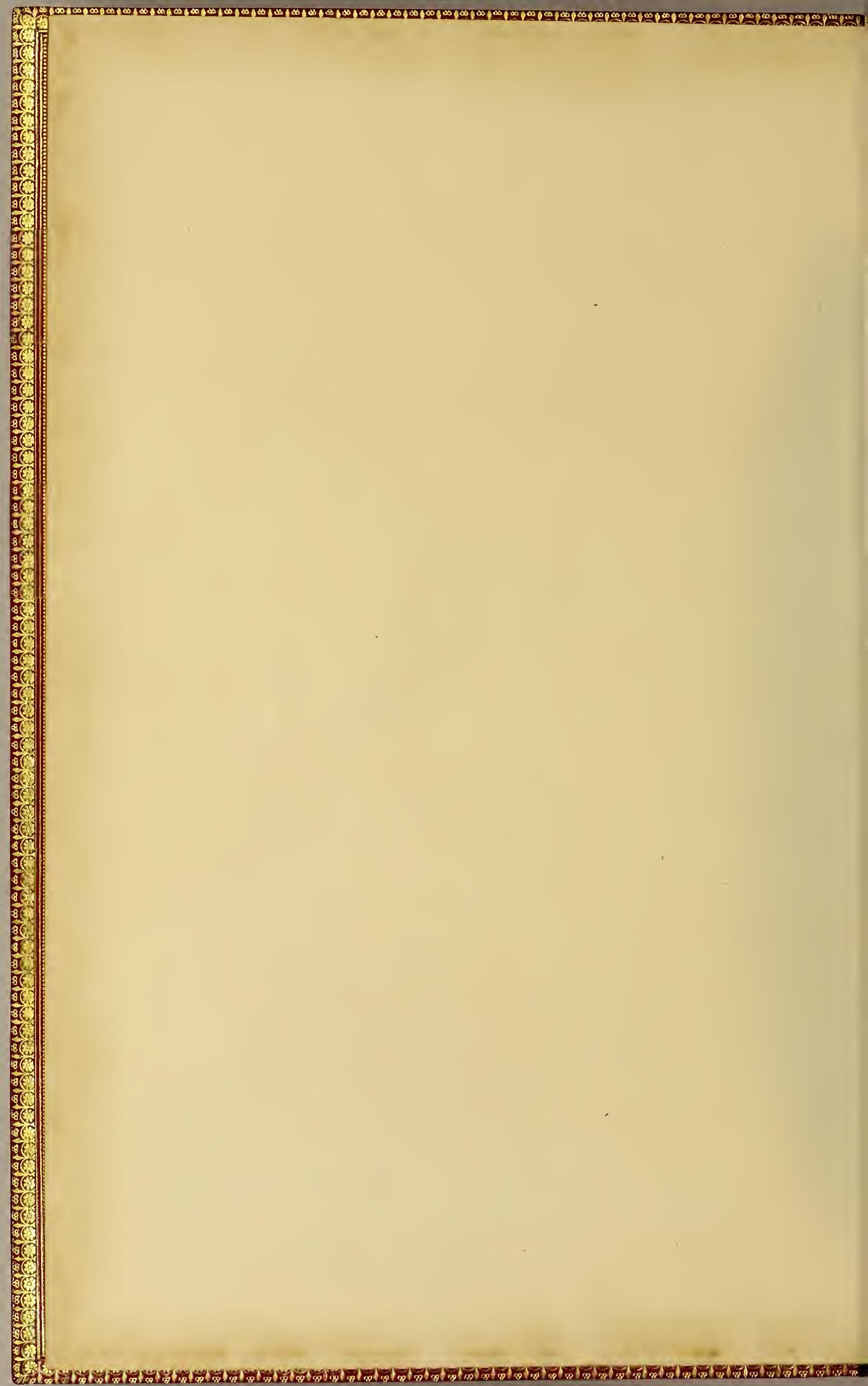




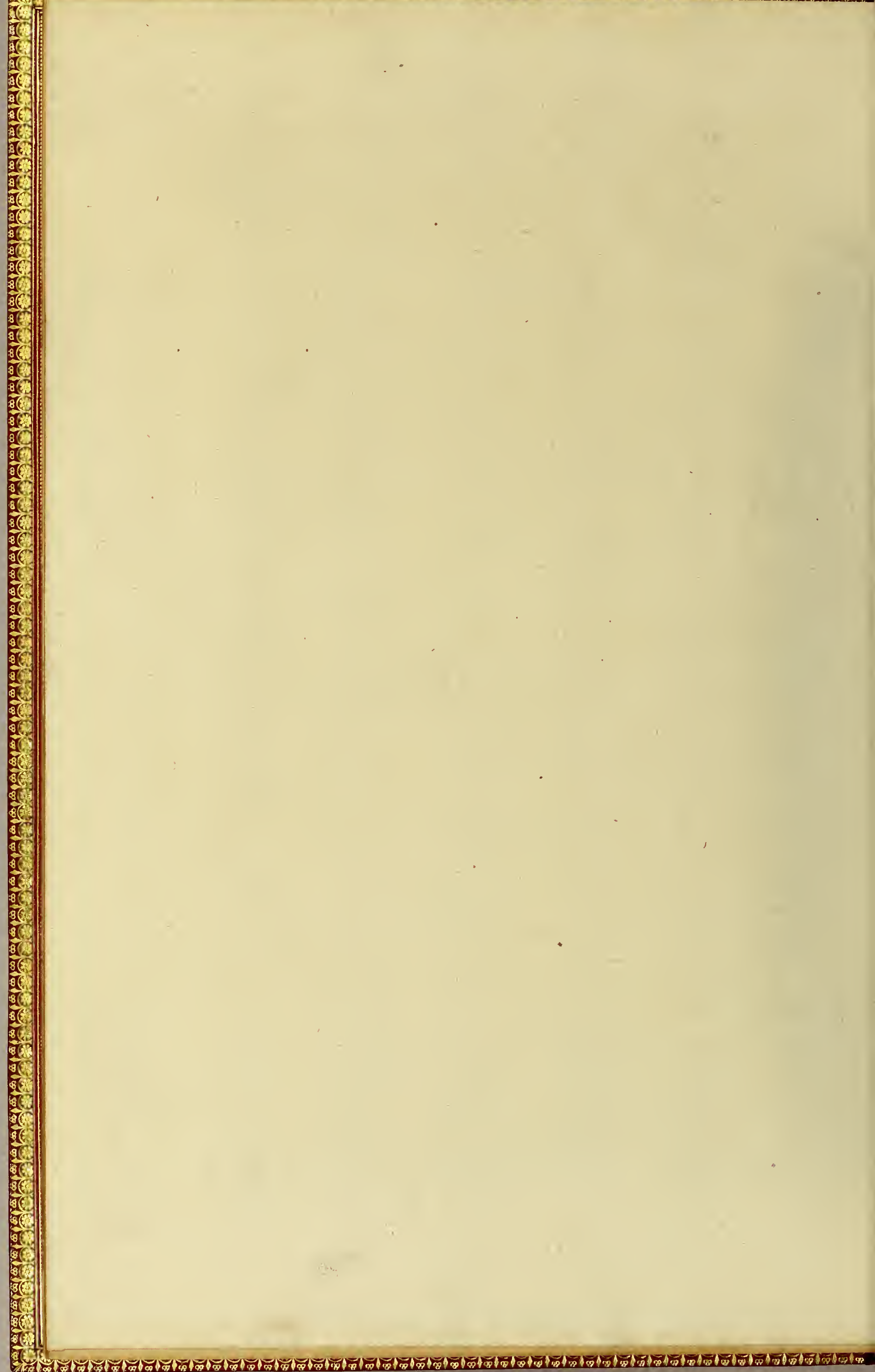




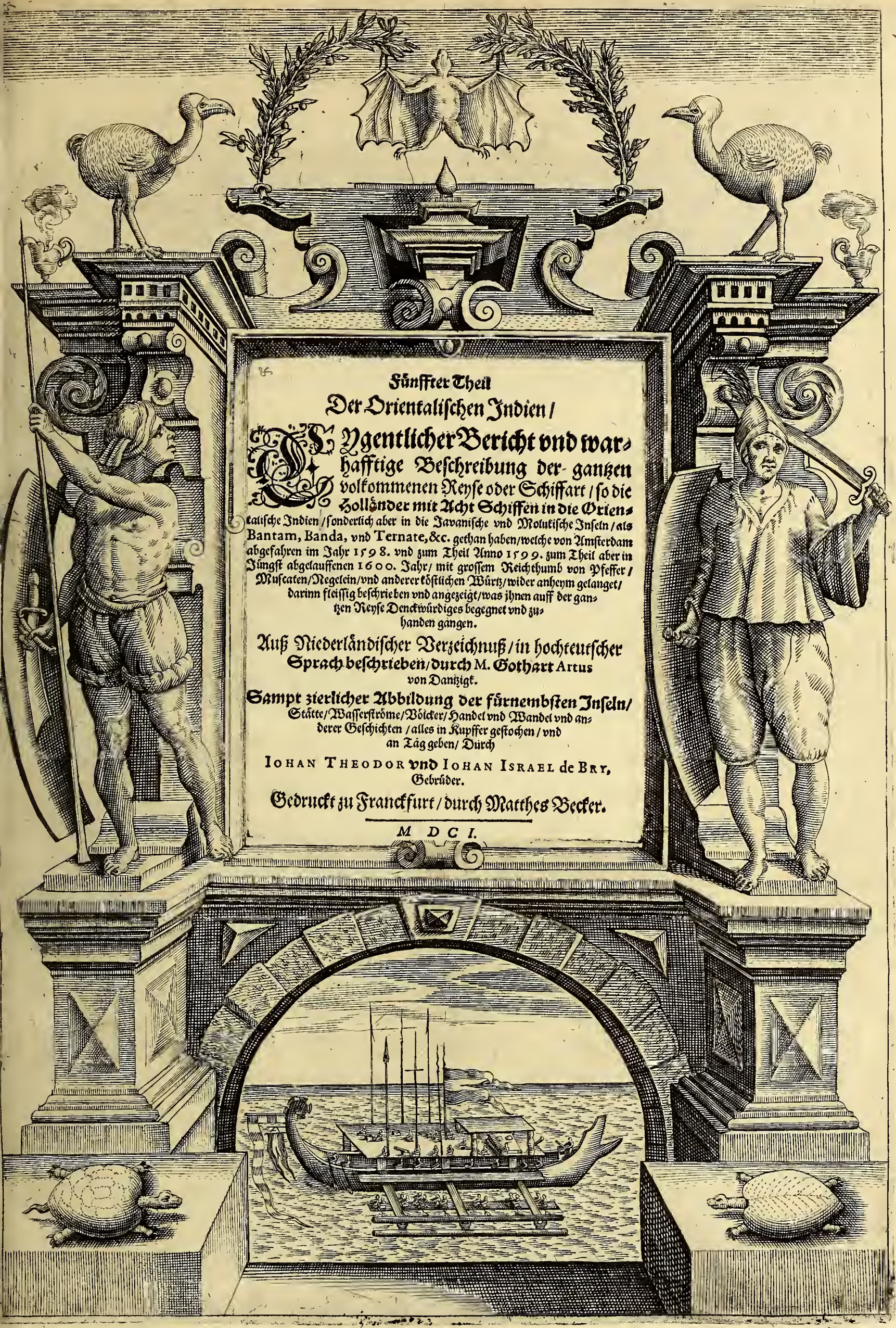




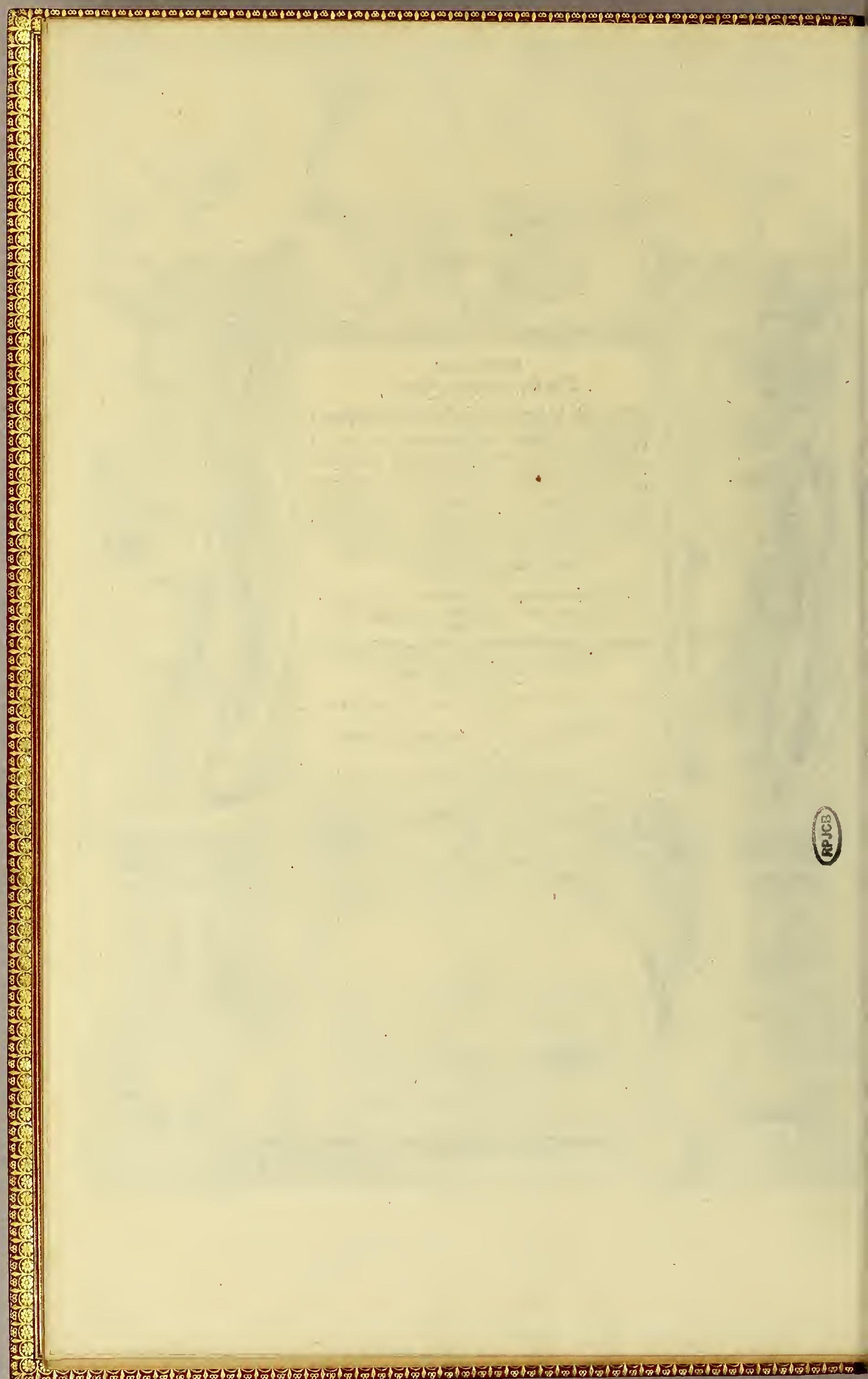




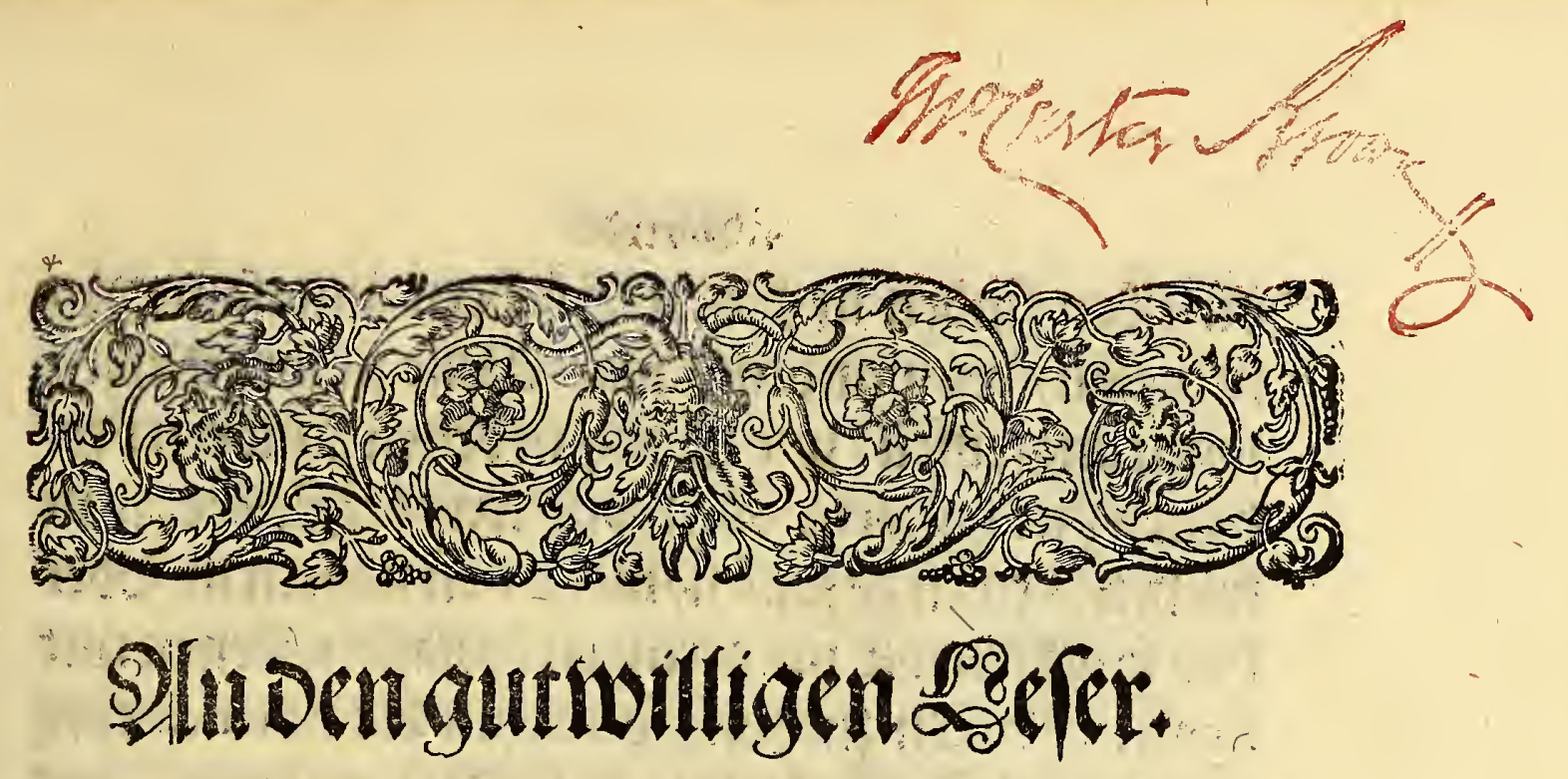

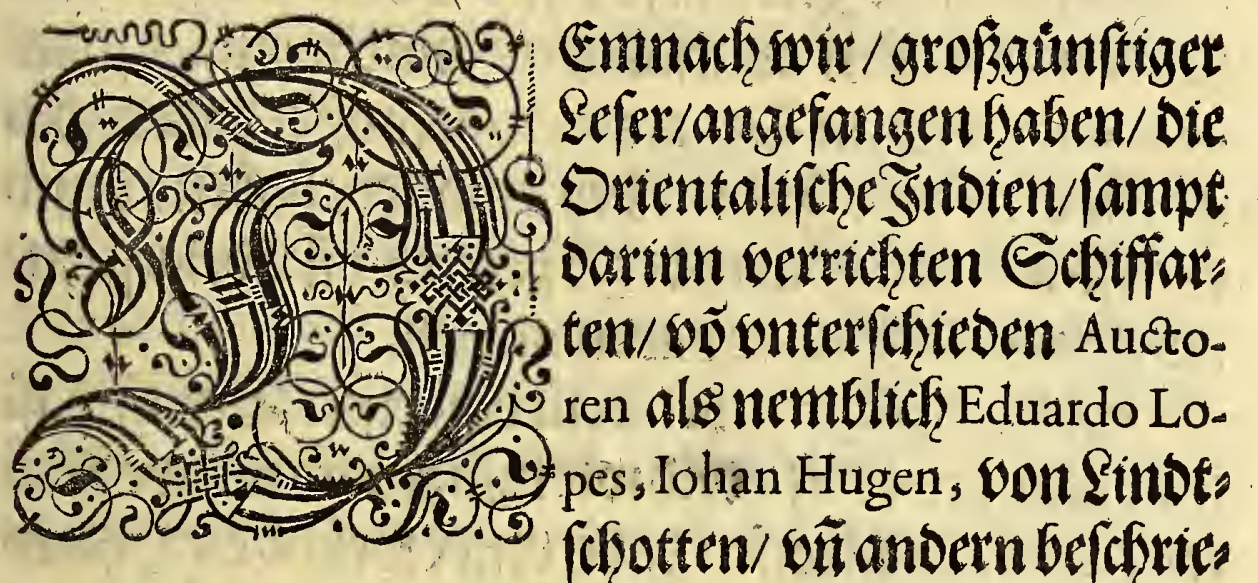
ben/m Druce werfertiget an Sag zu geben/2hth etlis cher maffen wernterctet wie biefelbe bey wielen lieb

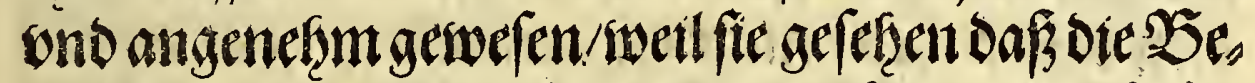

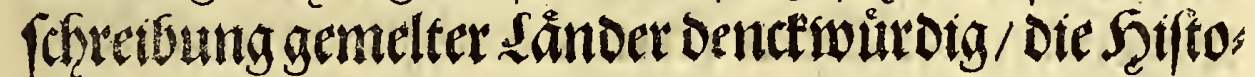
rien Der Schiffarten furtherwetlig/ ond Die eygentliche Fưrbilloung Der fremboen $\mathfrak{B o l k f e r} /$ iferer Sitten / Fleysung vond andern Sertemonien anmufetiglenn/

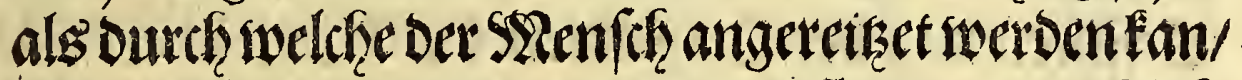

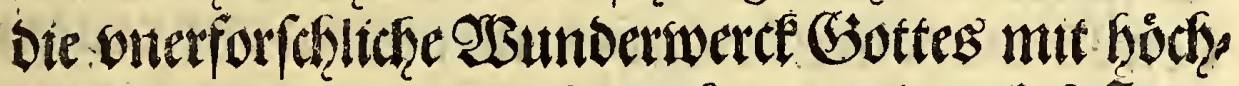
fer Zerwunderung zu betracteten/ ono in Erfeñung

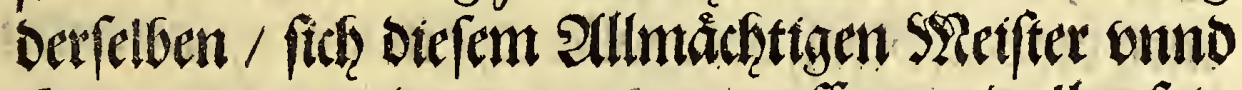
SEern gank ond gar zu onterwerffen/ond alles fein

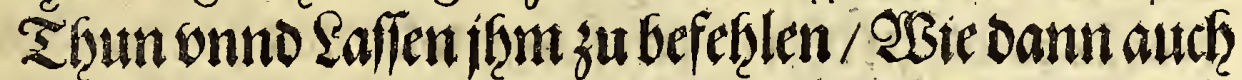

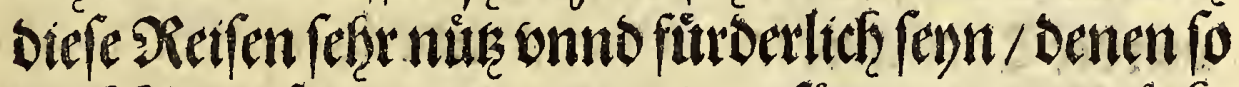

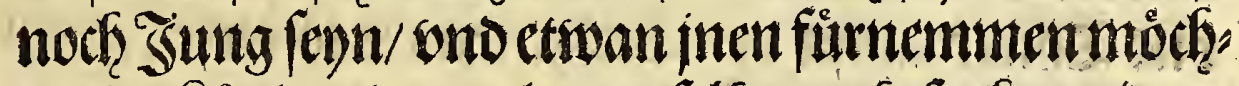
ten bie Lànoer bermal eins felber zubefucten / Denn

$$
x \text { in oiefled }
$$


Borreters

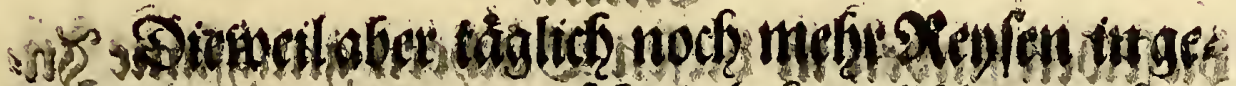

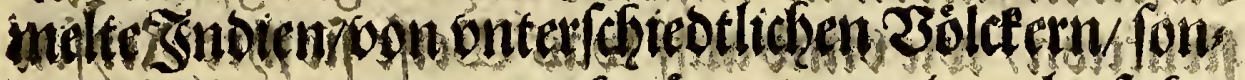

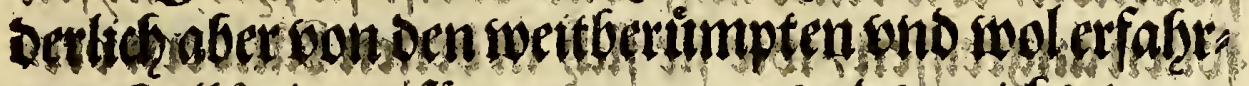

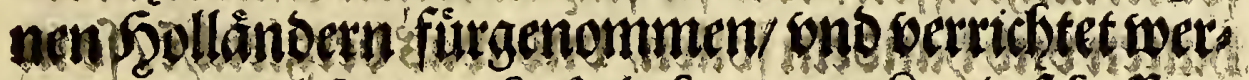

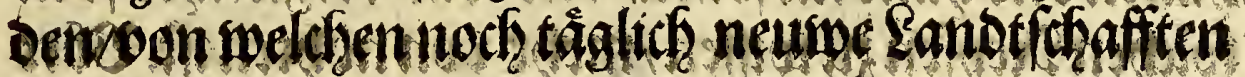

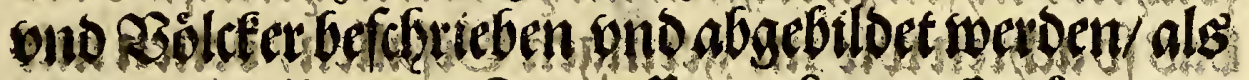

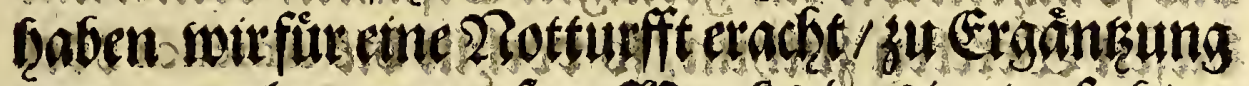

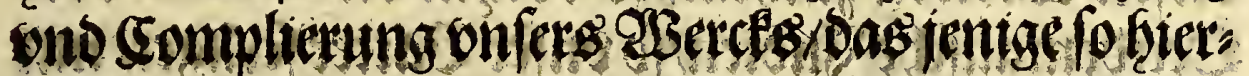

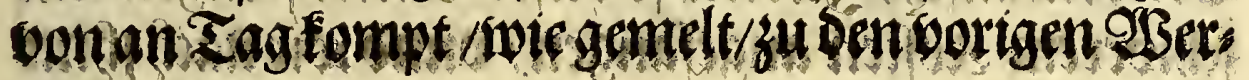
cten $u$ ufiggen.

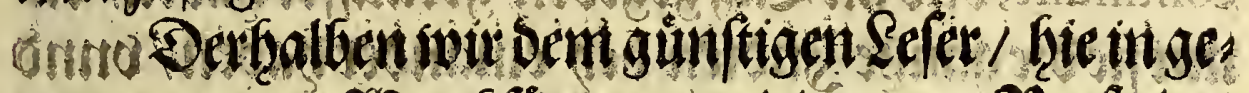

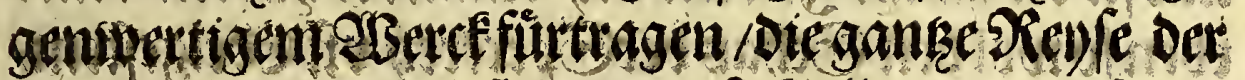

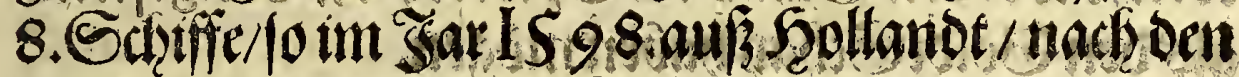

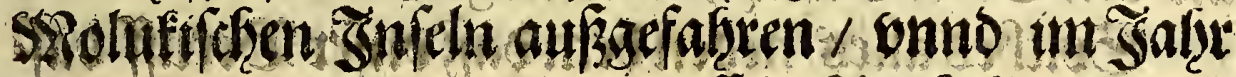

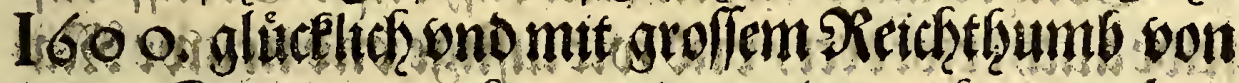
veler Spccrennach einanoer wotoer anfenm gelan get fondot/ mitgroffem2us ono Srolocfungoeren/

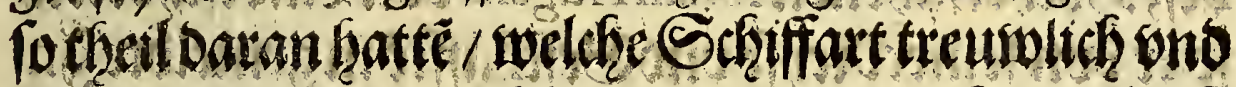
fleifigmit allen wombftanden/von enem fo derPied/e mitbey gerwofnet bat/ befortedn worden / onno in Shollonot inifare Sprads mit etlichen wartiaffigen

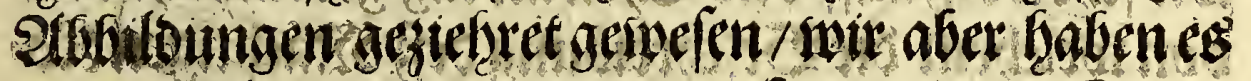

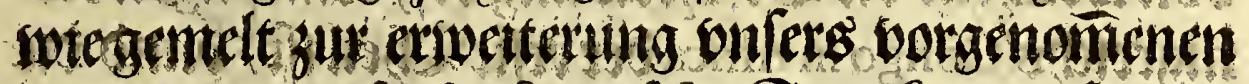

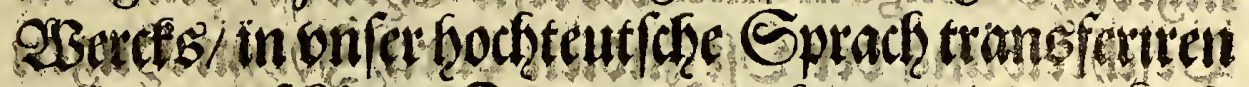
Iafenmit fhonen figurengesteftet ono pen \&ieb. fancufremboen ono wartofftiger siftorien zugefal

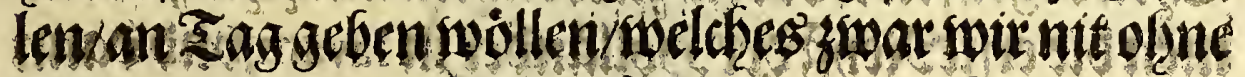

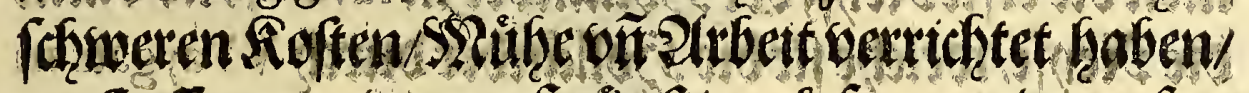

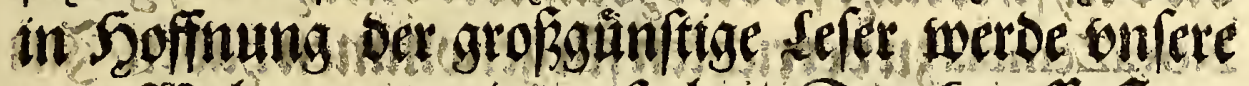

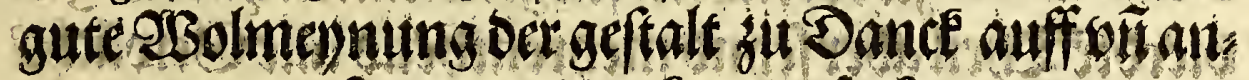

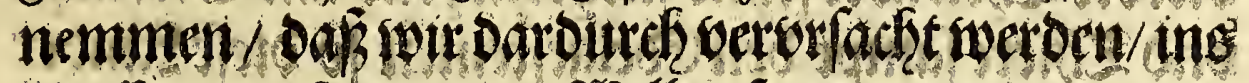

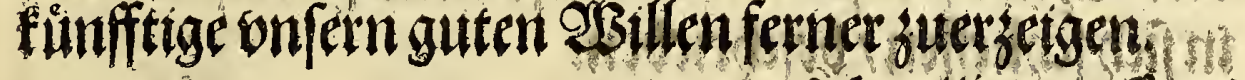
$x$ ii $x$ Eins 


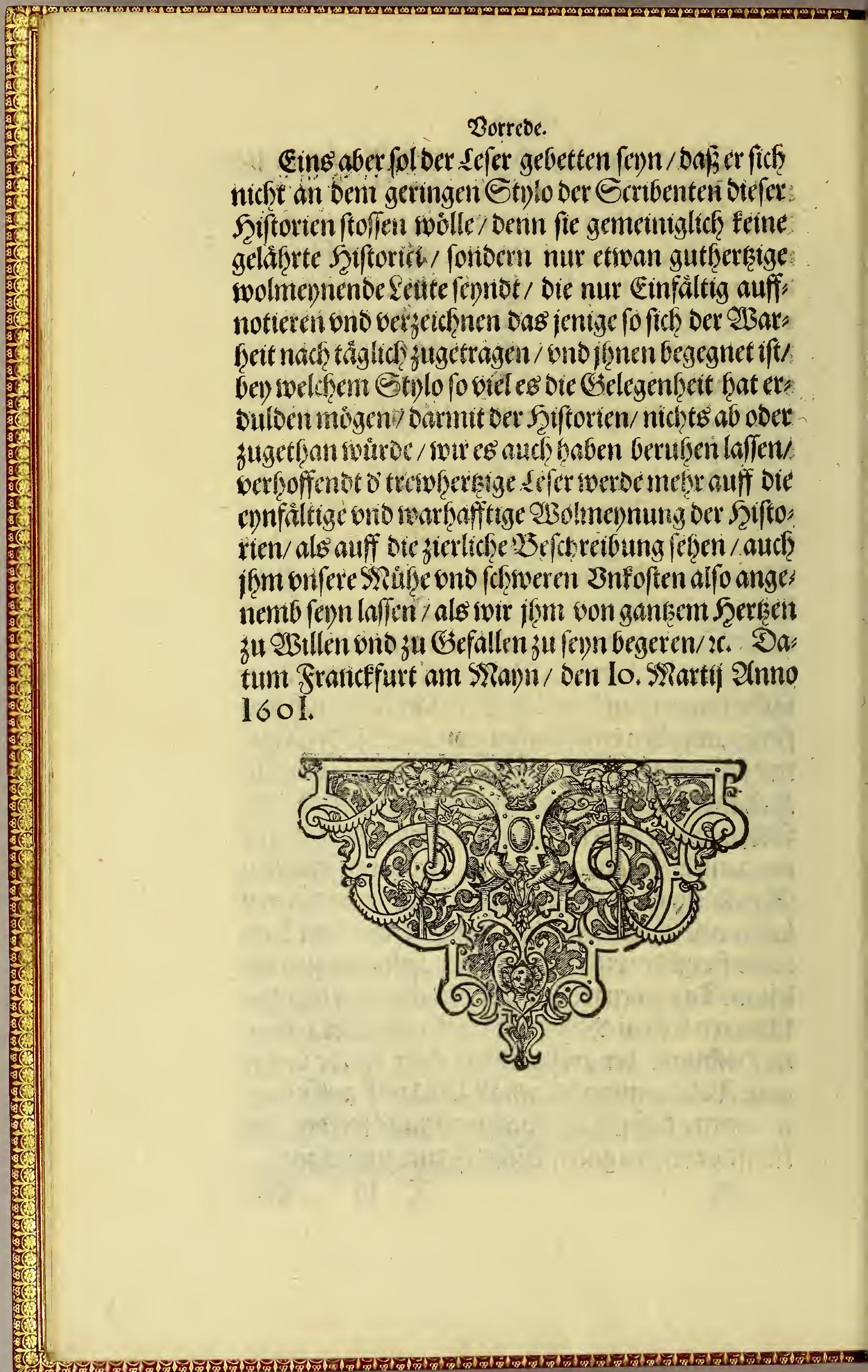



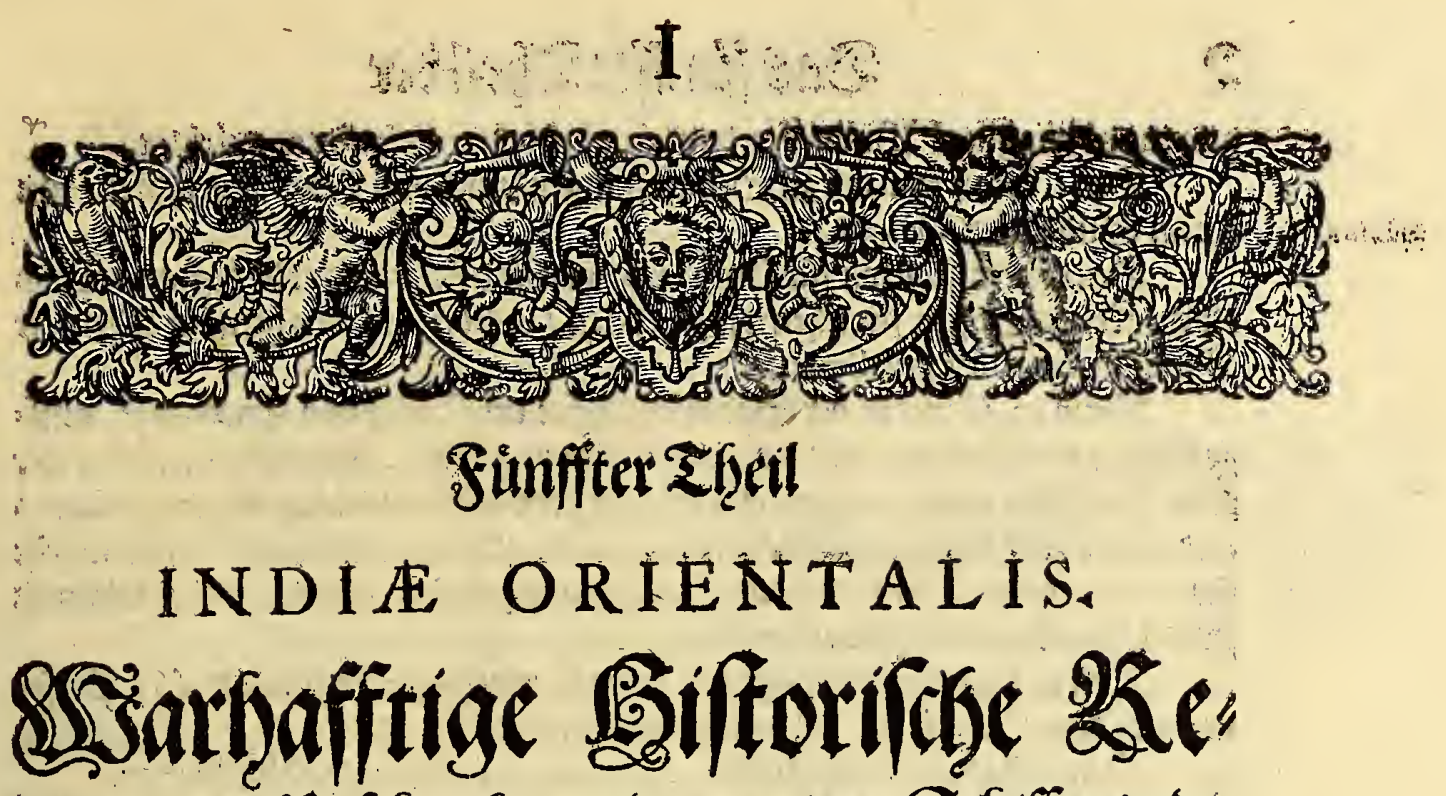
lation ono $\mathcal{B}$ effretbung bet ganken Schiffart ober

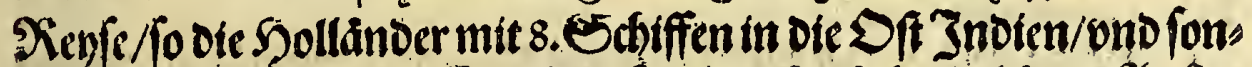

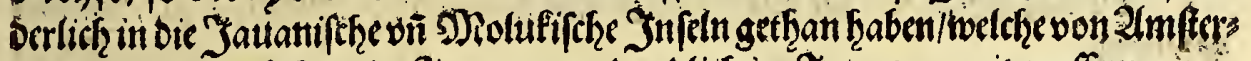

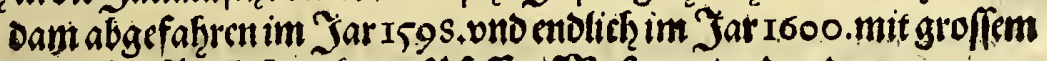

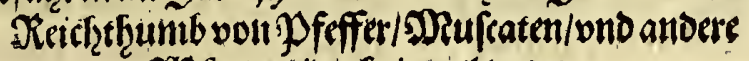
WBirkerswiber Eerimgelanget.

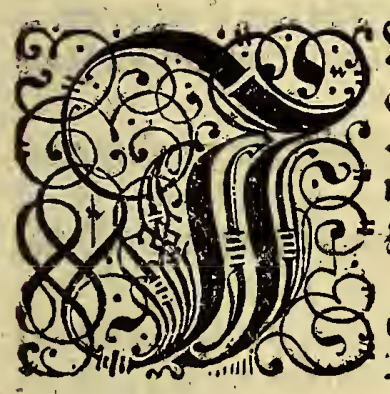

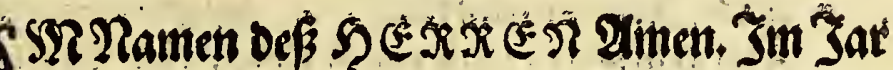

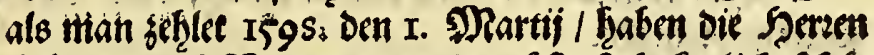

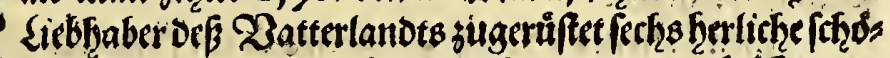

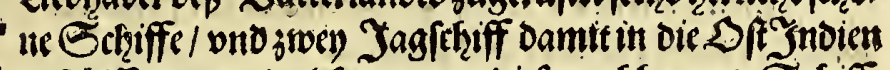

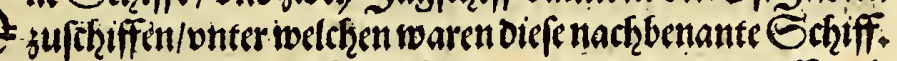
Erfttith kin Ecthiff genant Mauritius, auff wet chent war Der Zlomiral / lacob Cornelius Neck bon Um/ferdam/ Der Ethiffmann aber hiteß mit Dem Namen

Gouaert lanfz.

Das anerr Schiff hies Amfterdath auff welthent wat der Diceztomirali Wybrant Warvvick, von 2 mfteroant ṽ̃ Schiffmañ Cornelius lans; Forruiin. berts Mau.

Def oritten Sehiffs mitnamen Holland, Erfiffimann war Simon Lam-

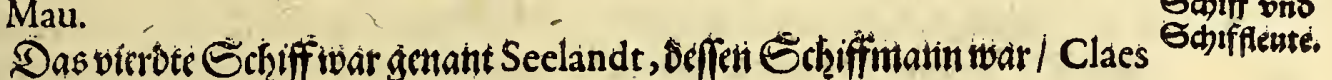

lans Melcknap. Bruiin $_{2}$

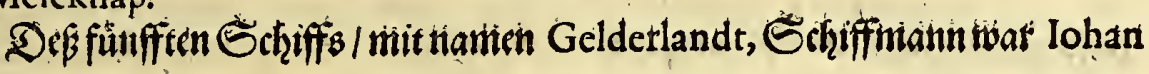

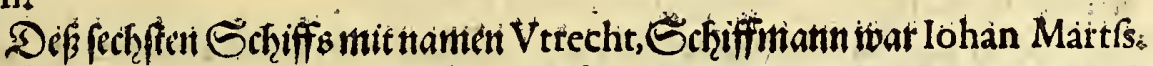
Dasfieberide war das groffe Jag/chiff/mit namen Vrieslandt genant/ bnno beffon Ëchiffithanutivar loan. Cornelifs.

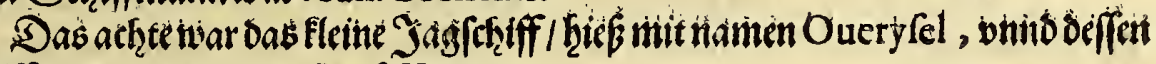
Echiffintantribar simon lanis Hoen.

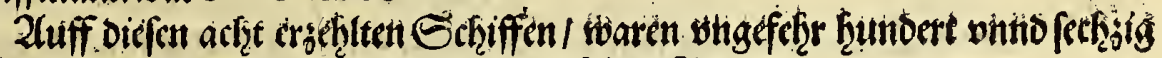
Perfonen/weletre gentulfert worderi/Den 9. Tag STartii.

Außfaber Den 13. SPart. fennt wir '̆t

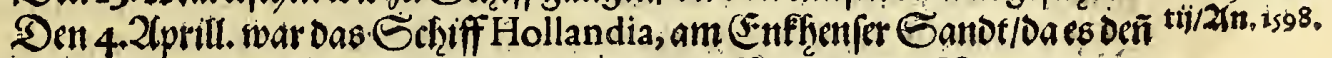

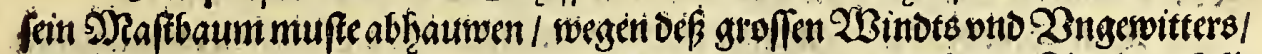
woir fentrot aber beil 22, bito gen Texel anfommen/ vno bafelbft acht Iaglang fille X ... geligen 


\section{Orientalifchen Snbien.}

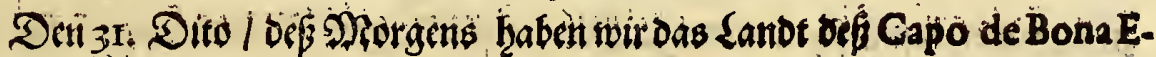
Sperancasefethen.

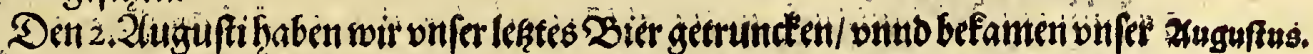

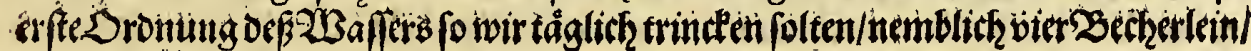
vno orein Seckęerléti mit $2 B$ ein.

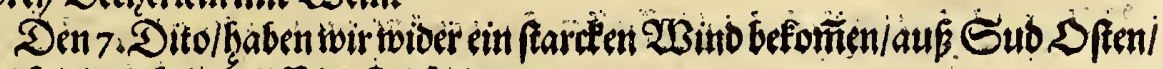

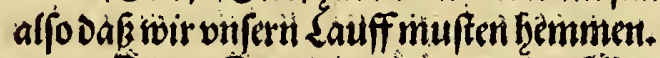

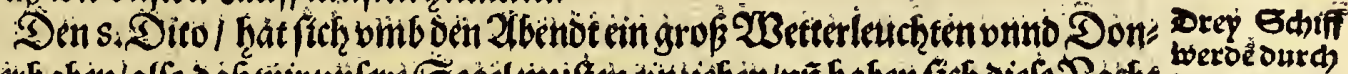

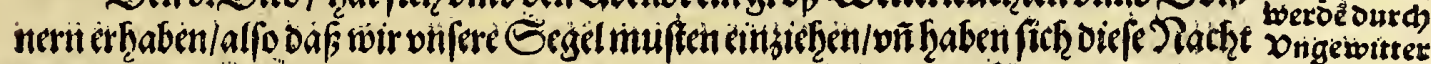

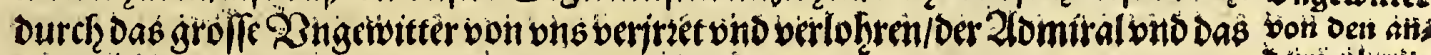

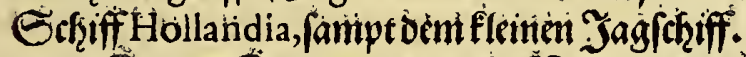

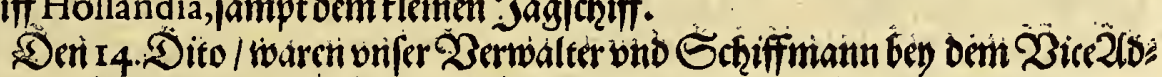

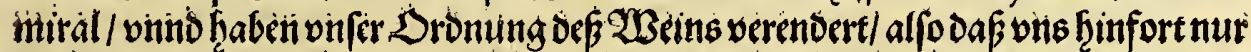

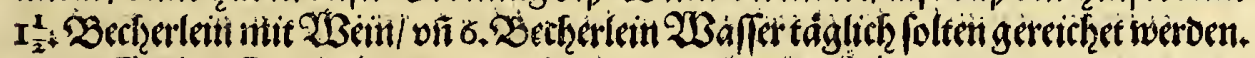

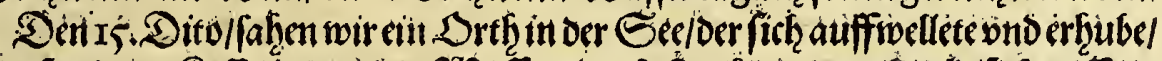

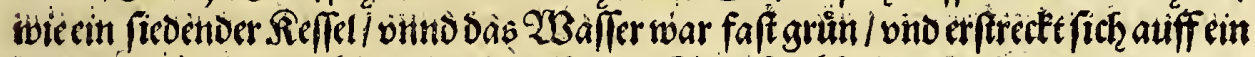

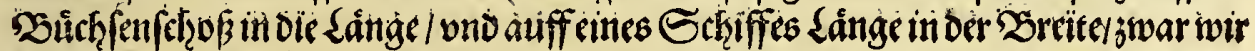

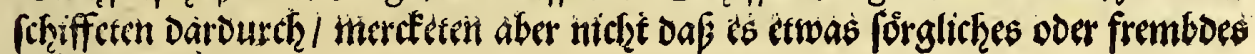
wother gerwert.

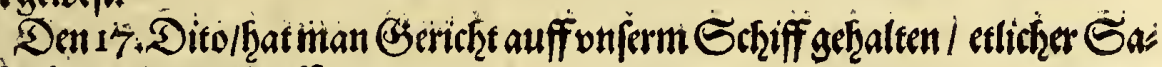

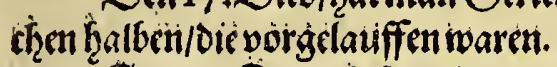

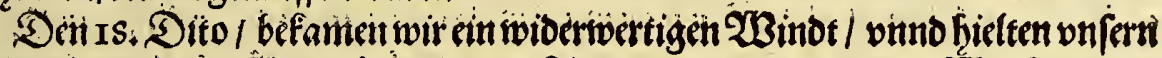

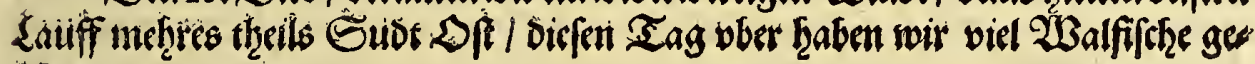
ferticit.

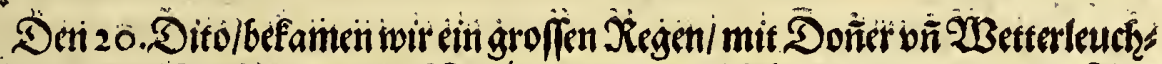

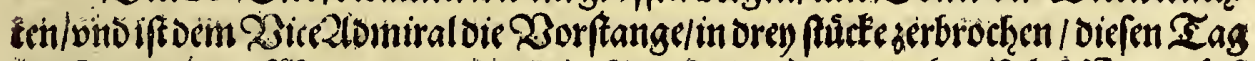

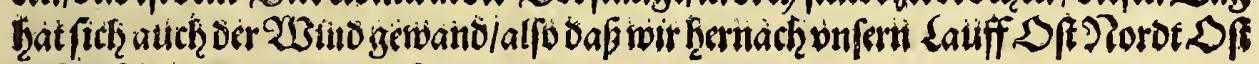

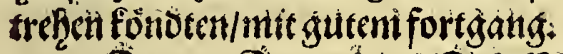

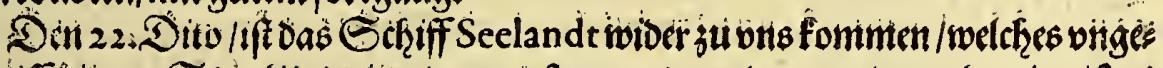

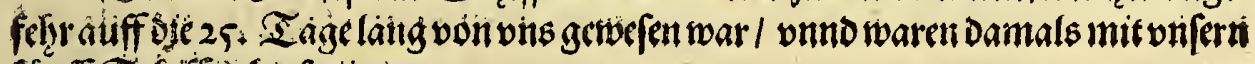
fünf Echiffict beyfanintal.

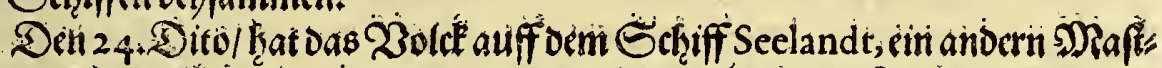

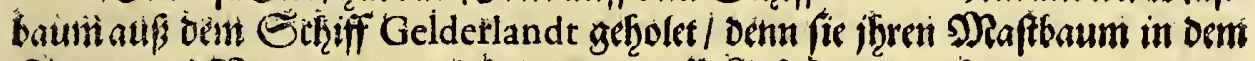

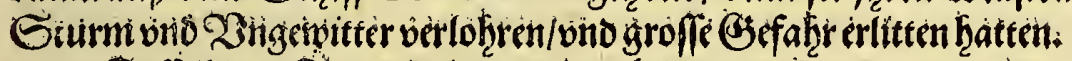

Deffelbigen Sages heaben wir bas Lainde bon S.Laurents; oder Madagafcaf

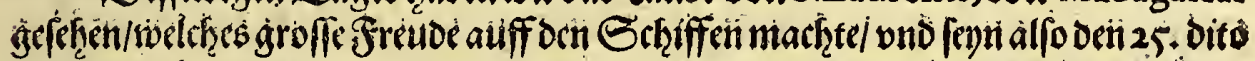

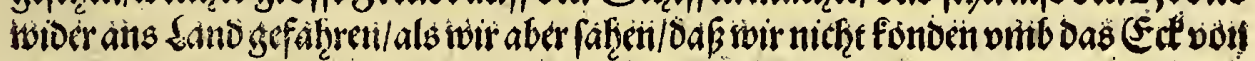

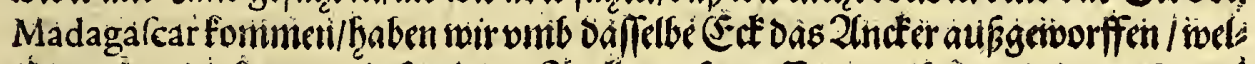

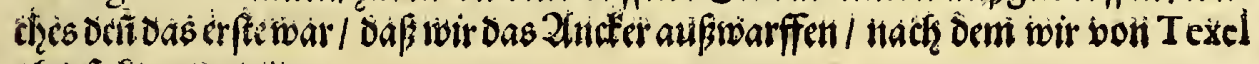
abgefofircn itationi:

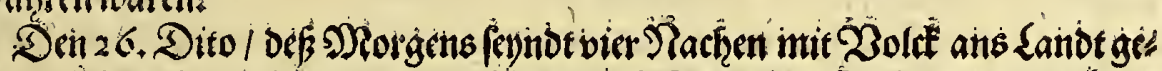

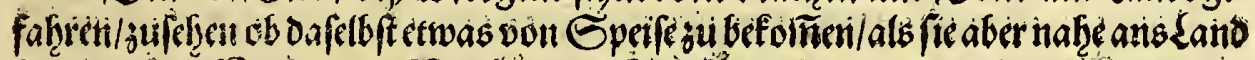

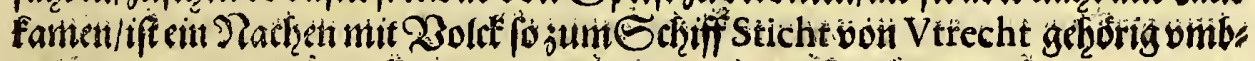

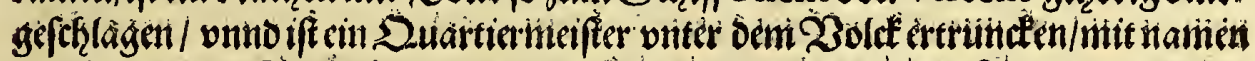

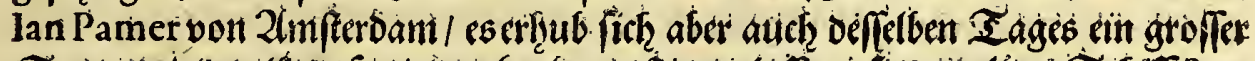

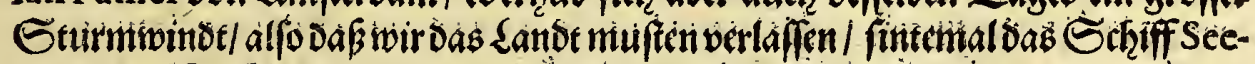

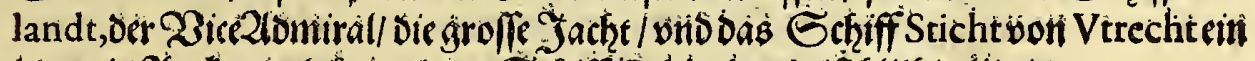

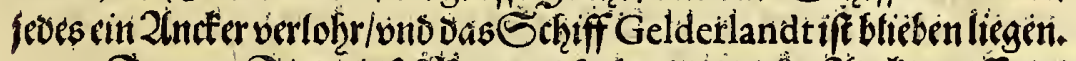

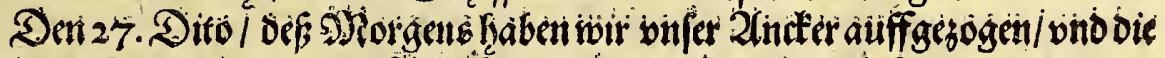
Eegel gerichetet / Den atiderti Eekiffeti nach juvolgen / Da befaimen wir guten

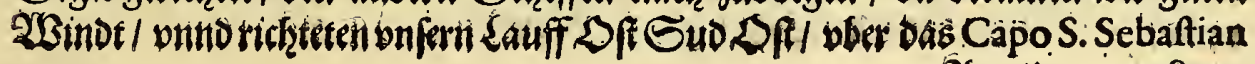
It ij oufonts Detrit ogits Tohoett. 


\section{Drientalifęen Şitoien.}

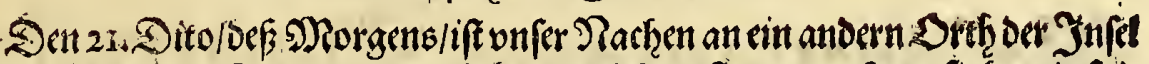

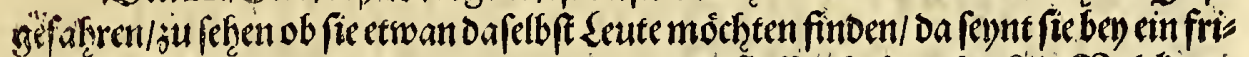

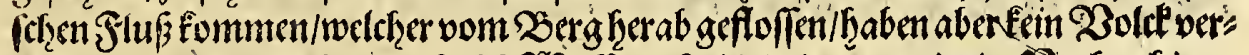

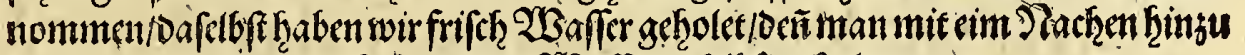
fafgren fondec/vio war faft bequem 2 b affer bafelbft zufiolen.

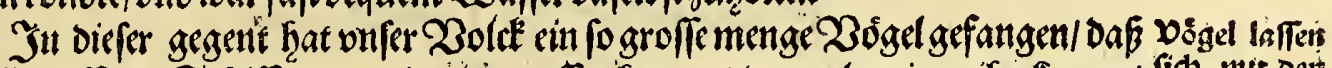

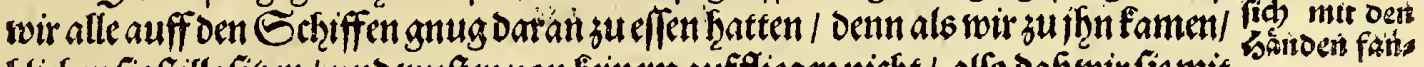

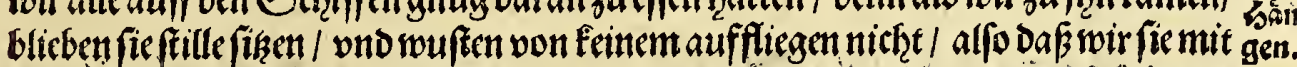

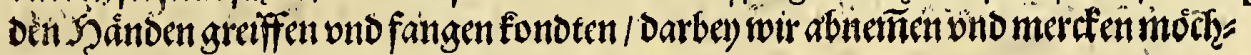
ten/Dafesc cin onberwohnet Sande feyn mufte.

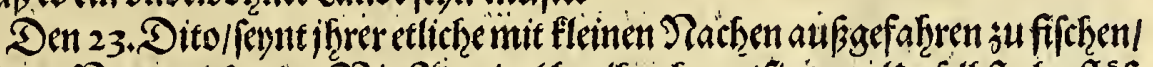

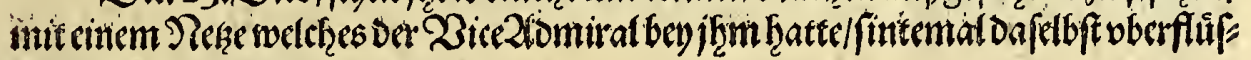
figviel Fifche verfiandern waren.

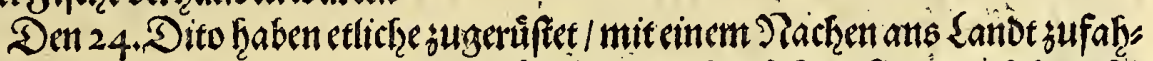

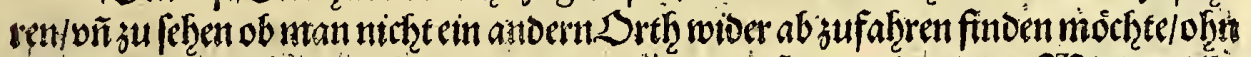
allein Den / Durch soclchen wir anfommen saren'/ fintemal onts Der WBinde nicht Dienen wolte/ Denfelben 2 Beg roiber jubrauchen.

Den 25. Dito/peynt efliche von oenen fo autsgefand twaten/ndiber formen/fias

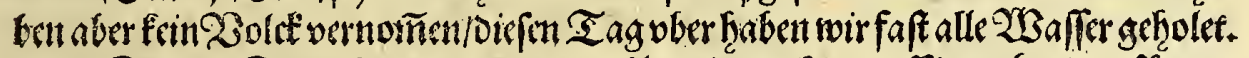

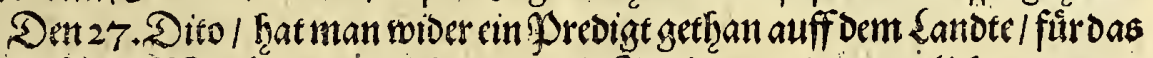

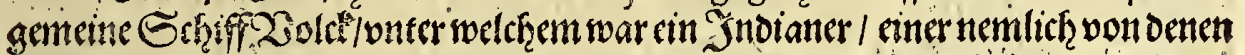

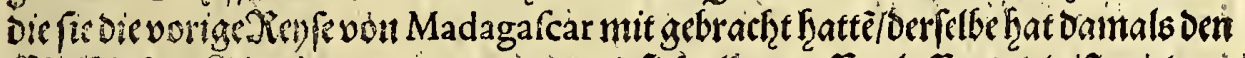

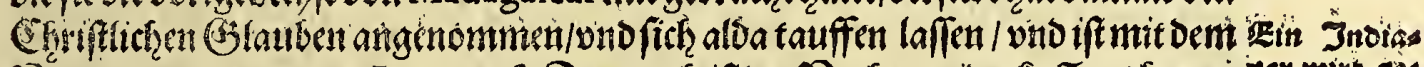

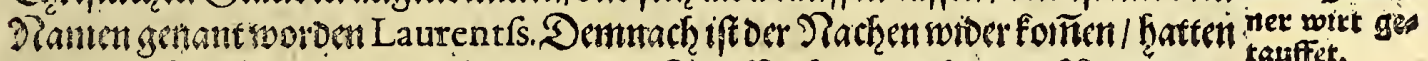

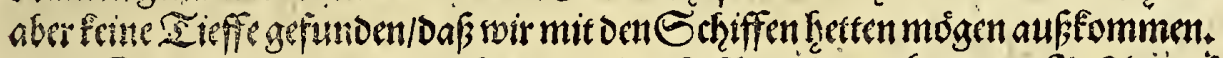

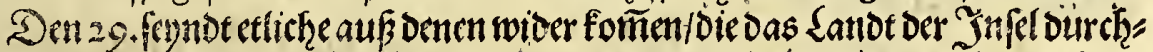

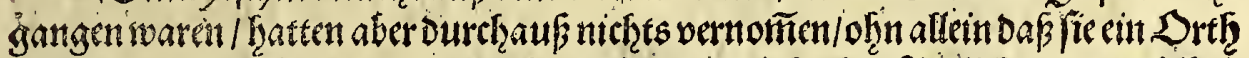

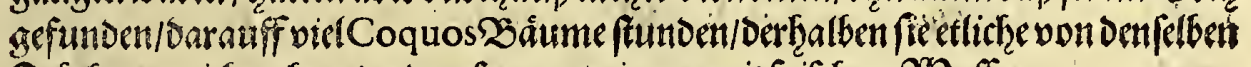

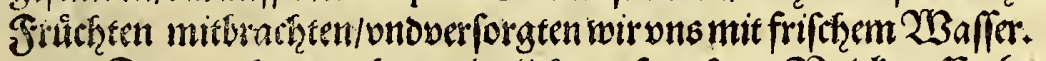

Den 30. Feynot abormal etliche aus onferm Bolde auffe Sande gelauffon

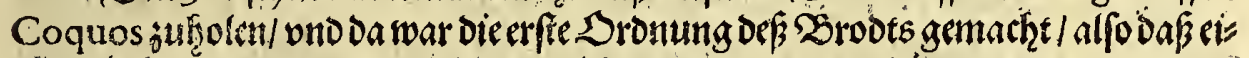

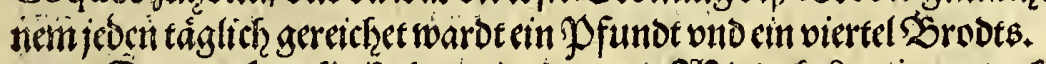

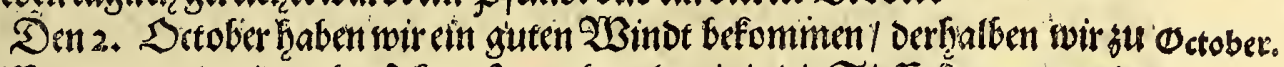
Eefieff gangen wno wiber abgefahren fenn/aber als wir in bie Tieffe famen/ waroe os gank fitlle / alfo das wir die Sichiffe Durch Shilffe def Ruvers in Den Rachen

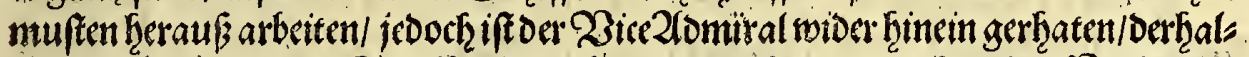

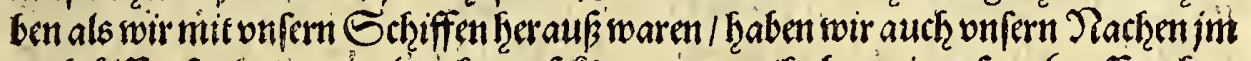

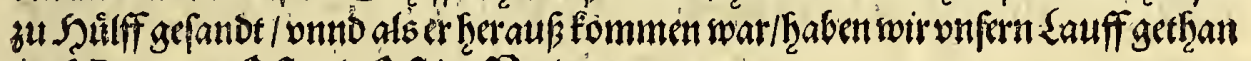
nach Bantam; Oft vito S St ins Norden.

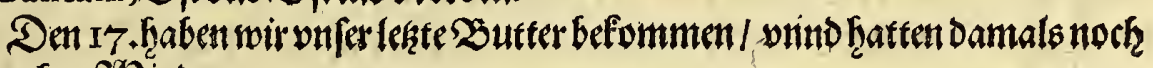
in rautfen 2 indt.

Den 2s,vnd 29. Dito / wardas WBafer dép Dieersfaft weif geferbet Daher

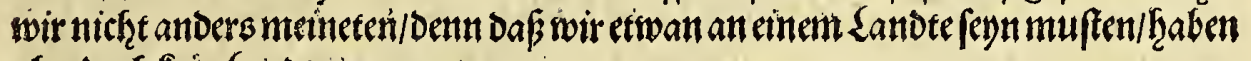
aber Doch fein Satot bernommen.

Deffelben Tages haben wirdic Gonne umb den Mittag vber bem Shaupt die Gonne

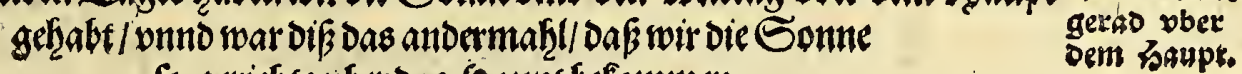
fo gerictics pber Das Saupt befommen batten.

2 iij $\mathfrak{B e f f r t e t}$ 


\section{Drientalifecten Snoien.}

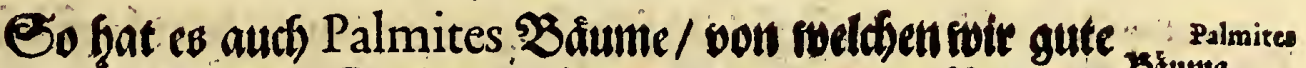
Erfriffyung befamen. Diefe souct) fen soie die Coquos $\mathfrak{B}$ ăume/

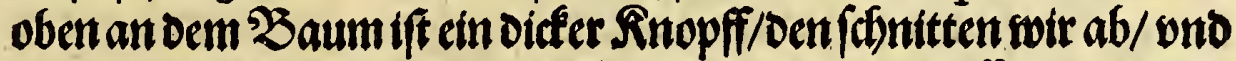

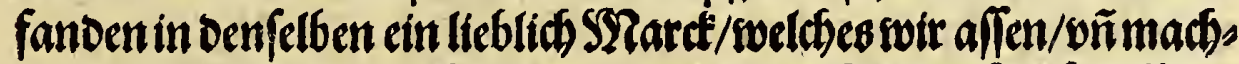
ten audf bifsweilen ein Sallat barwon / soelches vne faft febr Purs gierte ond Erfrifódete.

WBir fuffen mit Dem meiften theil de 3 Boldfs ans Sant tono

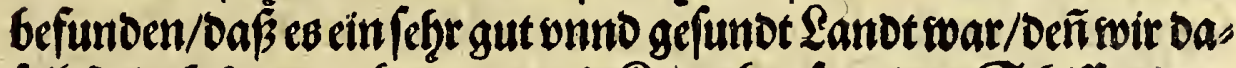

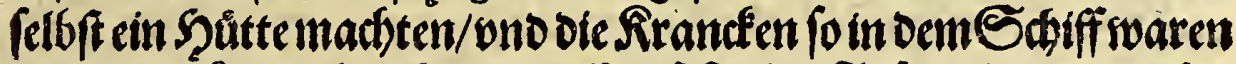

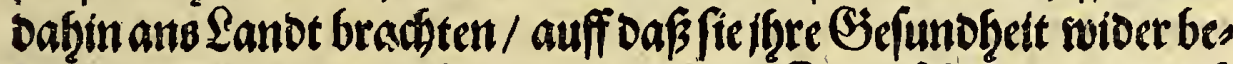

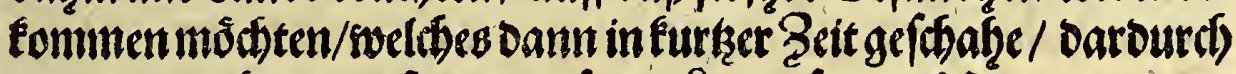

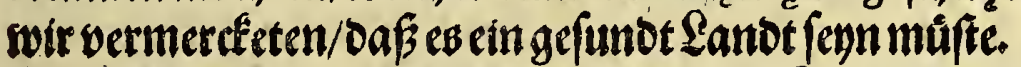

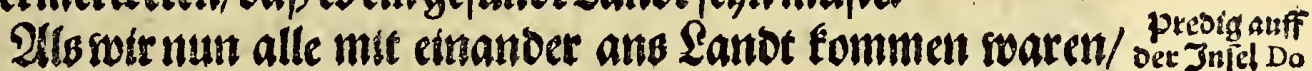
hat man vor vno nach mittage etnPredigt gethan/onnoGott Dem ${ }^{\text {Cerne. }}$

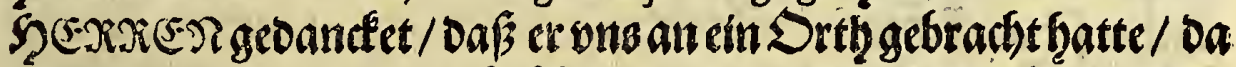

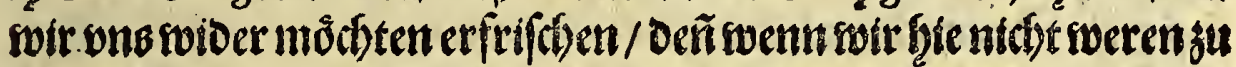

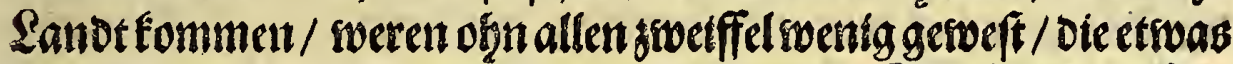

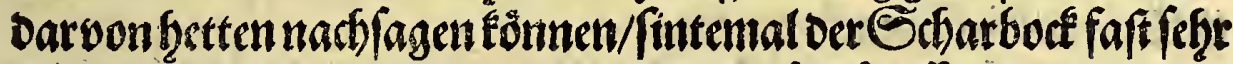
begunte vberfinat sutemmen / vinto onfer 23 affer megeentheils

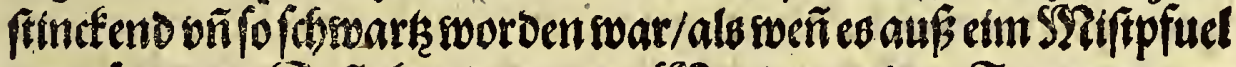

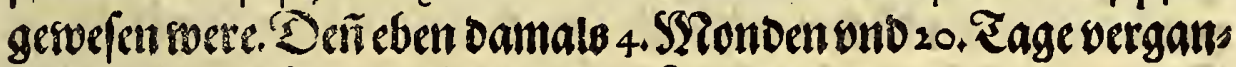

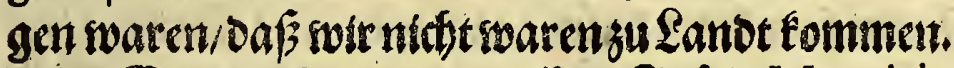

Demnacis soir nun auff Der Infeln Mauritius swaren/ife eln

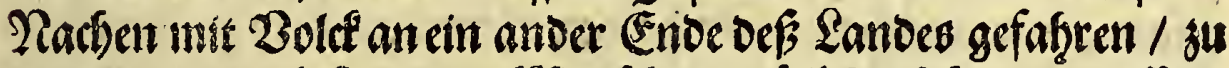

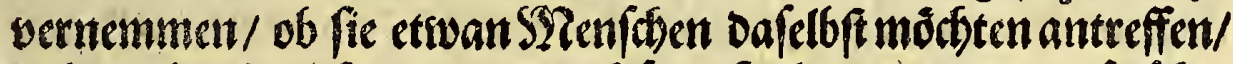
baben aber feine funden / feood) fenn fie fommen an ein frifobes

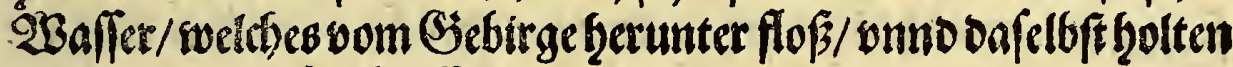
wir bernats onfer $23 a f f e r$.

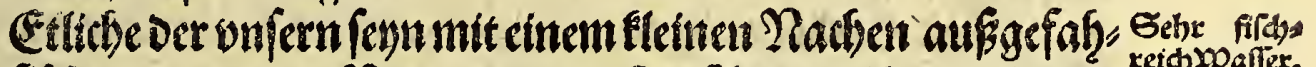

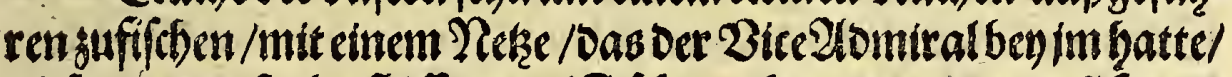

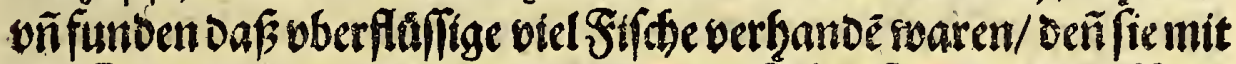

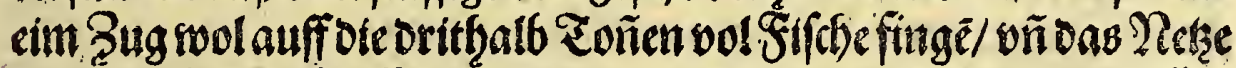

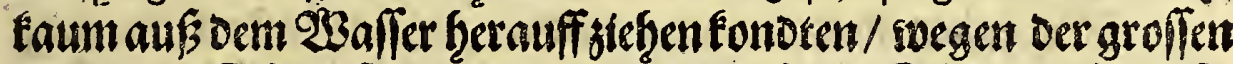

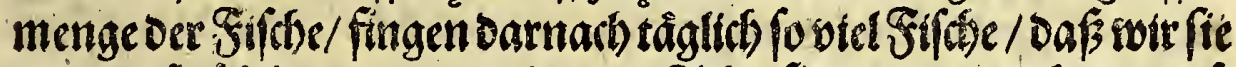

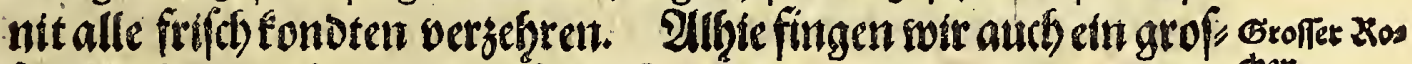

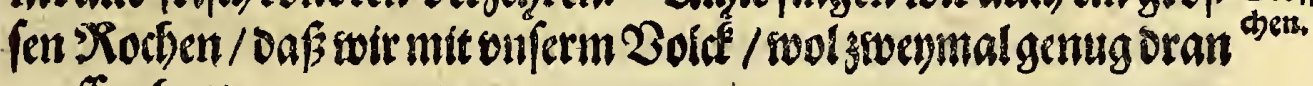
zueflenfaften.

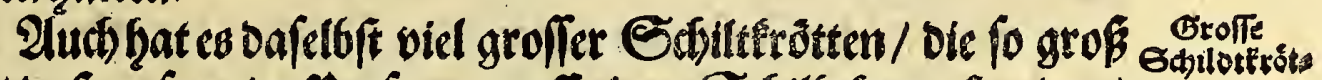

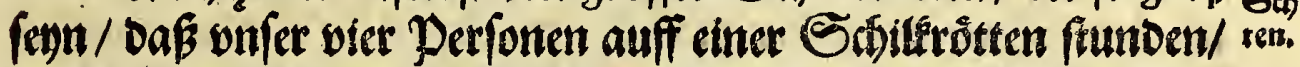

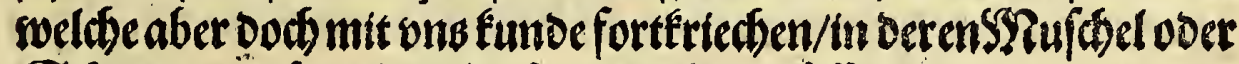
Edfielpen/onjersehen Perfonenhabengefefen.

G5?melte 


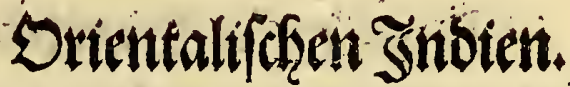

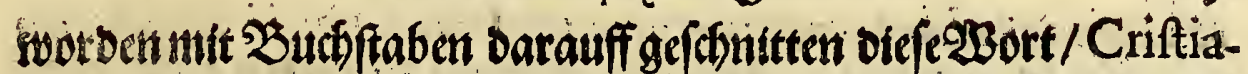
nos Reformatos, Giereformirte Sftriften/onno bas Wapen von Şolland/Seclanot ond 2lin/terban.

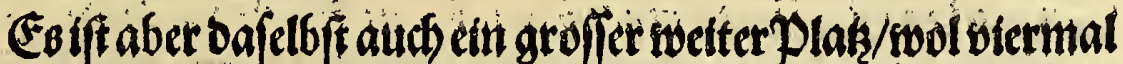

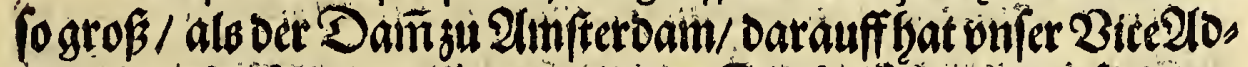

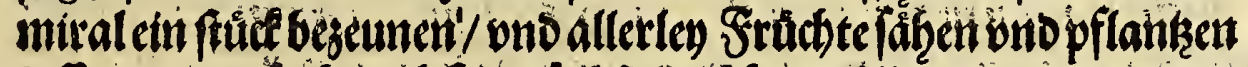

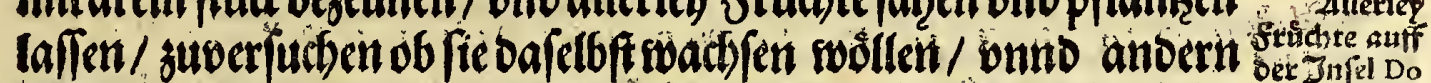

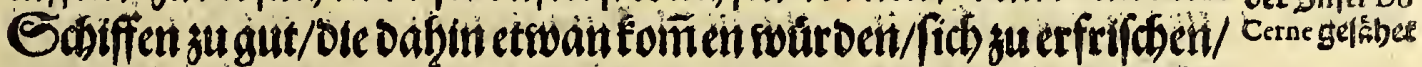

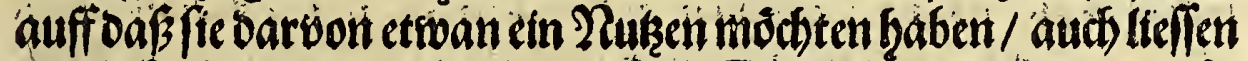

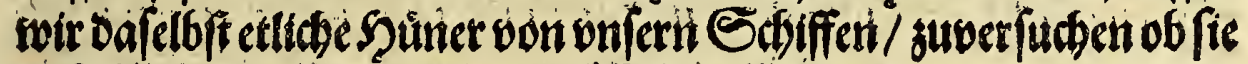

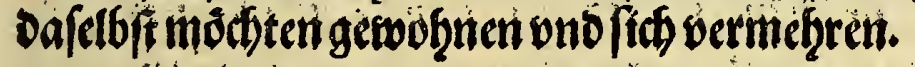

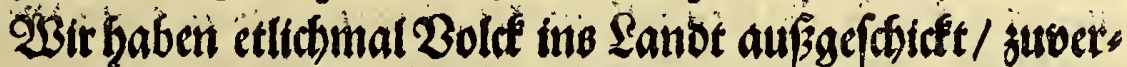

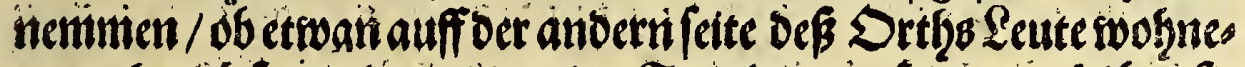

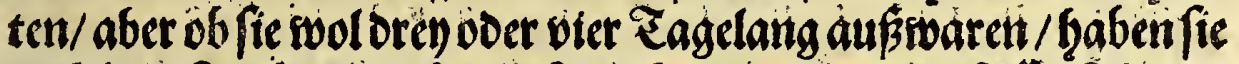

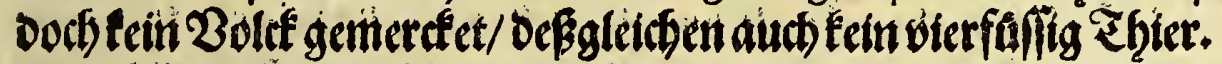

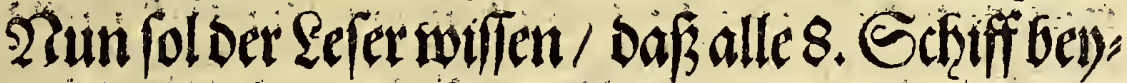

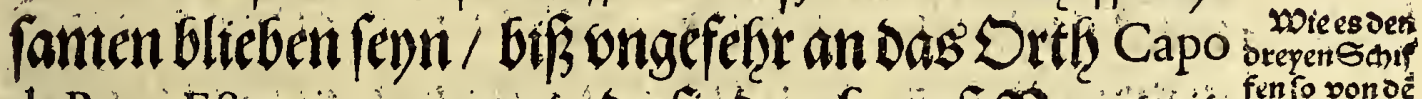
de Bona Efperanca gênant / oa fie ourch grofz Yngetoit ter oon ectionder gethetlet worben / oens. Sugufti

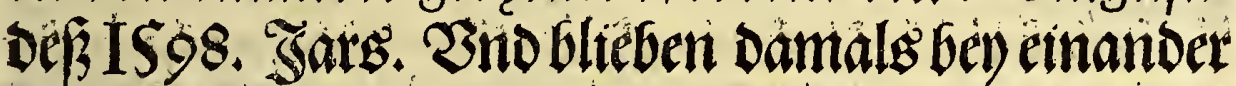
bielefunff Sofiffe/ nemblich oas Schiff Amiterdam; Seelandt, Gelderlandt, Vtrecht, wnino bas groffe sägld fiff Vrieflandtgenant/ oiele worben oertrieben auff ote snlel Mauritius, wnins lagen dafelb/t I4. Tageftlle/

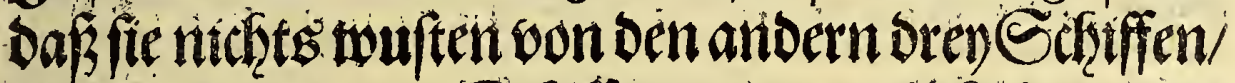

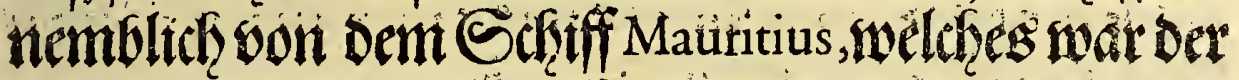

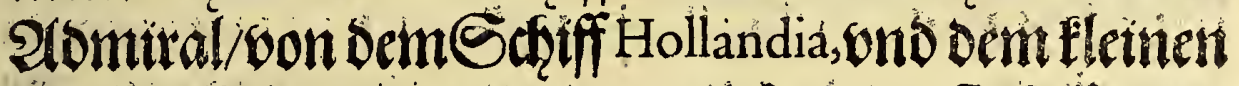
șagferff Oueryel genant / welche ored Echtfentt einander an die Sinfel S.Mària gelanded/ono voti ban.

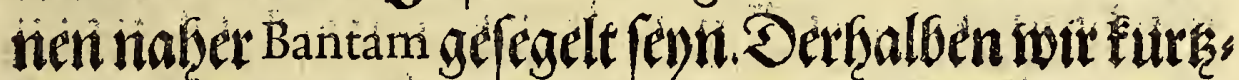

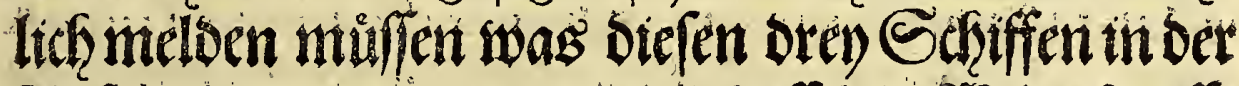

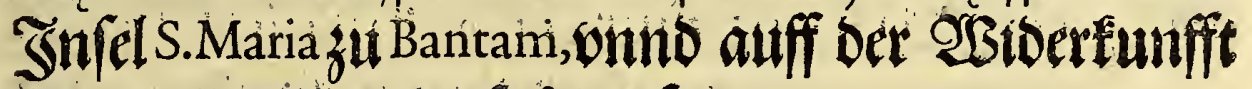
begegnet/wono siberfatien /eb.

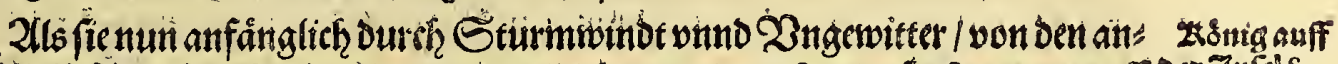
Dern fünff Eethiffen abgefondert vitnd vertriebentworden / feyndt fie fommen auff der Znjels.

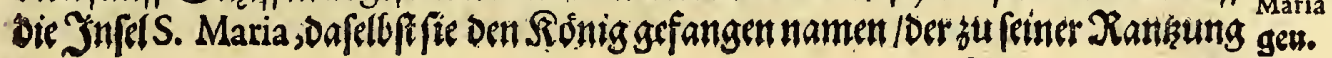




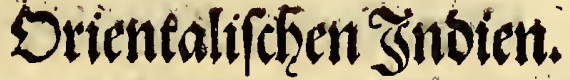

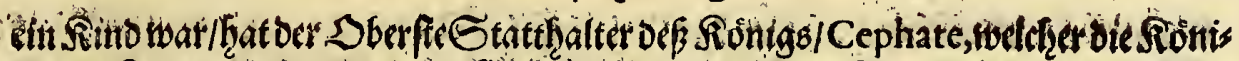

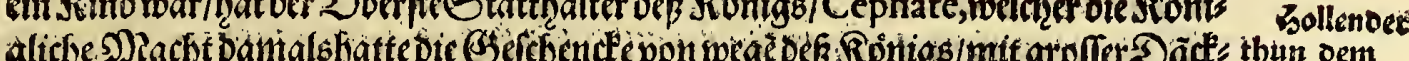

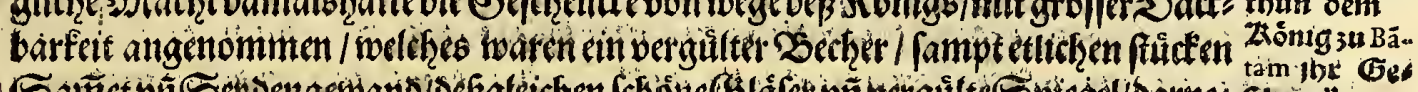

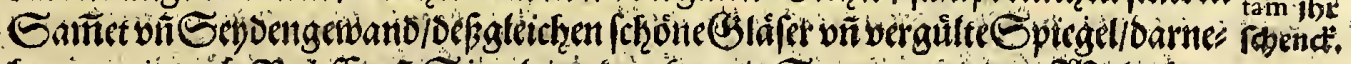

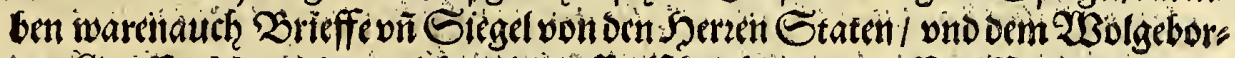
nen Goraffen Mautitio, swelche nitit grofer Eefrerbietunglauff Der Eroefrichendel cmpfä̀ngen vond àtgenomtnen wor bent.

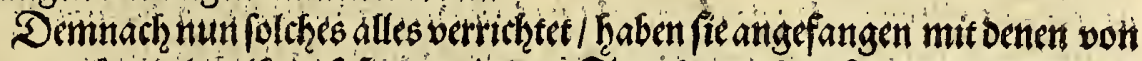

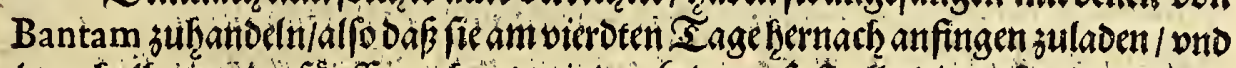

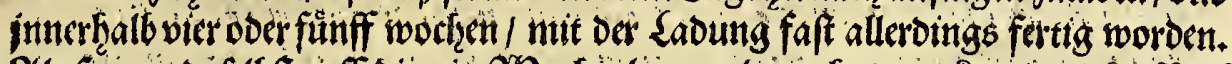

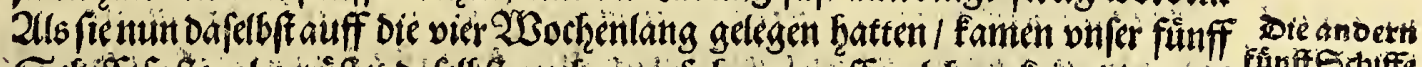

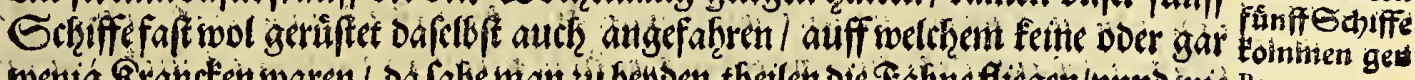

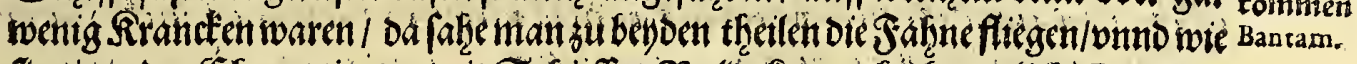

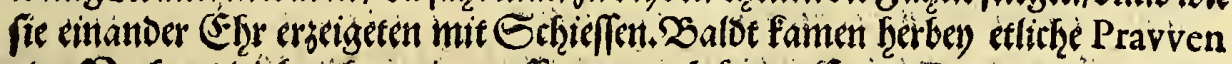
nder Machen / biebrachten ein grofle menge Shiner / Ever / Coquos, Bonanas,

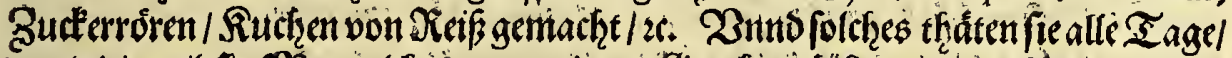

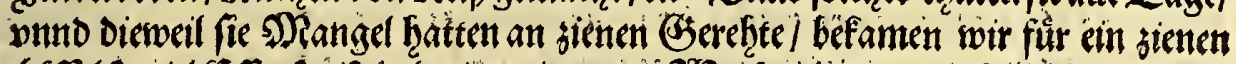

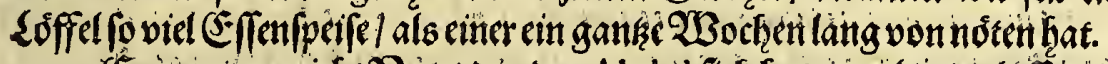

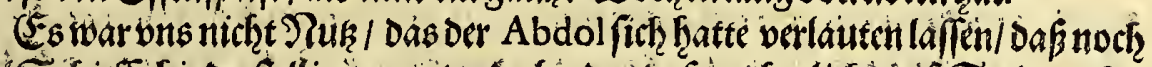

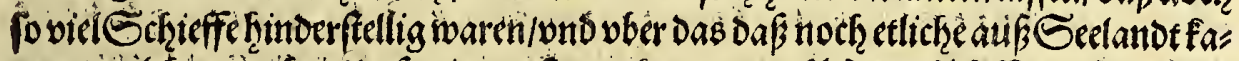

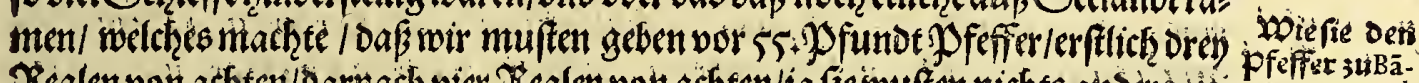

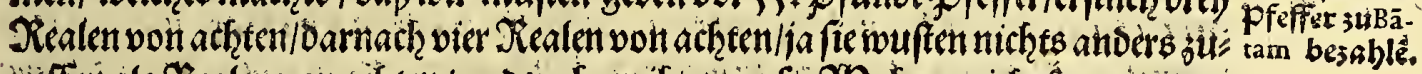

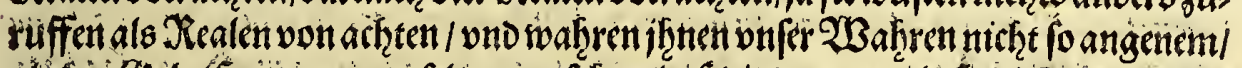

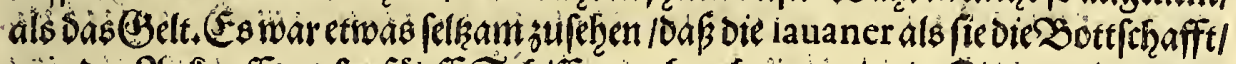

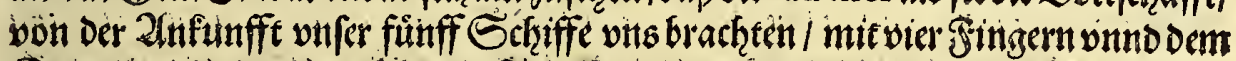

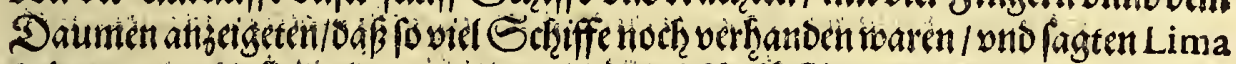

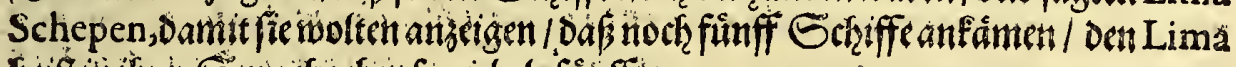

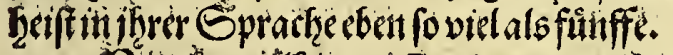

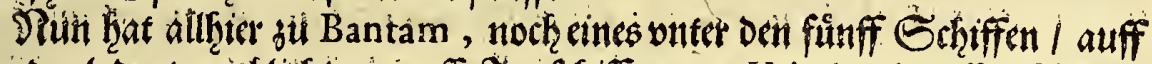
Solanto geladen/ nemblich Das groffe Yagfchiff genant Vrieslandt,Deffen Echiff: mann war lacob Corneliss, vinno Werwalter Wouter Willekens. 2lis fie nun

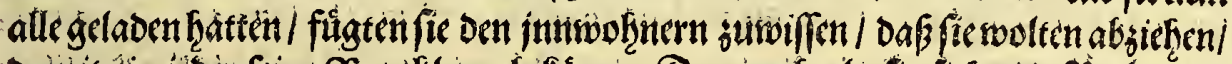

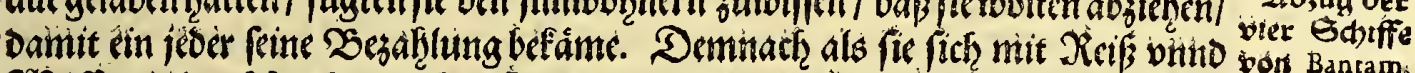

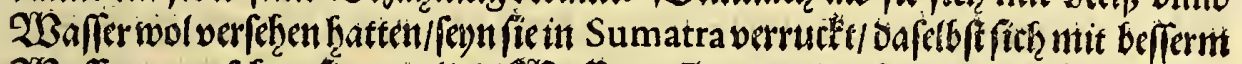

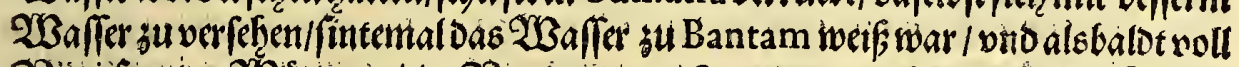

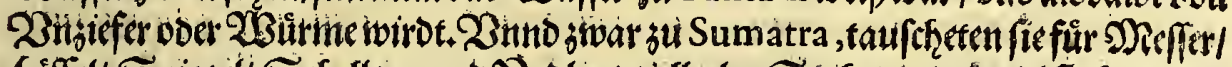

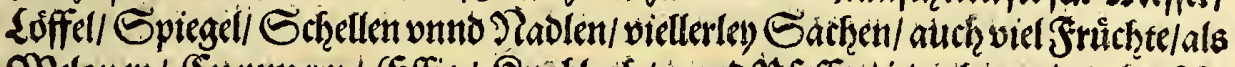

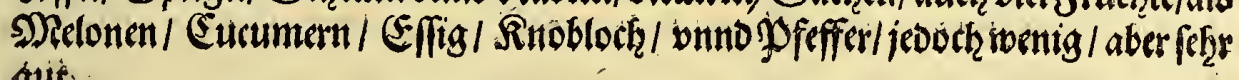
gutit.

Efliche ₹agevor

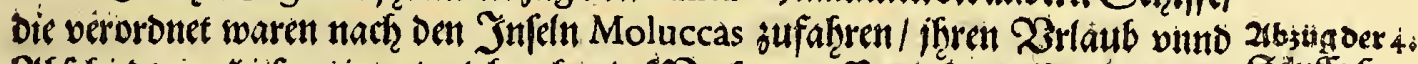

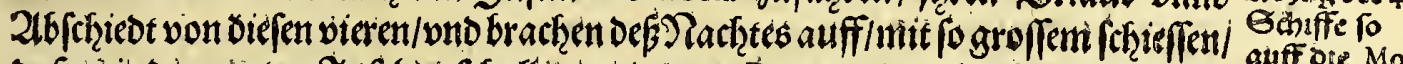

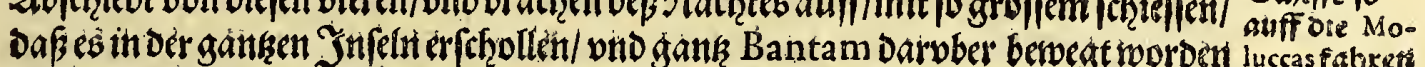

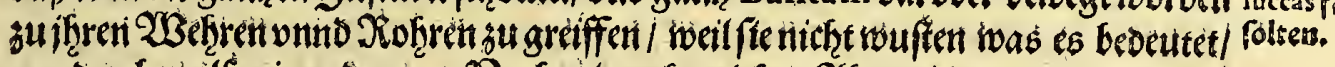

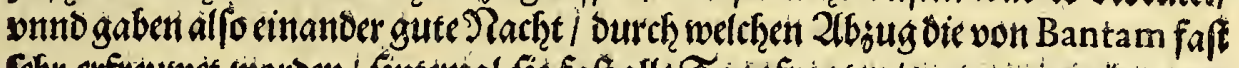

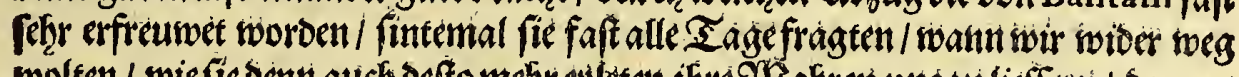

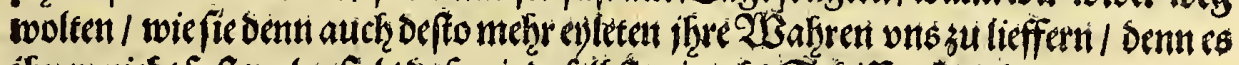

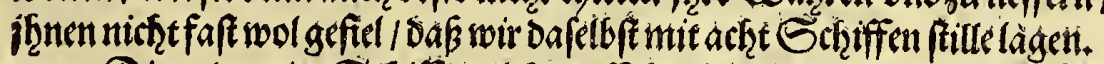

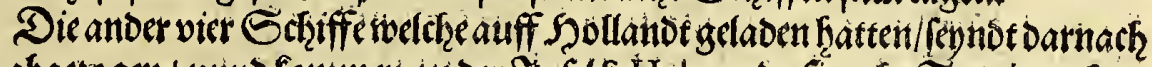

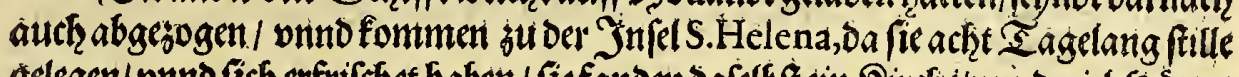

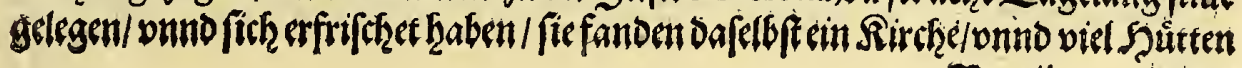

B ij in Der 
Orientaliferen \$noien.

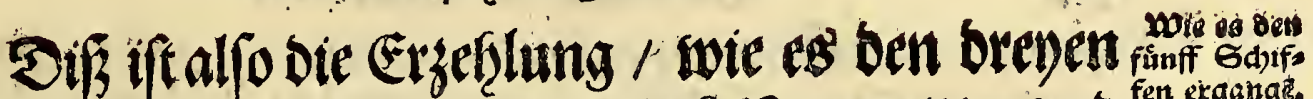

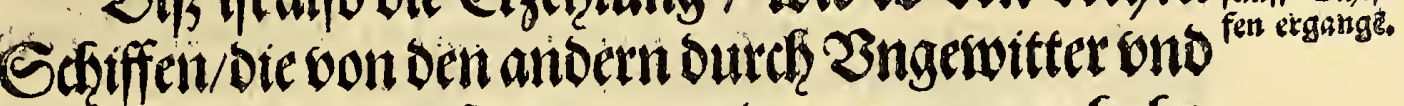
Sturmivind abgefondert worden/ ergangen faabe. Fun iwollen wor in ber $\mathfrak{h}$ if tori forffafteren onnd bes

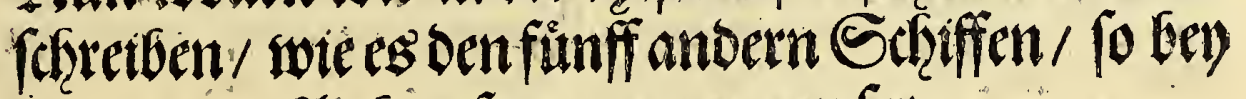
cinander geblieben/ferner ergangen $(\mathfrak{e n}$.

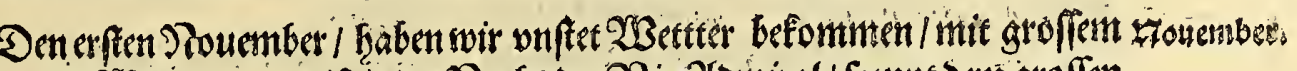

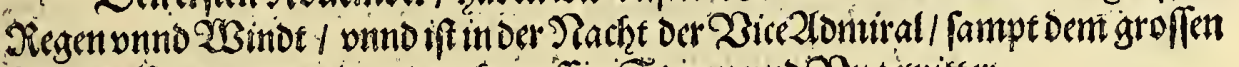

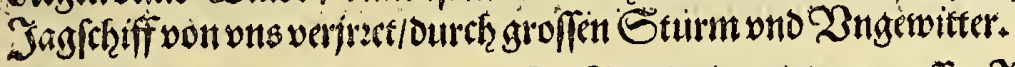

Den oritten Difoliftenfer Wireldomiral / mit orm groffen Jagfcriffiviber.

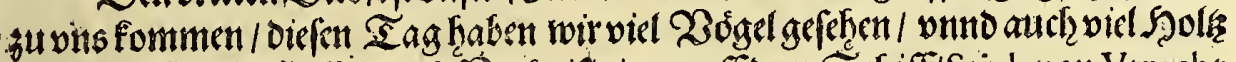

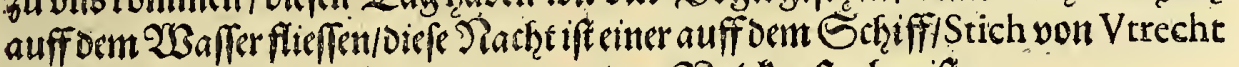
Geftorben/welcher der erfferwar/Der vnter bem 3 olde geftorben ift.

Den 6. Dito/ift nach Sittage ein 2Birbetwint an vnfer Grthiff fontment

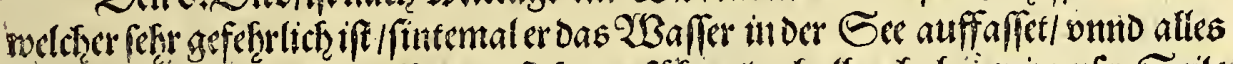

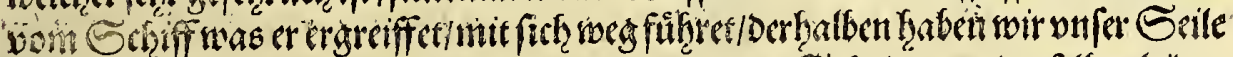

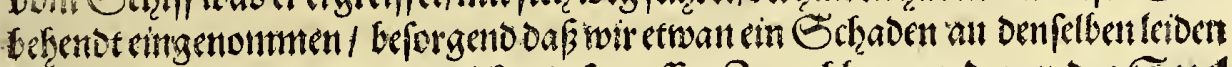

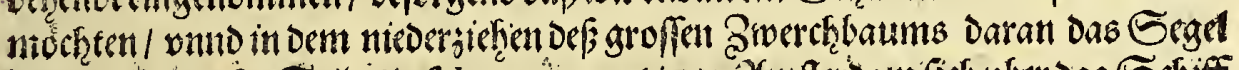

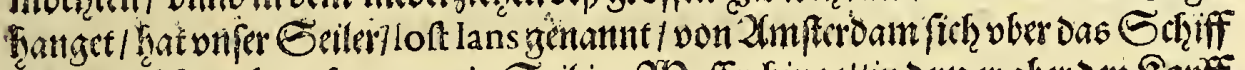

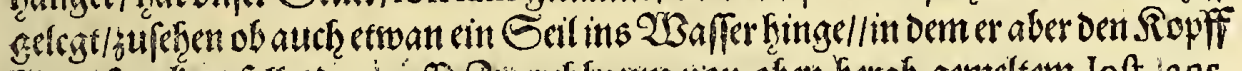
Ginatis rectet / fellet ocr grofle Bwerch baum won oben hetab gentelem loft lans,

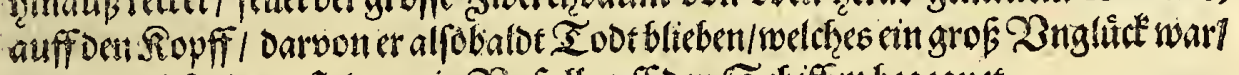

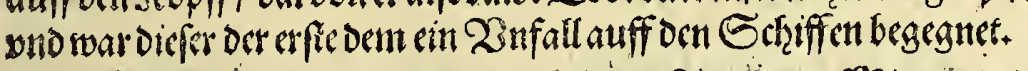

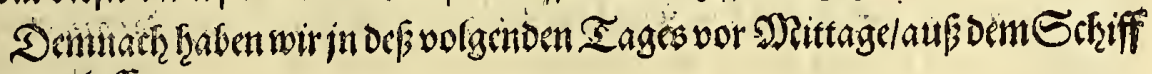
younnsgrlafict.

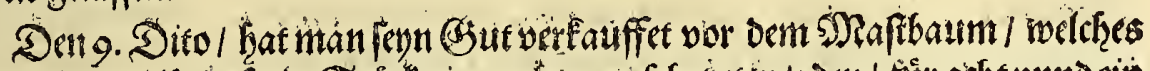

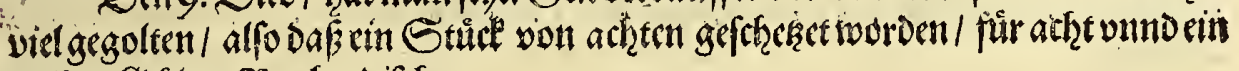
,halben अutten Sorabendifh.

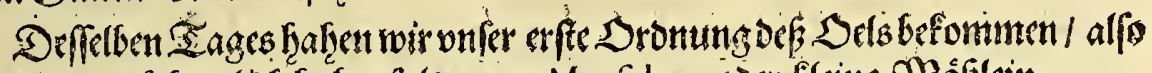

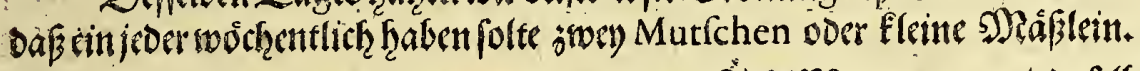

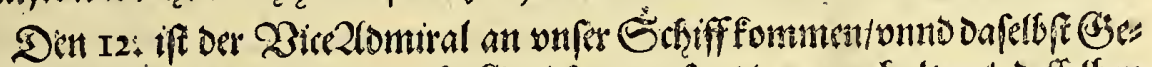

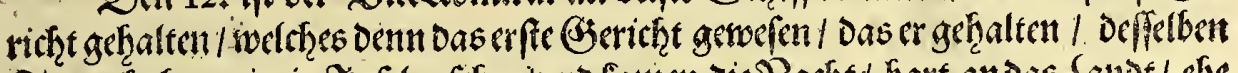

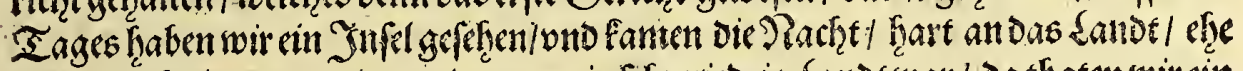

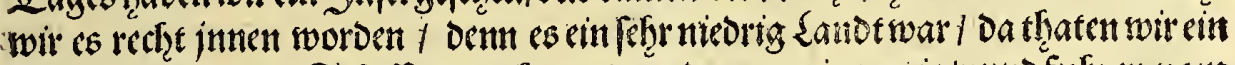

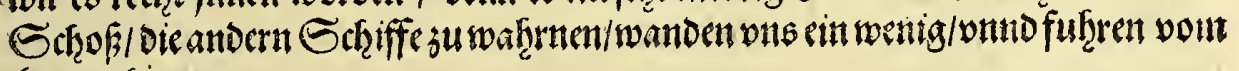
Sandabe.

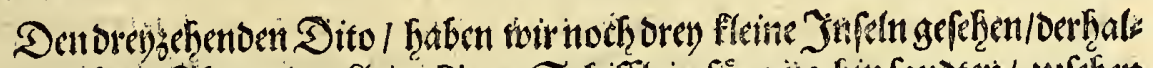

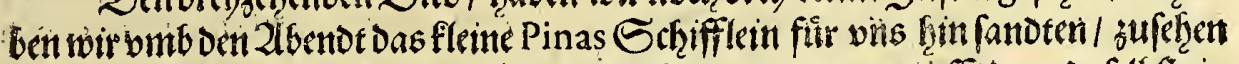

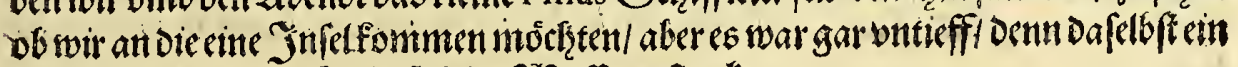

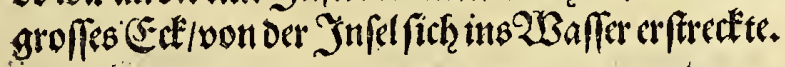

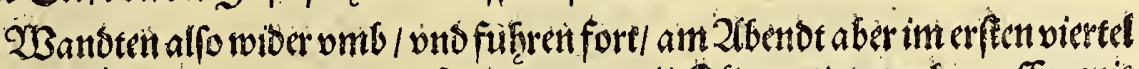

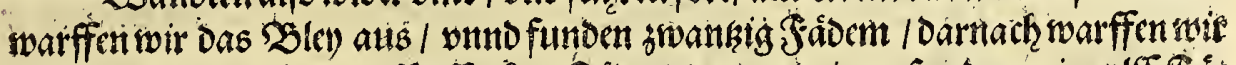

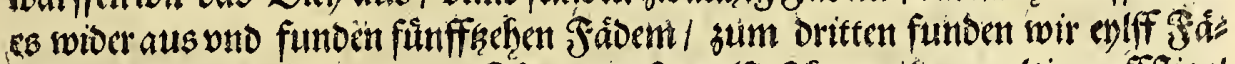

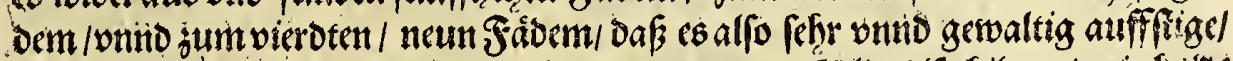

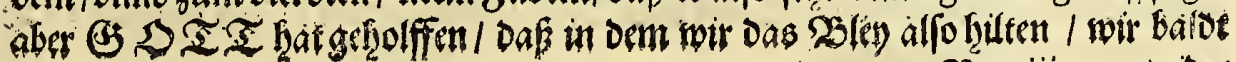

$\mathfrak{S}$ iij witore 


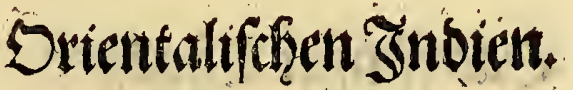

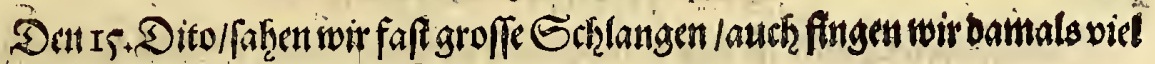
Sifth Bonytes genatiot. finfonote.

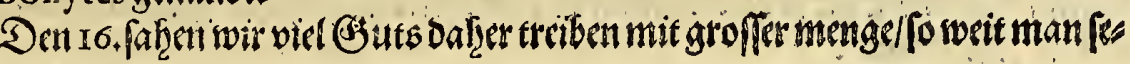

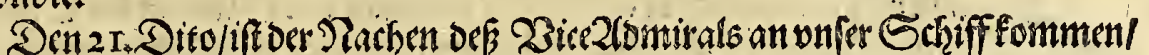

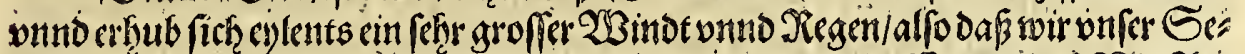

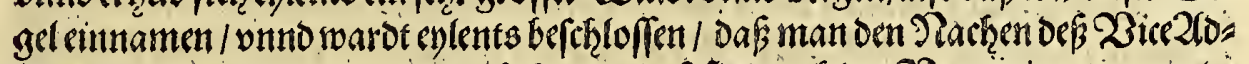

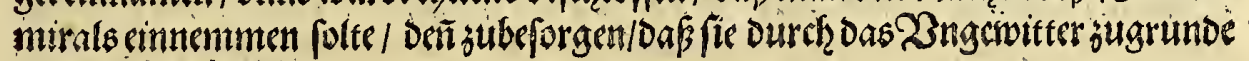
fietten getien mivgen.

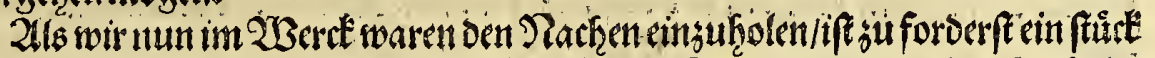

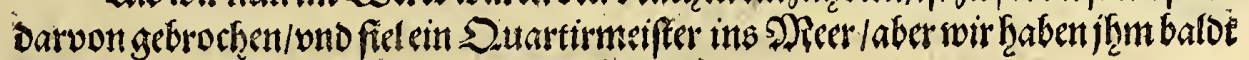

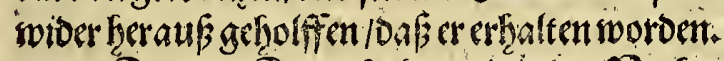

Den 25. Dito / haben wir in Der Nacht vengefthe bres Gtunden vor tagel

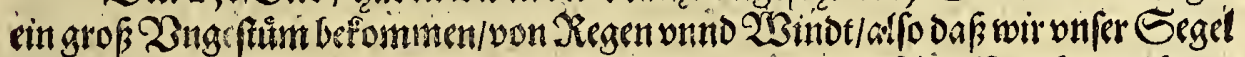

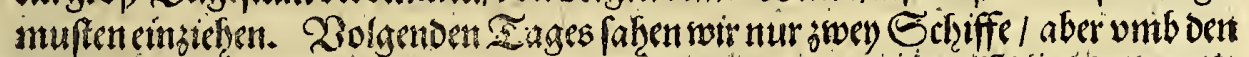

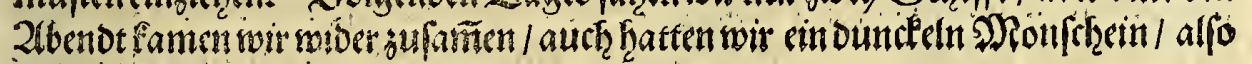

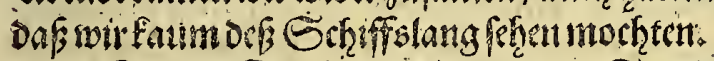

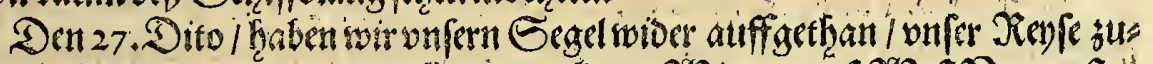
forbun / von hatem Damals cin fenen truckenen WBind / auß $2 B$ ff Nortweftet

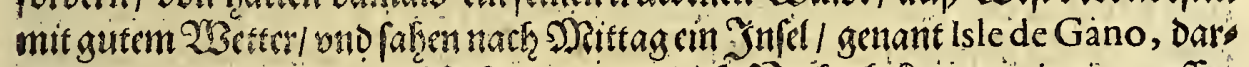

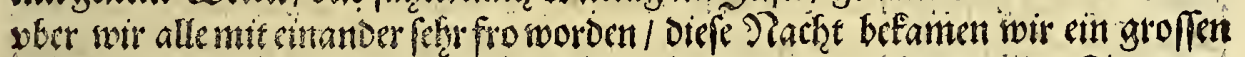

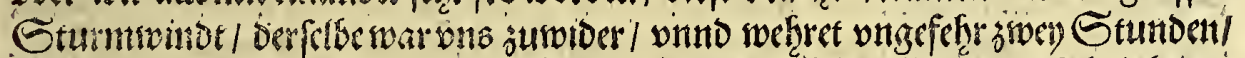

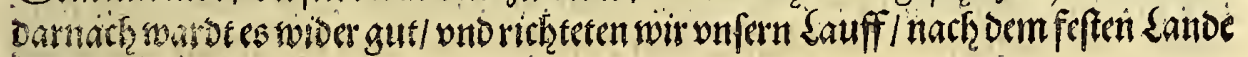
Sumatra.

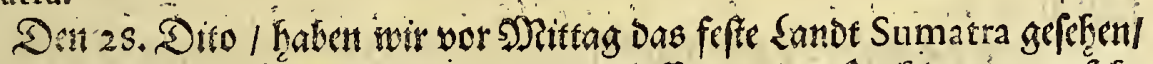

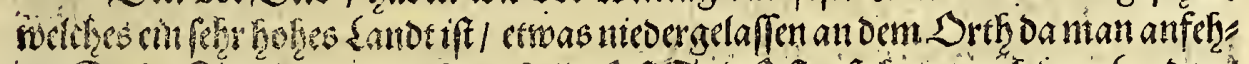

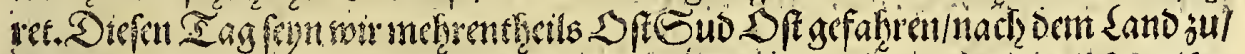

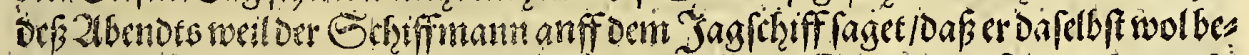

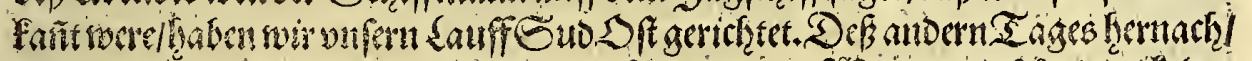

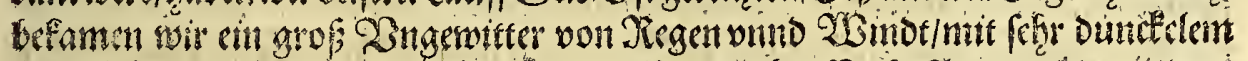

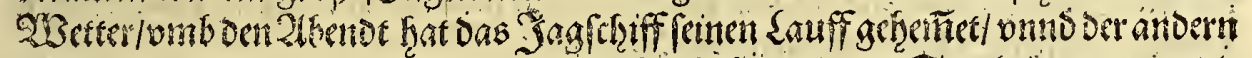

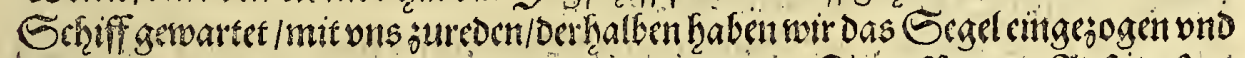

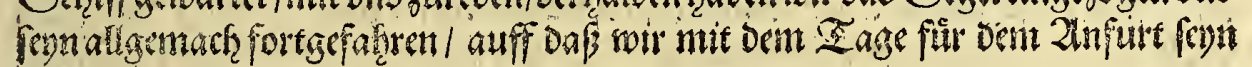
moithert.

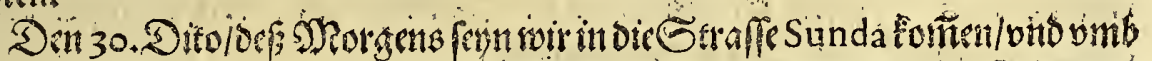

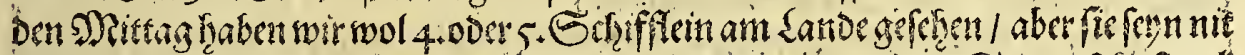

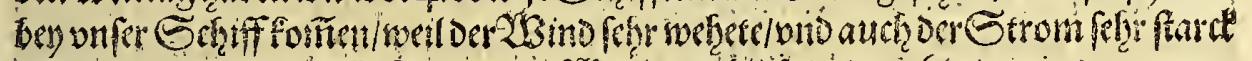

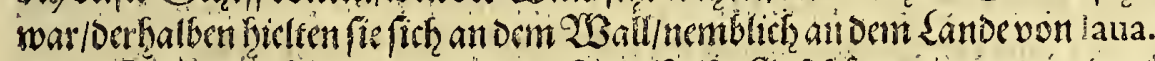

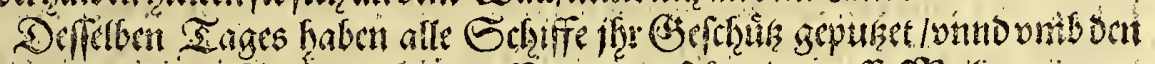

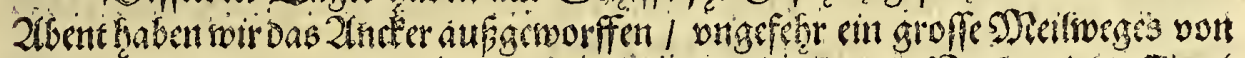

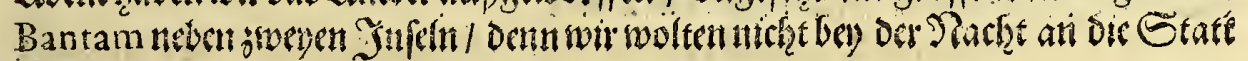
fafiren.

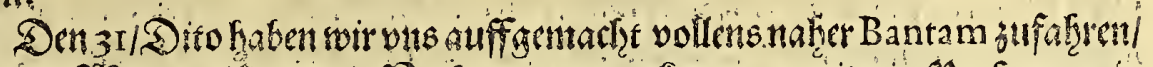

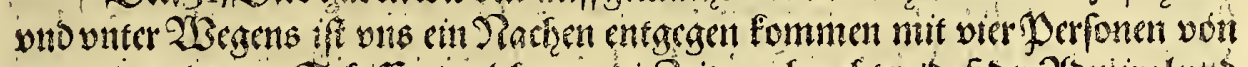

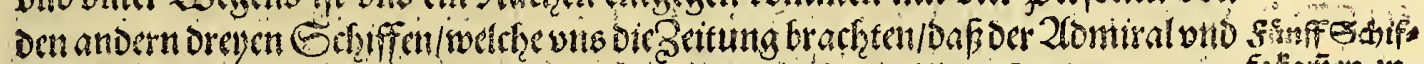

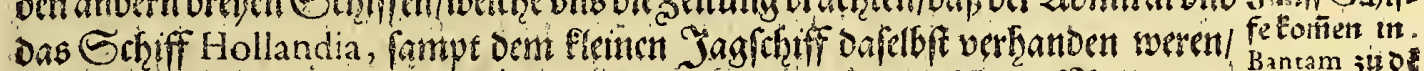

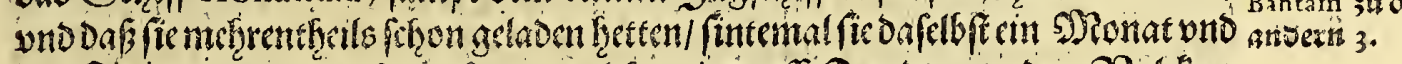

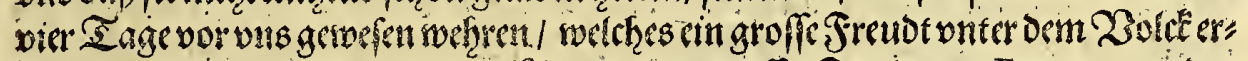

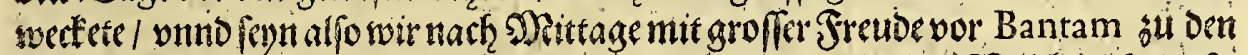

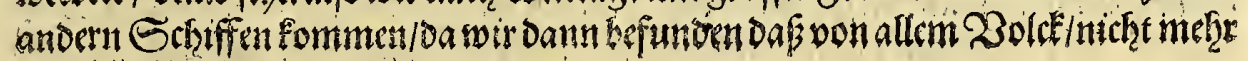
als nür is. Derfonengeforben waren.

פิeffelben 


\section{Drientalifęen Sinbien.}

Den 14, Oito/ Def Morgens ift cin leoiger Rachen ans Lanbe gefafiren/

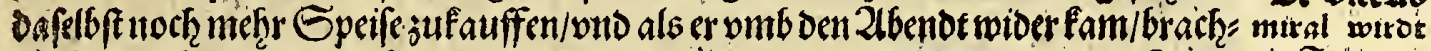

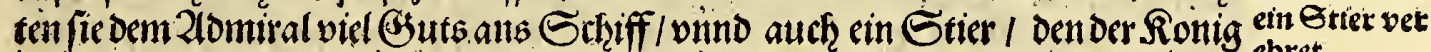

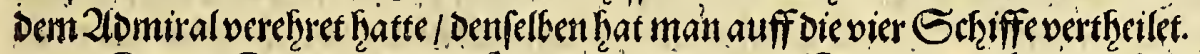

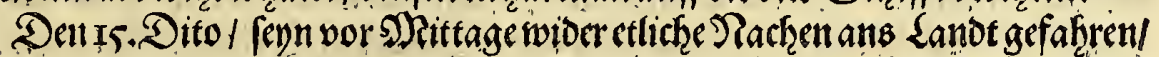

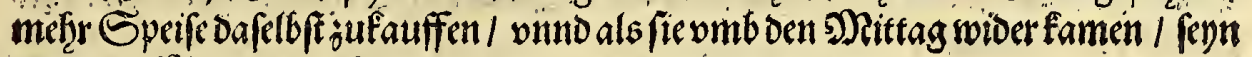
wir nach Me ittage auffigebrochen vnd won dannen gefahren.

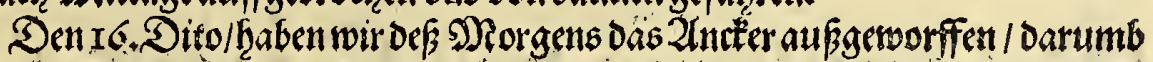

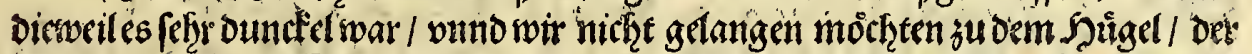

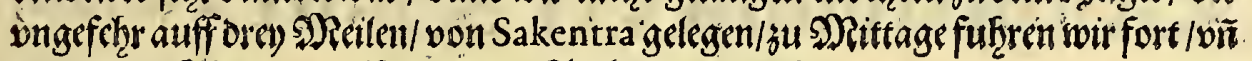

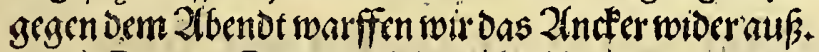

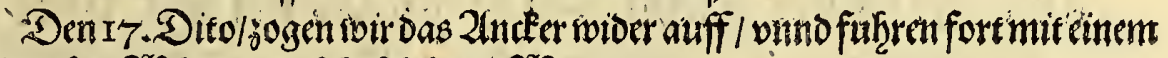
Rorowefen 2 Bind tond ferter fchóntem 2 Better.

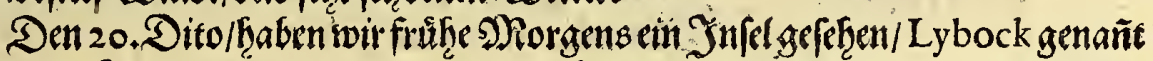

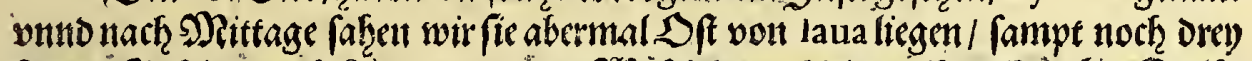

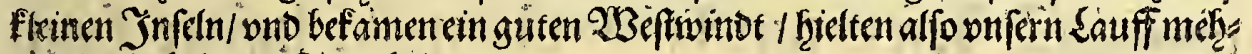
rentfieits Sftond SuD SPt.

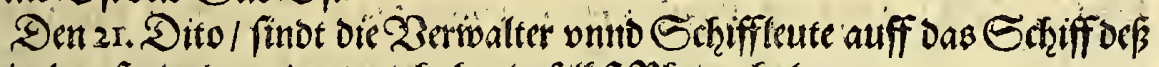

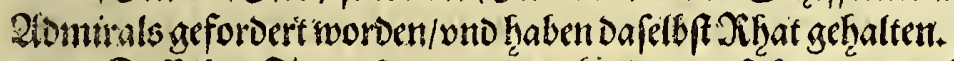

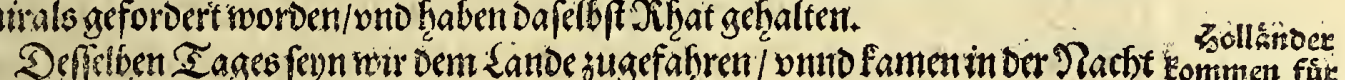

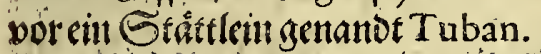

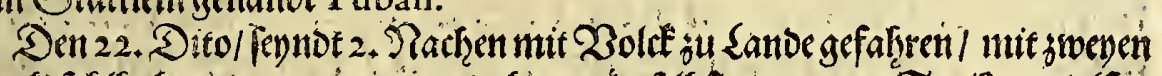

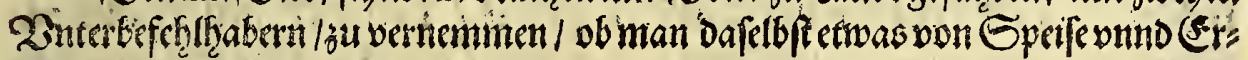
frifchung bePommen mơ chte.

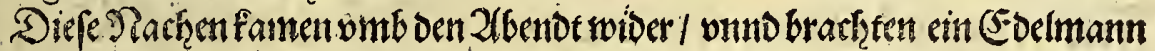

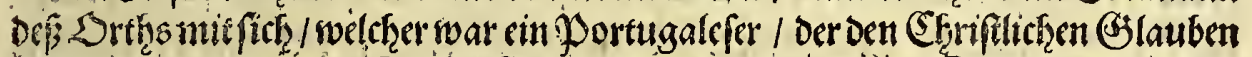

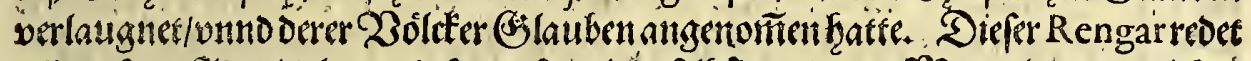

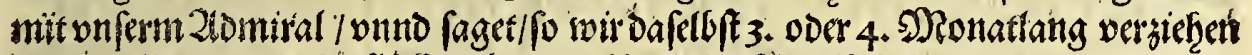

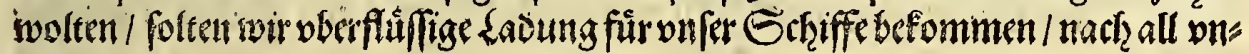
fermbegront.

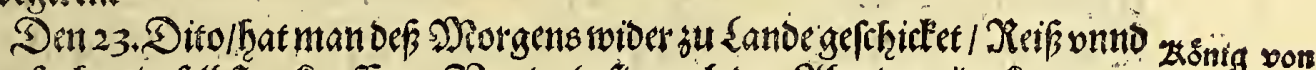
andere fachen Daferbit jufauffen. Wnnd alsfie vmb Den 2 bende wiberfommen/ Tuban ver-

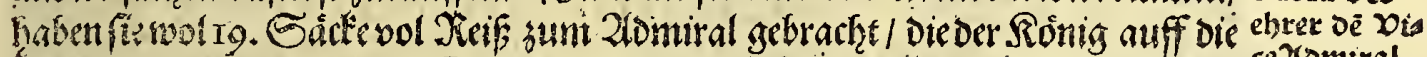

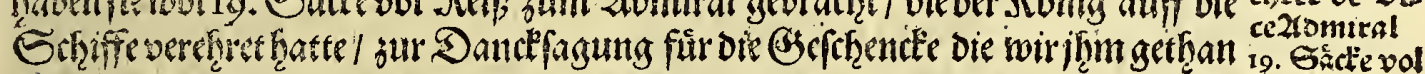

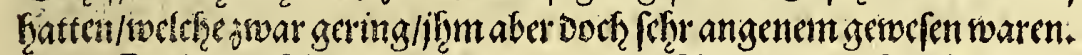

$$
\text { रieif. }
$$

Deffelben Iagrs haben wir vinb den Zlbend wiel 20 ofd's geferten auff allen

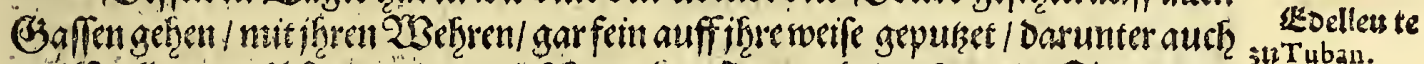

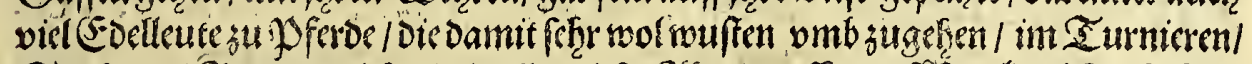

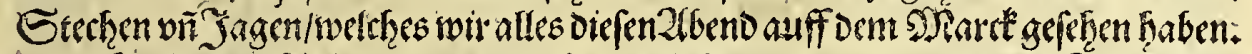

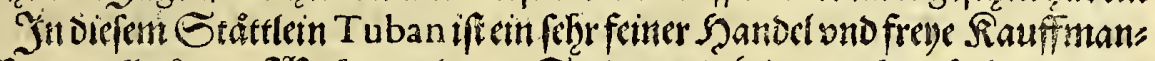

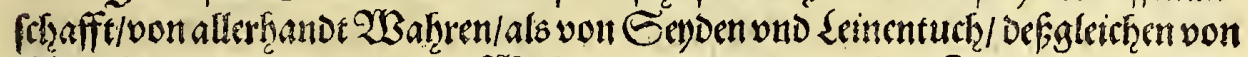

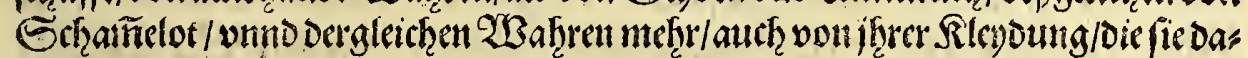

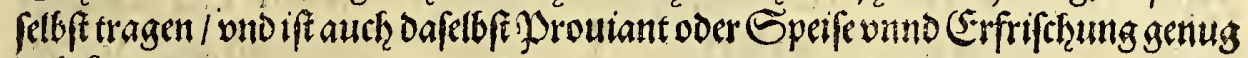
auberonmen.

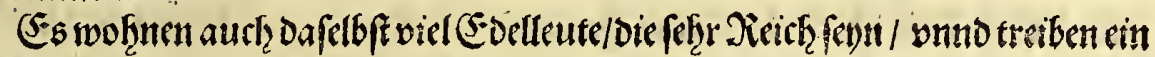

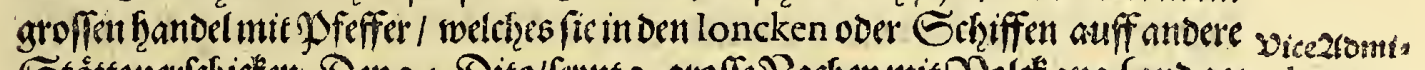

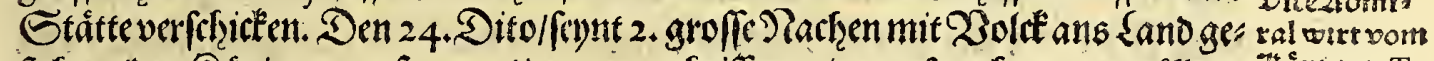

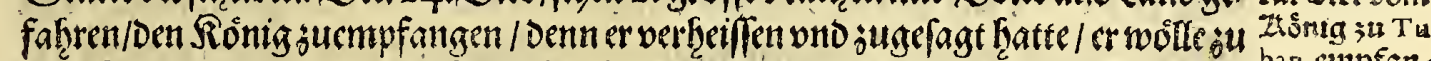

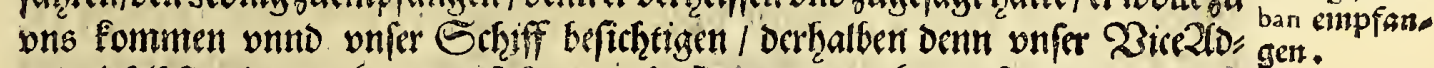
miral felbft mit ans sanot gefahiren / als fie nun ans sande famen / f̧at der

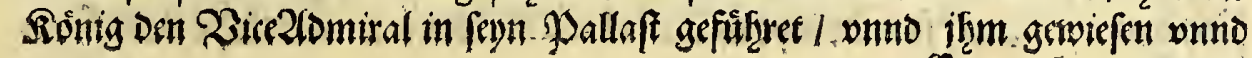

$$
\mathfrak{C}^{\mathfrak{g e j e} \text { iget }}
$$




\section{Orientalifferen Sndien.}

lidfem Syerfommen feun/ Die haben biel Diener vanto Selbetgene

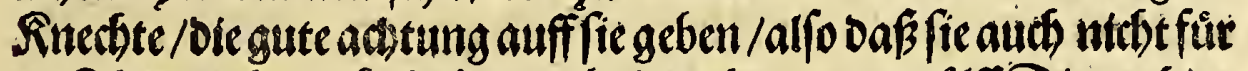

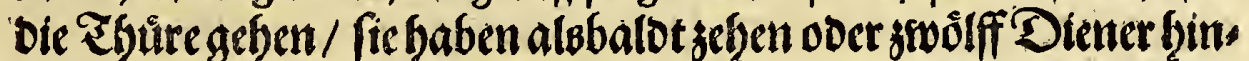

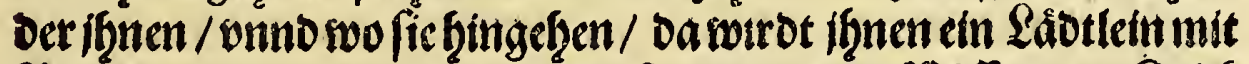
Betelblettern naibgetragen / bie fie mit grânen Rauffen onb Ralce

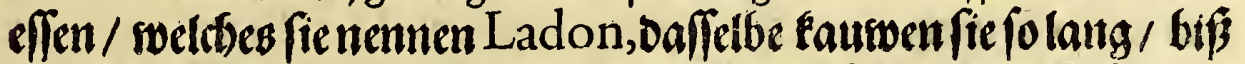

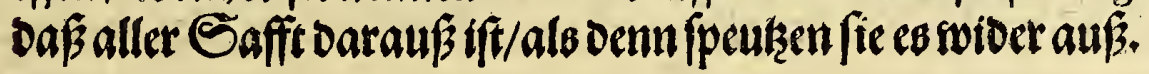

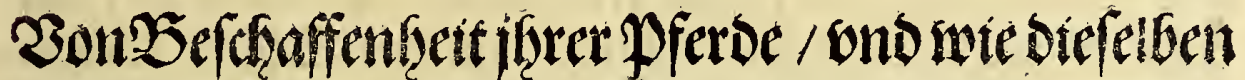

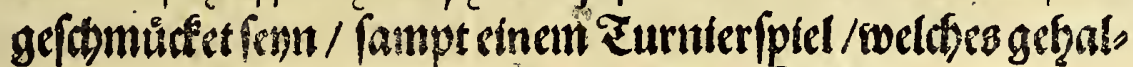
ten worocti ben 23. Januarij onfern Rauff leu=

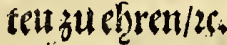

Gine Se Edelleute in Tuban, feaben einguten $\mathfrak{B e}$

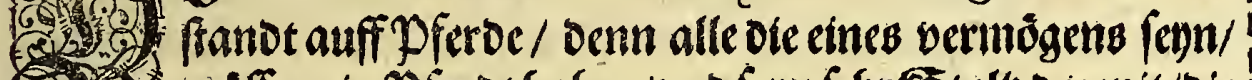

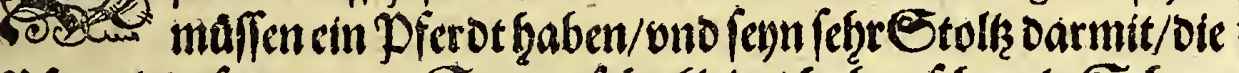

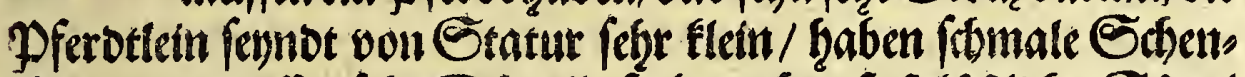
25erdseffins bett vno rís frung oer

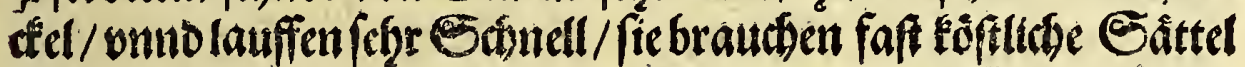
zu ifgren Dferoten/entweder von Sammet doer Epannififfem

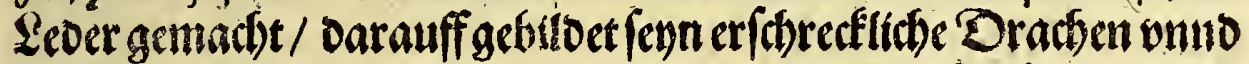
Teuffel/ soeiche vergutbet fenn. Eievergleichen fich faft mit wa"

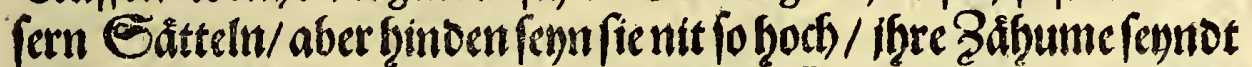

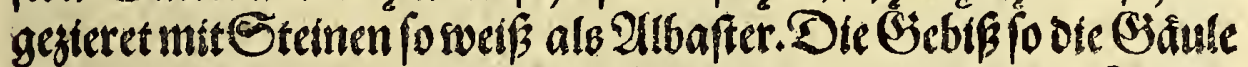

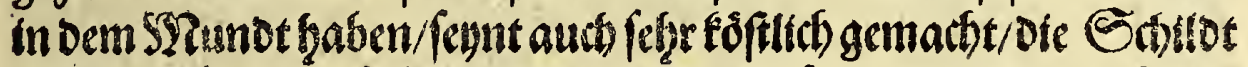

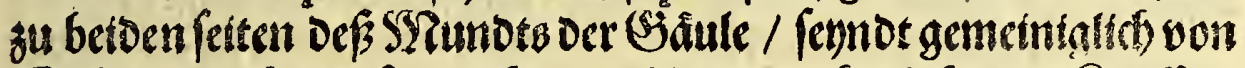

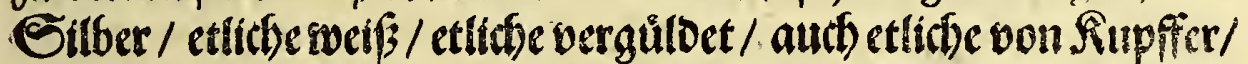

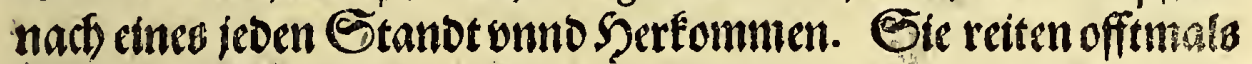

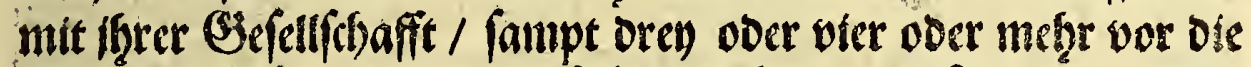
Statt hinaus / emander dafelbfit zu vben / Da fie Denn remen

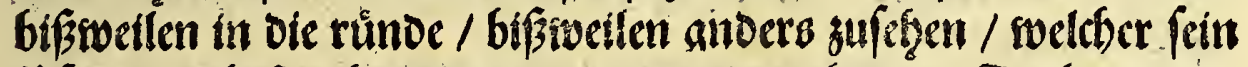
Pferd am beften befwingen vnno regieren fan / in ₹raben onno

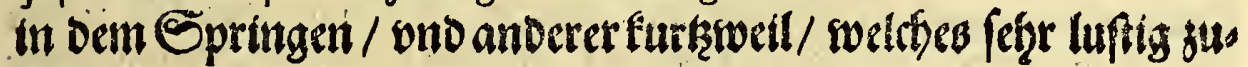
gehet.

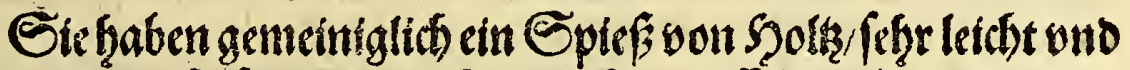

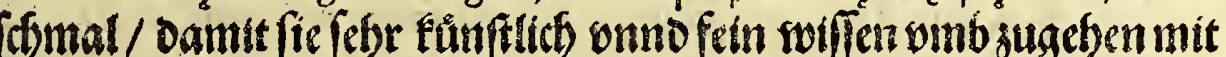

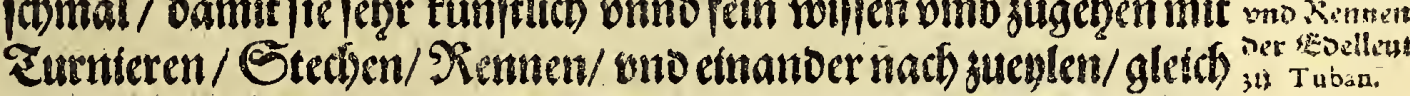

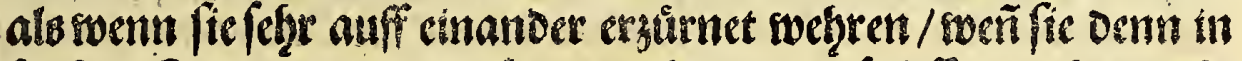
foldem Eurnieren bart zufammen fommen / foleffer ber binoerfe ber Den andern nadienlet/fenn Epieffinden/ono reiternebest oem anderndin / Dafa er vorifin fommet / oeñ gibe es feinem Pferot die 


\section{Drientalifchen Sintoien.}

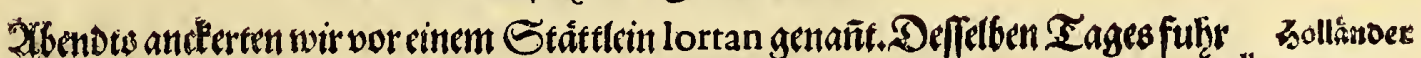

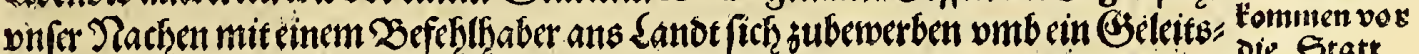

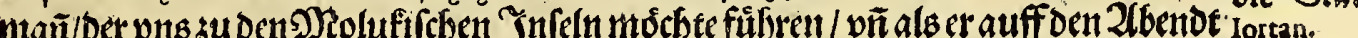

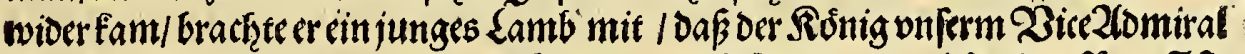

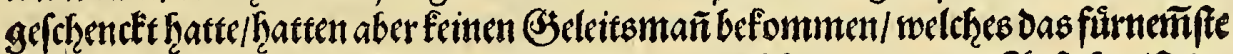

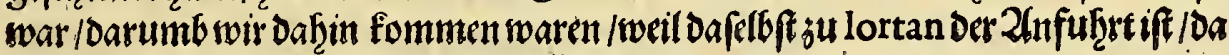
oic loncken oder $\sigma_{c}$ hiff von laua pflegen ju liegen.

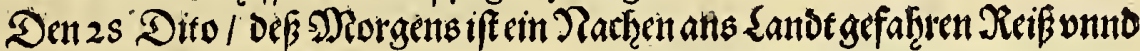

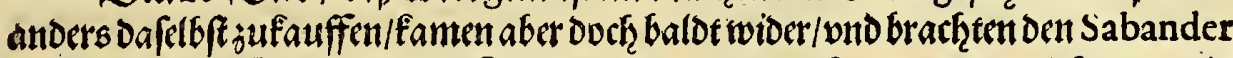

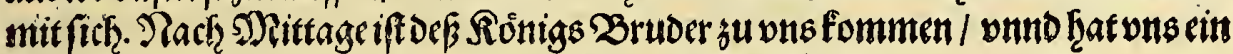
Dereffrung gebracht/Die et Dem Dite2lomiral fetencket.

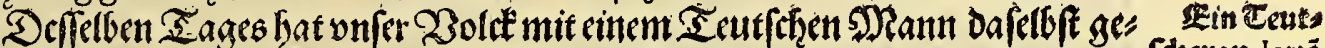

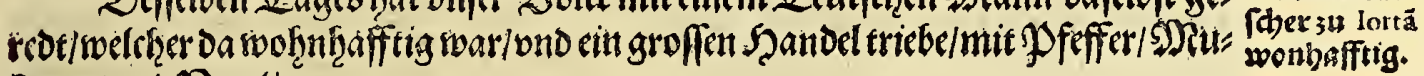
Fraten/onio Negelin.

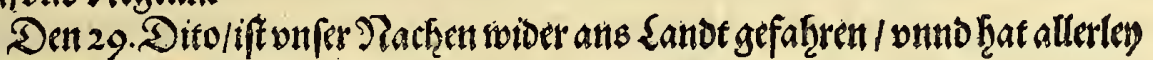
estentpeifecinfaufft

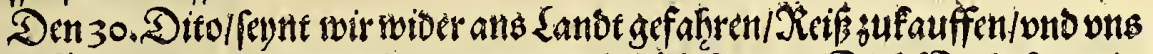

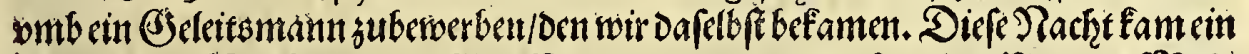

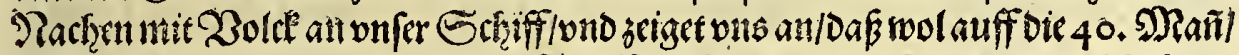
bon Dem 2lomiral wnd dem andern Echiff Sticht von Vtrecht genañt/züMadura, gefangen rweren/in einem Státflein A rosbay genannt/weldthes feffer fefferwar.

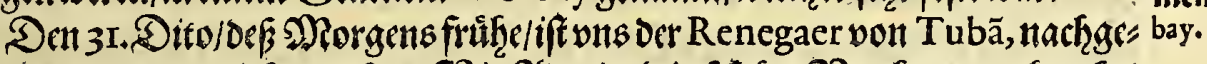

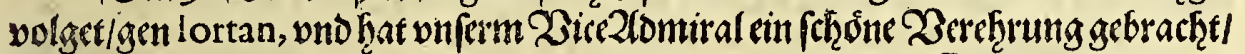

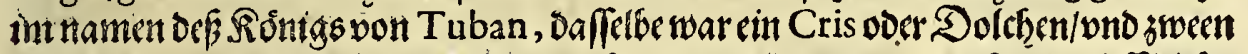

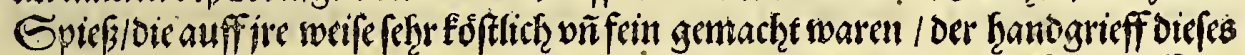

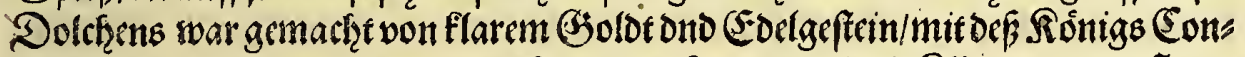

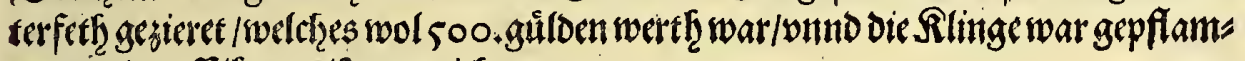
met/vno auffiffire meife zugerict?t.

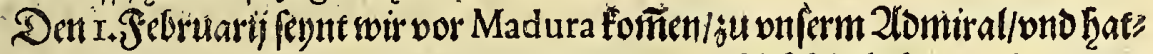

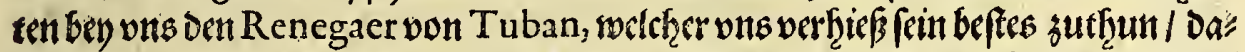

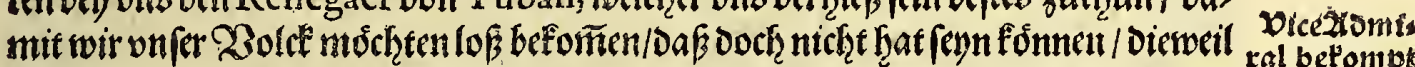

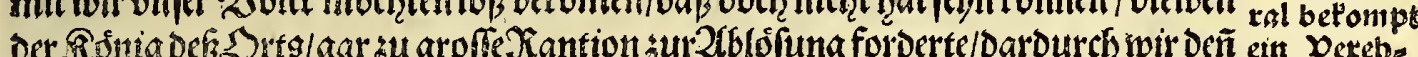

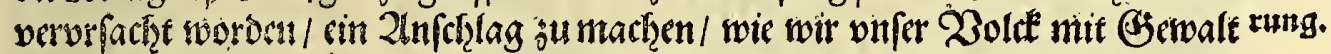

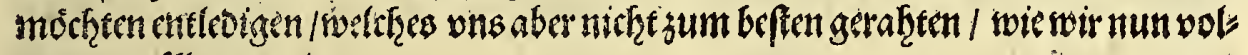
gendets wolletrianjetgen.

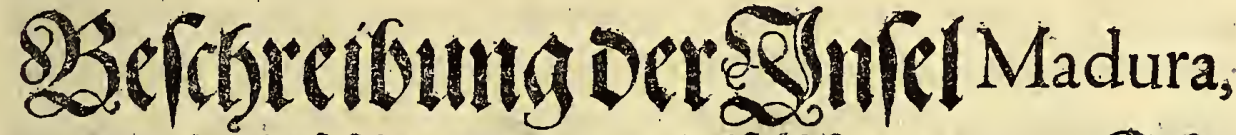

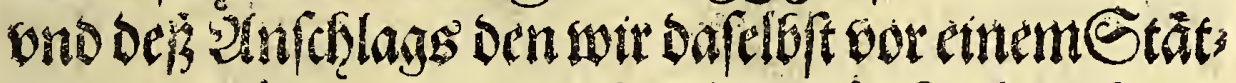
Icin A rosbay genañt/gematht baben/onfer Boldt/arldbes

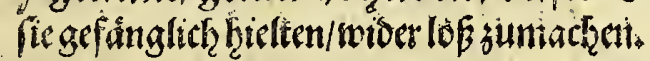

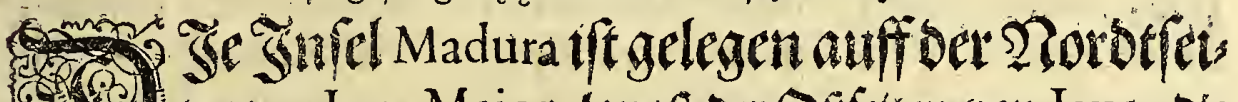
ten von laua Maior, langf der Dffetern von Iaua, ote (Ennfoghner gebengeflendet fote of \$u laua, feont gar ftu

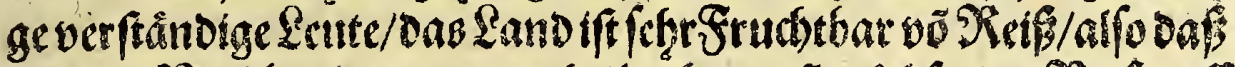

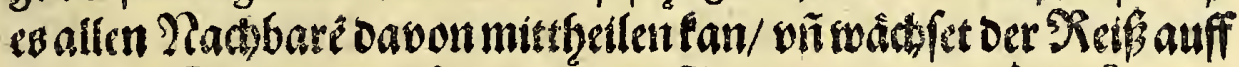

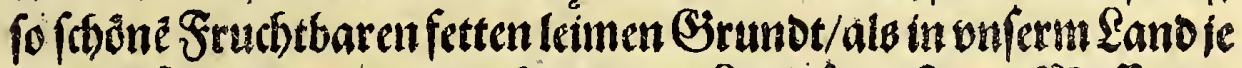

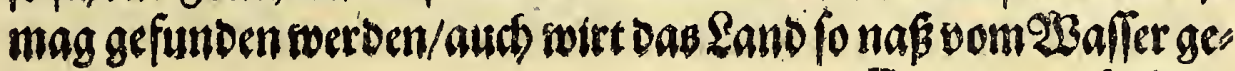

$\mathfrak{S}$ iij balten/ 


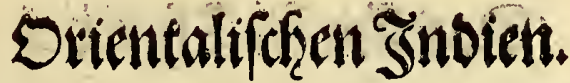

Sedis ono anbers fo vonnoten/oa/elb/t zufauffen/2llb/ie aber and Eand foumen / bat mainfie alle gefangen genommen/fbnen/f̧re

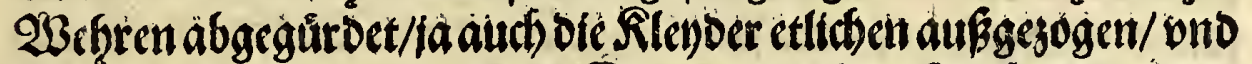

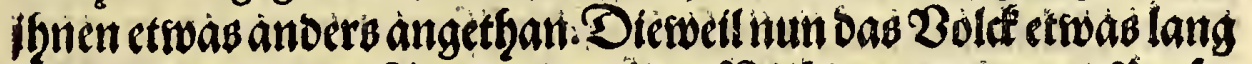

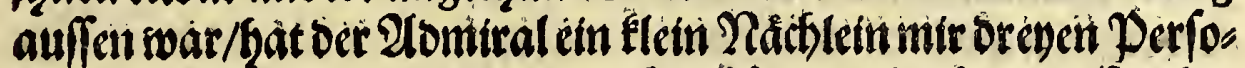

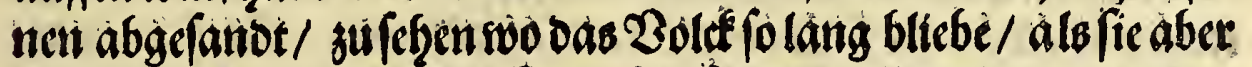
ans Lanot fainen/sourben/fe auch gefangen gentommen / onno su

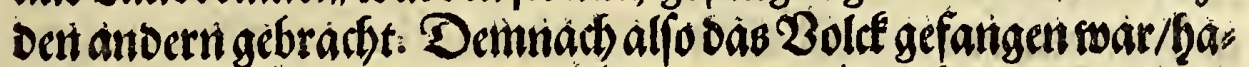

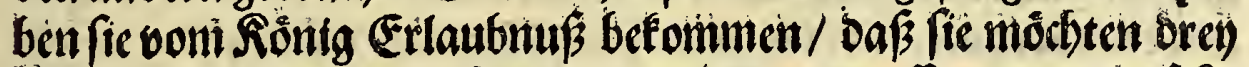

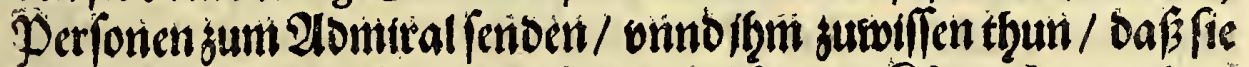

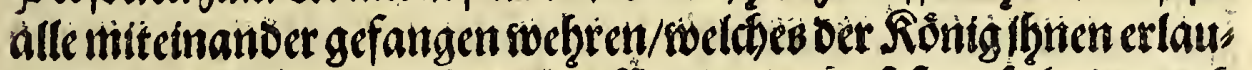

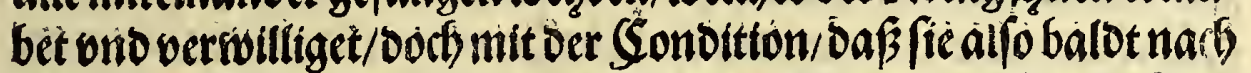

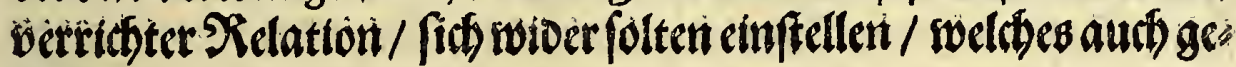
fit)ebert.

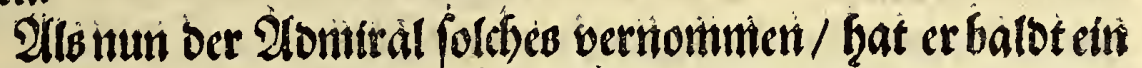
Siactengen Iortan abgefertigt / folches Den Bicediomiral zu bes.

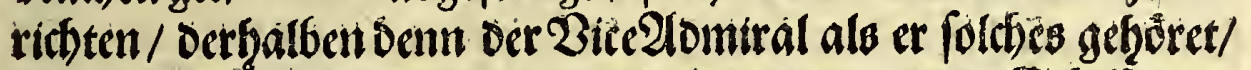
Das Alnufer auffectogen / ond àlobalot zu den andern Seniffenge"

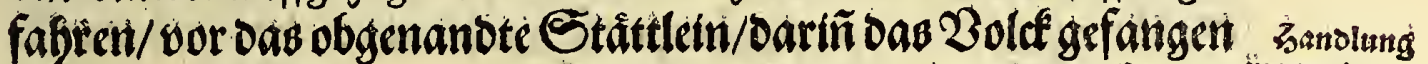

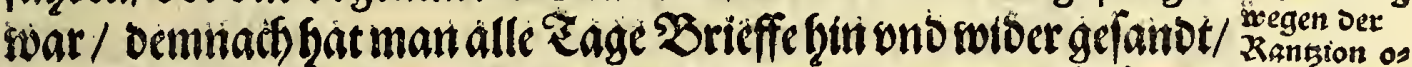

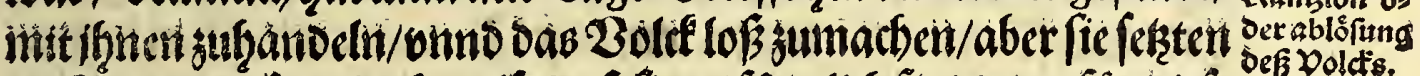

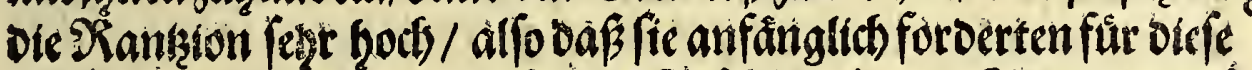

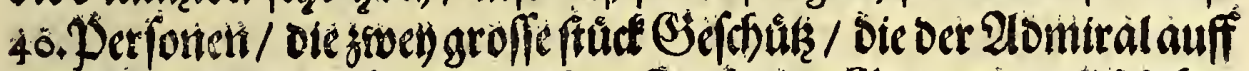

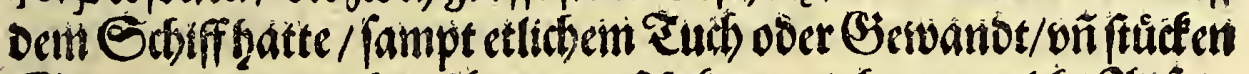

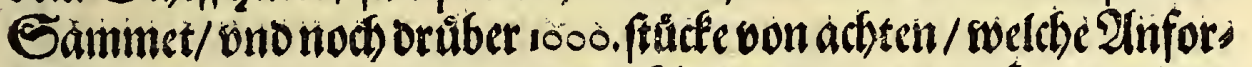

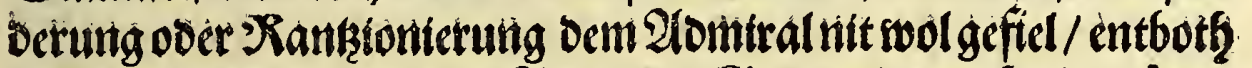
inen berbalben / fo fie mit Bjelt ooer Giut wolten bufíteden fenn/ soölle er gern mit ibiten frandeln ono Accorbieren / aber was bas

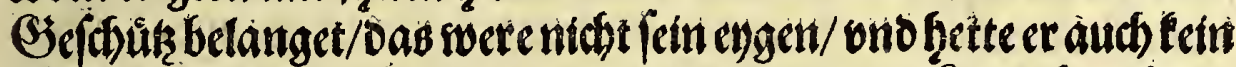

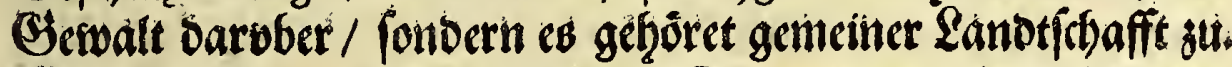
Diefe Shandlung fat wool 5.00er 6. Tage an etnander getwebret/

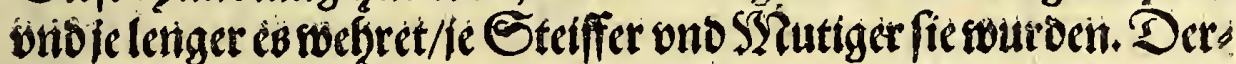

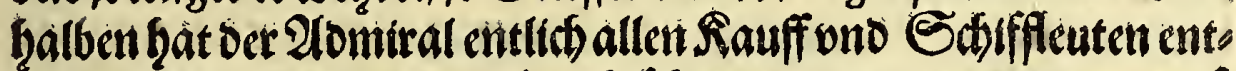

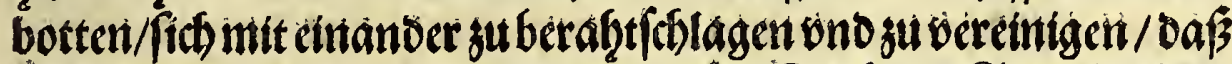
fie wolten ans Sanot fafren / vno viner Bolé mit Geswalt abho:

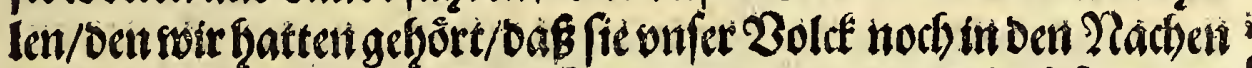

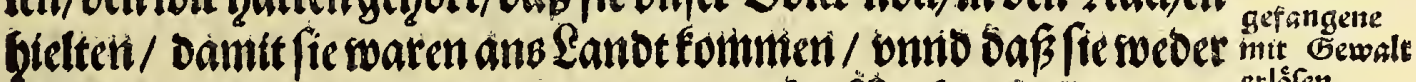

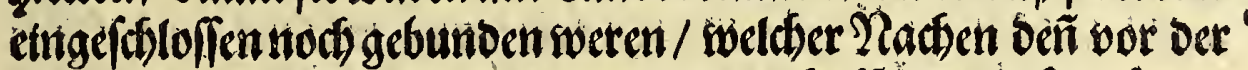

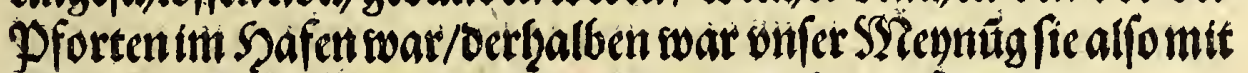
SSefzendigeeti Io

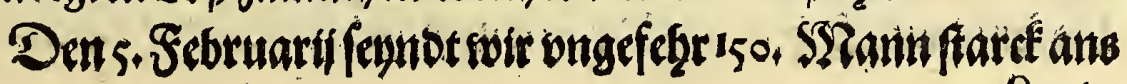

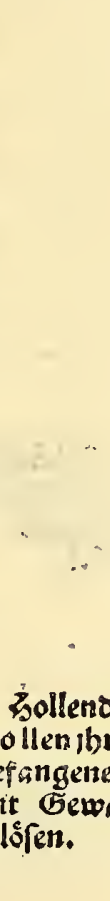
Sandt 


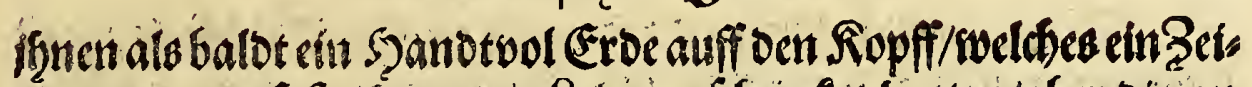

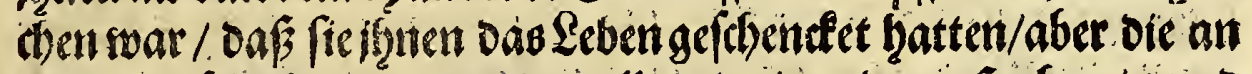
Die ander feite fancm / sourben alle mit einander erftoden / onno

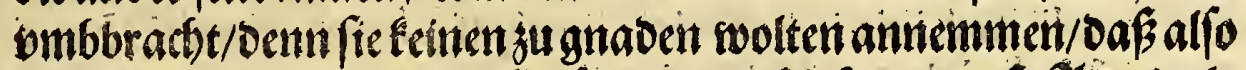
Damais soot blefen 25. Perfonen/nentblich von bef 2lomitals

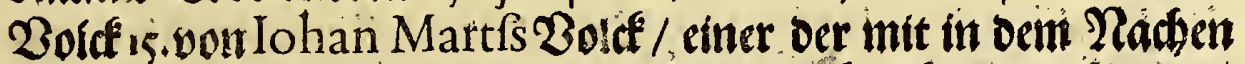

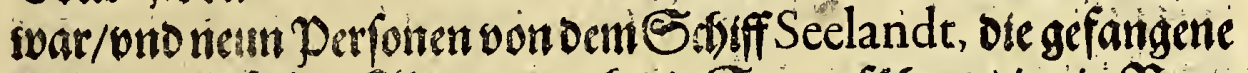

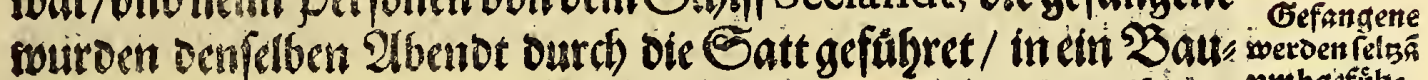

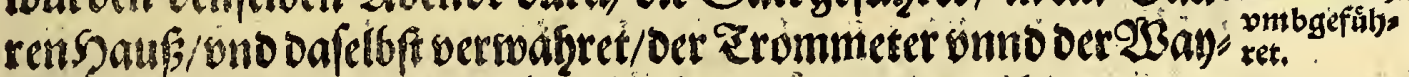
bel/wurben in enferne Sande gef(h)lagen/bie andern aber swurden alle mite einanoer gébunden / onter swelchen oren Perfonen obel

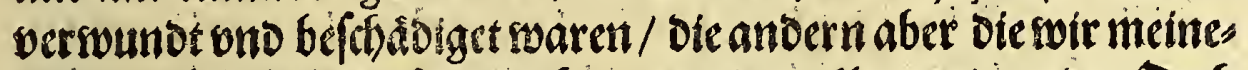

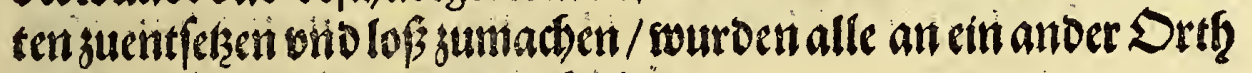

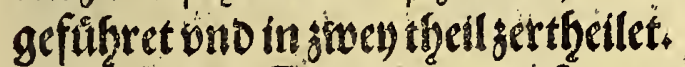

Peit 6. Dito/hat man fie von banten gebradit/ onno alofic

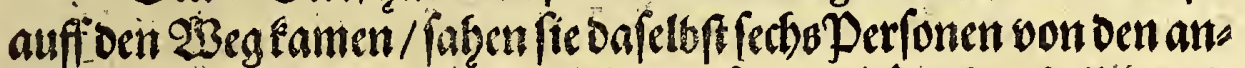
Dernabgelonbert/Die warenalffe ein befondern Orthgeftellet/ond

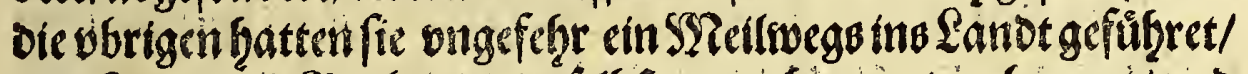

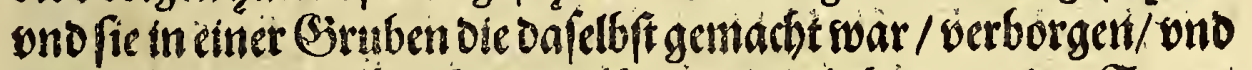

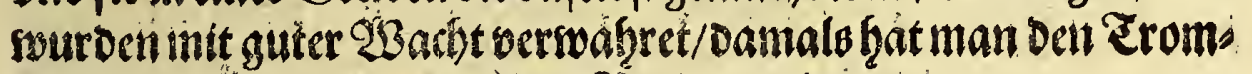

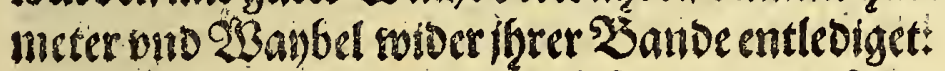

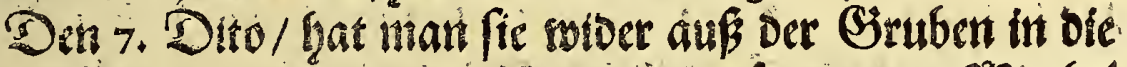
Etatt geführet / wno sward oer Rromineter fampt Dem $23 a y b e l$

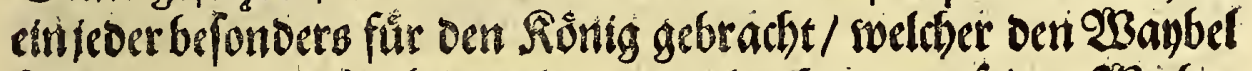

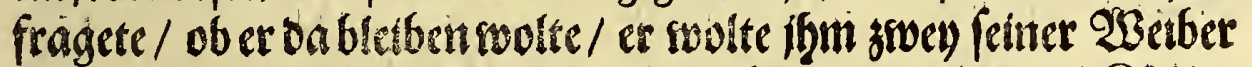

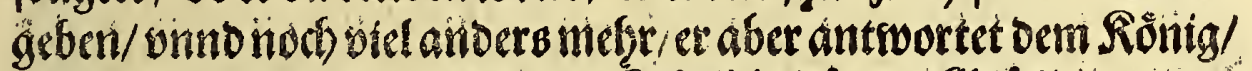

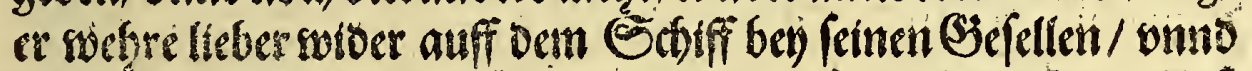

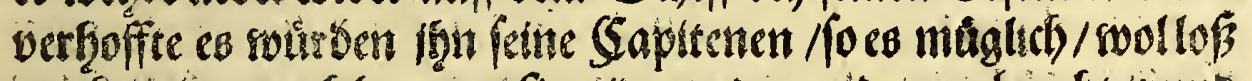

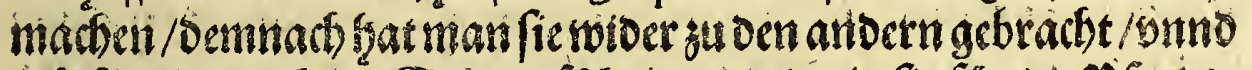

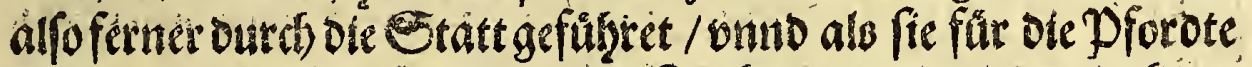

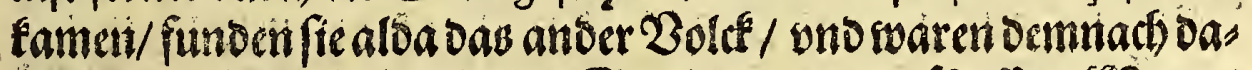

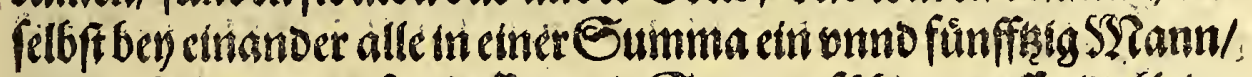
Darinath hat man fie culfer der Statt gefuffiret aufi ein tecine

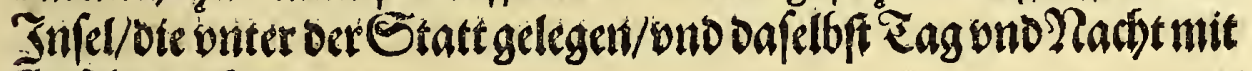

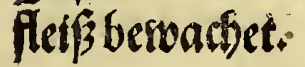

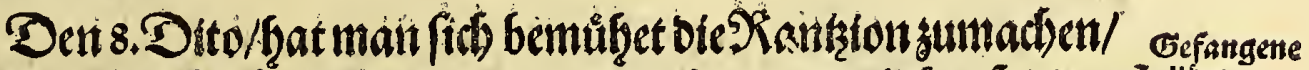

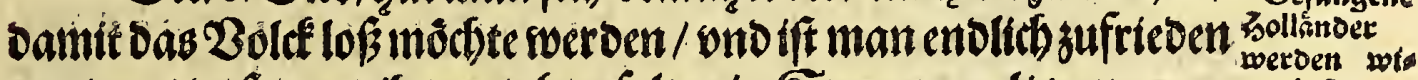
sworben / oaf mant fignen geben folte ein Summa gelt von 2000. Der log.

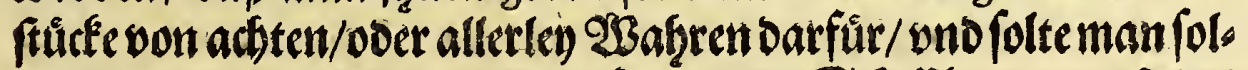

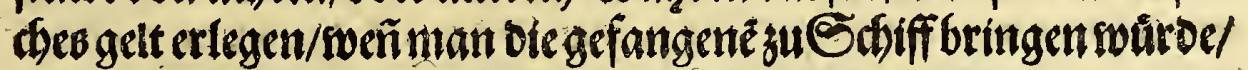

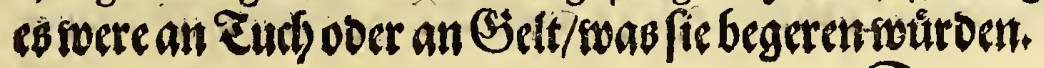

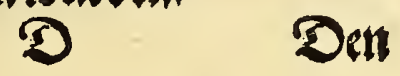




\section{Brientalifęen ̧noten.}

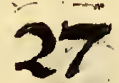

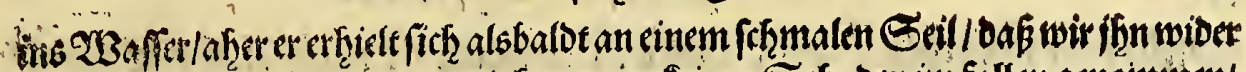
ints Echiff holen fonvten/ vano hat er gar feinen Echaden im fallen genommen/

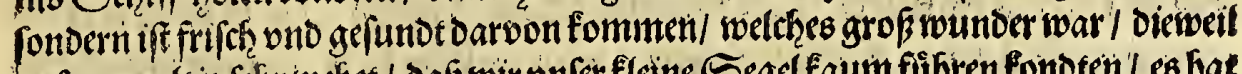

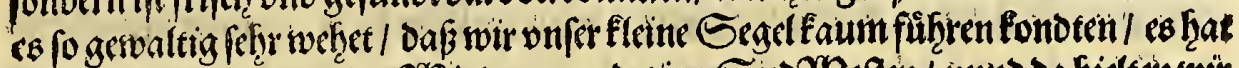

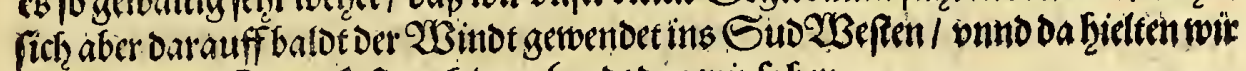

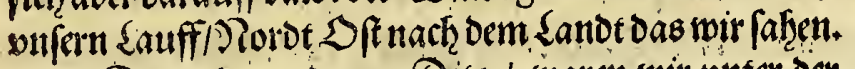

Den 26, vnno 27. Dito / waren wir onter Der Infel Blau ine fitle ges

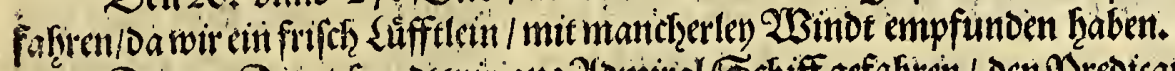

Den 25 Dito / found wir ans 2lomiral Schiff gefaftren / Den Predicanten

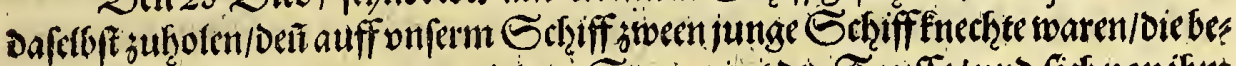

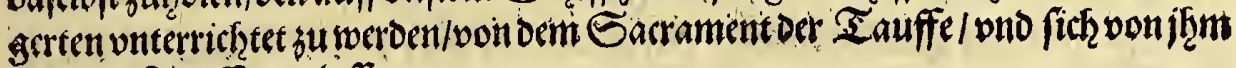
hernach rauffen julaffen.

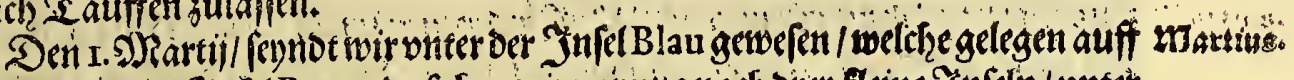

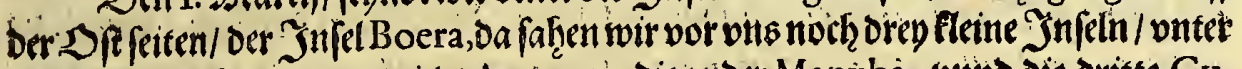

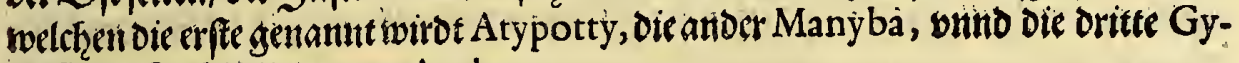
ta. Diefe Infeln liegen vor Amboyna.

Den 2. Dito / fennoe wir vor bent Sanbe ber Infel Blau, votuber gefafren! unino gegen bem 2(bendot / Eamen jwo Prauvven an Den 2lomiral/fie wolten aber

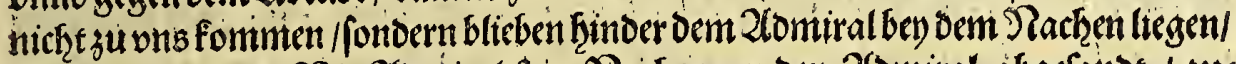

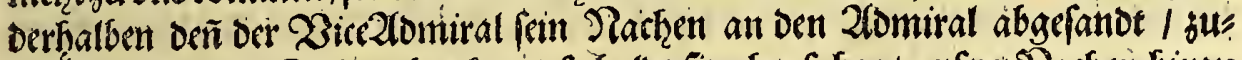

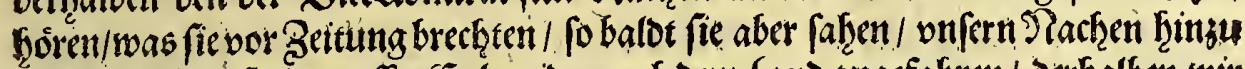
fommet / feunfie in groffer Enlewider nach Dem Sano zugefaftren / Derhalben wit onferm Sauffoollents nact A mboyna genommen.

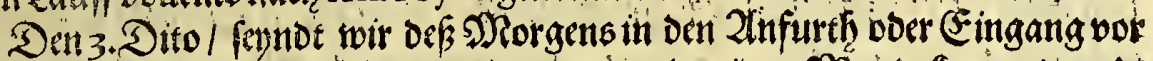

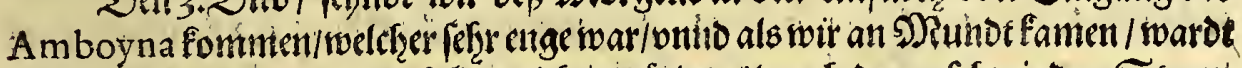

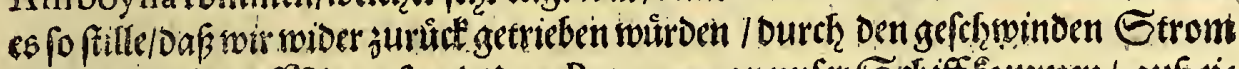

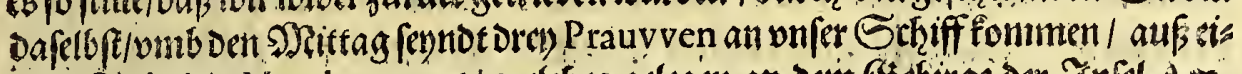

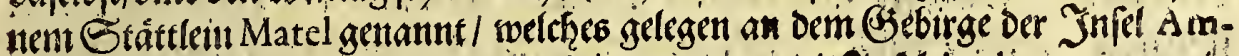

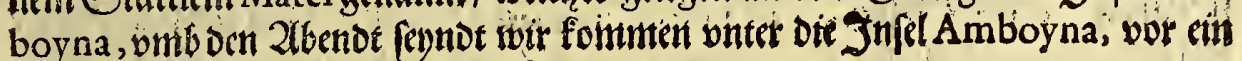
Etátfletin gentantnt Itou ober Iton.

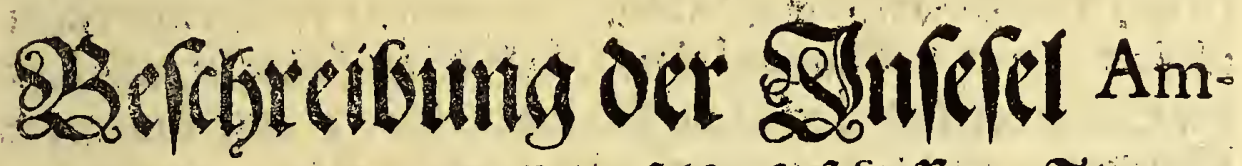

boyna, wie nemblich biefelbe befchaffen / Gtem

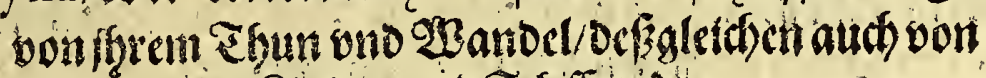
ihrer filtenoung vind Schiffen oder

उalcen.

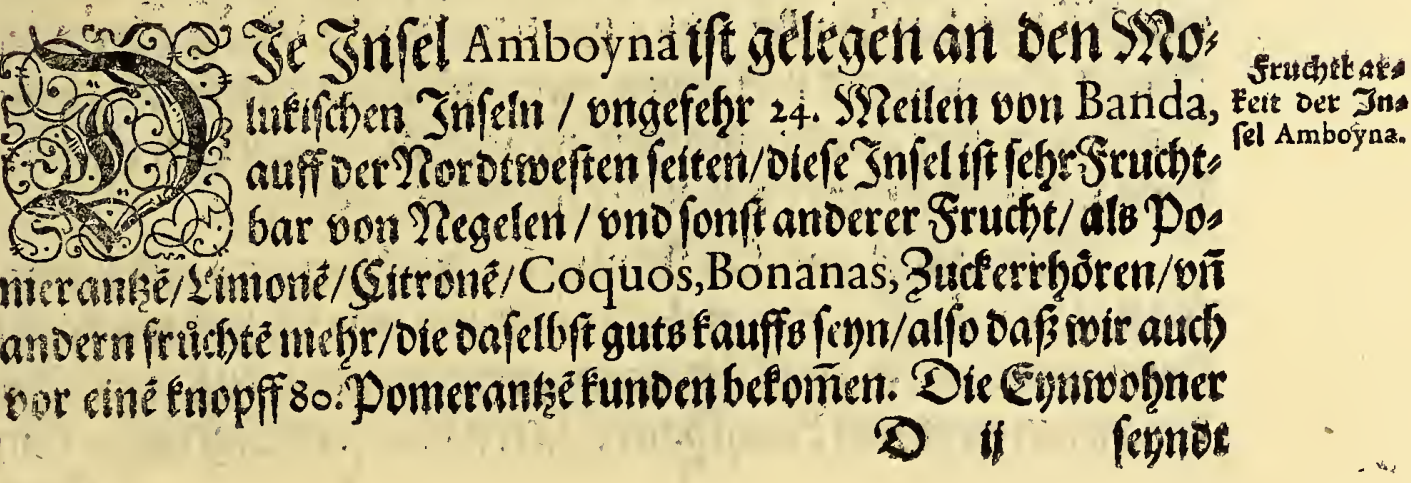




\section{Drientalifąen Snbien.}

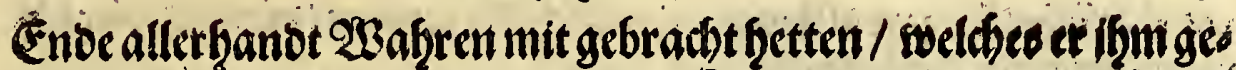

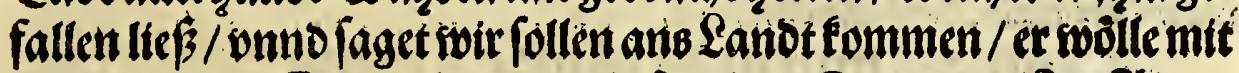
ons haanbeln. Derbalben dent de

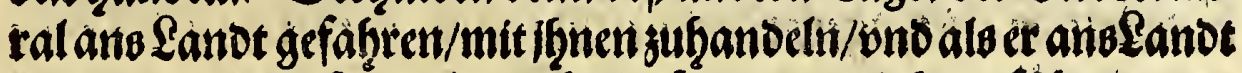

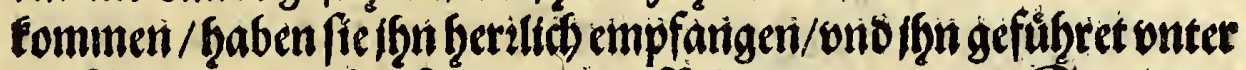

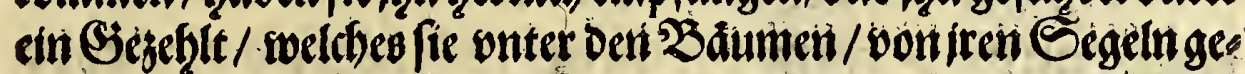

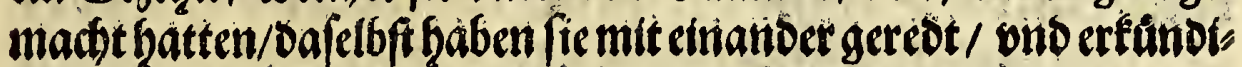

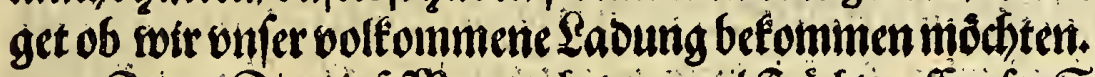

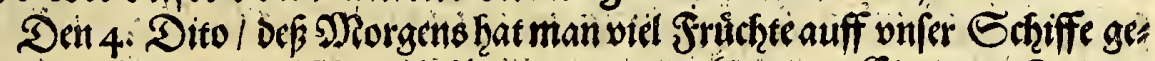

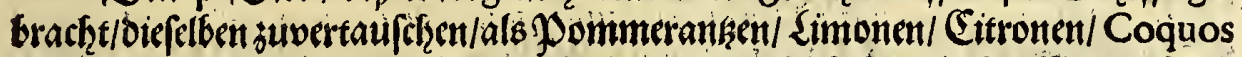

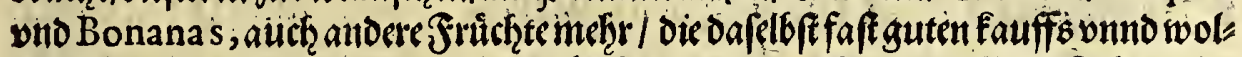

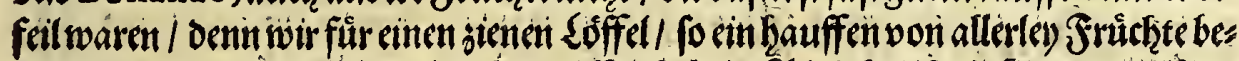

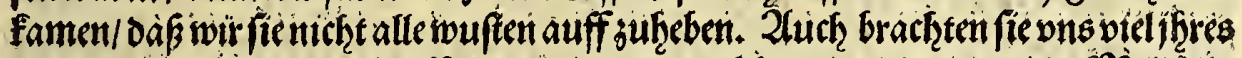

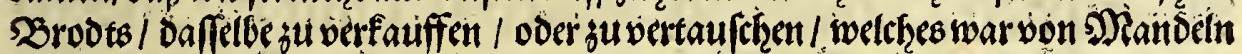

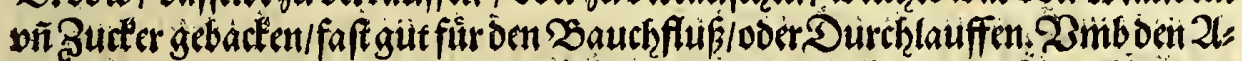

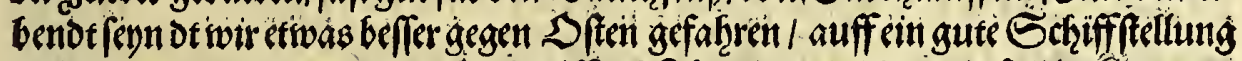

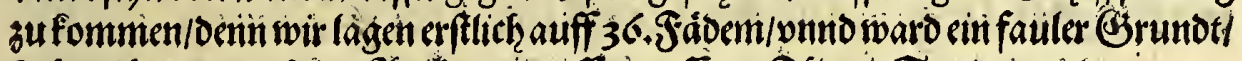

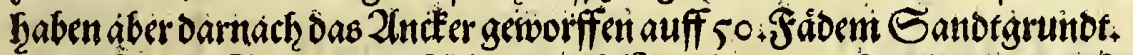

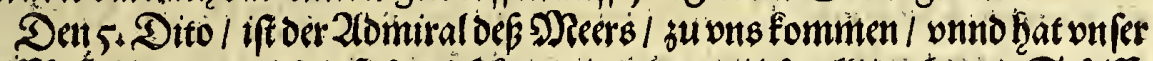

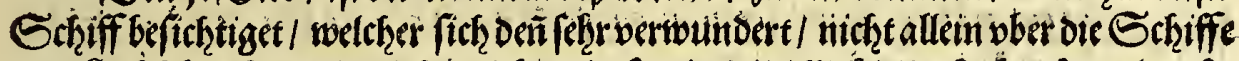

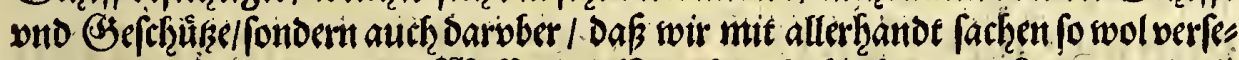

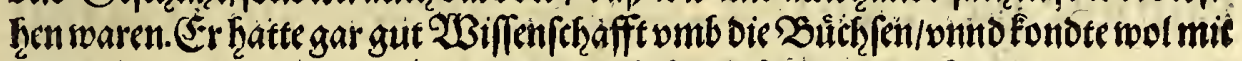

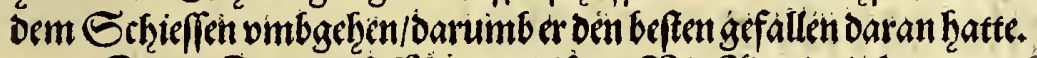

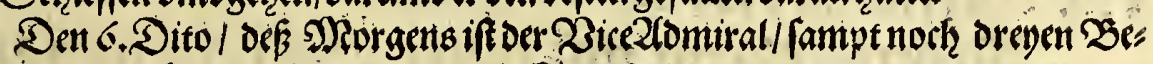

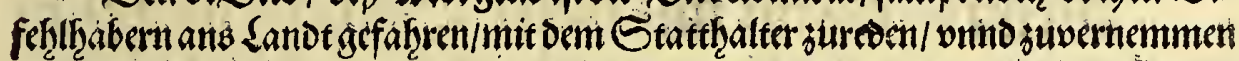

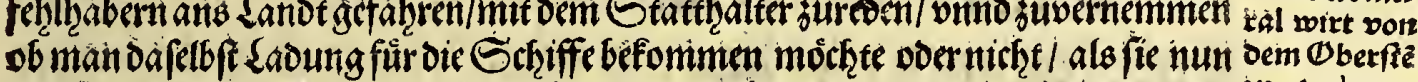

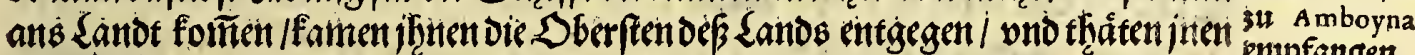

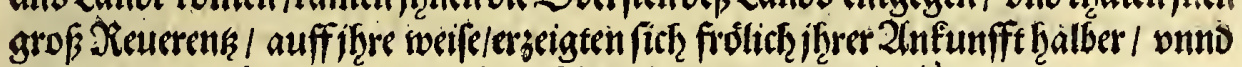

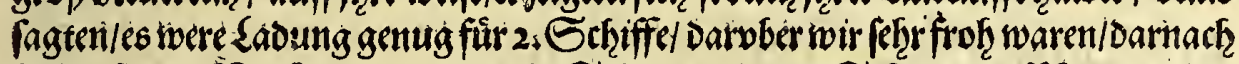

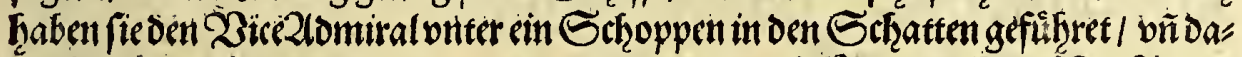

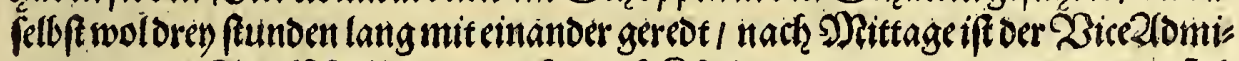

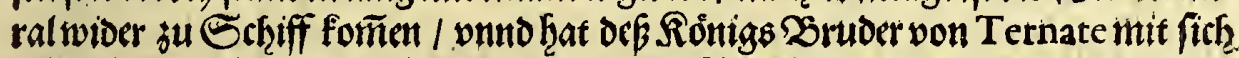

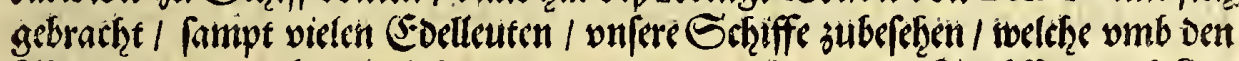

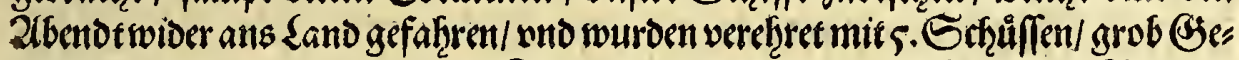

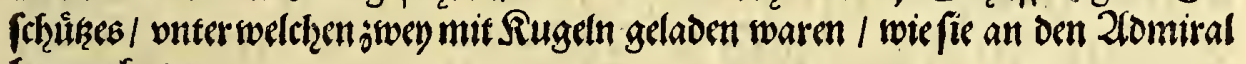
begeret hatten.

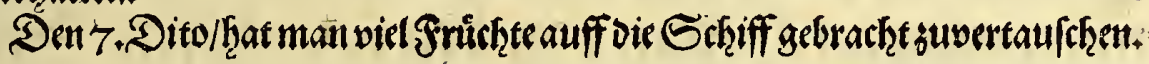

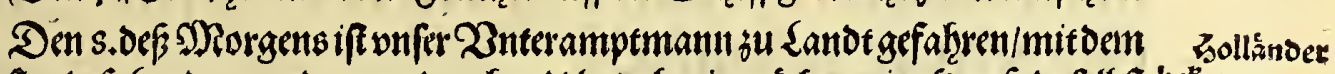

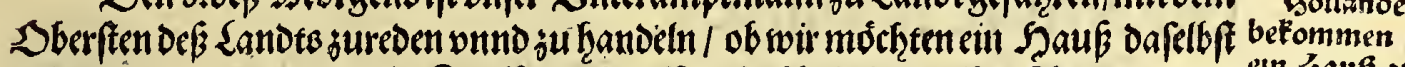

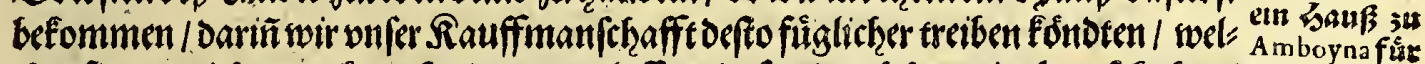

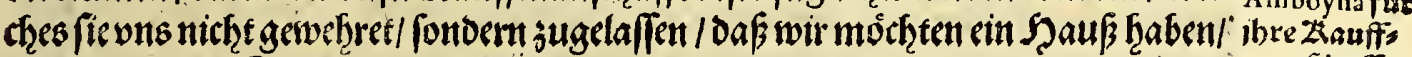

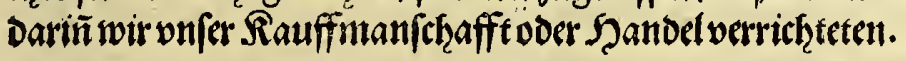
maindoaft:

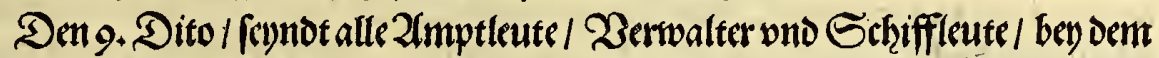

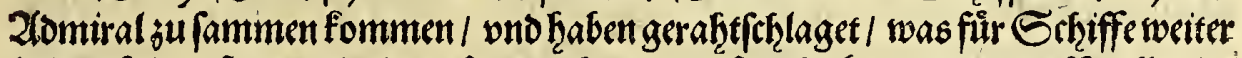

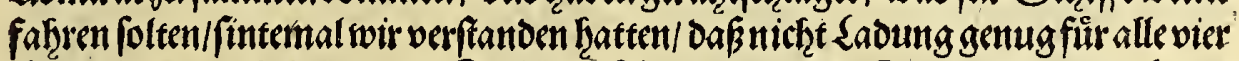

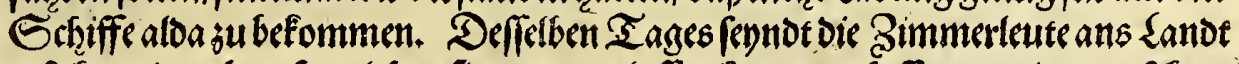

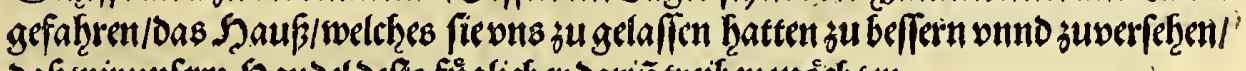

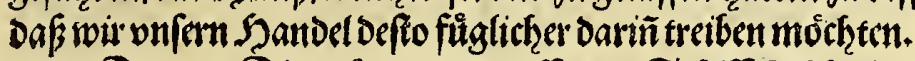

Don Io. Dito/ hat man auff Dem Sthiff Gelderlandt Ëerticht gethaltem/

D iij ono 
Doter Ddutfe oex Jollsins ver poerden

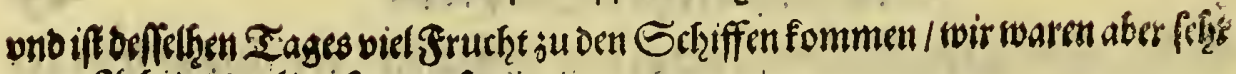
in ber 2Arbeit/das Sjauk ju verfertigeii.

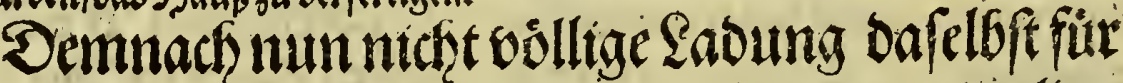
die vier Sebiffezubtommen / we broben gemelot/ fo bat man nach gebaltenem $R$ aldet/chlag / für gut ar:

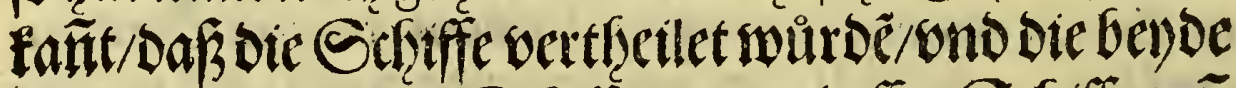

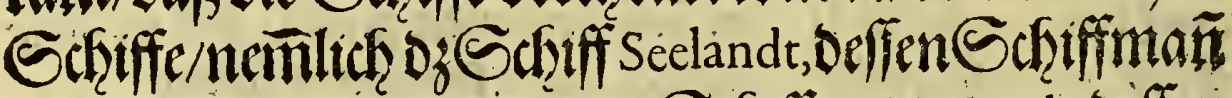
war Ian Cornelifs, wnno bas Sentffi Gelderlandt, beffen Sobiffman war loan Bruiin, nafer Banda faften folte) Derfalben wir mit genañten żwe) Schiffen Den II.

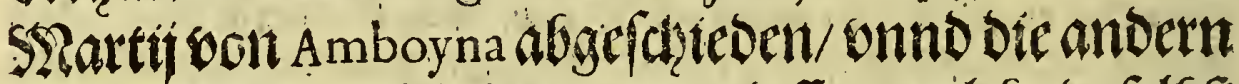

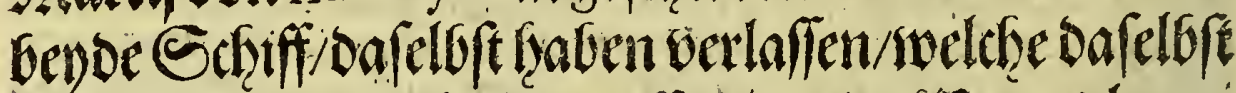

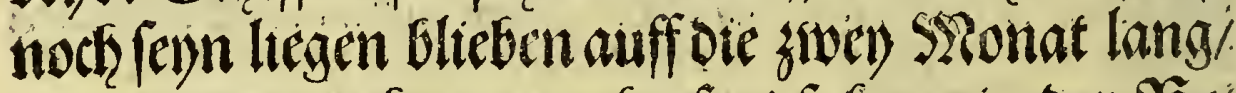
iwollen bemnach nummefir fort fafteren in der $\mathfrak{B}$ es

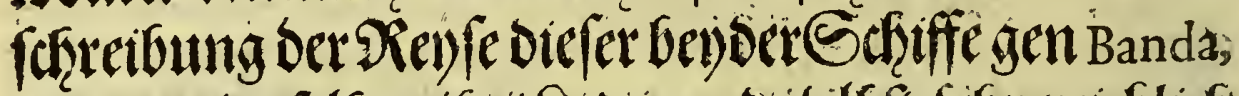

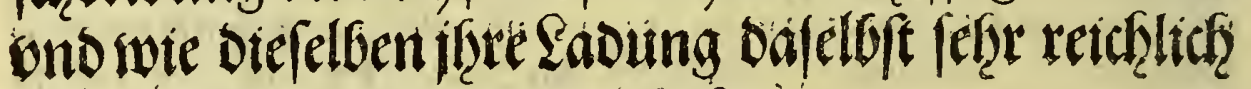
who werfluiflig Getommen fraben/

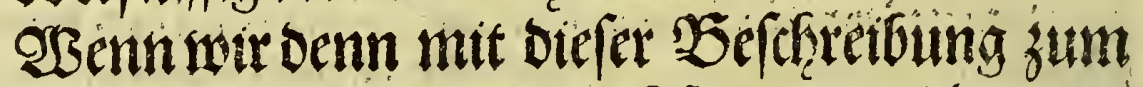
Ense fonmen / wóllen wir auth fernet erzedzlen/wie

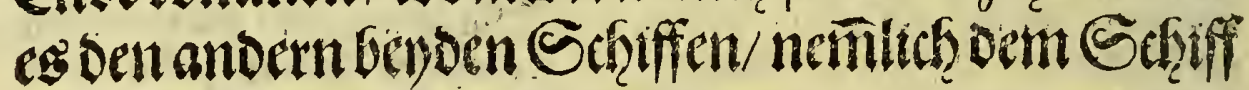

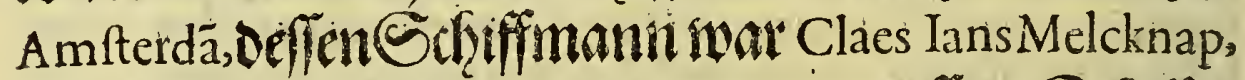
wnno bem Gefiff Sticht won Vtrecht, deffen Edriffer war Ian Martfs, fernet ergangent/welcfe won Amboyna; Den8. Sraij abgefaloren leyn nach ben Srolifteffen

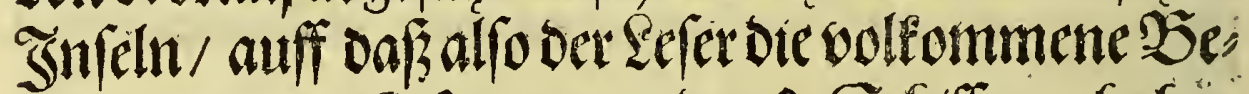
fertetbung oer Setftory won dens. Eefriffen/ fraben ond verneminen mag.

Den II. Sito/fenthot wir von ben anoern jweenen Schiffen abgefthicoen/onts

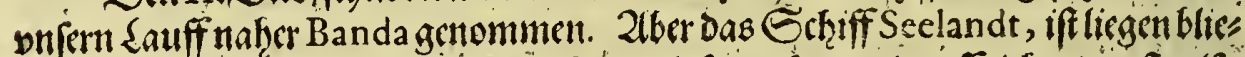

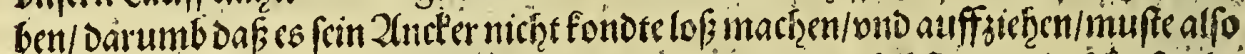

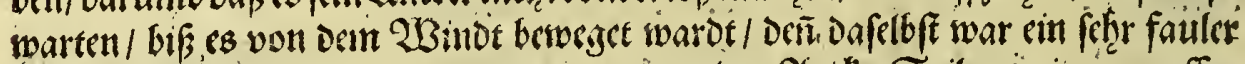

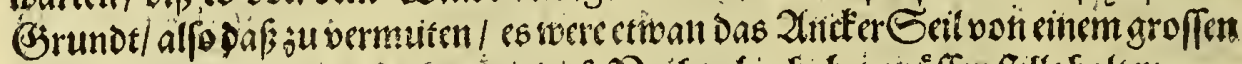

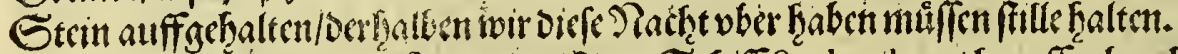

Den I2. Dito / oef Diorgens ifto as Echiff Seelandt auch auffgebrocticn/

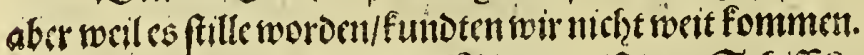

Den I3. Ditol gegen Mittag/ ift Das Gibiff Seelandt auff Den Grunbet foim 


\section{Srientaliforen Inteient.}

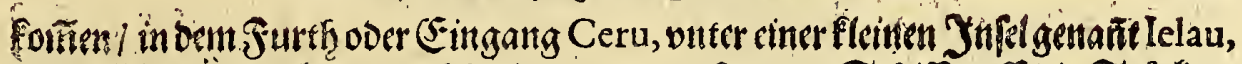

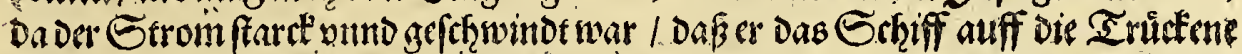

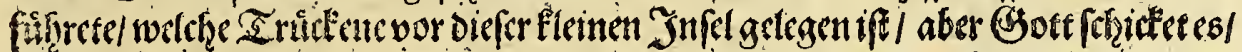

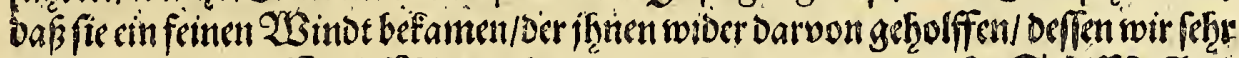

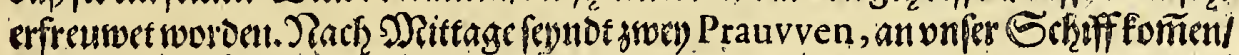

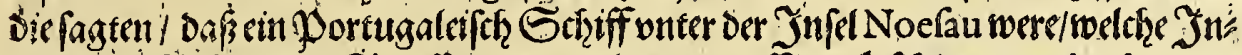
fel in Dem 2 infang Der Strafen Ceru gelegen / anff der Dfffeten bon Amboyna,

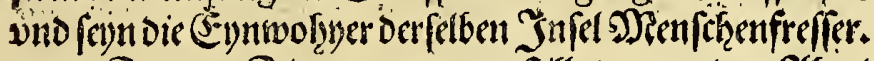

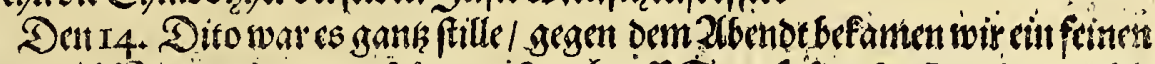

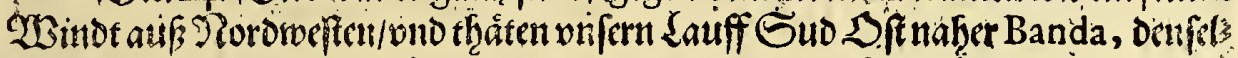

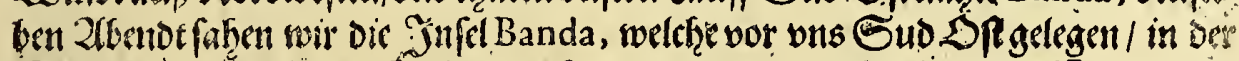

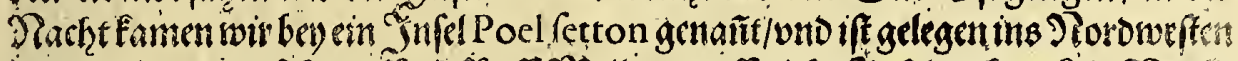

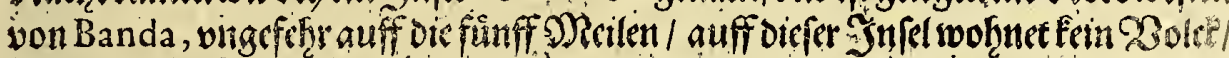

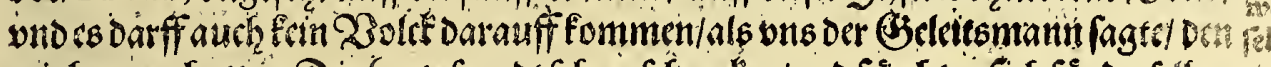

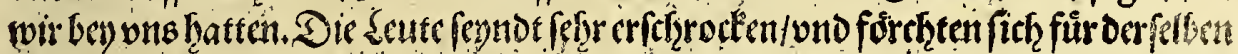

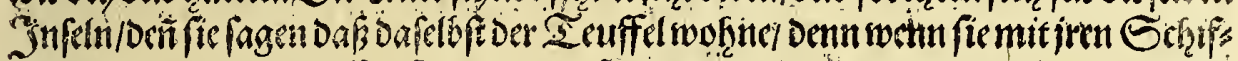

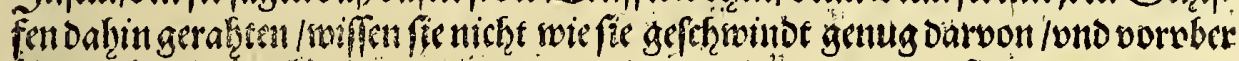

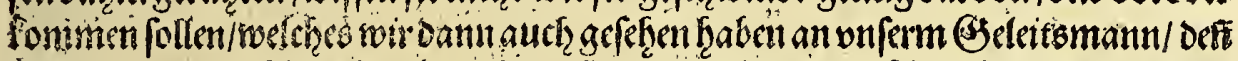

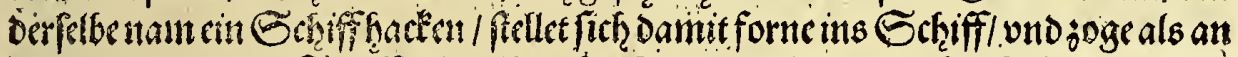

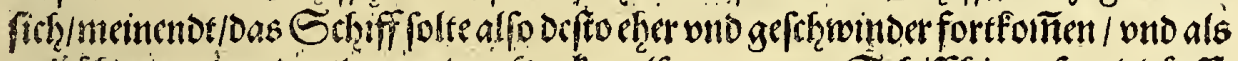

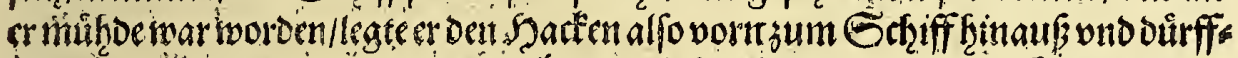

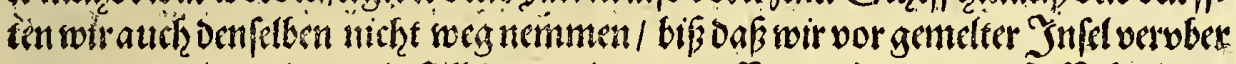

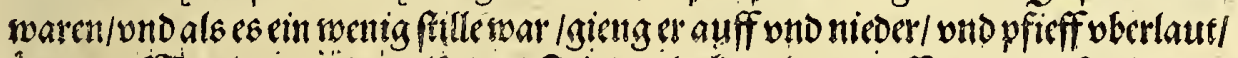

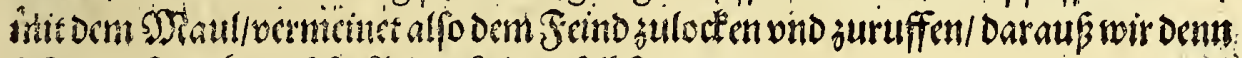

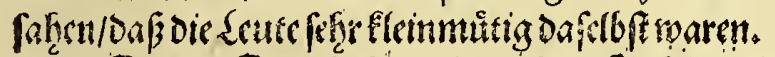

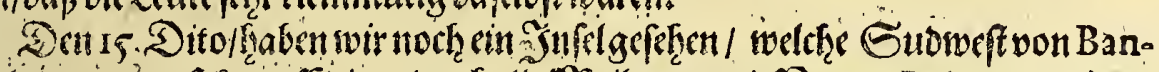

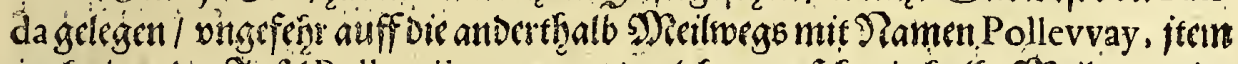

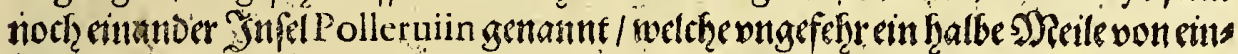

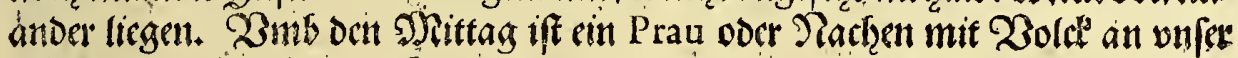

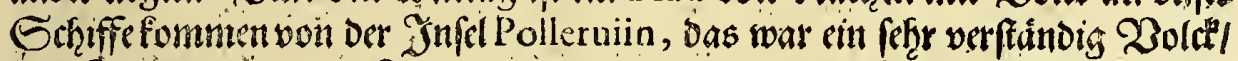

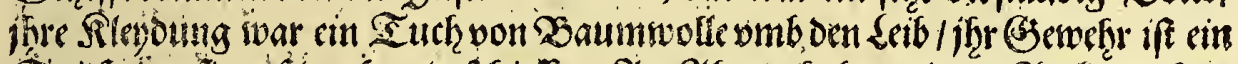

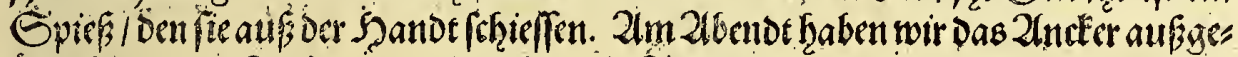

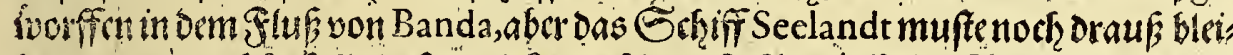

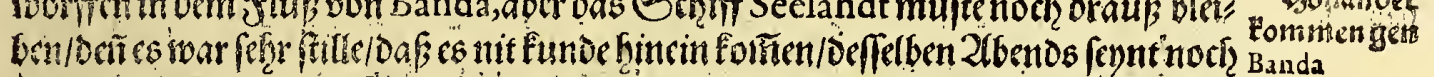

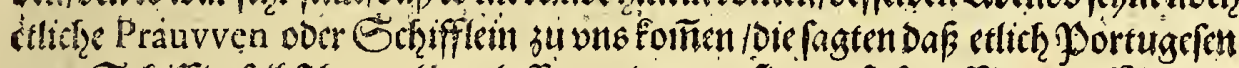

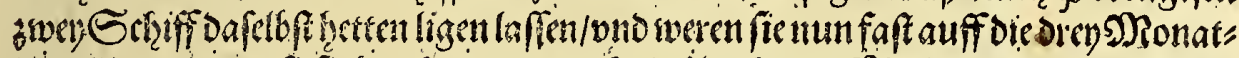

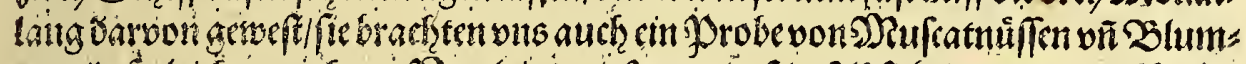

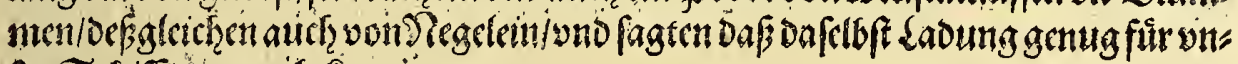
for Eefifferesere jubefomiten.

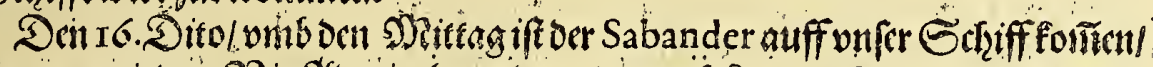

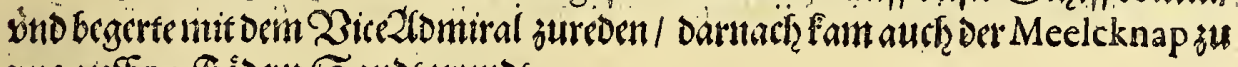
ons auff 14 . Findom Sandforundet.

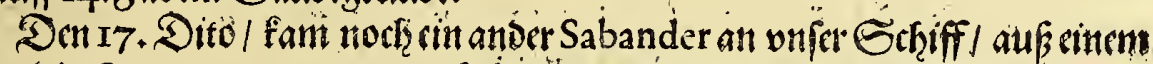

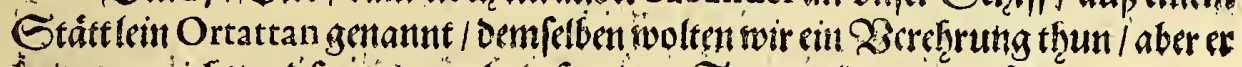

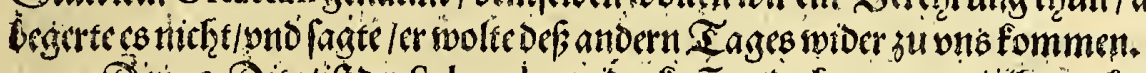

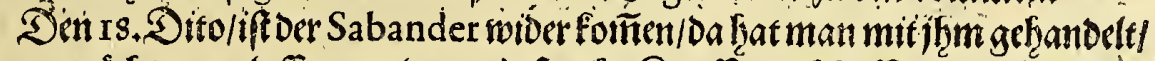

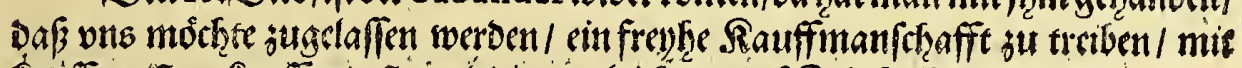

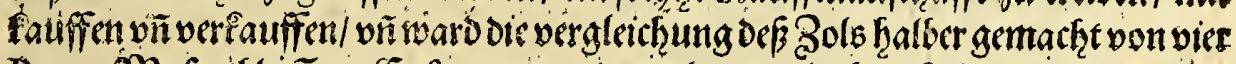

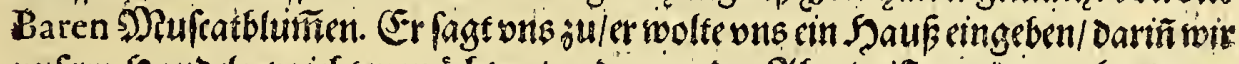

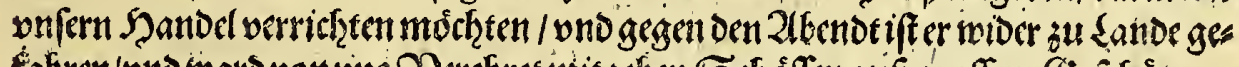

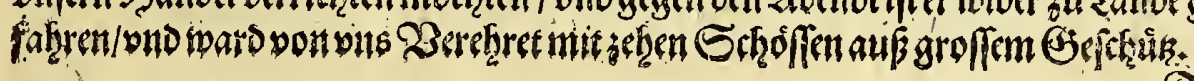




\section{Orientalifçen S̈ndien.}

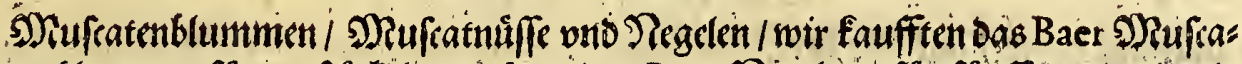

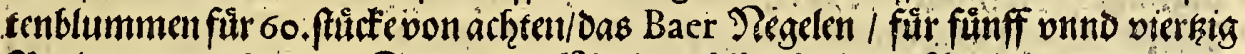
Realen won achten. Das Baer Mifeatuiffe fü fectzos Xiealen von actiten. (Es ift aber cin Baer hunibert Pfunde / Def Gerbichts ju Banda, ein Po fund

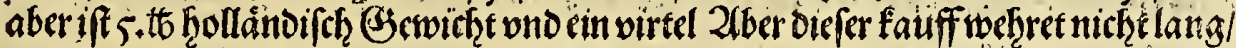

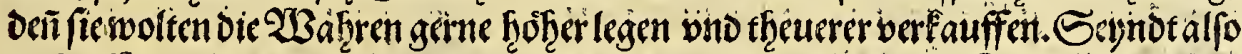

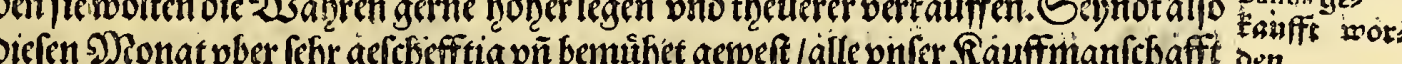

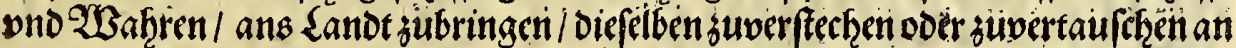

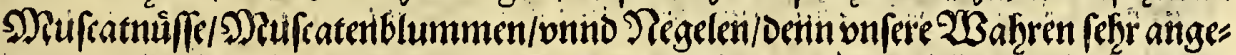

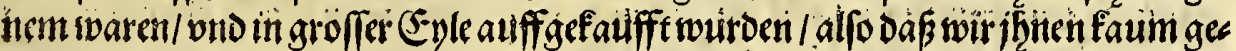

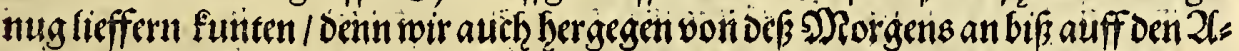

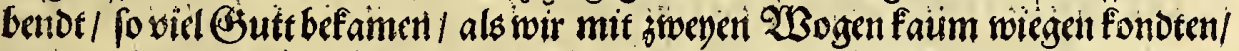

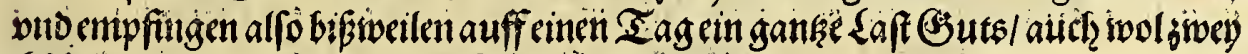

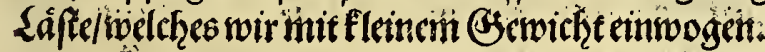

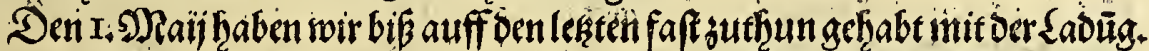

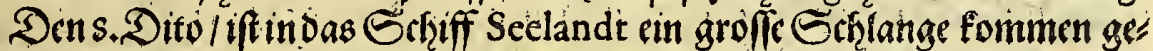

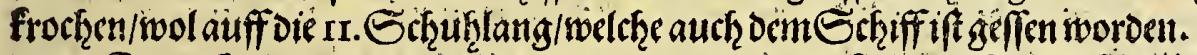

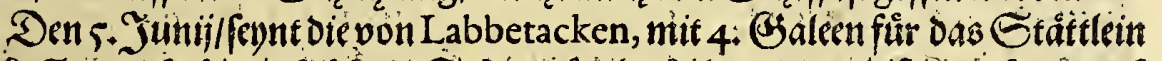

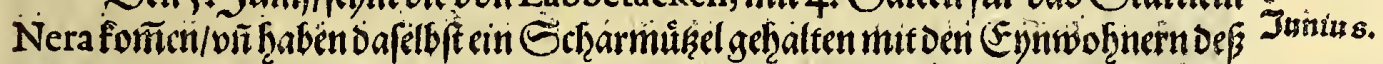

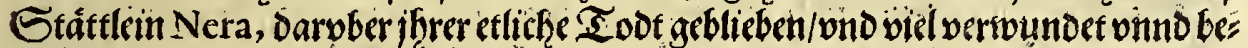

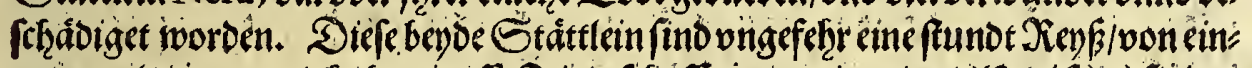

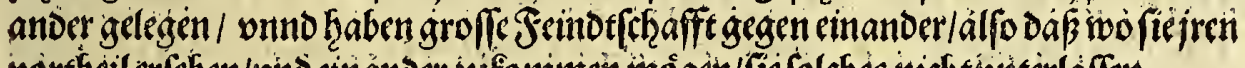

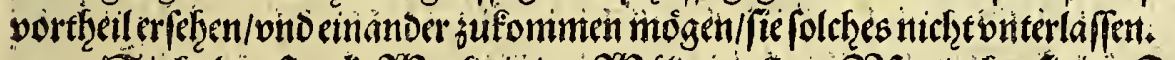

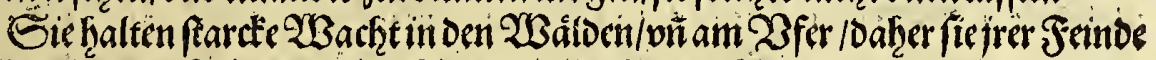

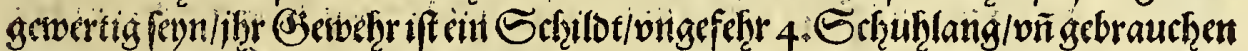

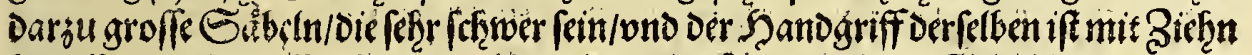

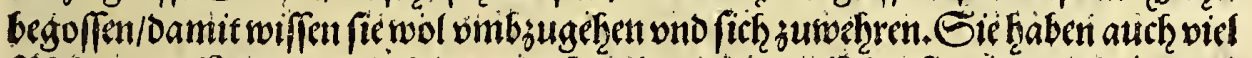

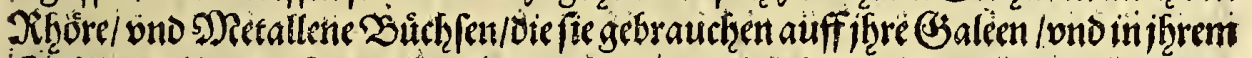

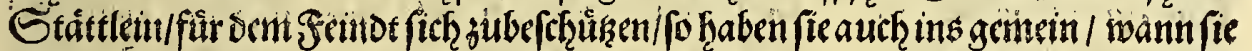

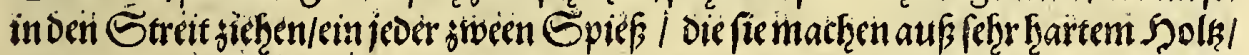

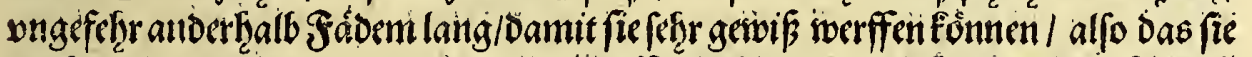

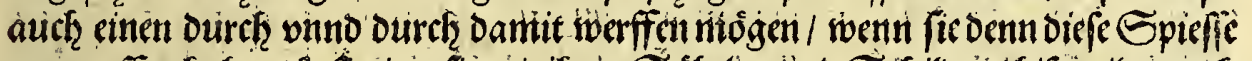

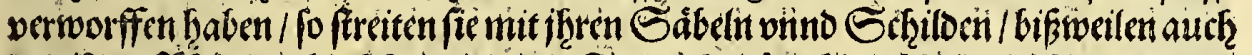

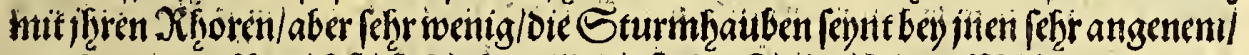

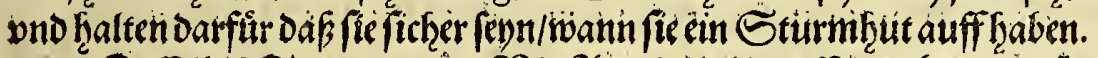
Gelteni.

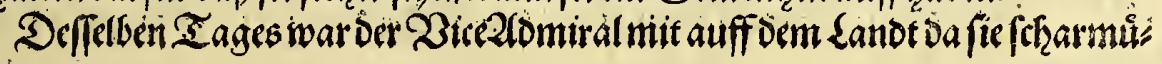

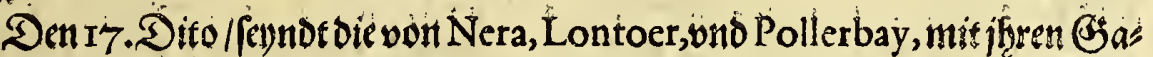

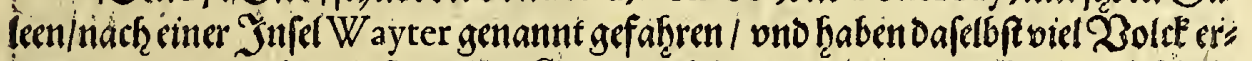

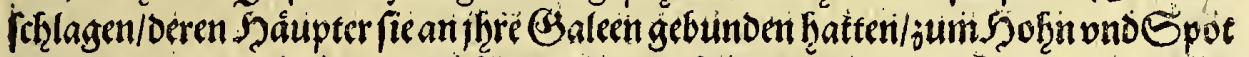

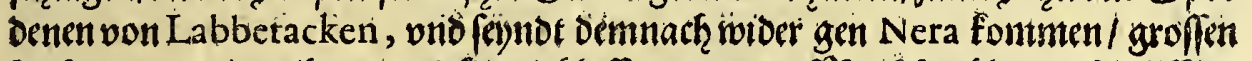

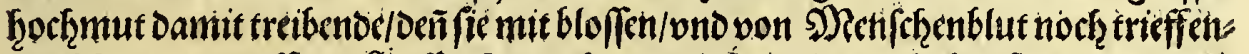

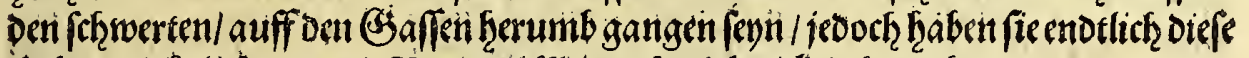

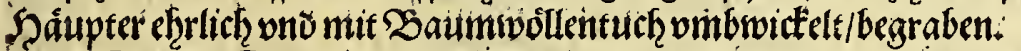

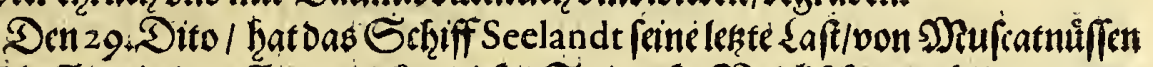

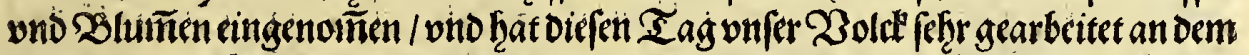

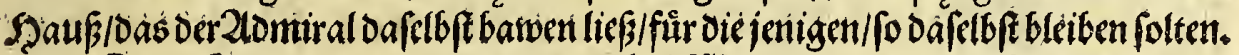

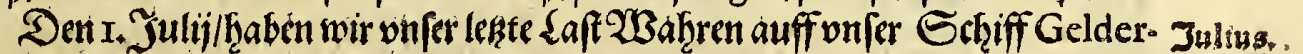

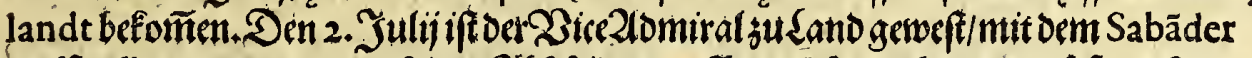

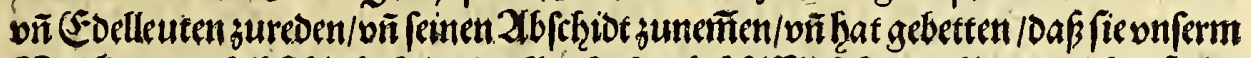

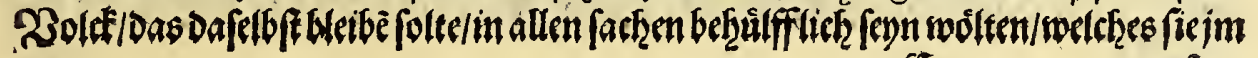
(5) ingefagt 


\section{4

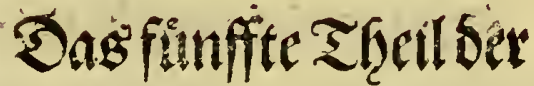

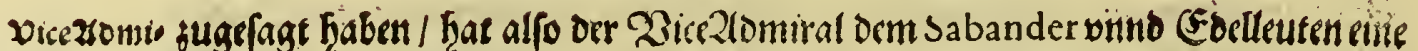

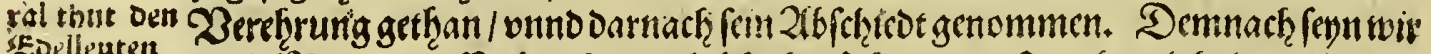

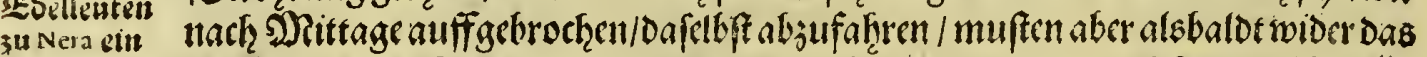

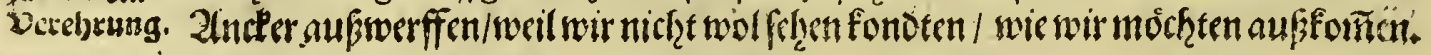

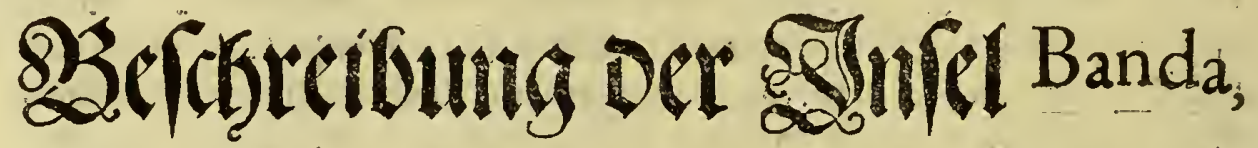

swelkfe gelegen auff 4, graben / halb in Guben won

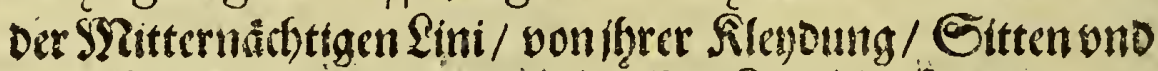

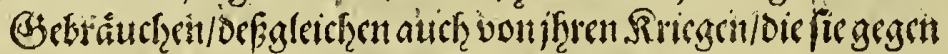

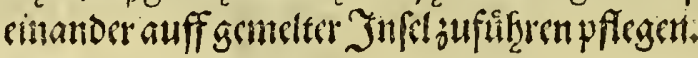

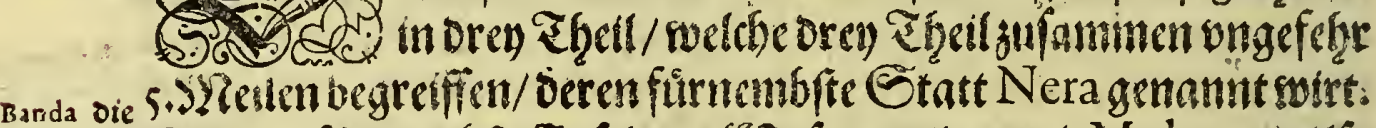

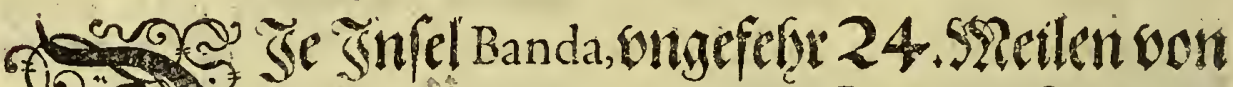

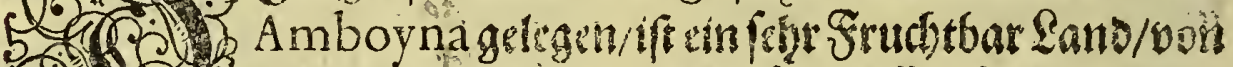

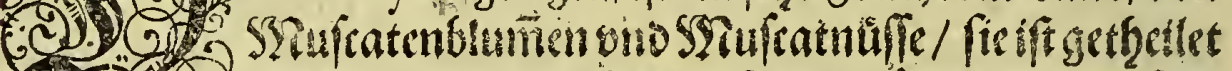

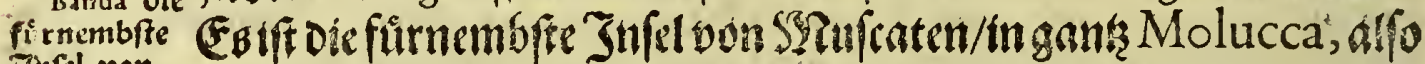

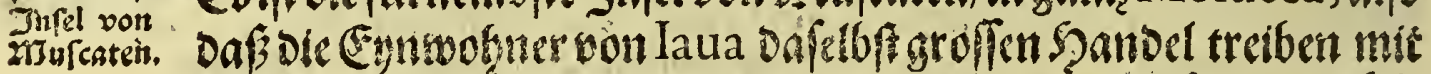

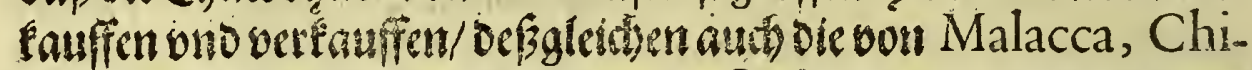

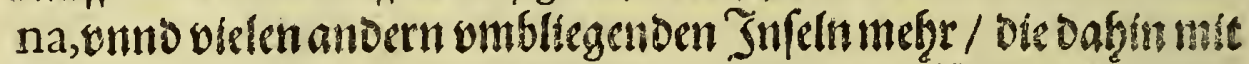

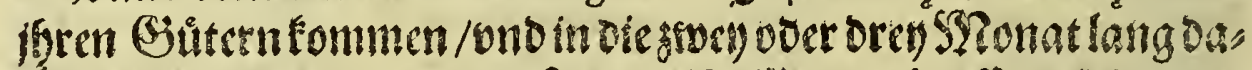

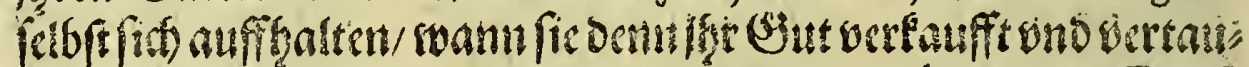

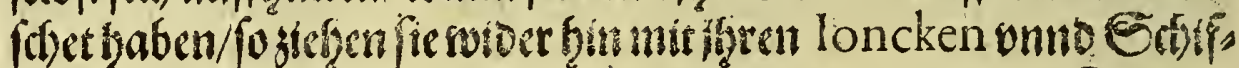

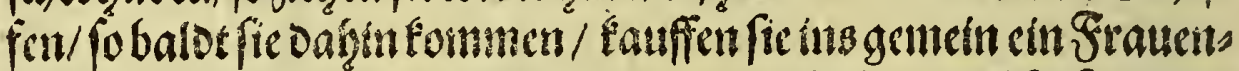

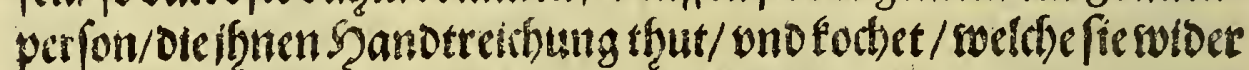

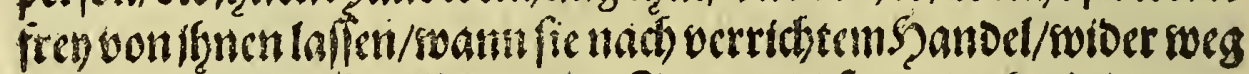

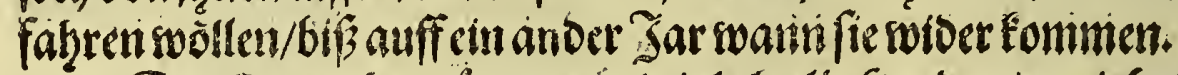

2งachunes trifbe Kidis

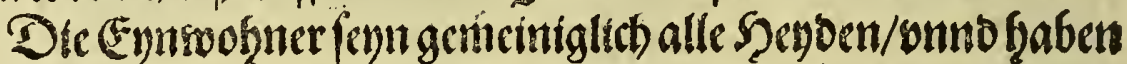

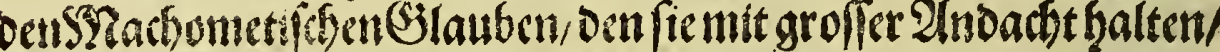

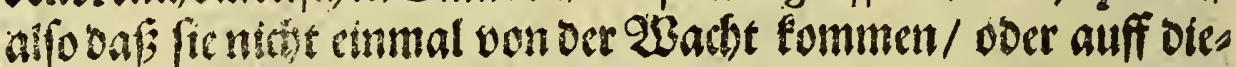

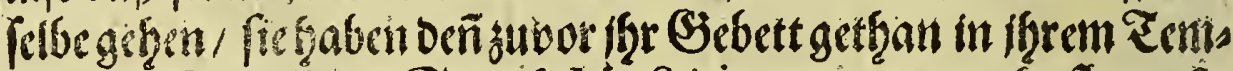

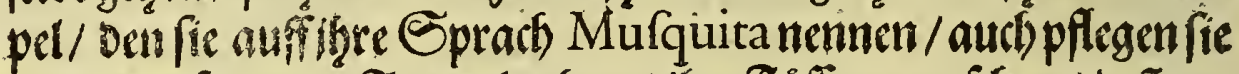
zuvor ebe fie in oen Eempel gethen / ligre Fúfle zurvaftoen / Deñ ges

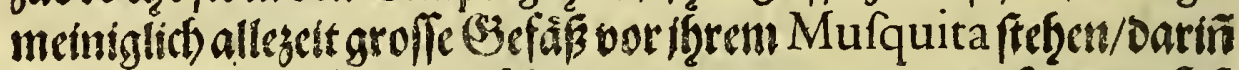

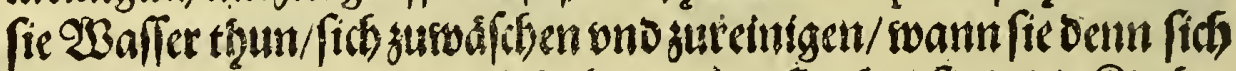

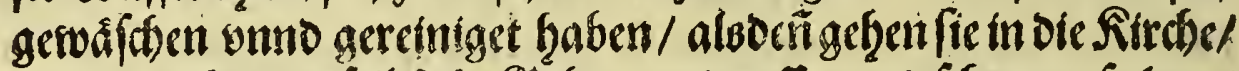

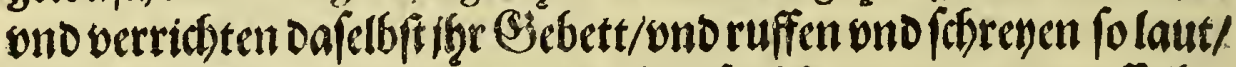

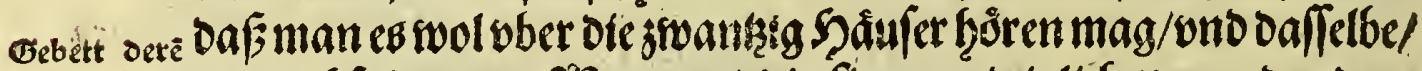
von Banda. mitnath foldenoen 2 borten / oie fie gemeiniglich jwen ober oren imal agen/Stofferolla, Stofferolla, Afcehad an la, Afcehad an 


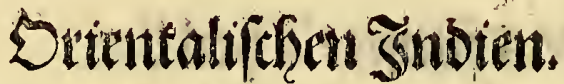

la, Ylla, Afchead an la, Yll lolla, Yll lolla, Machumed die rof-

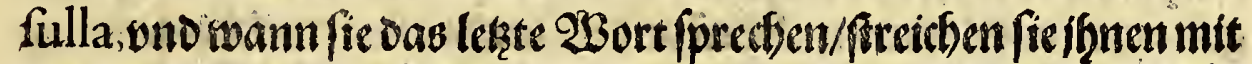

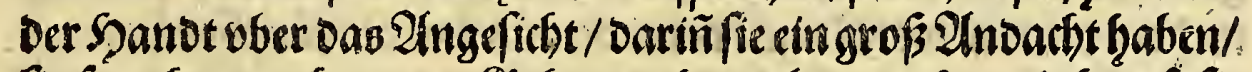
fie forechen auch andere Gebett mebt / aber garbeymlich Dák fie

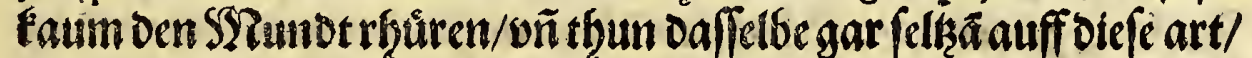

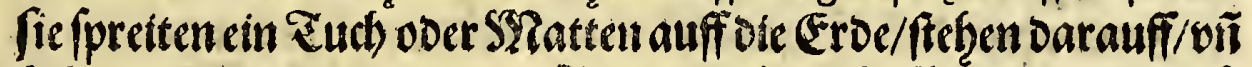

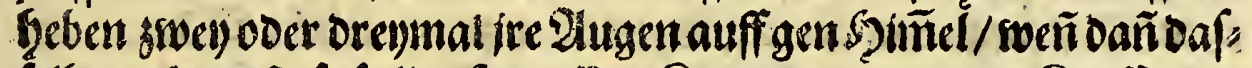
felbe gethantift/ fo fallen fie auffire Sinie/ ond legen oen Riopffiswen.

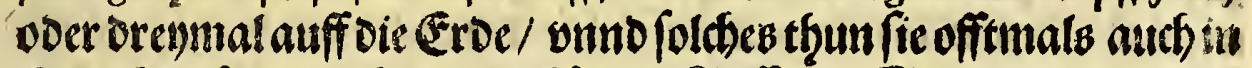

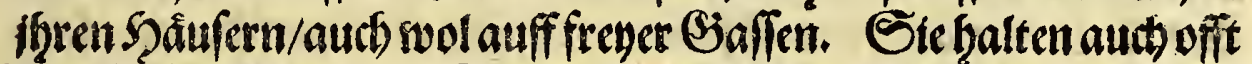

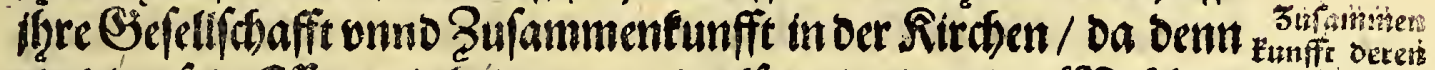
cinjeoerfein Efllen mit bringet / ond alfo mit einanoer SYZafilseit ven Banda. falten / welcher fie audb offtmals in Den walloten jutbun pflegen/ Da fich Dem wol auff oie bundert Perforten bifsweilen verfamb;

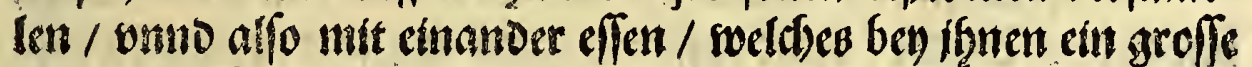

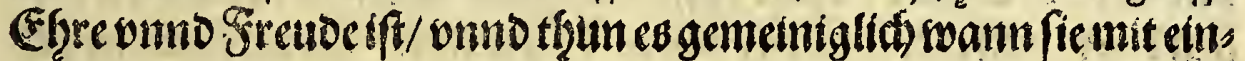
anoer vongeneinen Sanbs Sachen zureden ḩabé. Deñ auffotefer Infel errwan fects ober fieben Stâttlein liegen / soeldhe in fieter

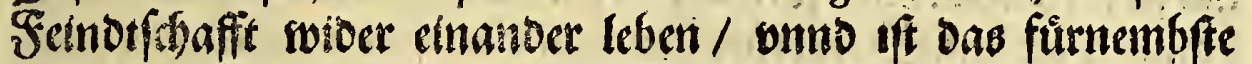

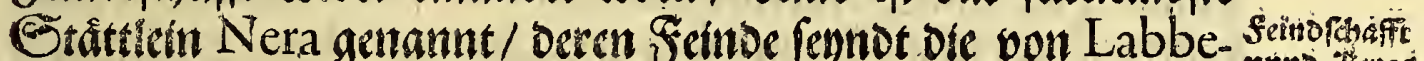

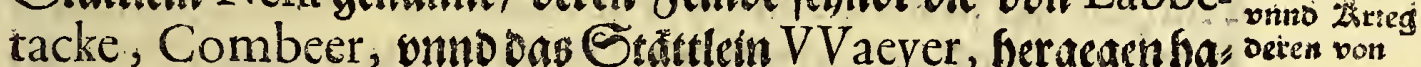

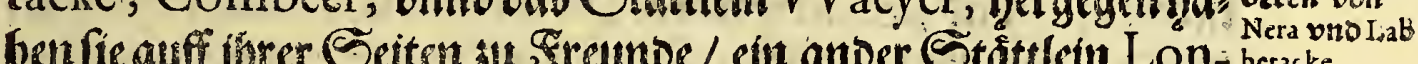

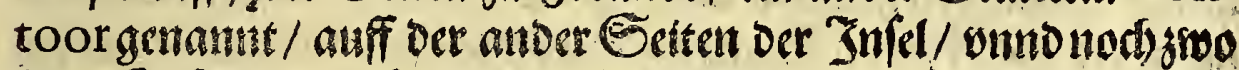

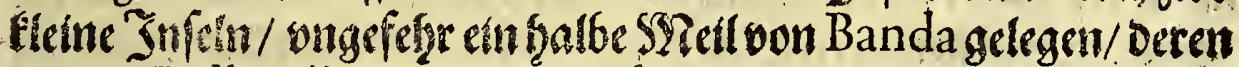
bie cine Polleruiin, Die ander Poelv vay genannt ivtrt: Orefe fennt Greunte beren won Nera, ono soeñ fie woiber oie von Labbetacke

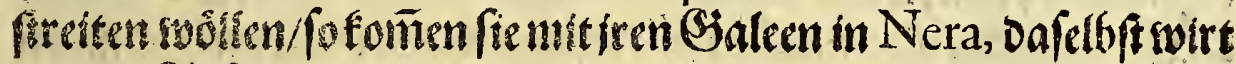

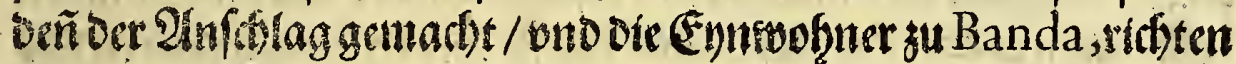

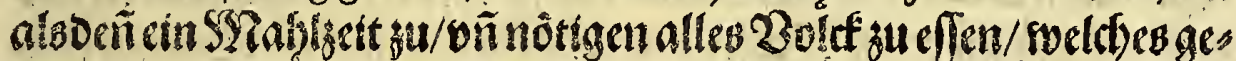

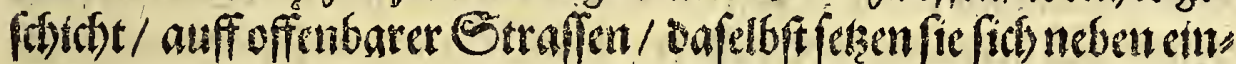

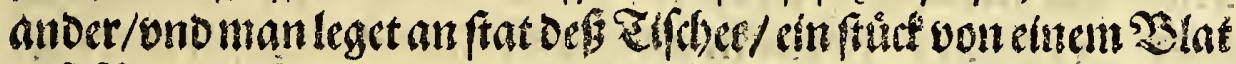

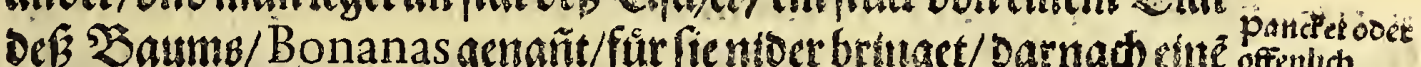

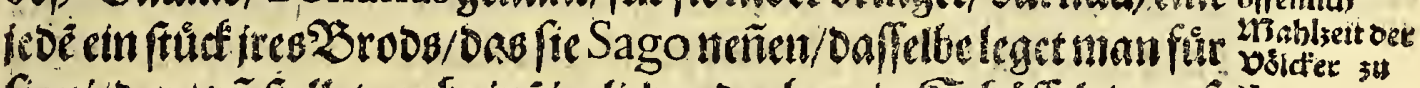

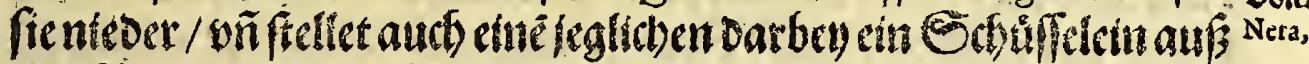

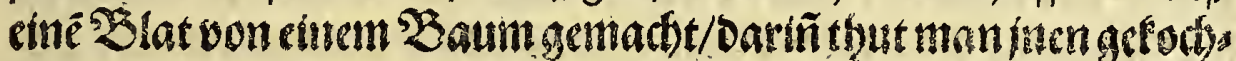

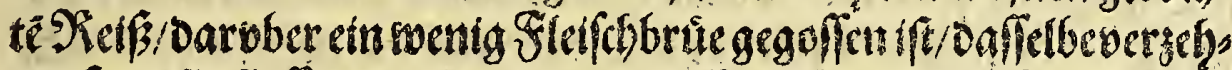

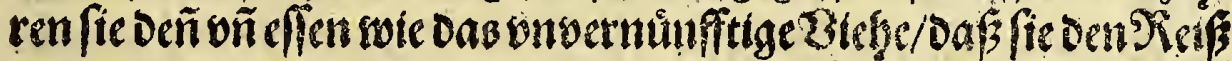

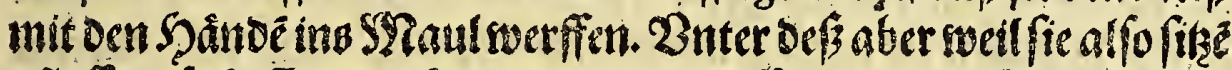

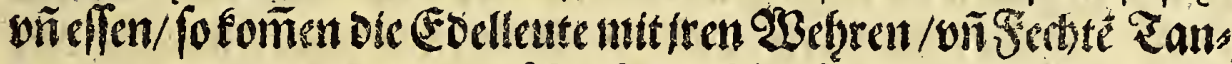

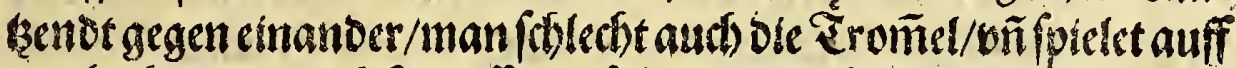

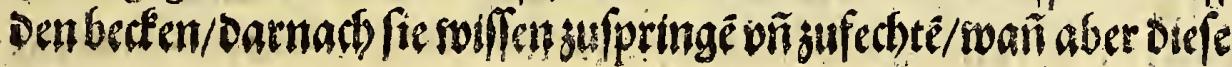
E ij minger 


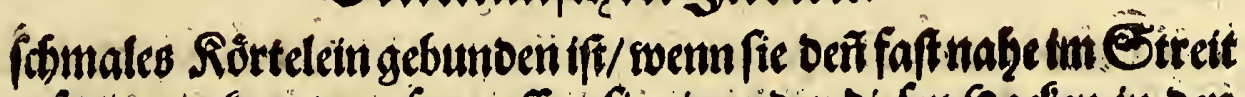
oufanmen fommen/foswerffen /ie einander diefen Sadfen in ben

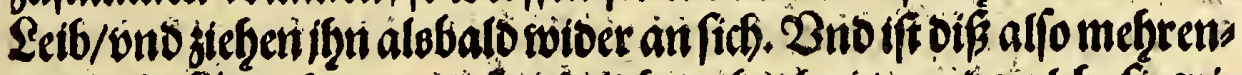

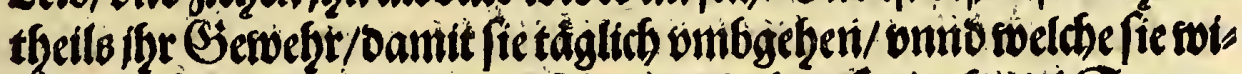

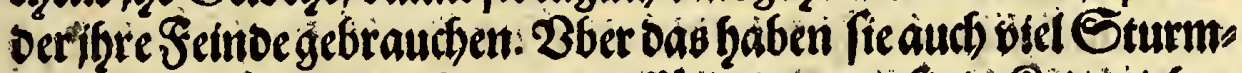

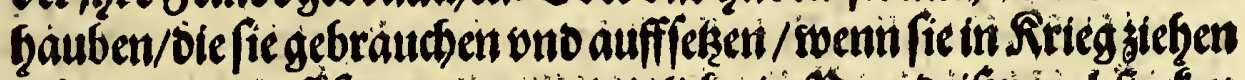

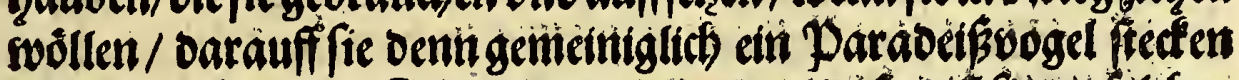

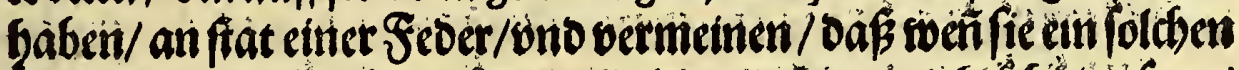

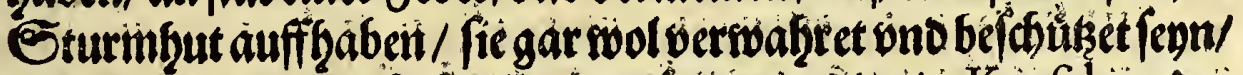
es swerden swar auch Evelleute gefunben / ote èn Korfelet ooer

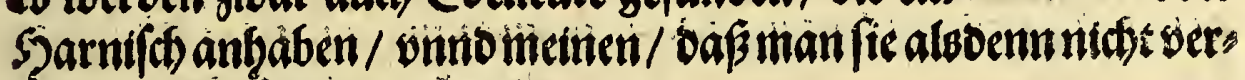
lekzen ober be f

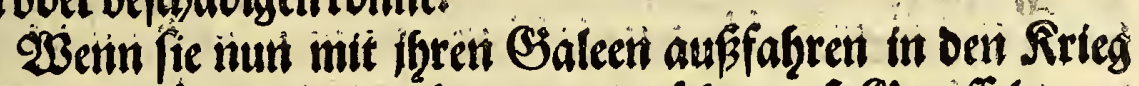

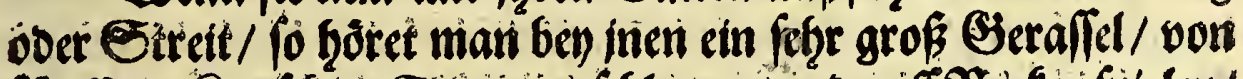

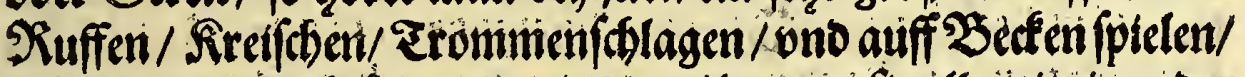

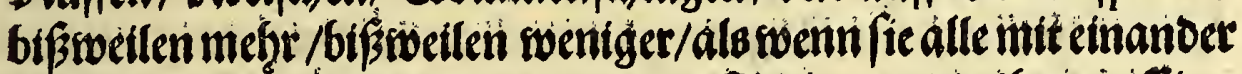

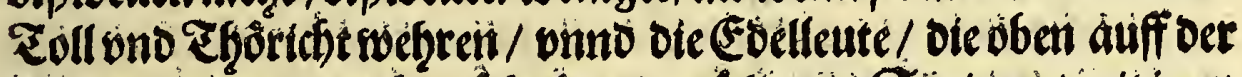

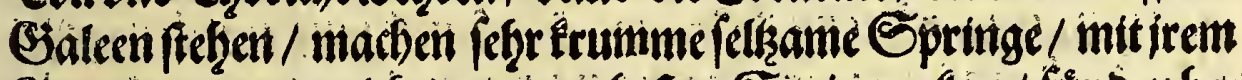

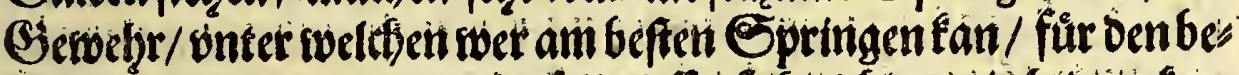

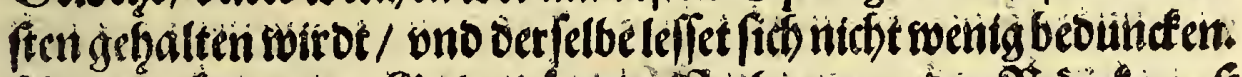

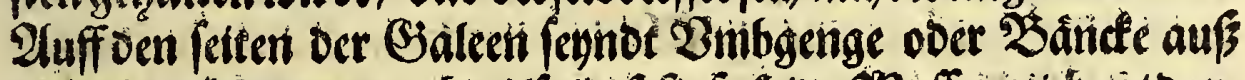

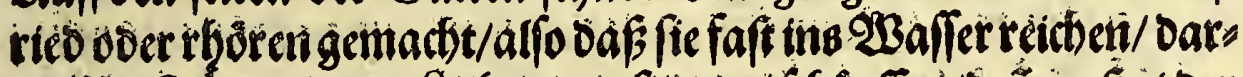

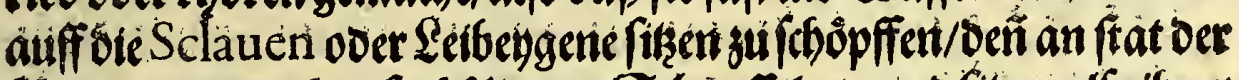

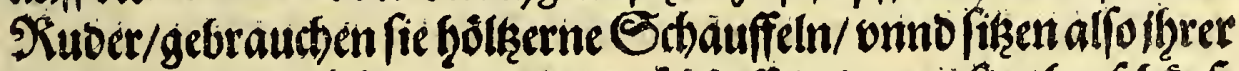

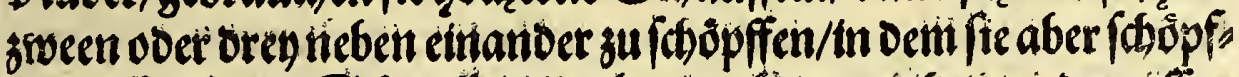

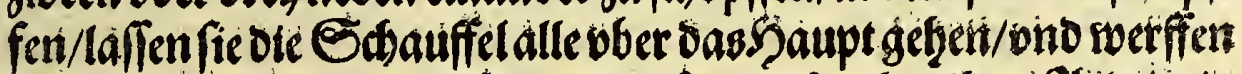
alfo bas 23 afler vber ein feite hinaus / mad)en ben iffer 2 drbeit ein

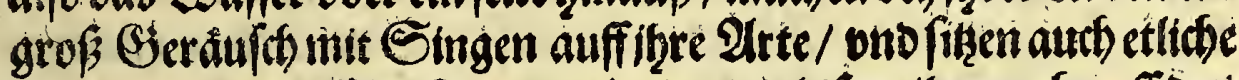
ben ifnen / bie auff Der Erommel / onno ie bifsweilen auth auff bert Becten fétlagen.

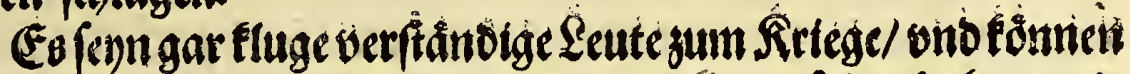
ethatitor oarinn luftig tummeln / wie wir beñgefelgen baben / als bie won Labberacken mit vier Saleen vor bas Etåttlein Nera

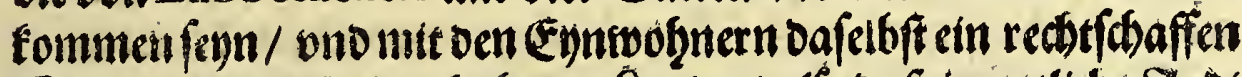

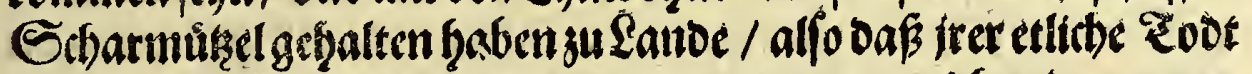
blieben / etliche abor vetel verswundet worden / welches bentenvon Nera vbel veroroffen / oie oerfialben alfo balot fbren 2Inf(d)lagges

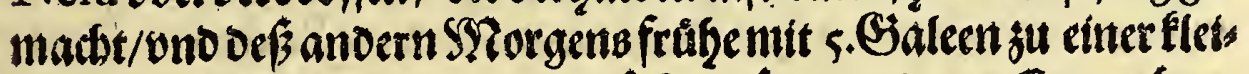
nen Infel V Vayger genannt/gefahren lennot / Deren Ennnoofner Die Nacht vber in oer Statt Labbetacke gerwadhet hatten / fintes mal fie Sreunde mit emanoer fenn/Derbalben als fie be śs STorgens 


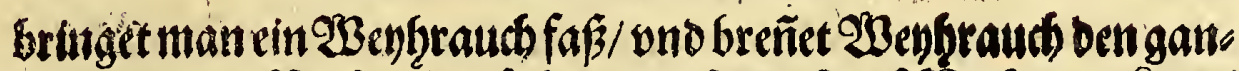

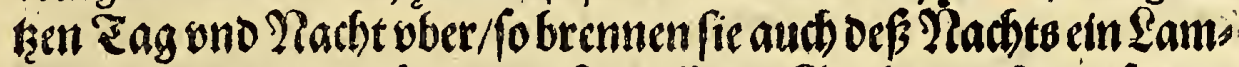

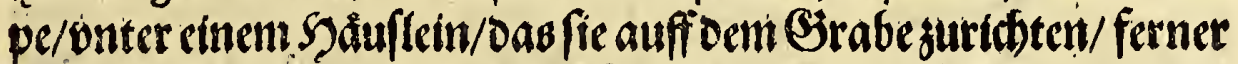

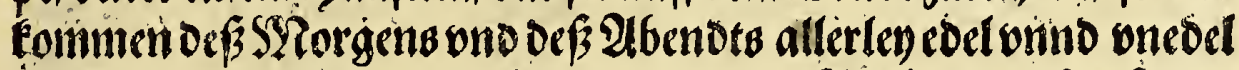
Berfonen / onnd thun ifgr Gebett auffoem Srabe / welthes fie ein lange Zeit al fo halten vno objeruieren. Derfalben wir fie frageten

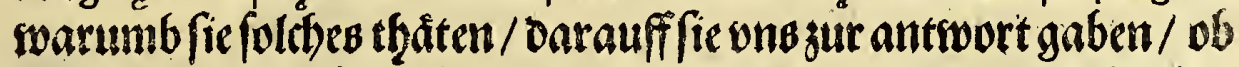

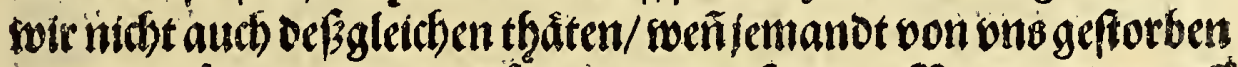

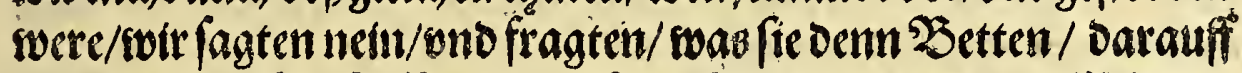

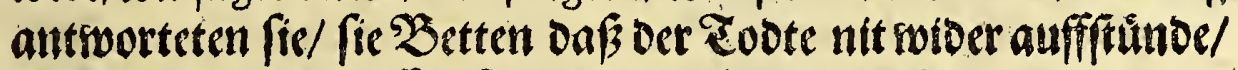

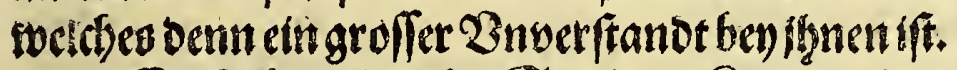

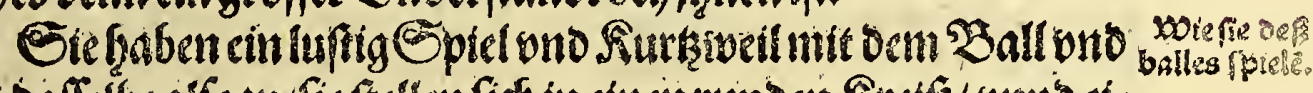

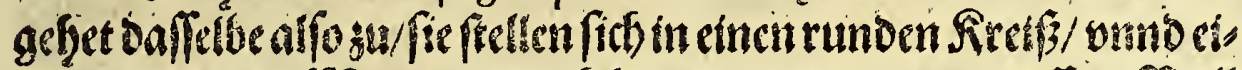

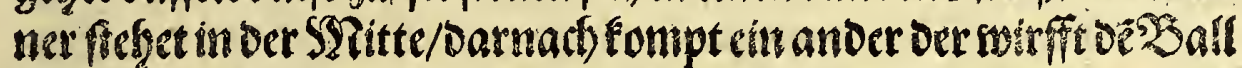

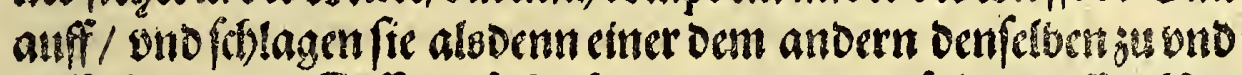

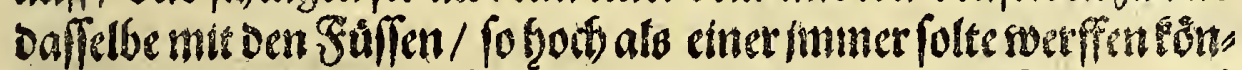

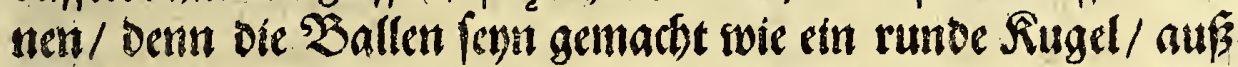

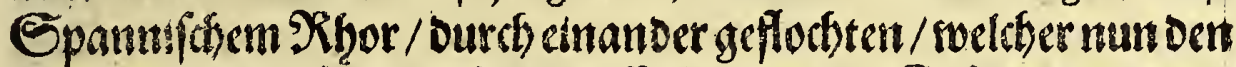

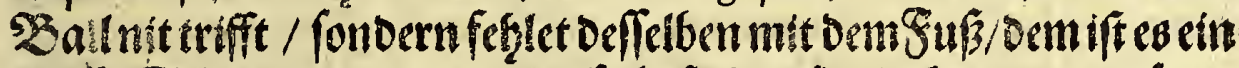

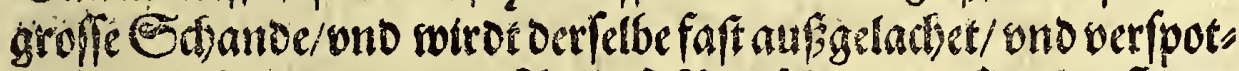

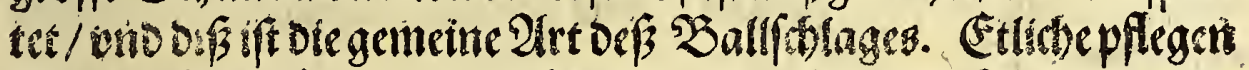

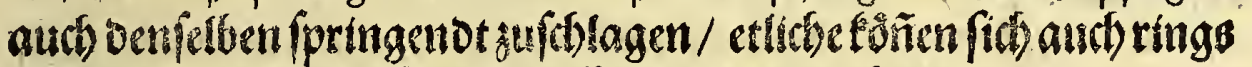

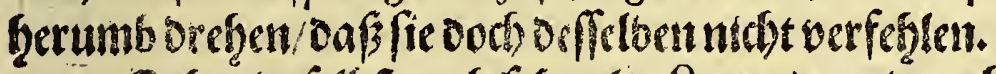

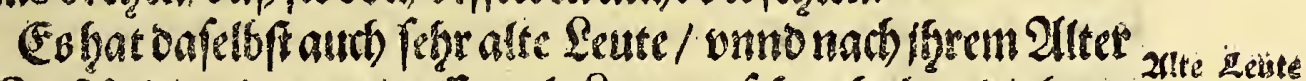

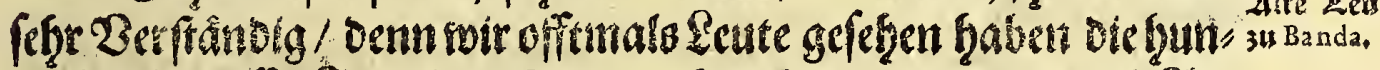

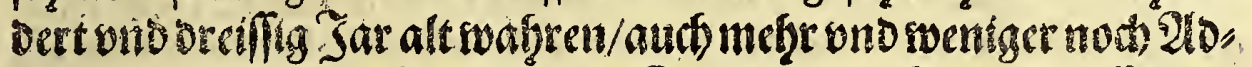

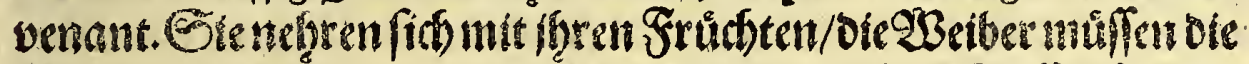

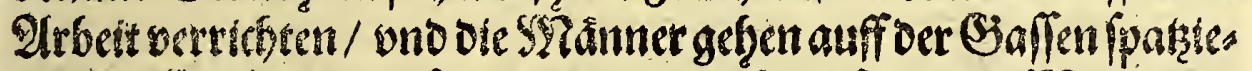

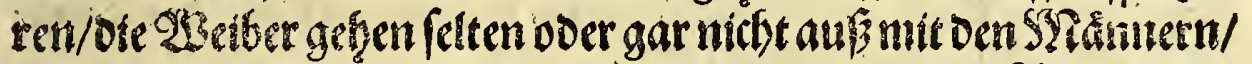

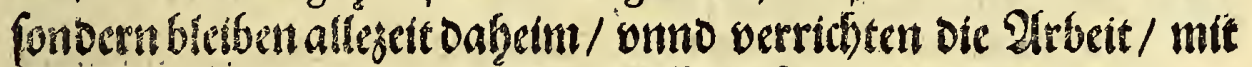

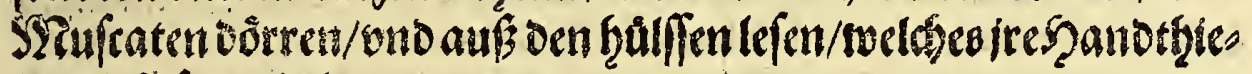
rung iffi/forol als mit andern.

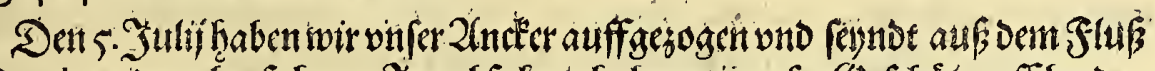

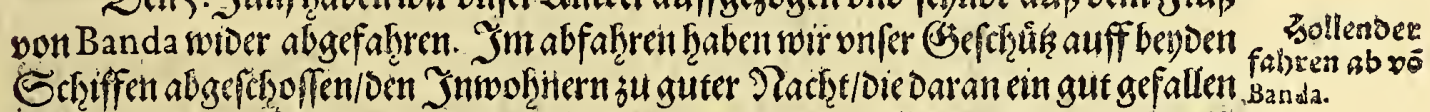

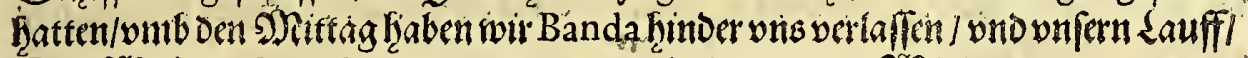

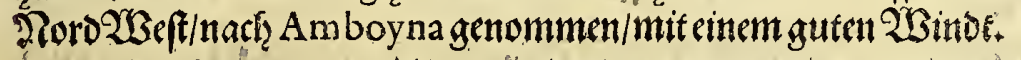

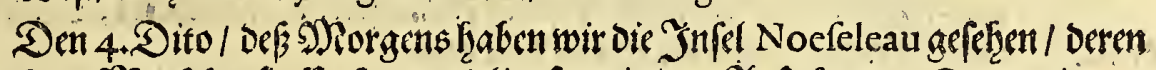

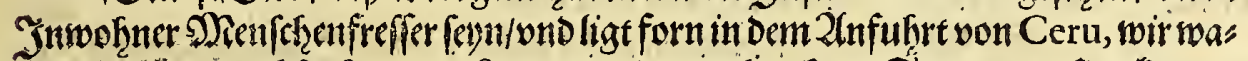
ren aber etrwas jufeher Ferunter fommen / Darumb oak der Etrom gar ftarct warl

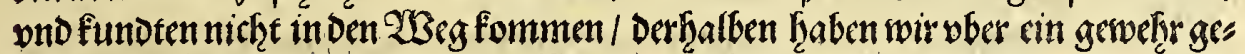

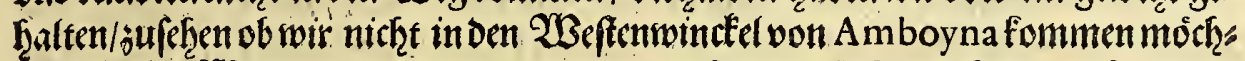

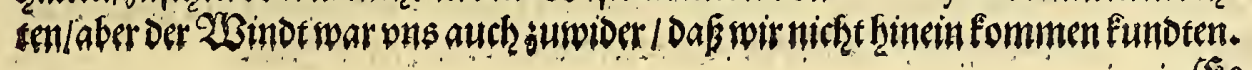

క5 


\section{Erientalifichen Sribien. $\quad 4$}

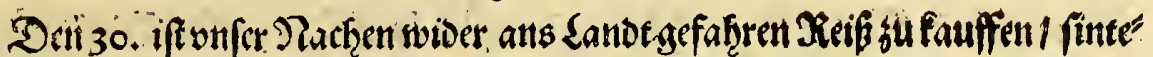

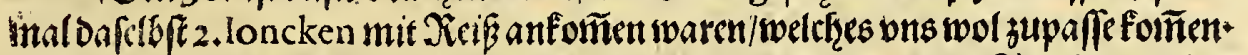

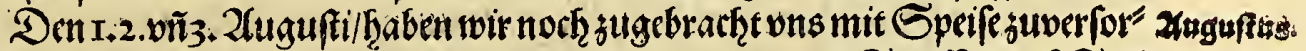

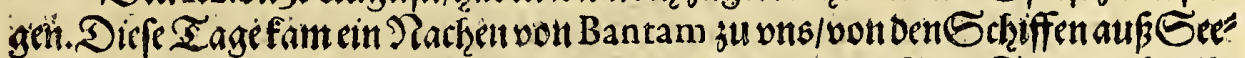

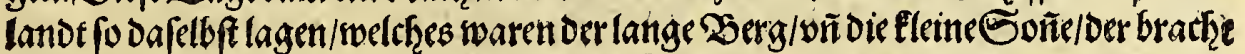

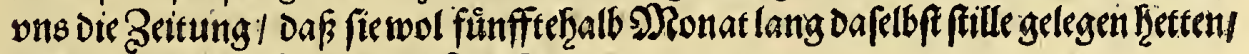
vno warteten auff die neuse Trucht.

Den 4. Dito /.ift Der Bire2lomiral mit Den Rauffleuten von Dem langen Sorg ans Lande gefahren/mit bem Sionig jureden/vnnd ift gemelteb Sthiff gegen Deni Zlbenot/wider waher Bantam gefaheret.

Den s. fenn wirnoch ein mal zu Sande gefahtren/Speik zu fauffen.

Den 6. fentnd wir von laketra abgefafiren/nachiden frifechen Waffer fiuffen/

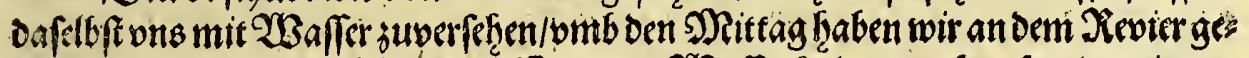

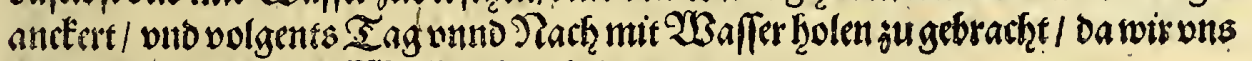

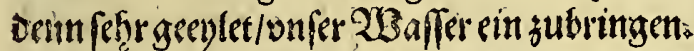

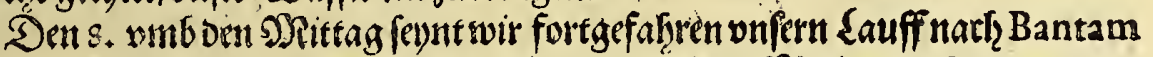

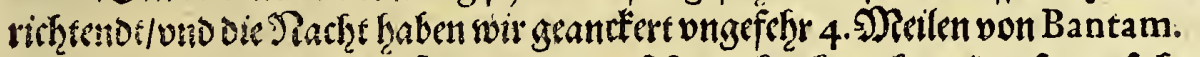

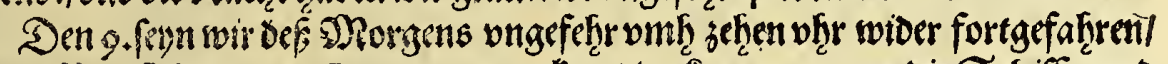

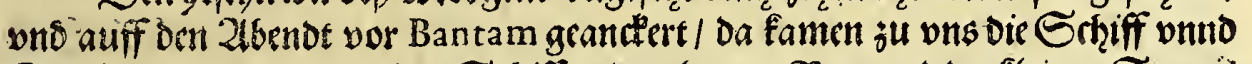

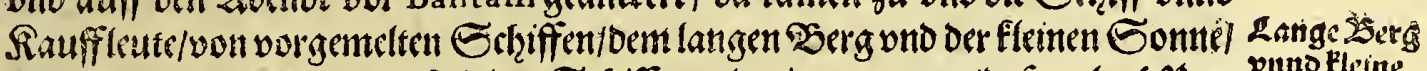

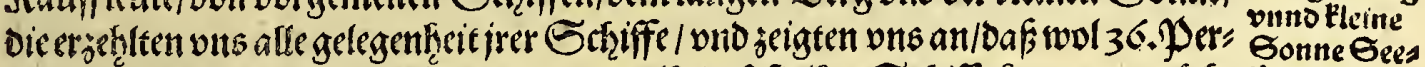

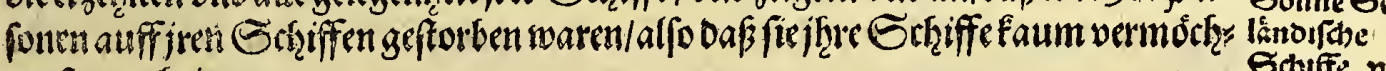
tenf fort outsingen.

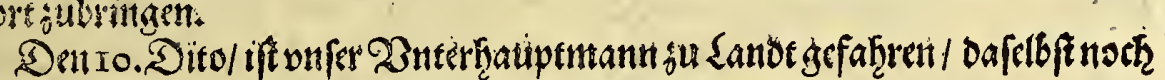
Gdiffe vor: ctfiche Portz feleinen vnd andere Sachę

Den 14 . ift ju Mittage der Statthalter von Bantam, wod mit vier ḩunbere

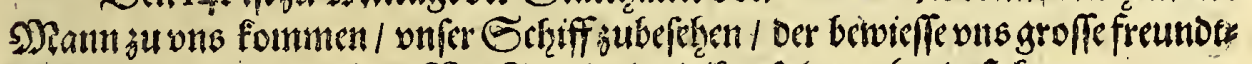

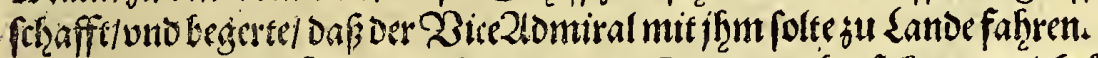

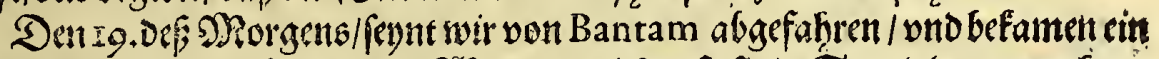

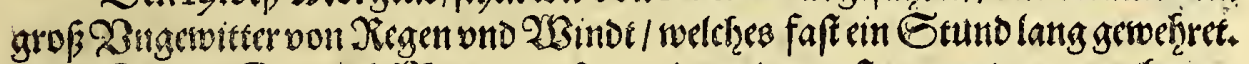

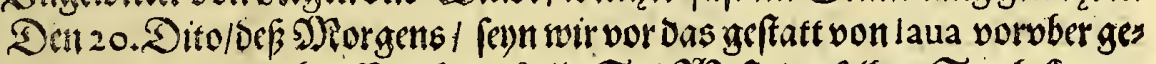

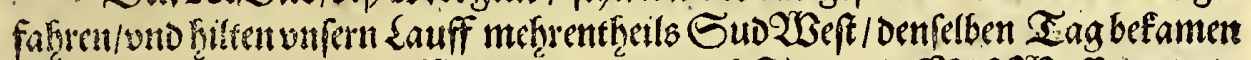

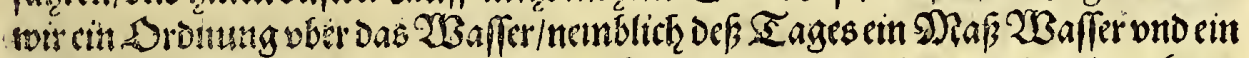

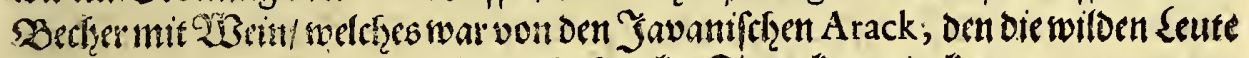

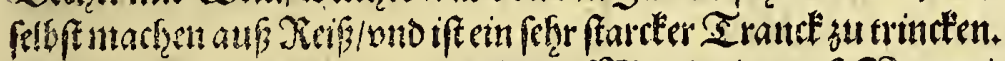

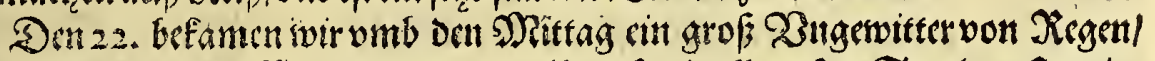

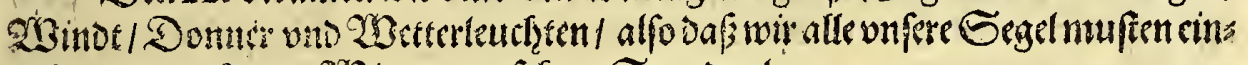

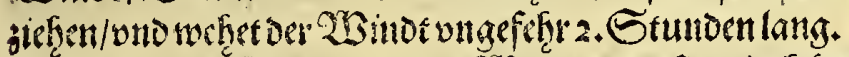

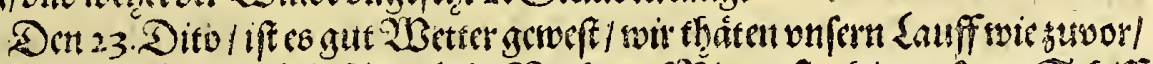

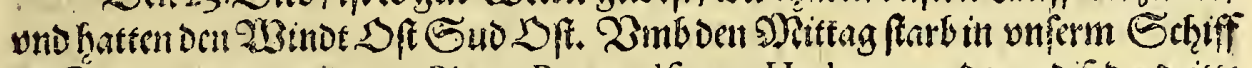

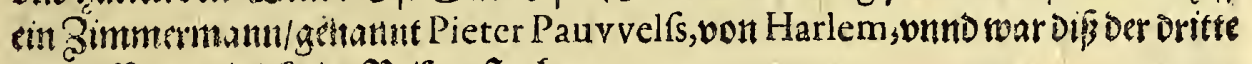
Der auffocr miderheim Reifegeftorben:

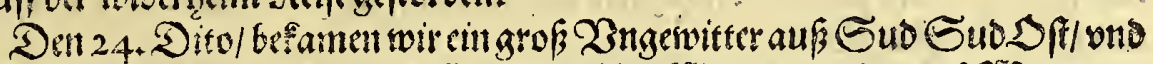

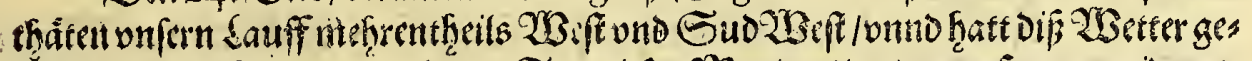
withret bif auffoen 3r.oder lezgen Tag dicfes Dionden/ daes angefangen wider ets souseffer zumeroen.

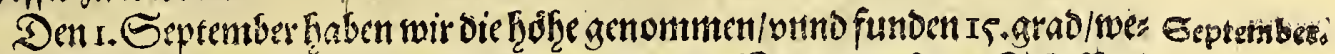

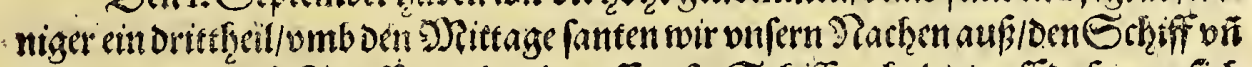

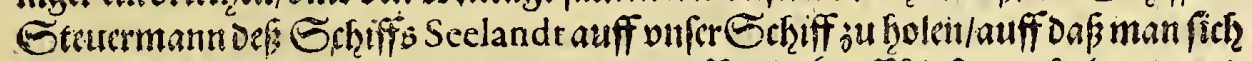

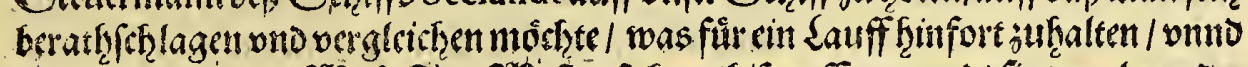

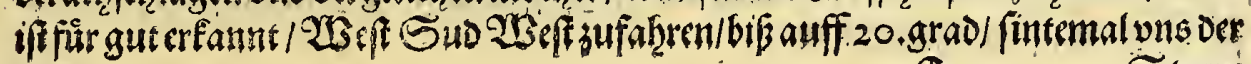
J Etrom 


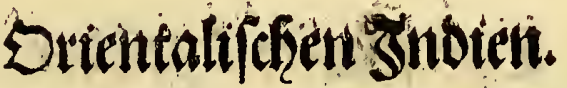

Wind.

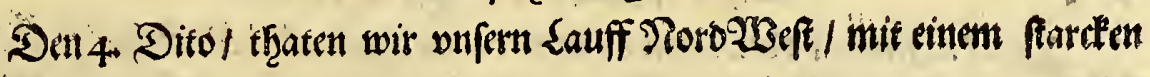

Den 6. Dito/haben wir auff onferm Schiff ein Turteltäublem gefangèt!

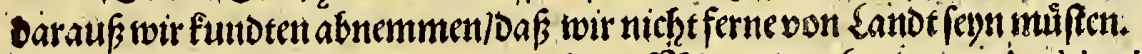

Den ro. Dito / fraben wir vmb den Mittag das Lande von Aethiopia gefes

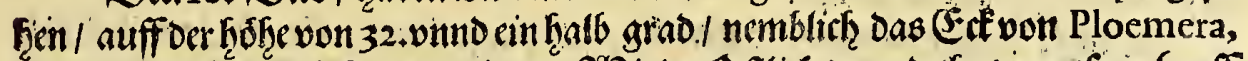
gegen brm 2 benot befamen wir ben 2 Binot Sftlich/ bund thaten onjern Lauff EnDTBeft:

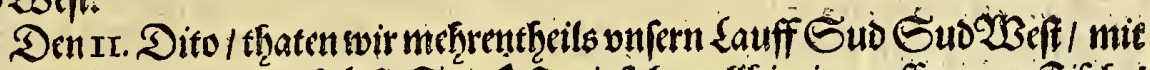

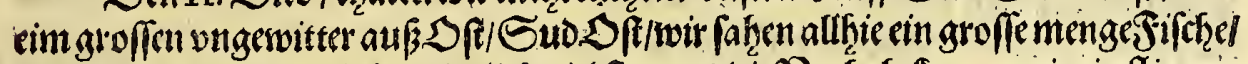

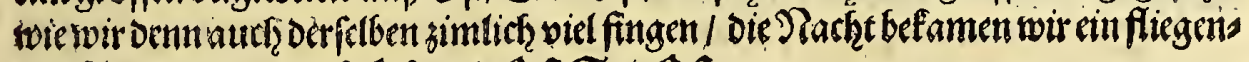

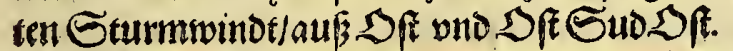

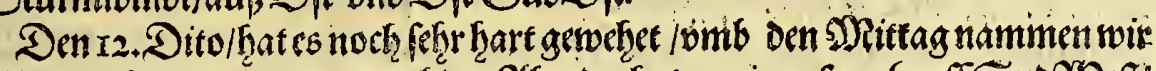

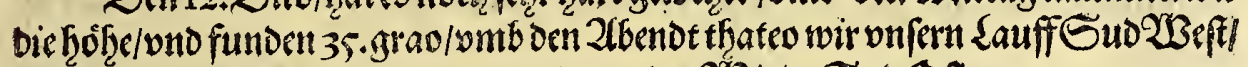

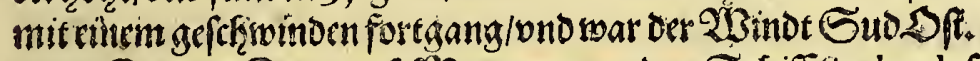

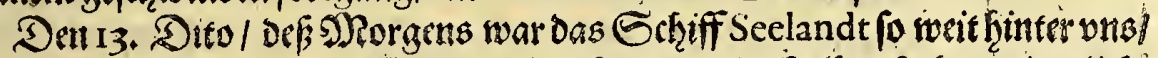

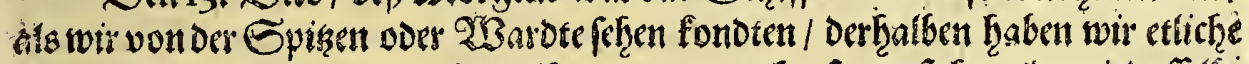

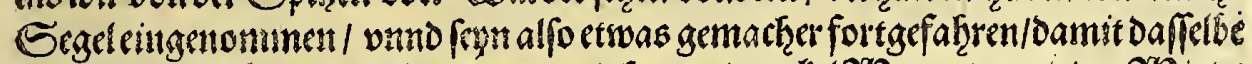

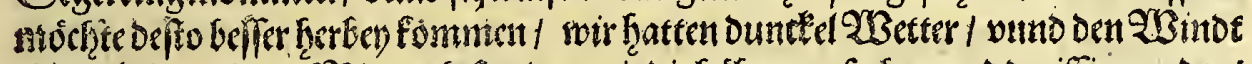

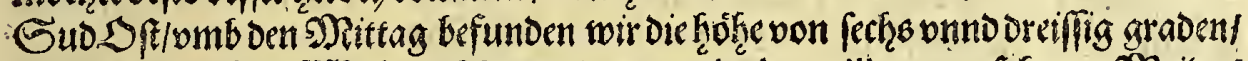

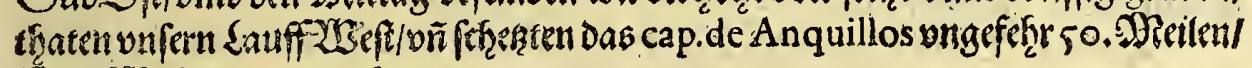

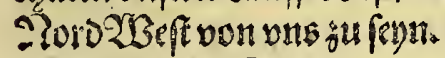

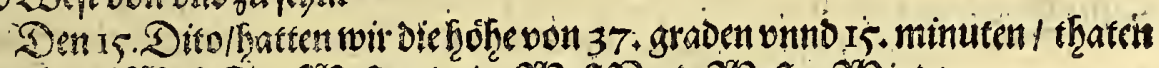

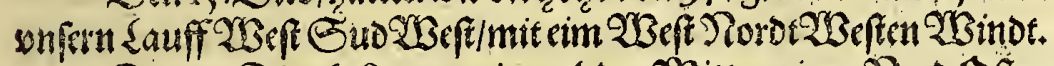

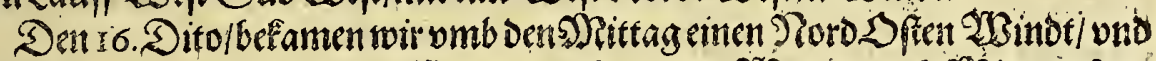

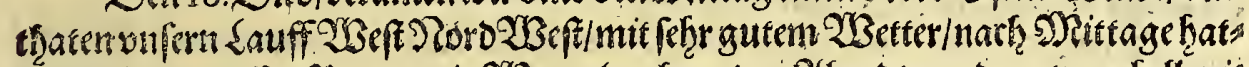

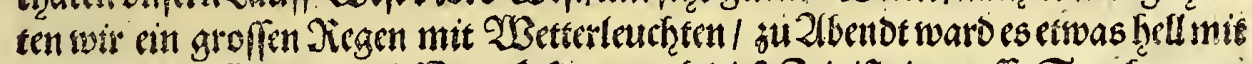

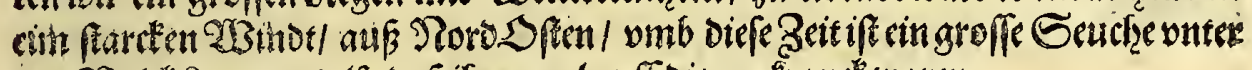

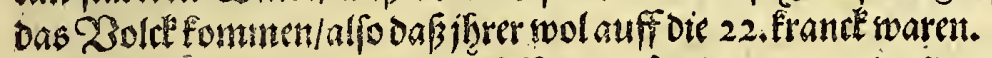

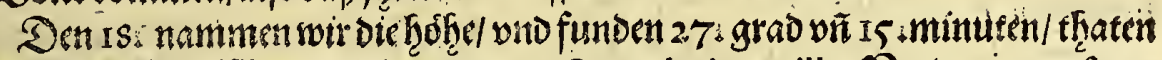

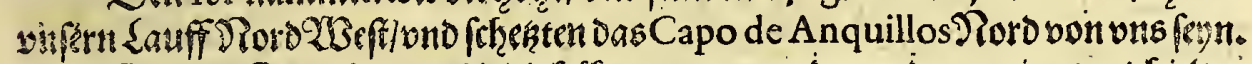

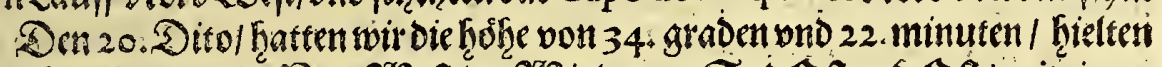

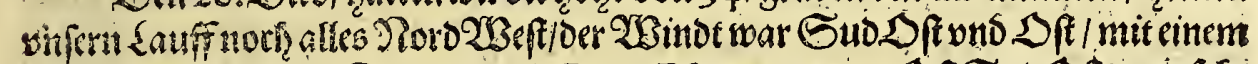

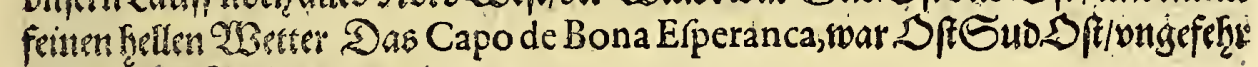
anffoic rs. SRethen von vits.

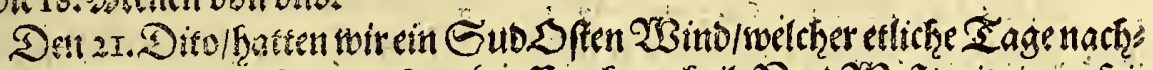

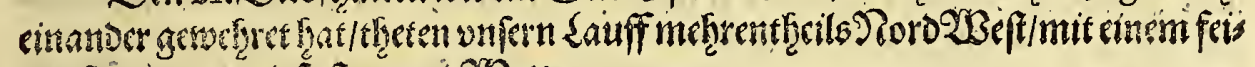

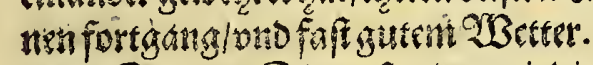

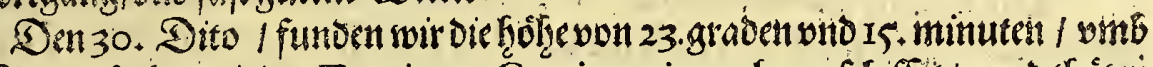

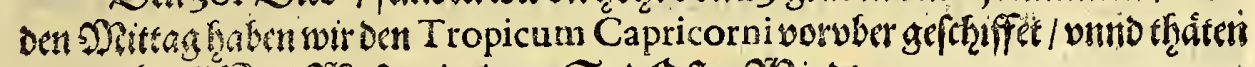

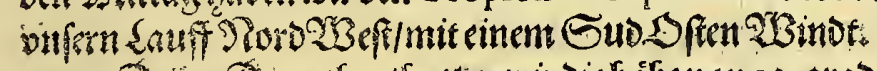

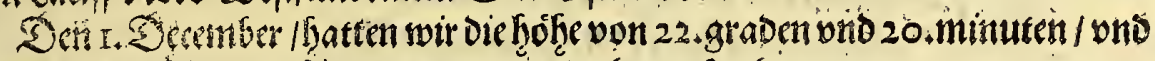
befantentiefent Tagdic Eonne wiber gerad ober bufer Saupt.

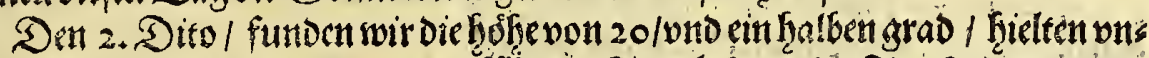

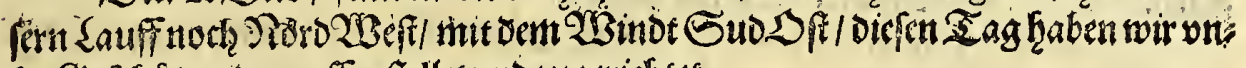
for Grechich mider auff geftetlet wnd jugerichetet.

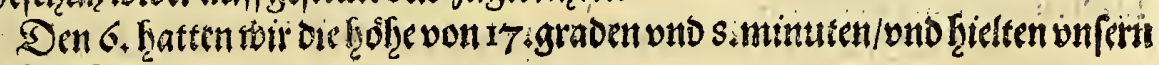
altêt Sauff.

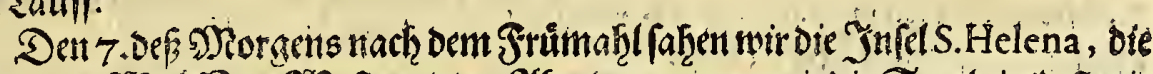

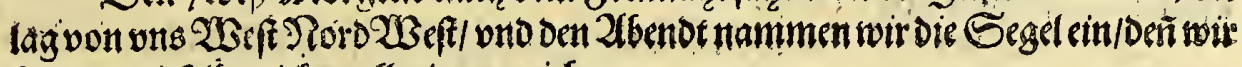
fondeten diefelbe nicht toollendes erreichen.

Fij Den 


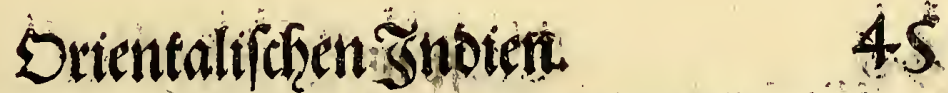

Den 26. funden wir bie hrofhe von 28. graden 1 thaden onfern Sauff Sord

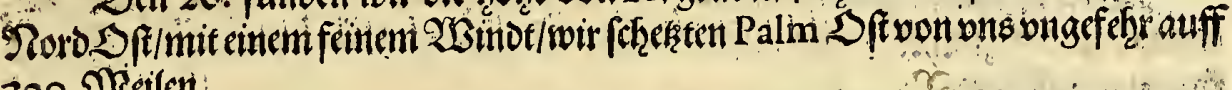
300. Sicilen:.

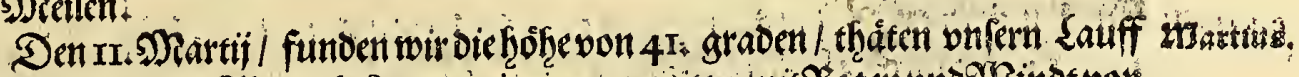

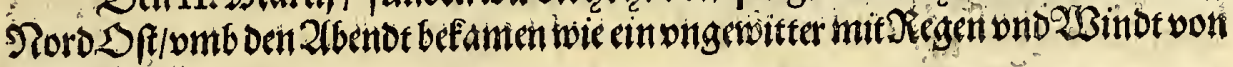

Noro Siteri.

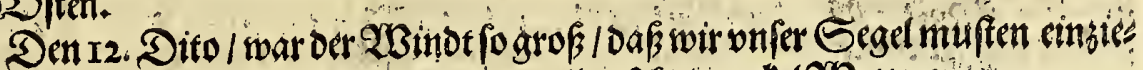

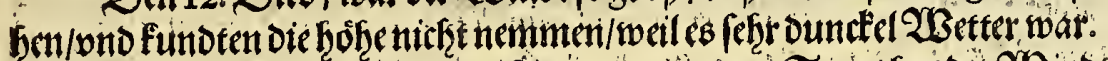

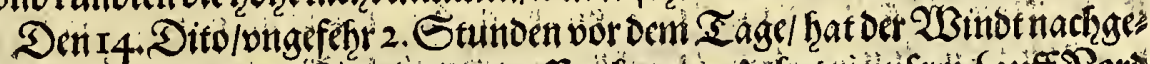

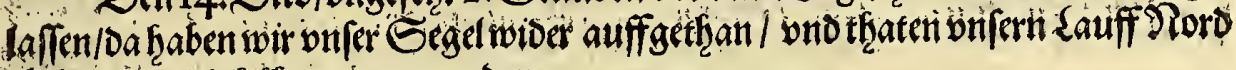
SSit/hattendie höhe von 42 graden. Sfen.

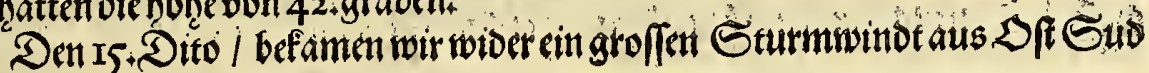

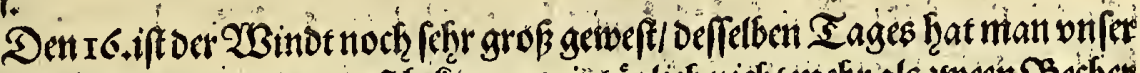

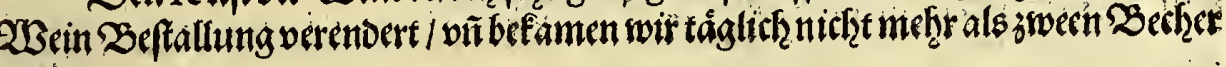
woll.

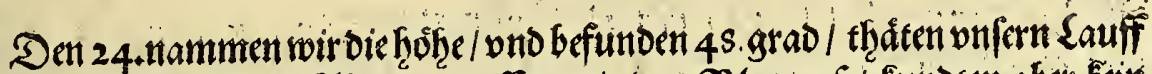

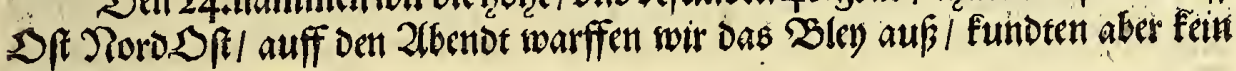
Grumbt finden.

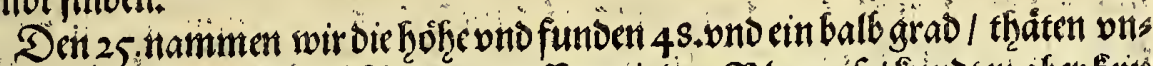

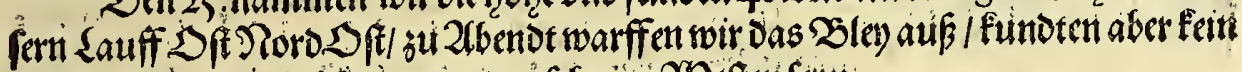

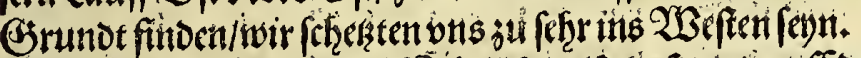

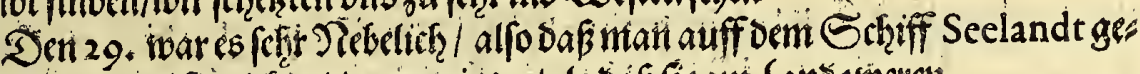

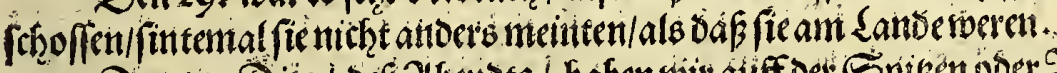

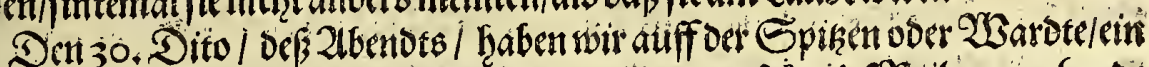

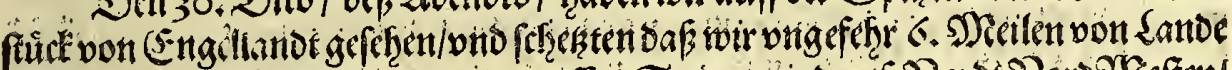

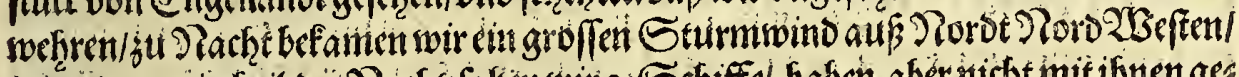

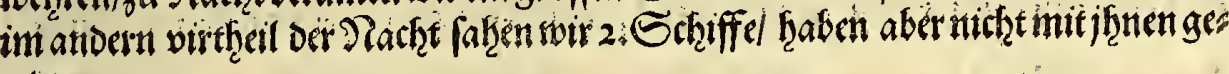
rcot.

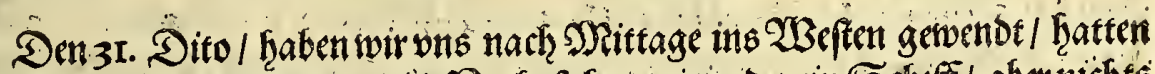

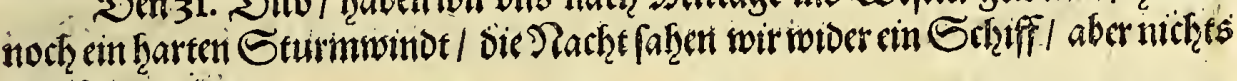
mitjfim gercot.

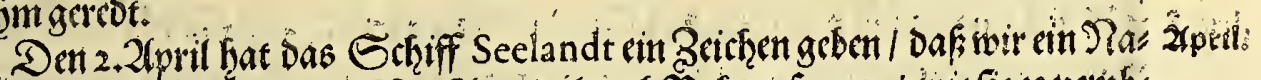

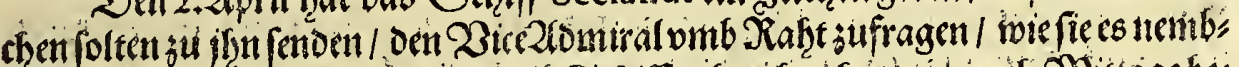

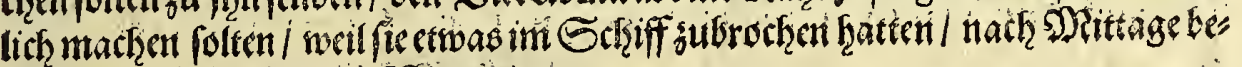
famentwir rbibertimb cin Wrigetsitter.

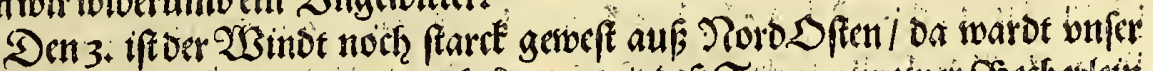

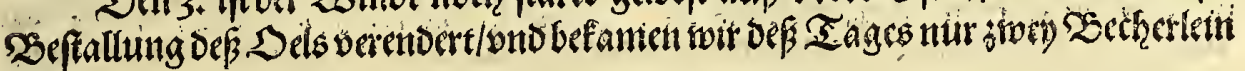
woll.

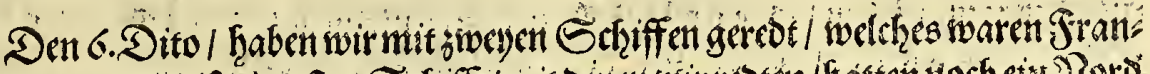

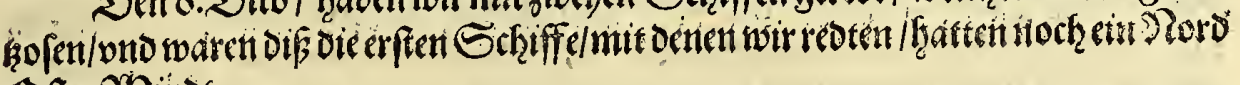
Dfen2Binot:

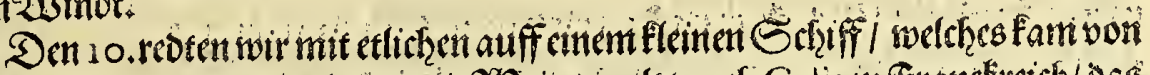

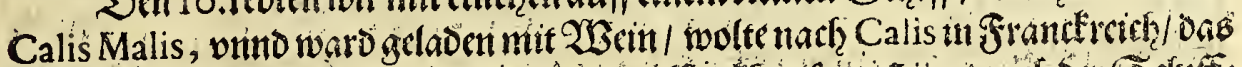

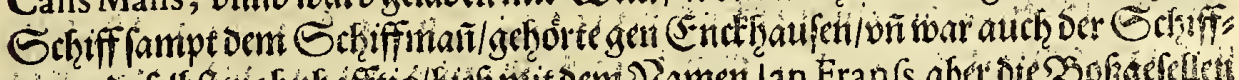

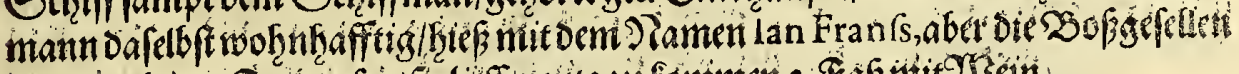

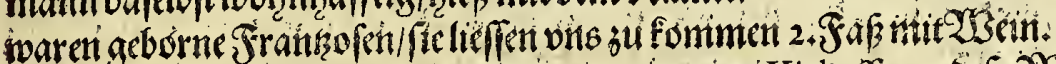

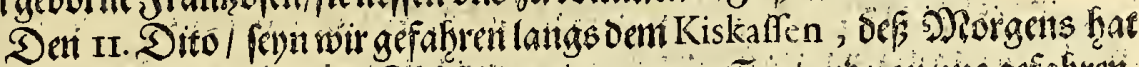

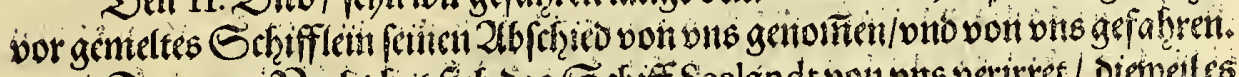

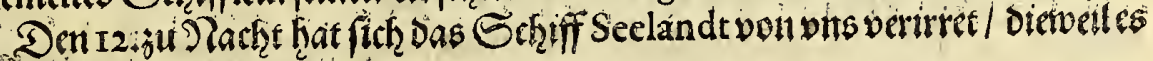
fort Simfer ond Pebelich war

iij Deti 


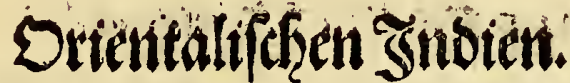

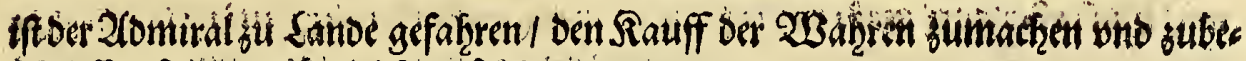

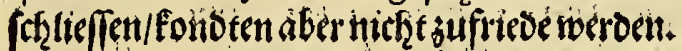

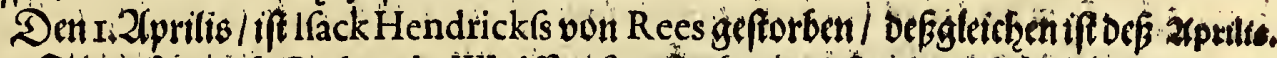

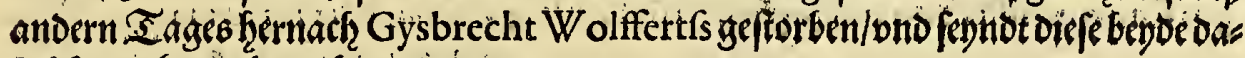
felbft ant Sande begrabentworben.

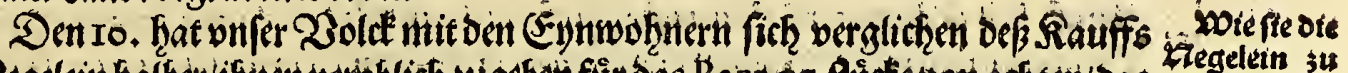

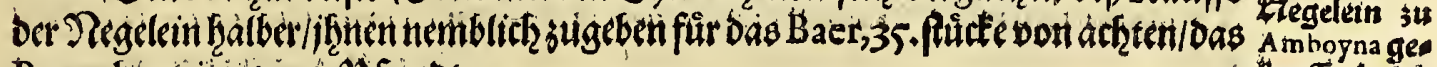
Baer aber iviegget $55^{\circ}$. Pf fundt.

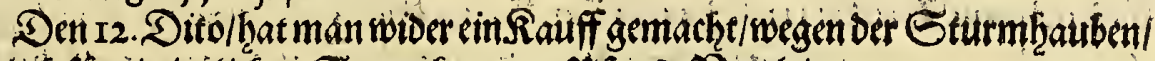

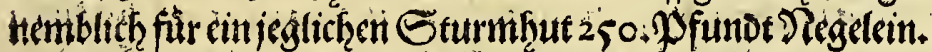

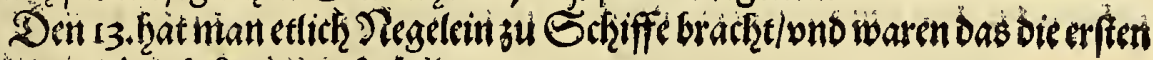

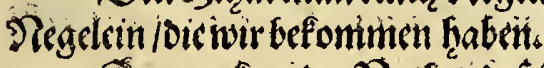

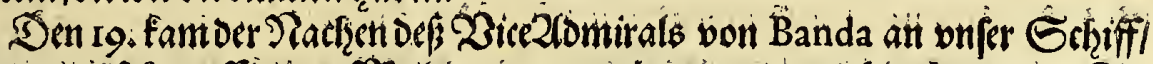

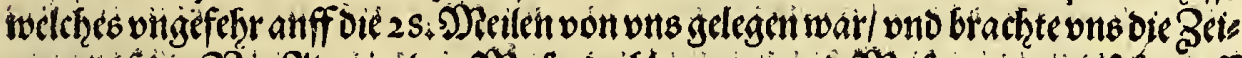

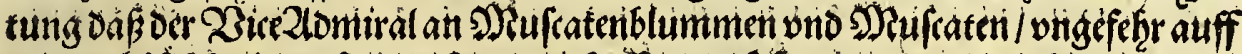

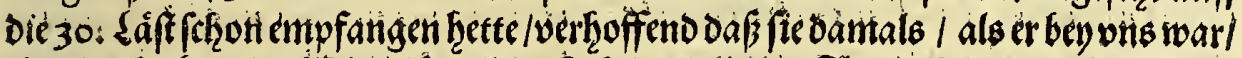

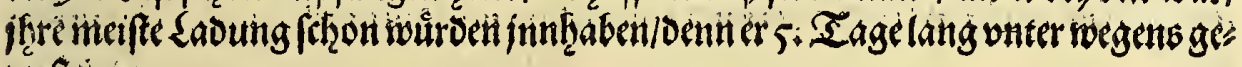
weeftwar.

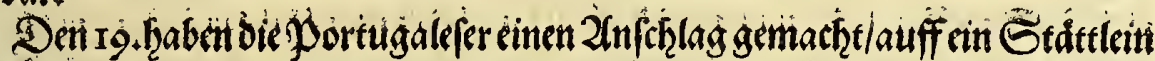

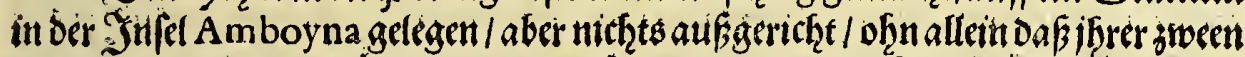

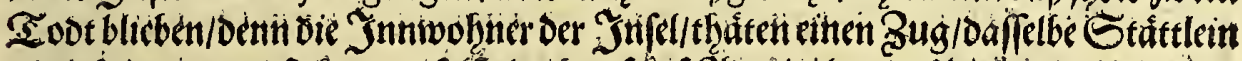

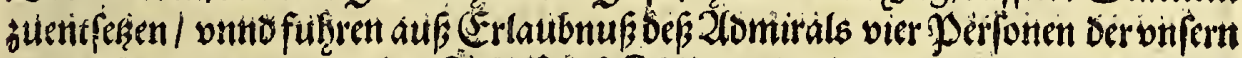
mit in Dem Prauvven oder Echiff Def Ronitgs von Tetnate Bruder / welches

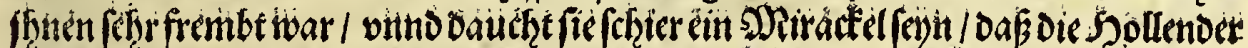

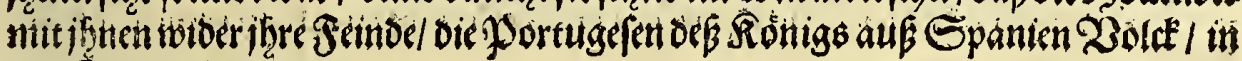
Den Srieg zietien fölten.

\section{cin 2 belster.}

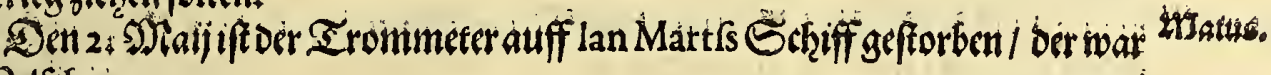

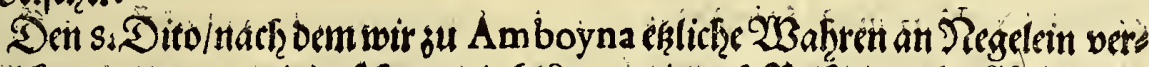

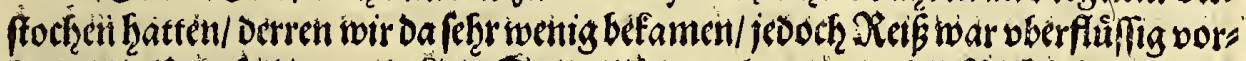

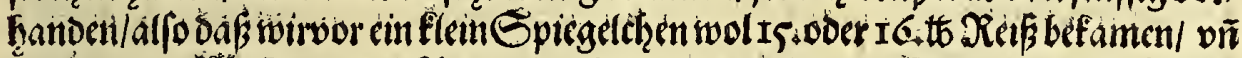

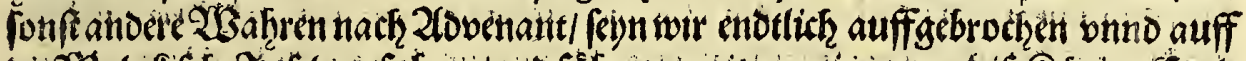

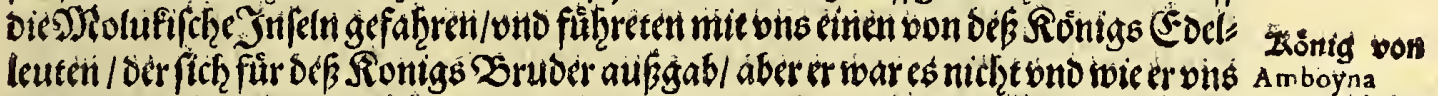

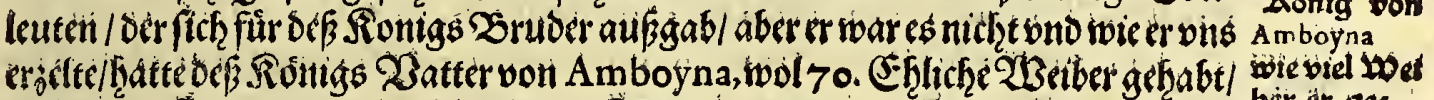

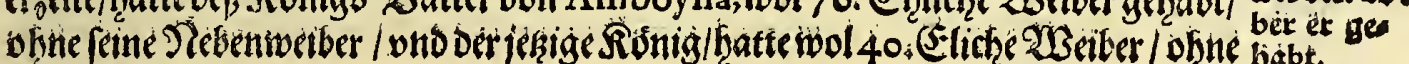
bie Nebentweiber.

Den r 4 . Sito/ift Albert Pitertss geftorben auffoent كithifflan Martss.

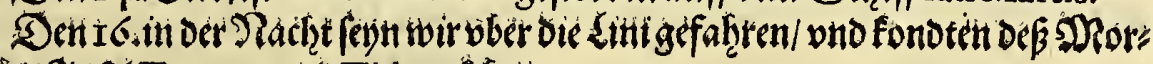
getió bie Infel Ternate wins Tidere fotheti.

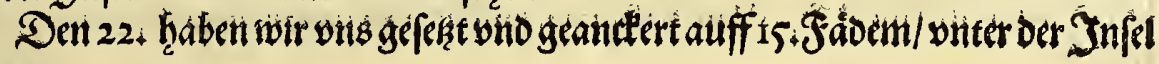
Ternate.

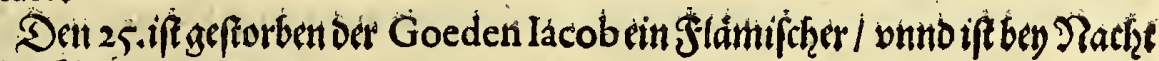
auffoèr Ynjel begraúet.

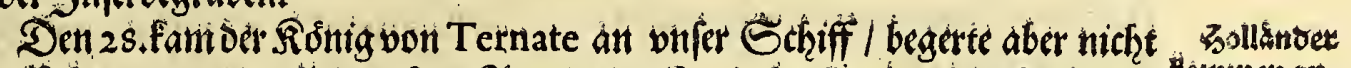

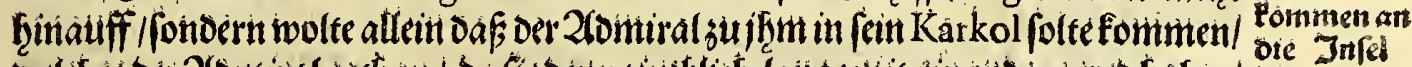

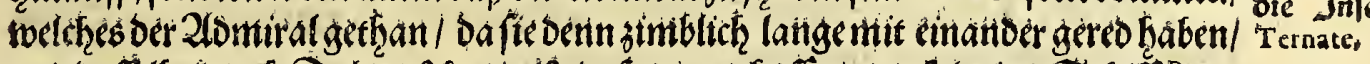

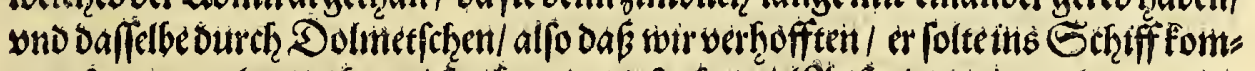

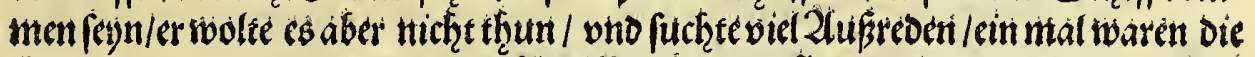

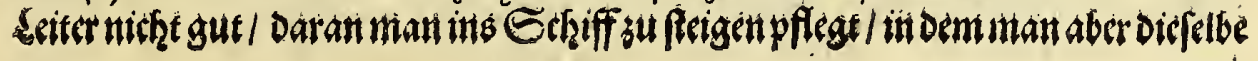

thite 


\section{Grientaliferen \$nbien.}

Siónig fant untb oen 2 bendans Schiff / aber er woltenicht hintein fommen / fon

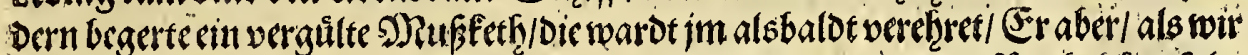

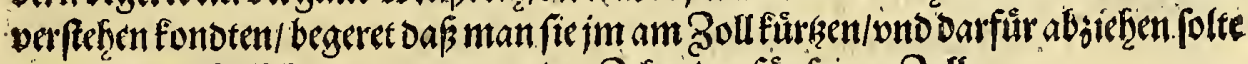

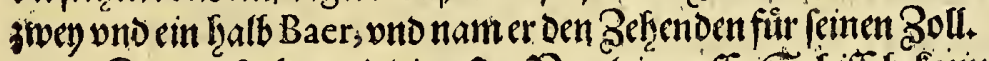

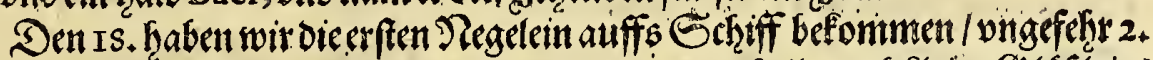

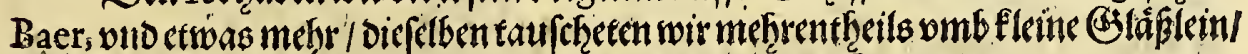
wed die Etorger brauchen/ond gaben 60 für in Baer ?tegelein.

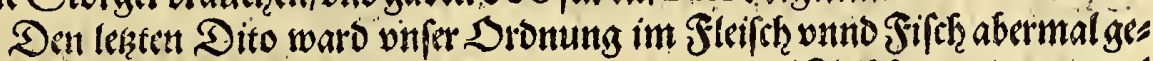

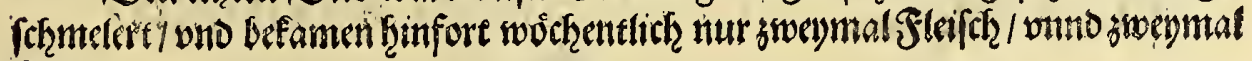
Sifitre.

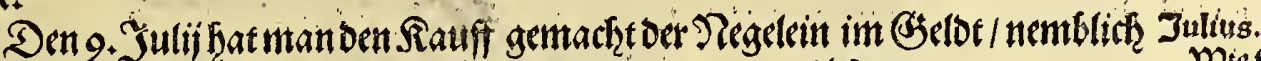

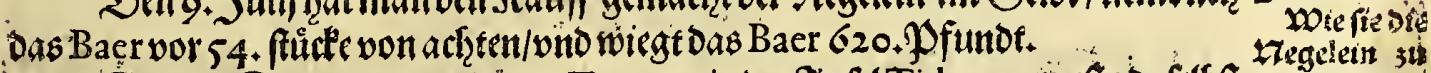

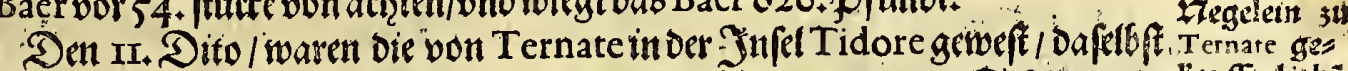

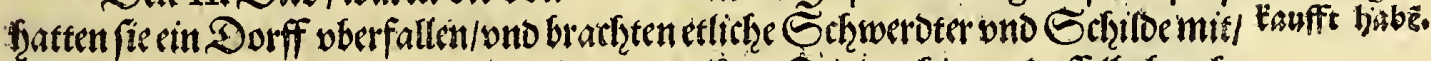

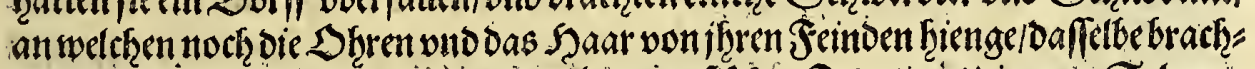

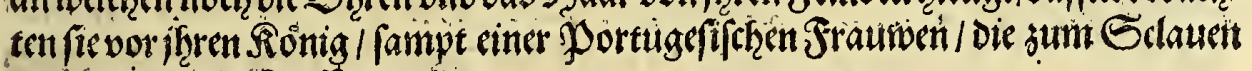
gentach tono verfautfet warbt.

Den 24. Dito/waren Die von Ternate, twiber in ber Injel Tidore getweft! ono brach ten 5. Shá

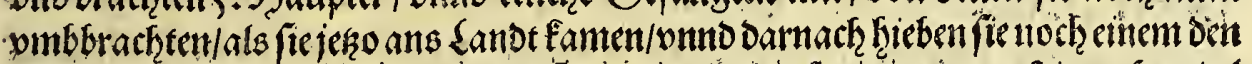

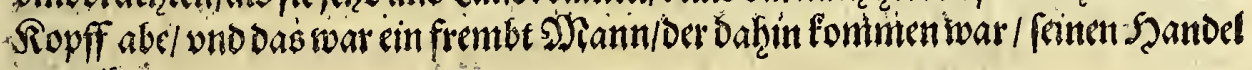
outreiber.

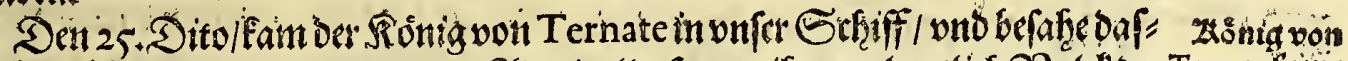

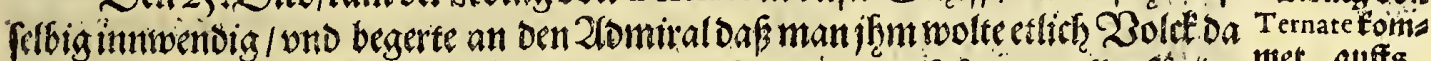

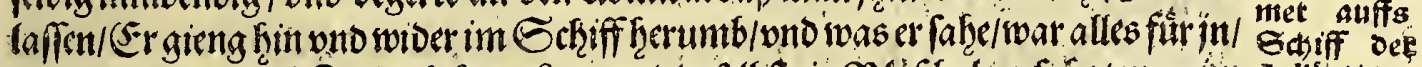

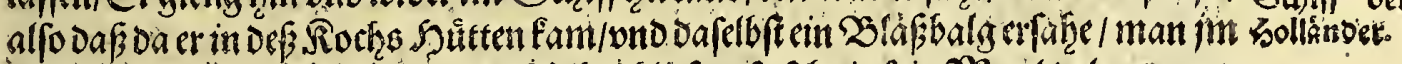

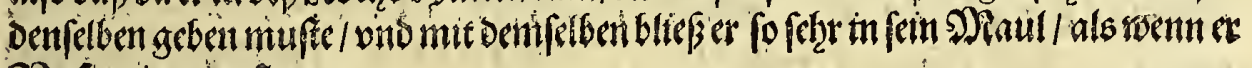
Znitutigg getweftwor.

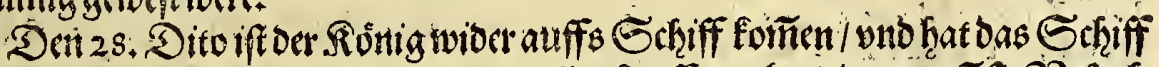

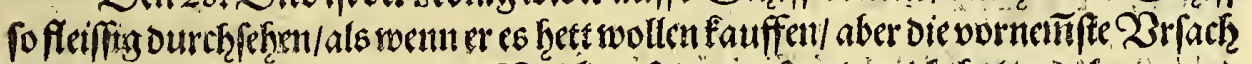

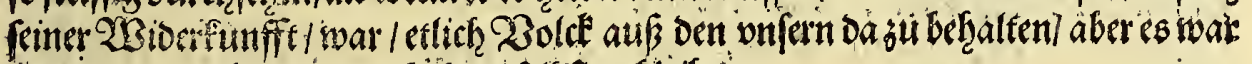

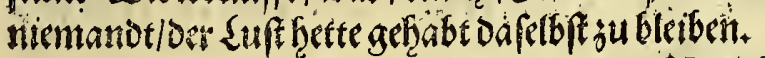

Den 29. Dito fant Dafin ein Prau aut Banda, bno bractęe trits Beiturig won

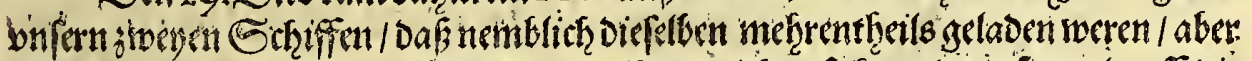

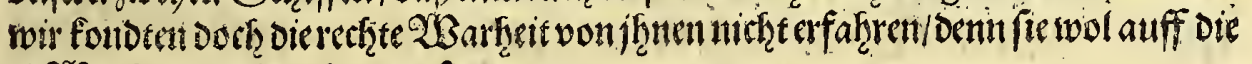
$6,2 B$ oflen won Banda gerweft waten.

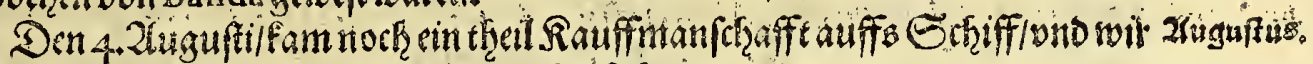

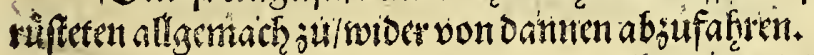

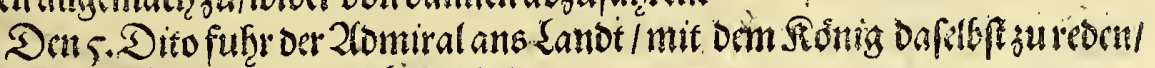
toelcterermit frinon Karkol ant Sando lagt.

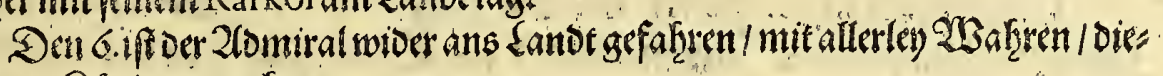
pelbedem Rontig gubcrefien.

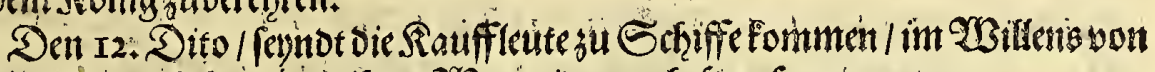

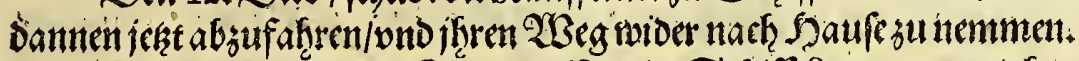

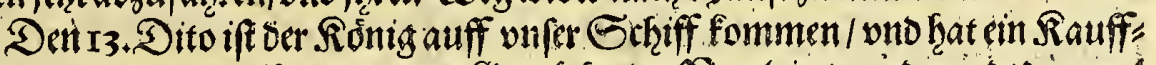

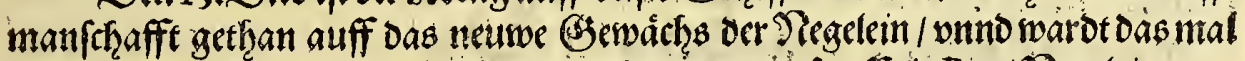

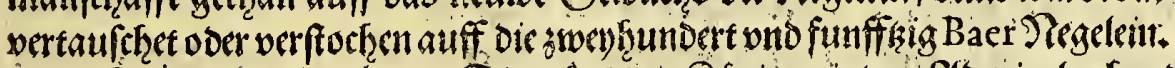

Den 14. I5. vnno I6. Dito/hat Det Rontg mit Dem Zlomital geḩandelt !

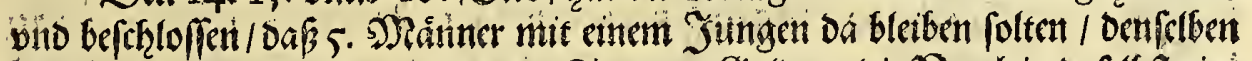

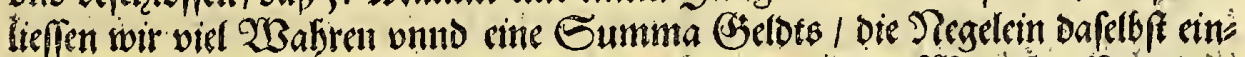

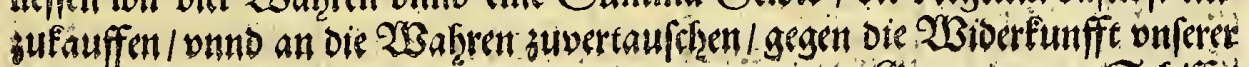




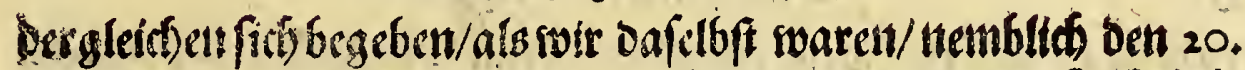
Suli 2m. 1599 . Deñ die von Ternate fufren aufi gemelte In/el Ti-

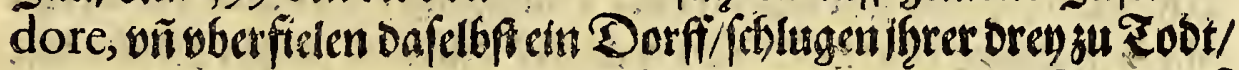

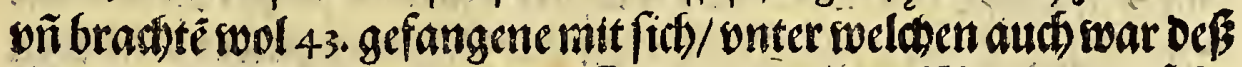

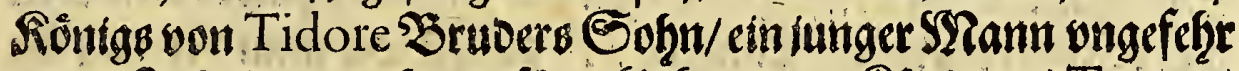

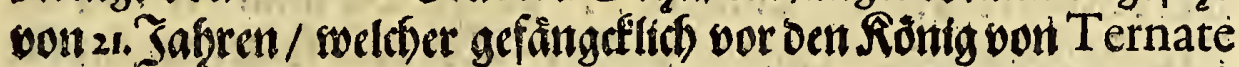

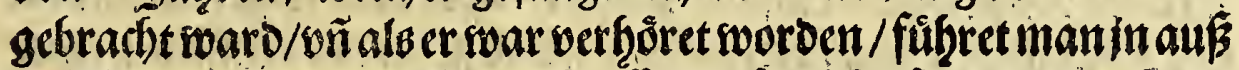

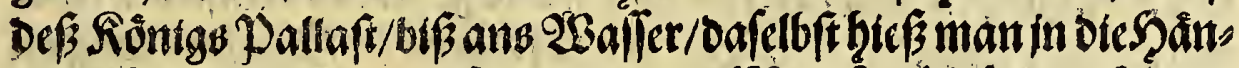

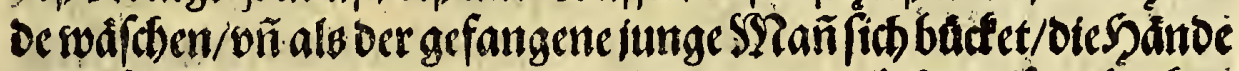

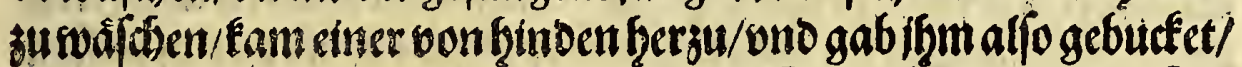

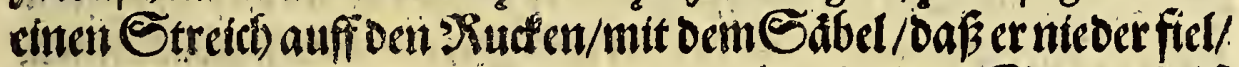

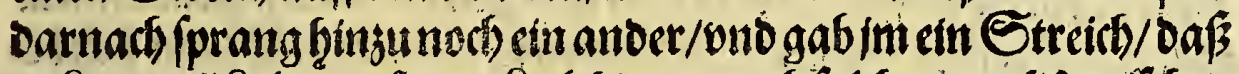

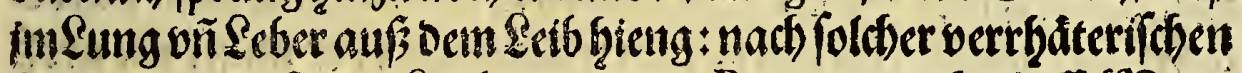

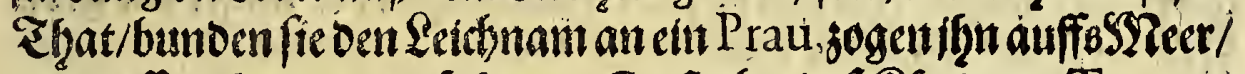

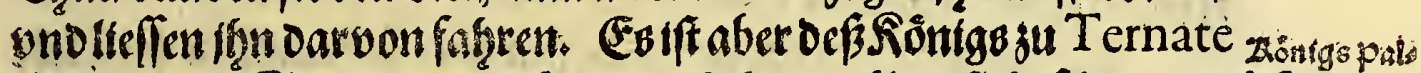

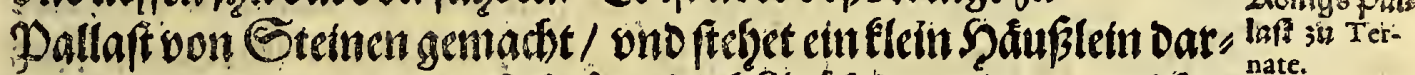

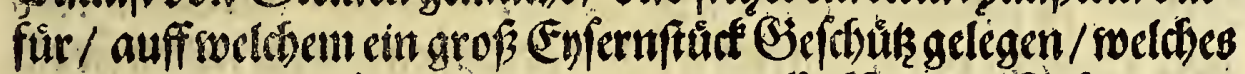

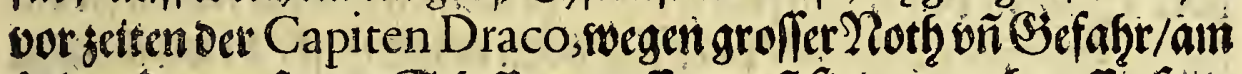

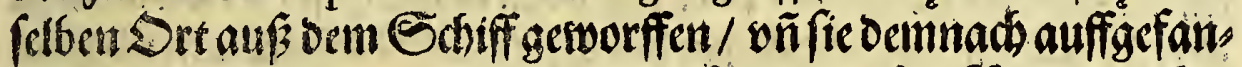

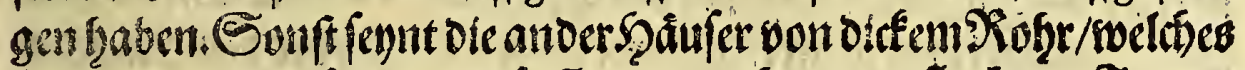

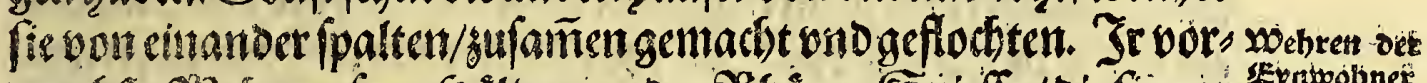

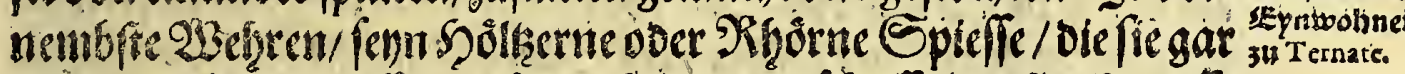

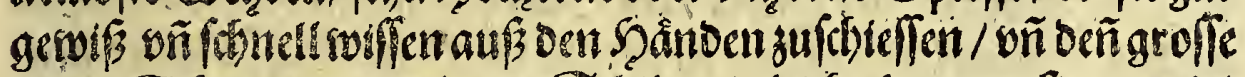

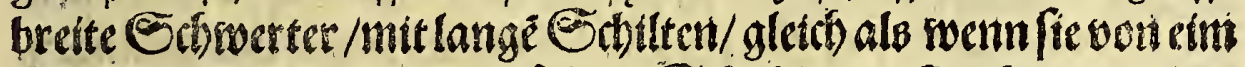

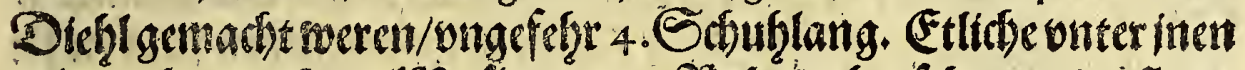

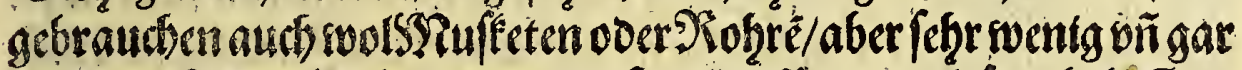

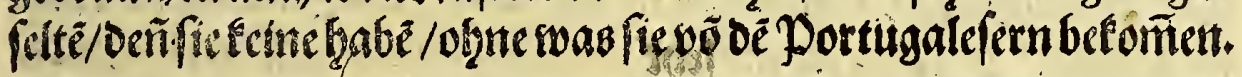

QBañ es firf begibt/oaf cinfinftermus an ber Soñê ober an

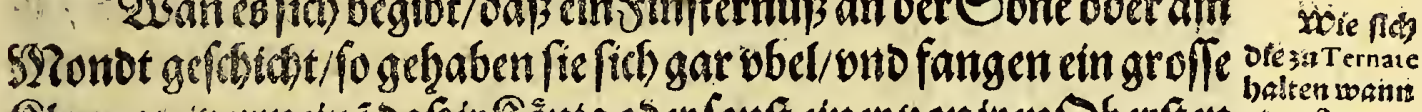

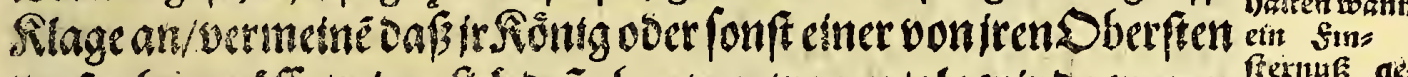

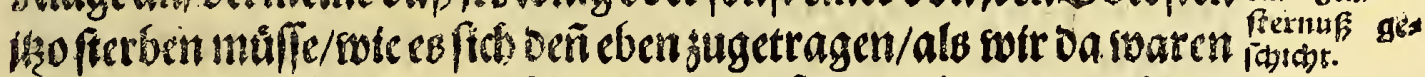

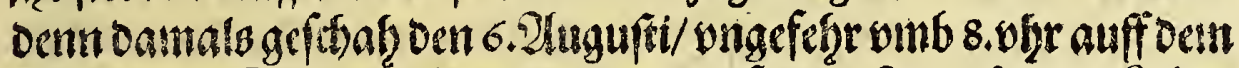

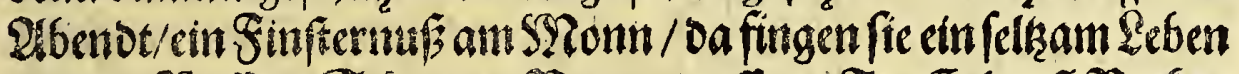

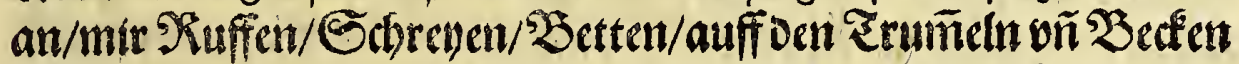

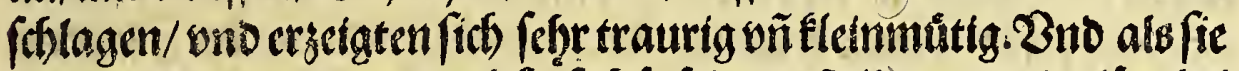
gefraget worben/ swarumb fiefies) fo felkam ftellèten / ond alfo obel

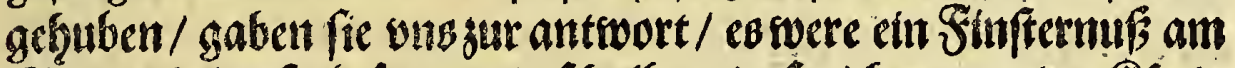

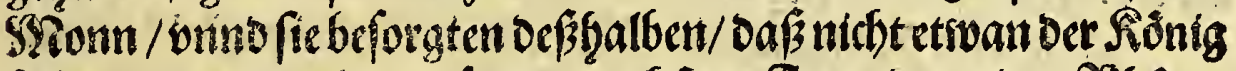
felbft / ober einer oon feinen ned)/fen freunden oder Sibäten

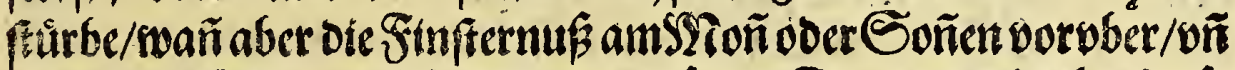
weder Der Ränignodf fonftiemande feiner Freundegeftorbe if / fo

Gi if ricften 
Srigboole ober Solbaten/onb nath ben Solbaten/ tompt etiter

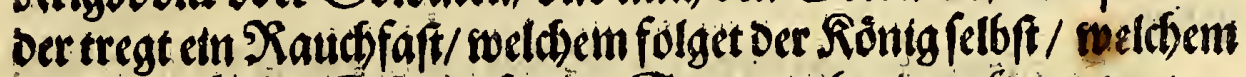
ein Tirefol boer Gibirm fưr bie Sonne / bber Dem Şaupt getras

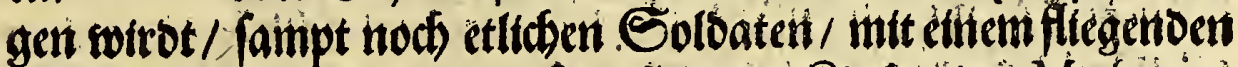

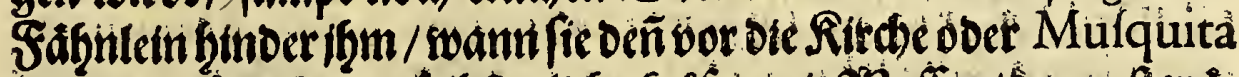

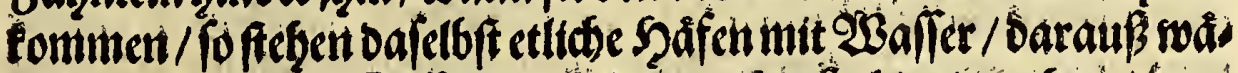

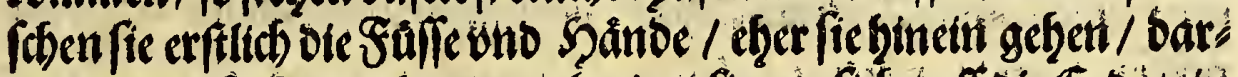

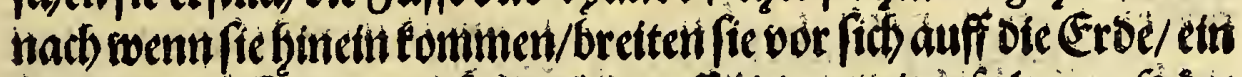

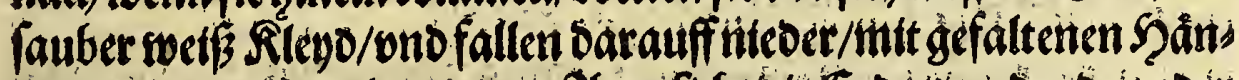

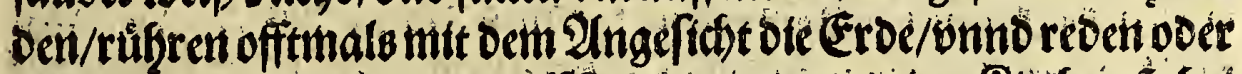

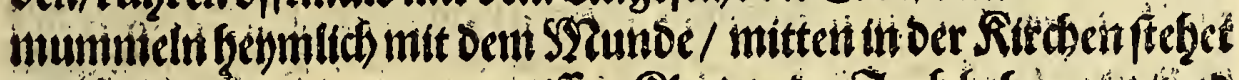

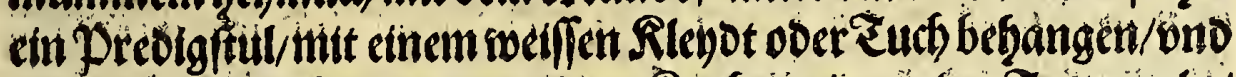

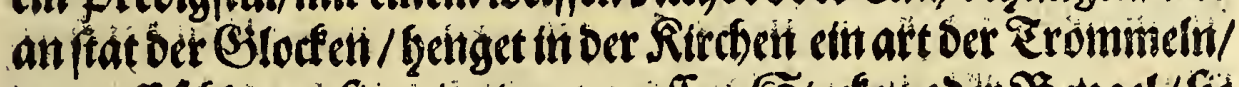

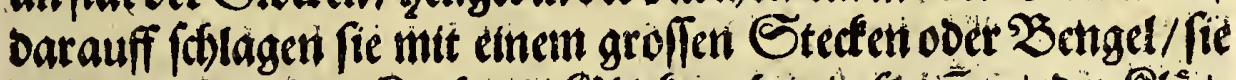

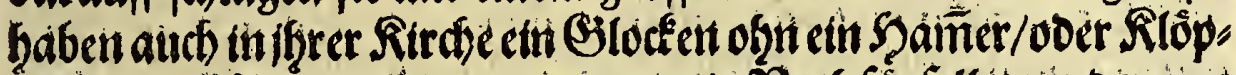

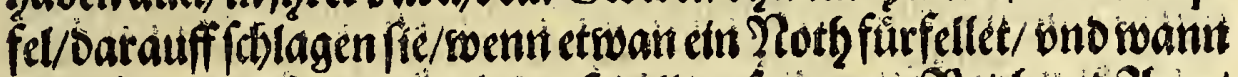

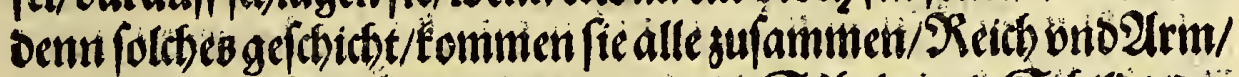

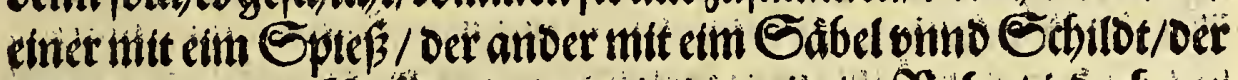

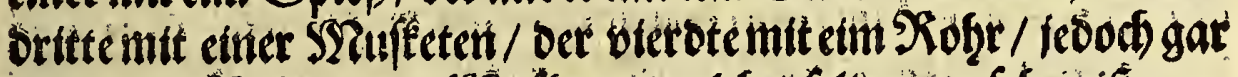

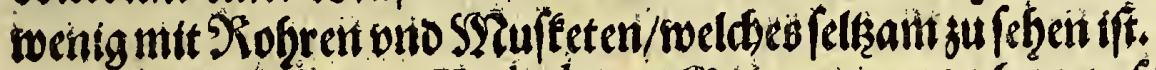

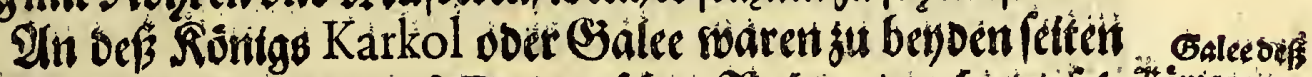

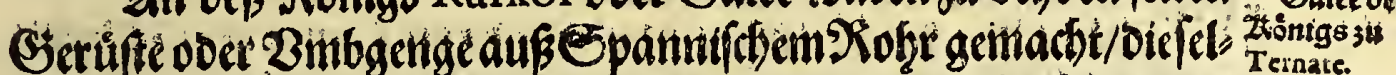

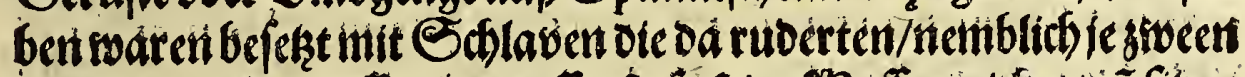

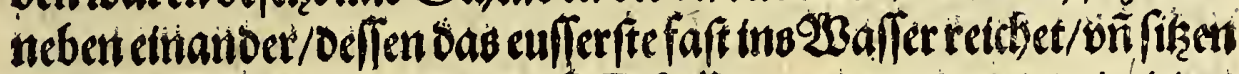

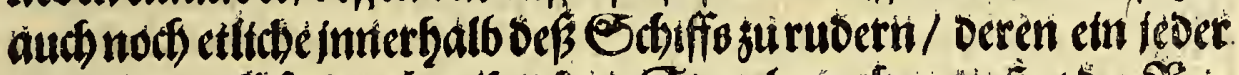

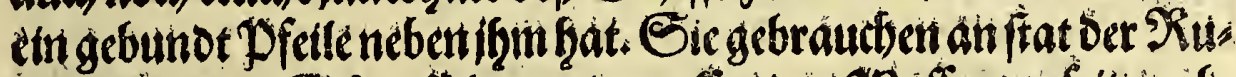

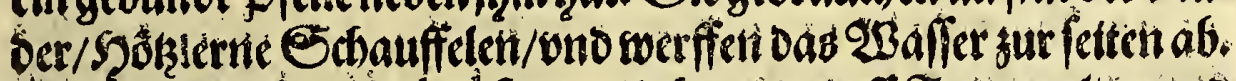

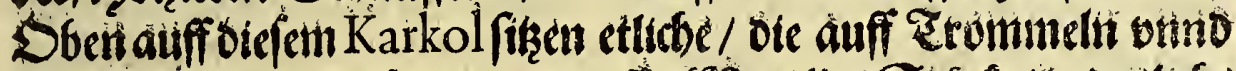

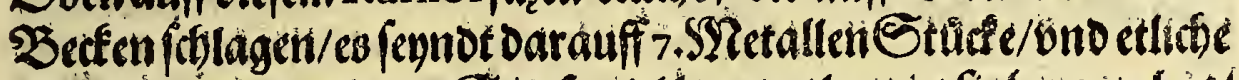

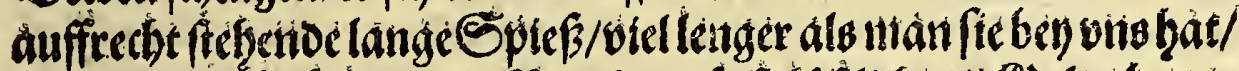

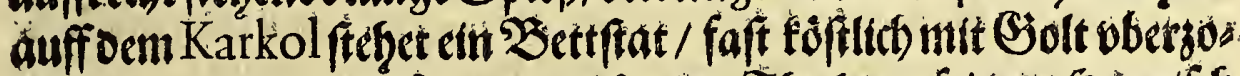

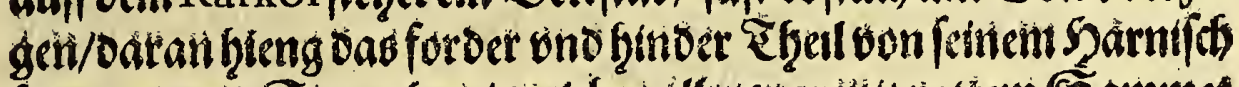
fampt einemi Sturmbut / matcheb alles war int rottem Sammet

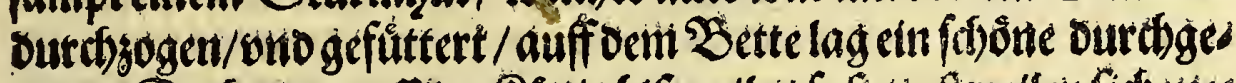

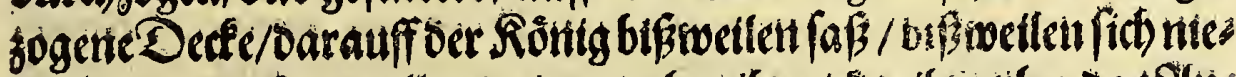

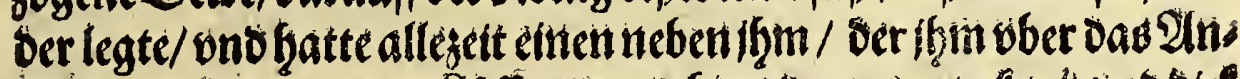

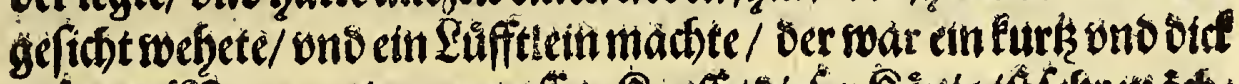

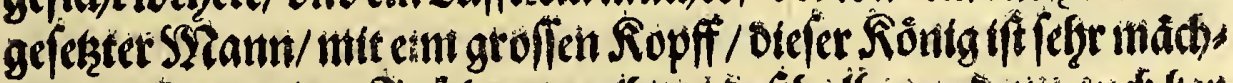

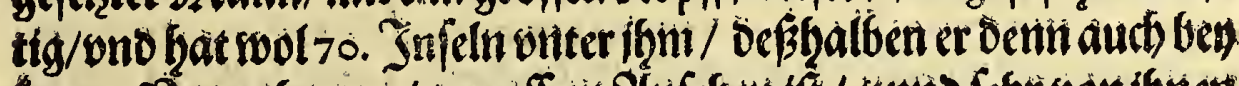

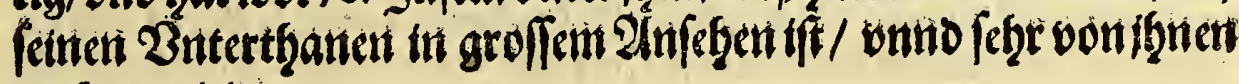
gechęet swird:

(3) iij Dom 


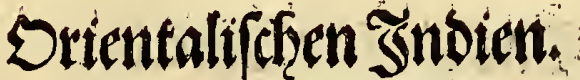

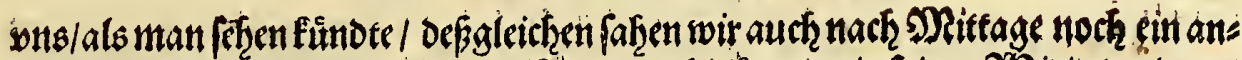
Dere Teuwersbrumft/it offenbarer Eec/wir aber hateen ein feinen Windt/ wh was

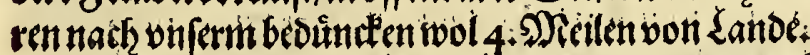

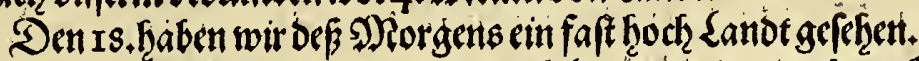

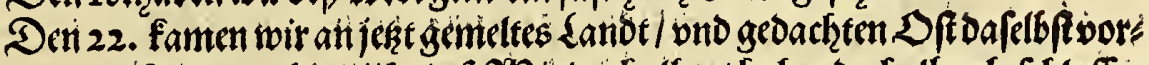

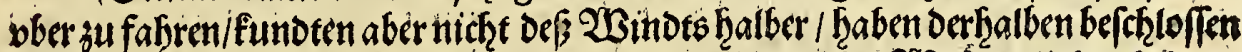

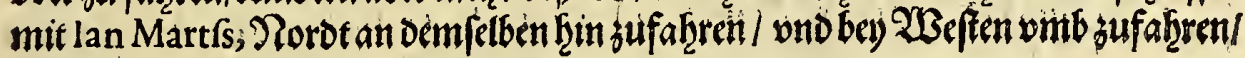

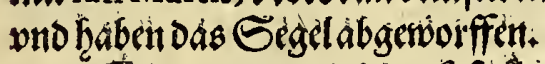

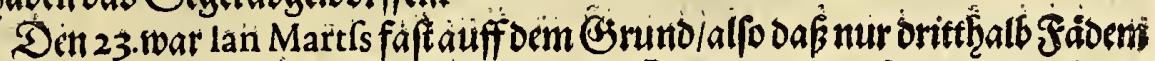

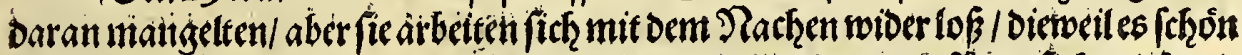

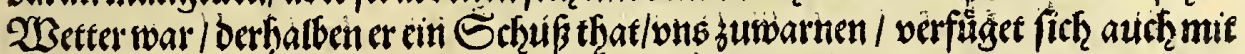

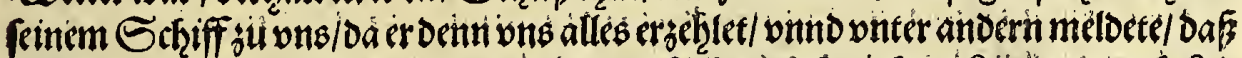
fie rool 6.Prauvven gefehedr hedten/aber mit fetinen gefprochen / funoten auch feis

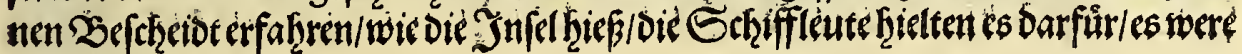
Die Irfel Boere.

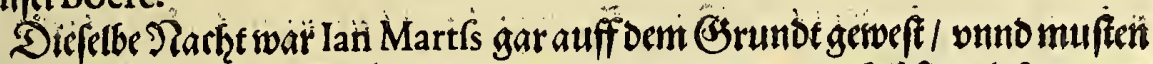

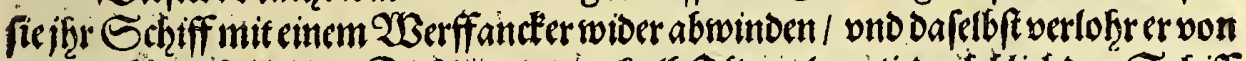

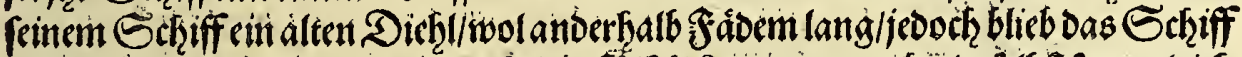

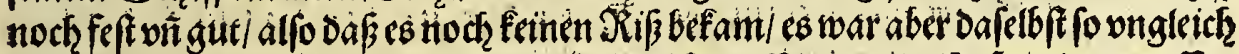

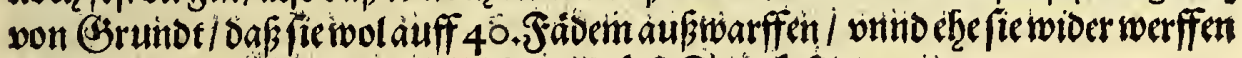

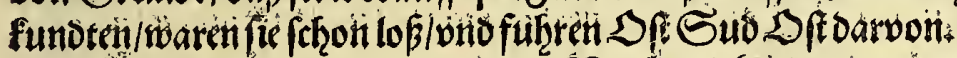

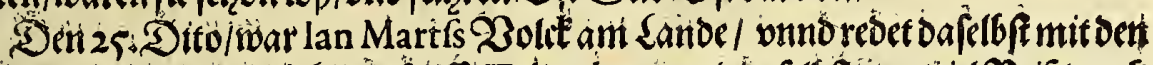

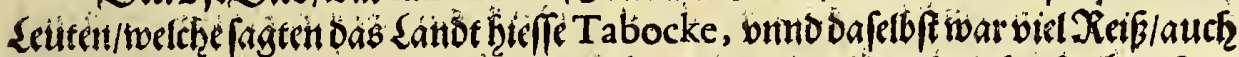

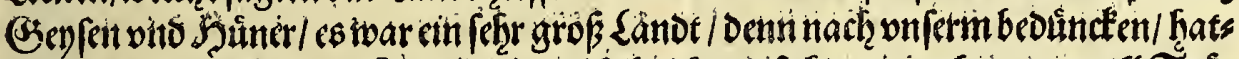

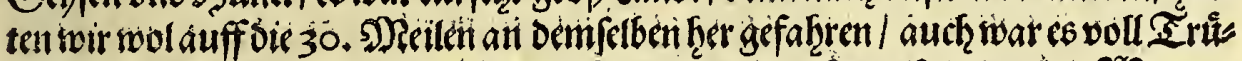

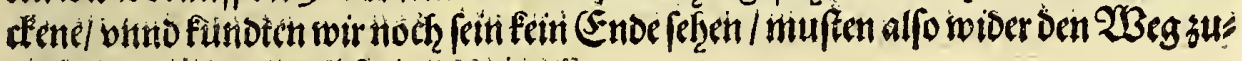

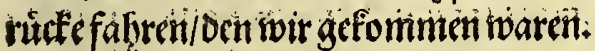

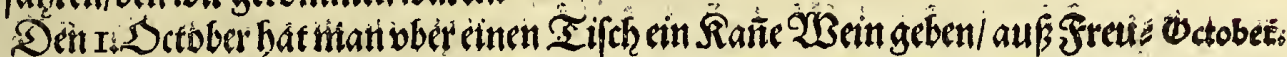

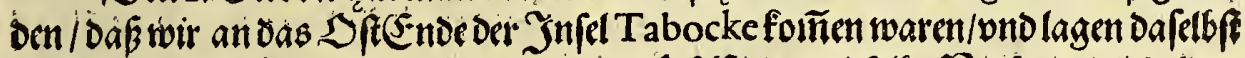

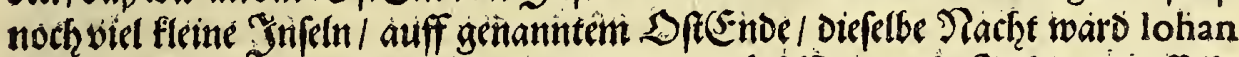

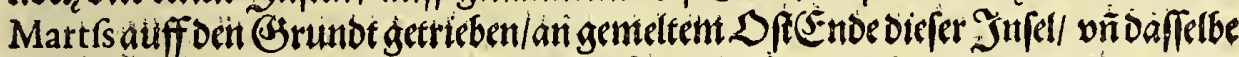

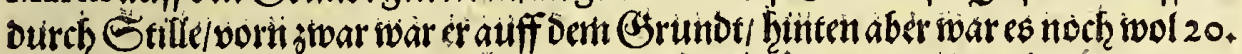

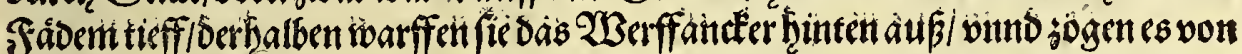

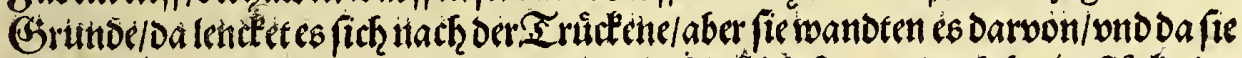

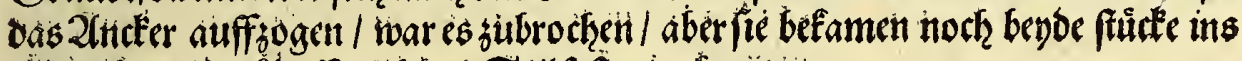

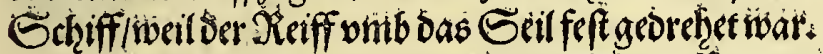

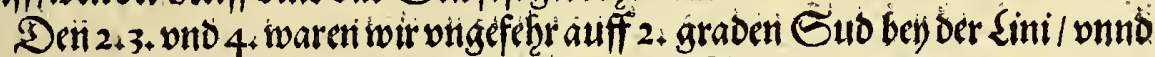

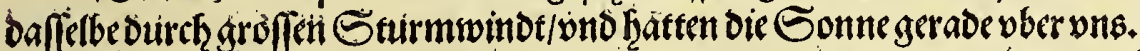

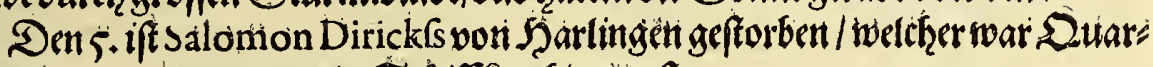

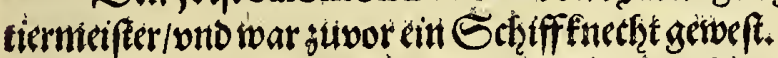

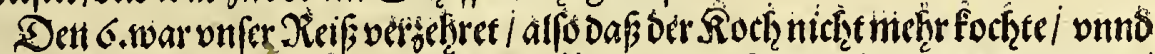

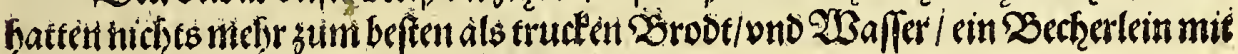

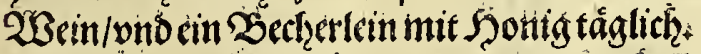

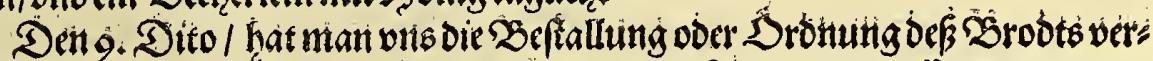

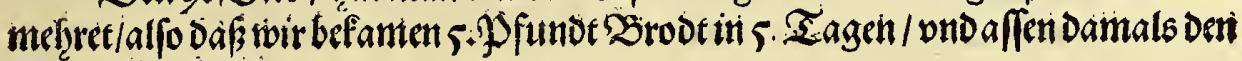
leşen Gtodtifict:

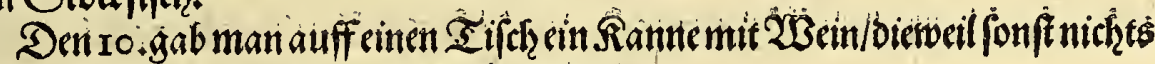

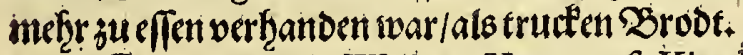

Den II. natot Willem Hermanfs Klock von Zlletmaer jum Duartier

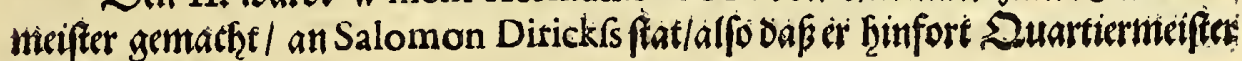
bno 23 enbel rajar.

Dิet 


\section{Erientaliff̧en Inbien.}

Das \{antot Madura, ba wirwaren gefangen geweft/ vno befunben als wir bas 3 len) auswarffer/bir Sieffevon 40, vno 50. Sádem.

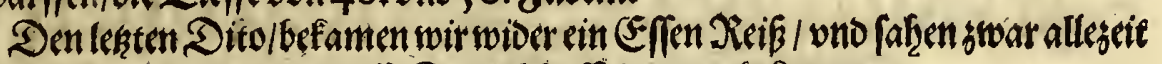

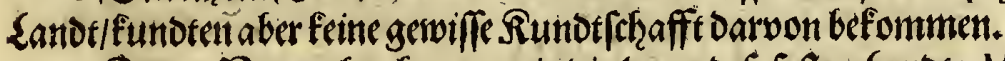

Den 2. Nouember hatten wir bie lenge deß feften Sandes Madura erreichtit/ thouembet.

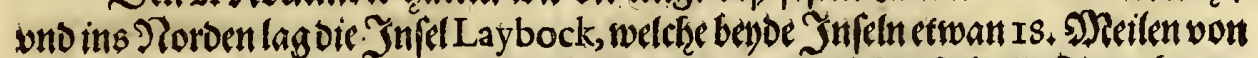

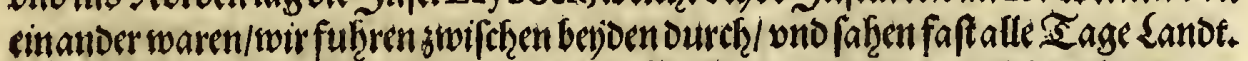

Den $5+$ Dito fuhken twir neben der $\mathrm{Yn}$ fel Carman Laue, welefre gelegen von

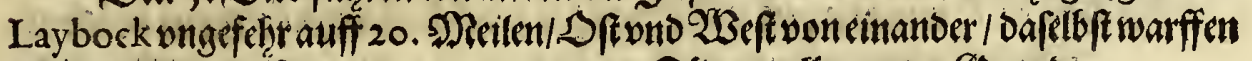
wir Das SEley auff $30 \cdot 35 \cdot 40 \cdot 45 \cdot$ vito 50. Sádem/ alles guten Otrundt.

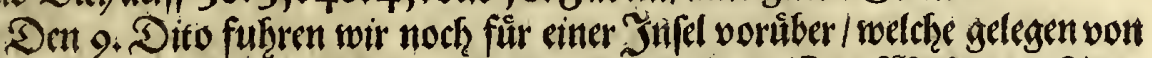

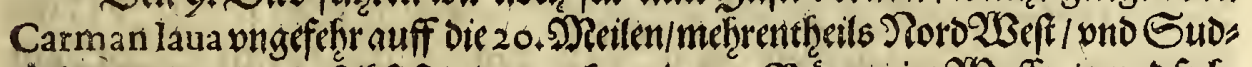

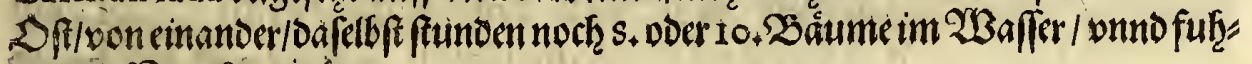
rentivir Rord ferumb.

Den I3. famen wir for Iaketra, vno befamennoch deffelben Tages de ir auff

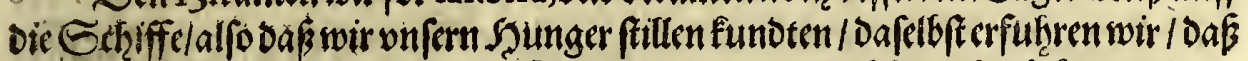

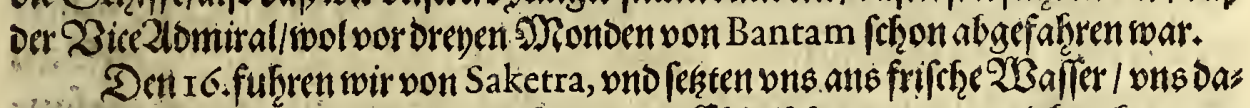
felbft mit 2 Baffer juverforgen / Da famen bie Chinifthen ju vns / vno brachten wns

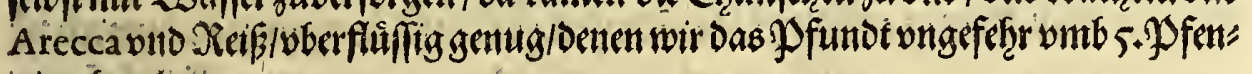
ting bejalteth.

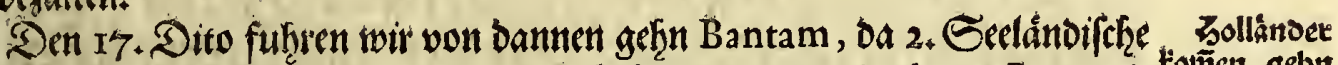

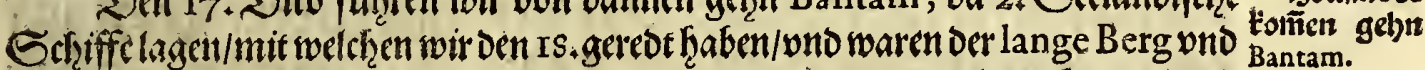
bie Sonne, die S. Mionoen vnnto jefien tage lang vor Bantam gelegen hiatten/ wno

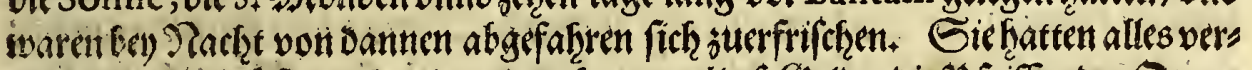

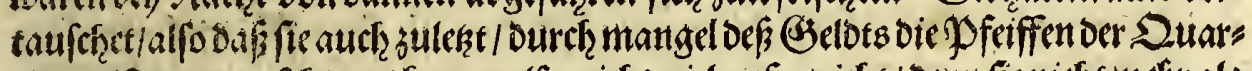

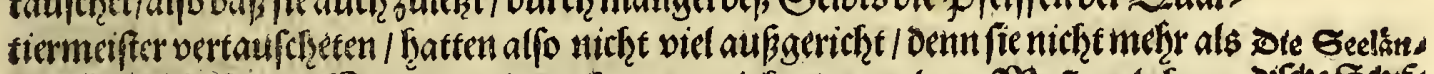

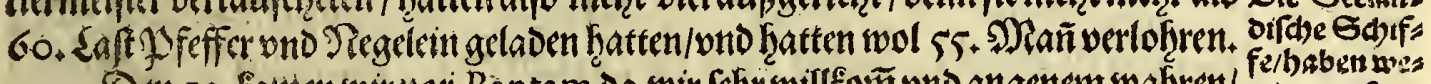

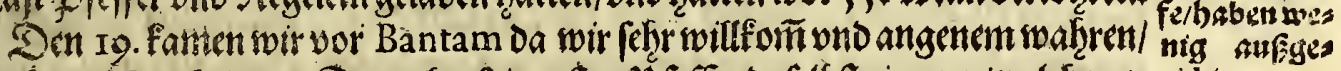

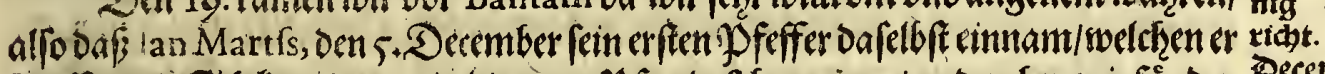

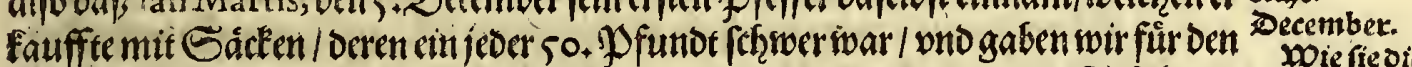

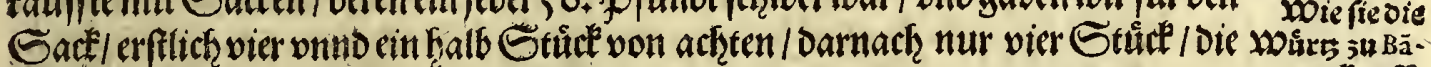

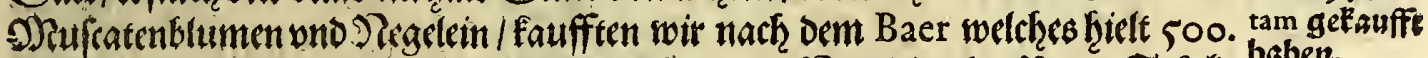

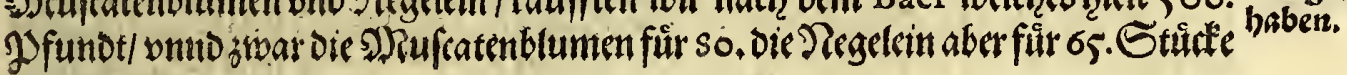
von achter.

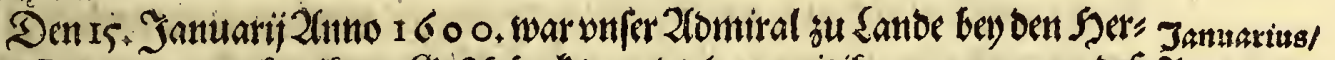

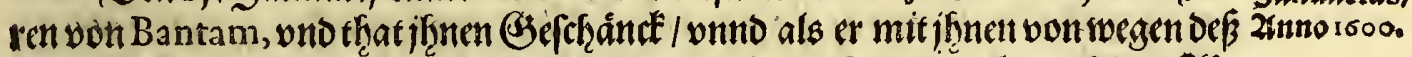

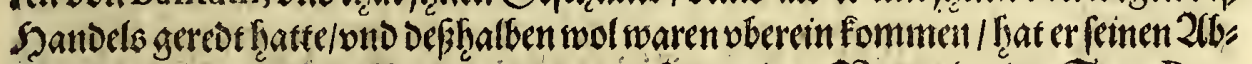

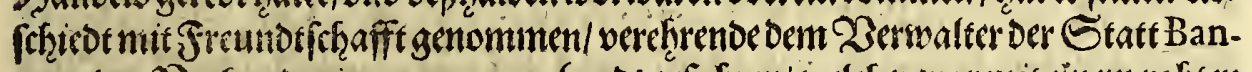

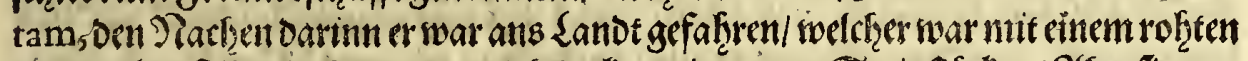

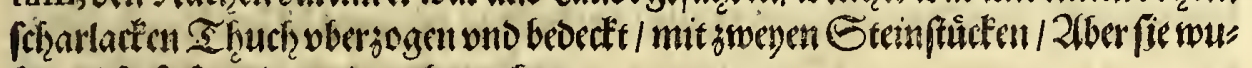
fien nicht faft wol oamit vimbjugetien.

Den 20. Dito frat man vnfer. Rauffleute wioer su Eefhiff getholet / ond ḩats en fic ibre 2Bahren faft alle verfauffit.

Den 21 . fuht noch ein Rauffmann ans Sande / mit einem wenig Sammet/

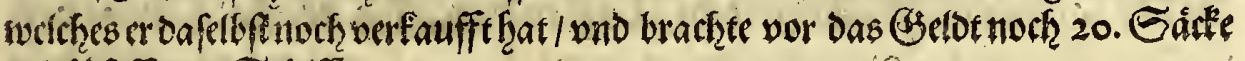
mit Pfeffer ju Echiffe.

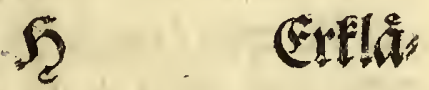




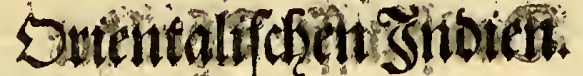

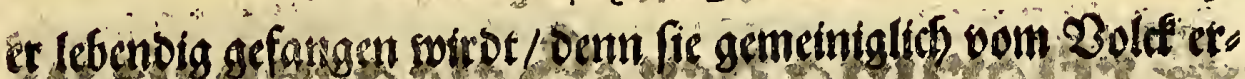

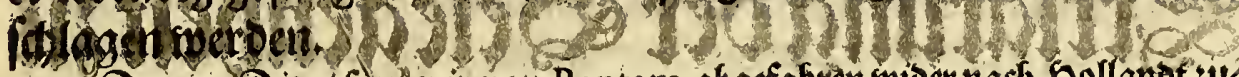

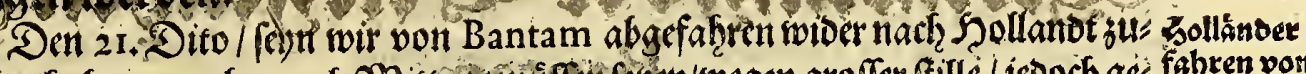

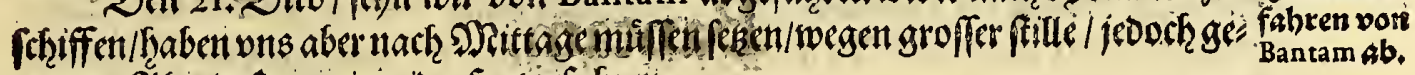
gen Den 2 bentot fend wir wider fortge faf̧ren.

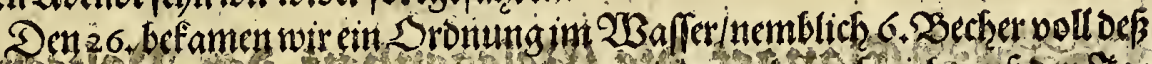

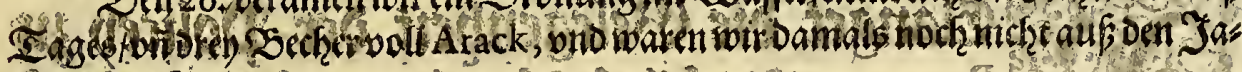

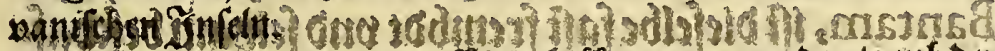

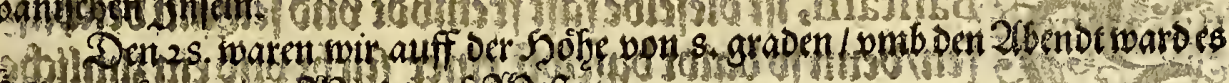
till

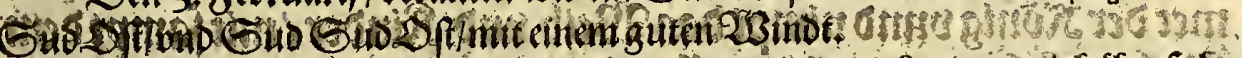

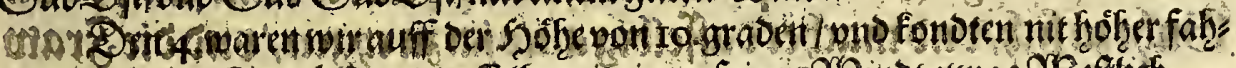

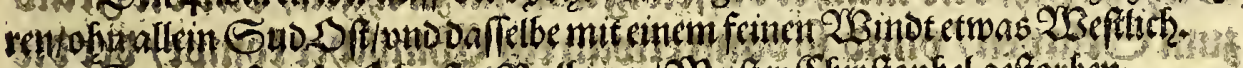

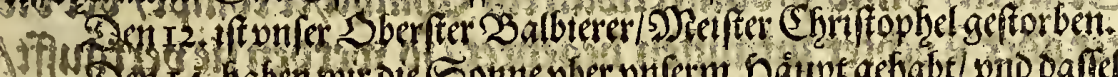

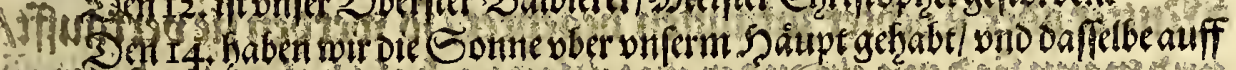

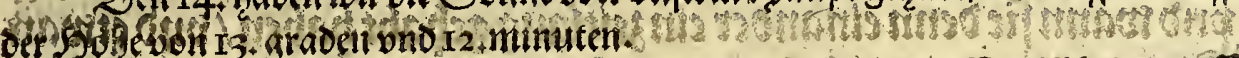

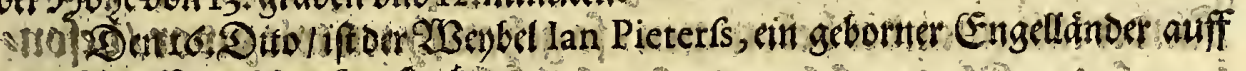
DenGofiffuari Marts geforben,

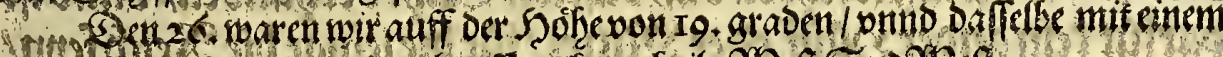

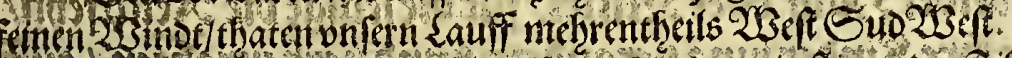

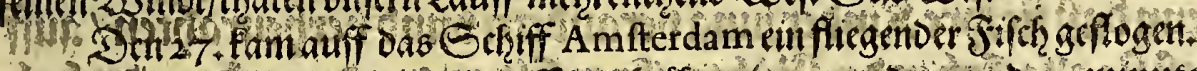

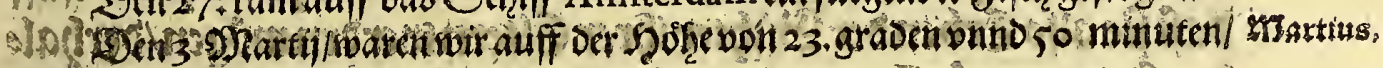

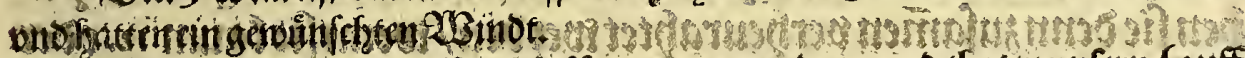

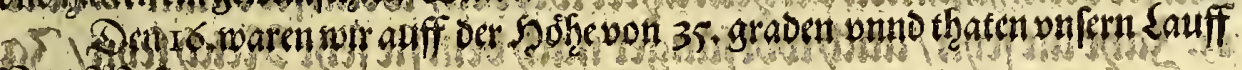

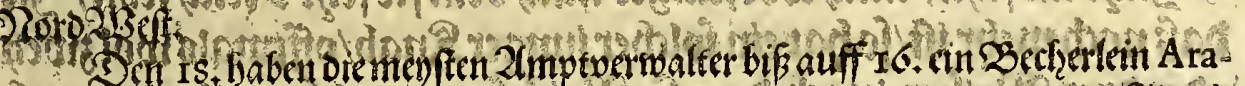
ckatermin

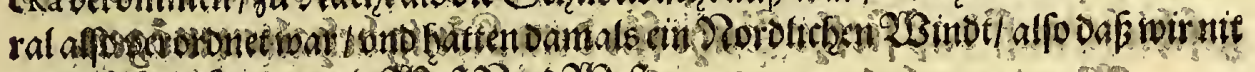

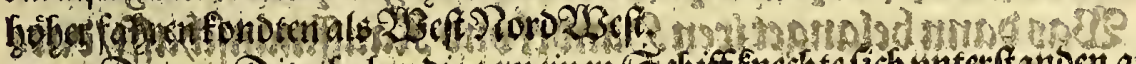

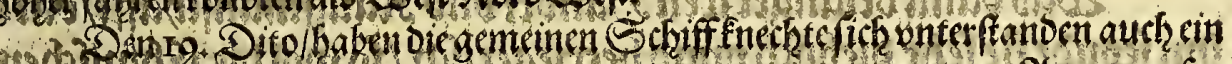

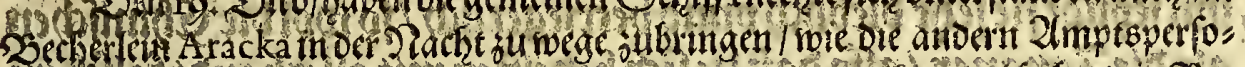

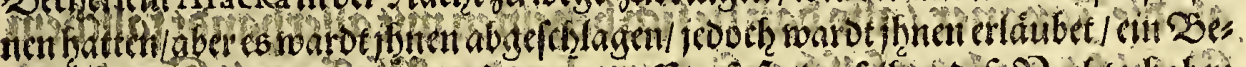

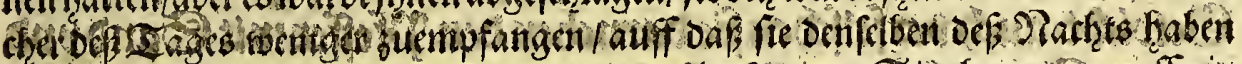

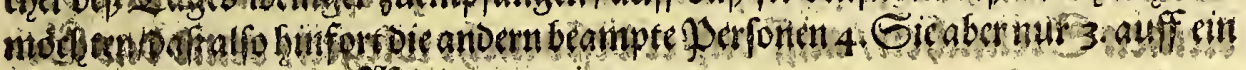

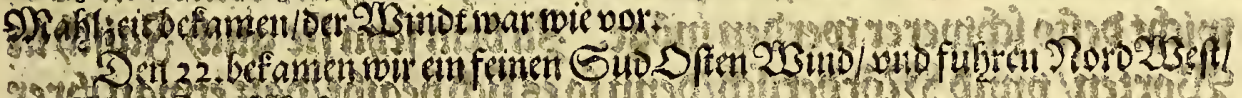

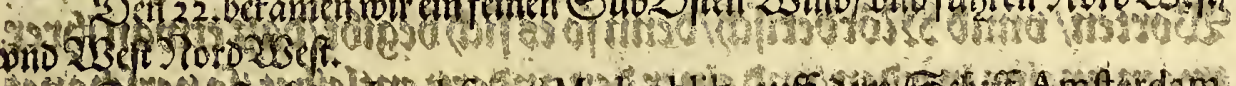
13

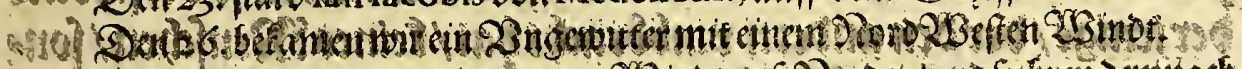

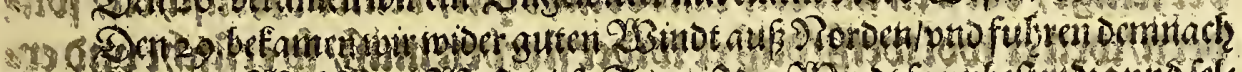

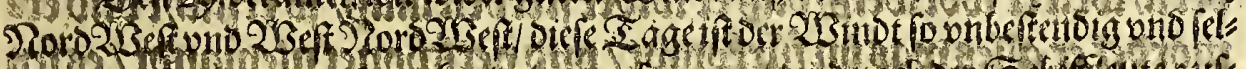

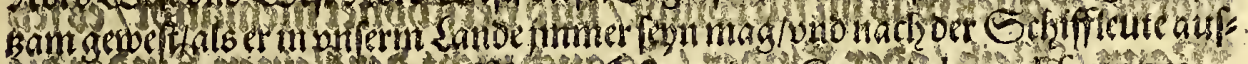

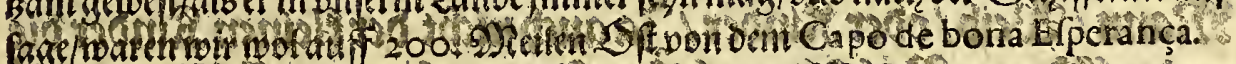

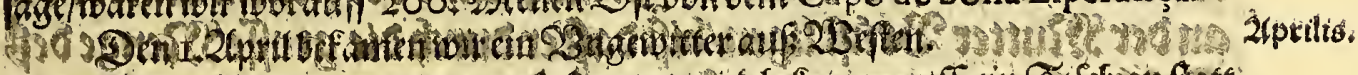

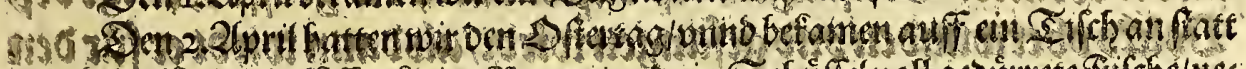

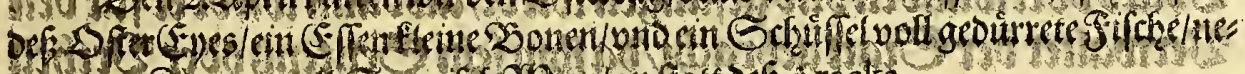

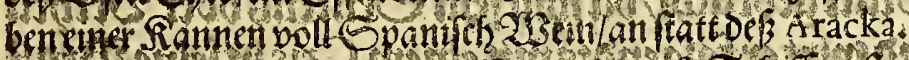

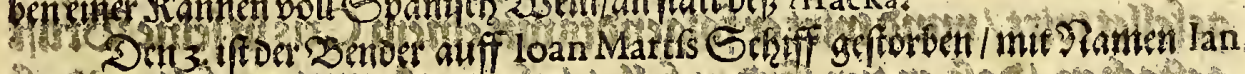

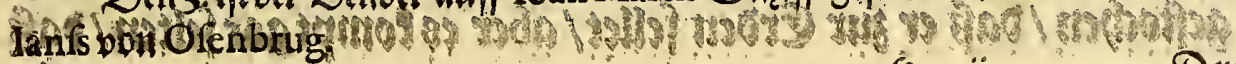
893

S if

Deit

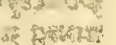
$x$ tertat

(1)




\section{Orientalifţen Sintien.}

WBas aber belanget die Infel S. Helena, ift daffelbeen faft Sruchtbar who des

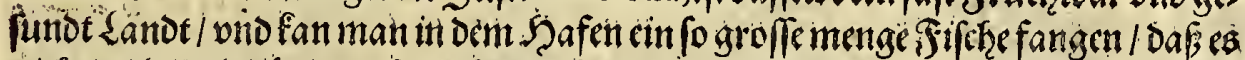

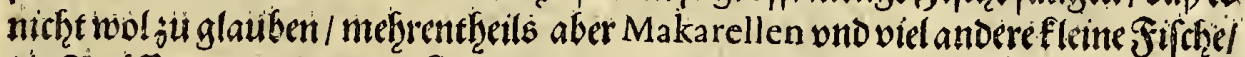

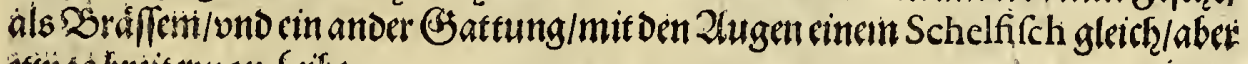
etriba brcitervon Seibe.

Den 25. Dito/waren wir auff ber Şofhe von 13. graden/ onno befamen beri 2Binde aus 1 Pord S ften/ wethret aber nit lange / foridern wendet fith bald wiber in

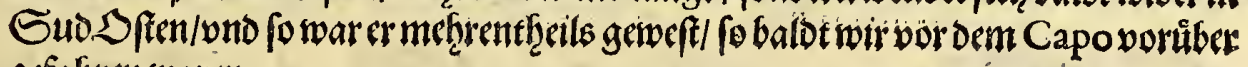
gefafiren waren.

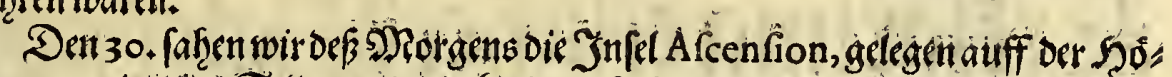

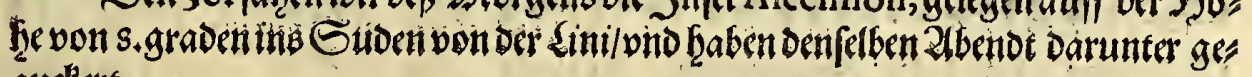
andlert.

VBir fersten autch in ber Nache etliche ant Sanot/Daffelbe subefichtigen / onno

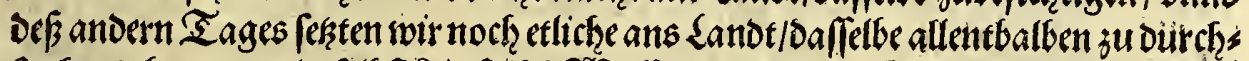

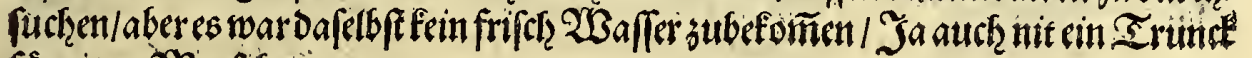
für einen Micnfthent.

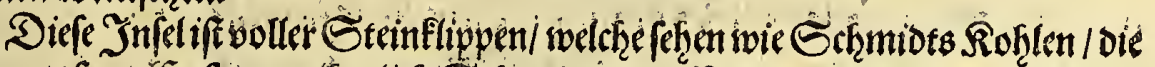

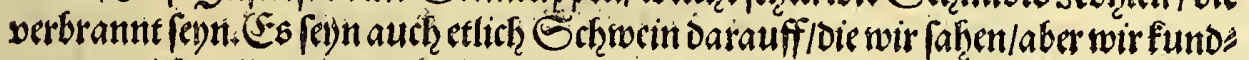
ten vils nicht griug verwundern ivorvon fie funden leben ond fith erhalten / fintes

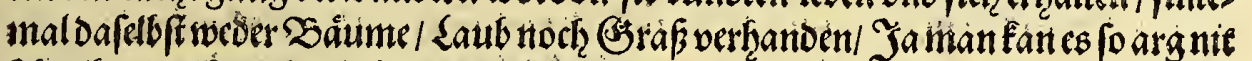

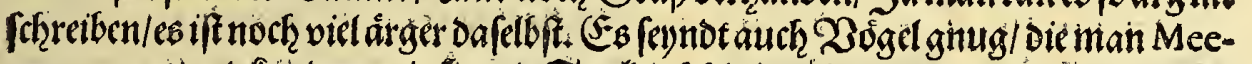

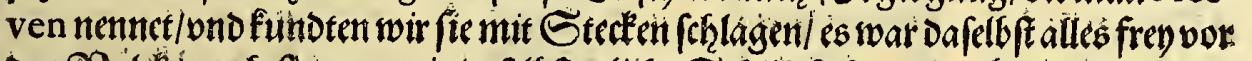
Das 2 old $j$ auch fiengen wir dafelbft etliche Echilbeffroften am Sanbe / Deren wit

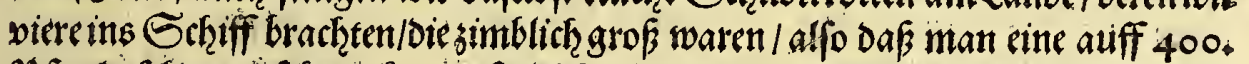

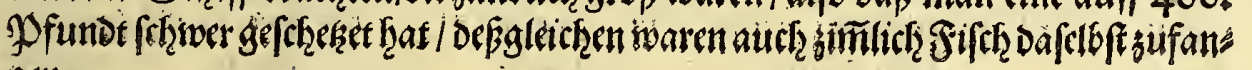
geil.

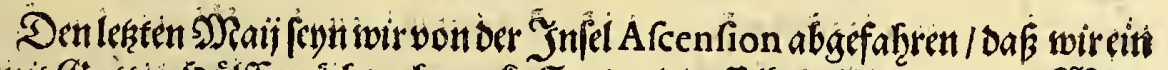

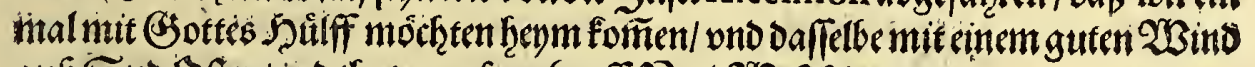
aus Guo Sften/ond thaten vnfern Sauff Pord Weft hineun.

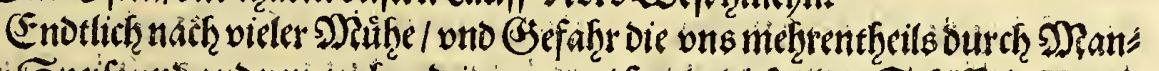

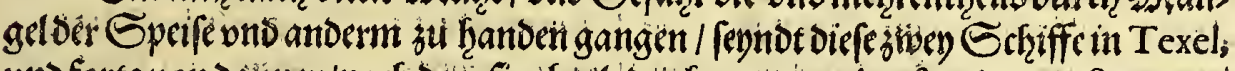

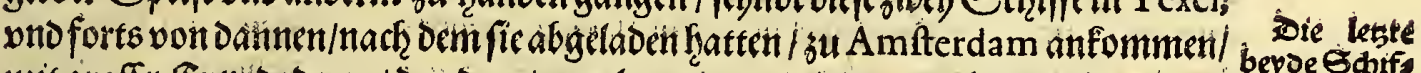

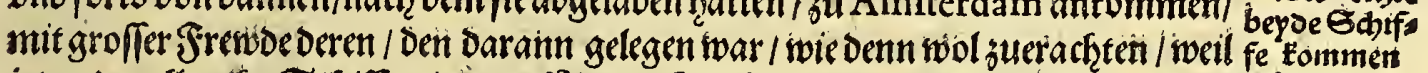

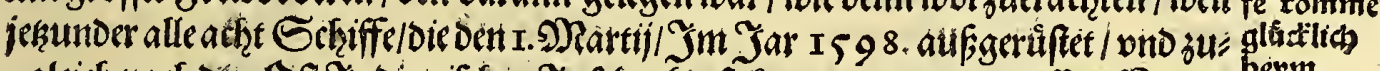

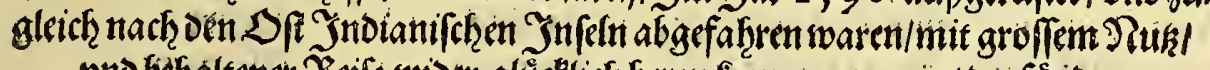

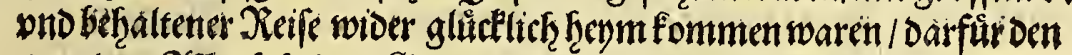

Dem2(llmáchtigen ङoft/Der fie fo wunderbarlich auff bem

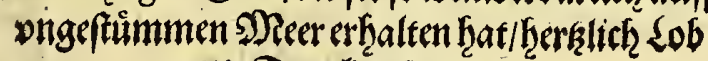
vind Dandt gefaget fey.

\section{$\mathfrak{H}$ iij $\quad \mathfrak{E r f f l a t}$}




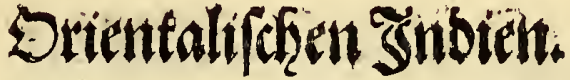

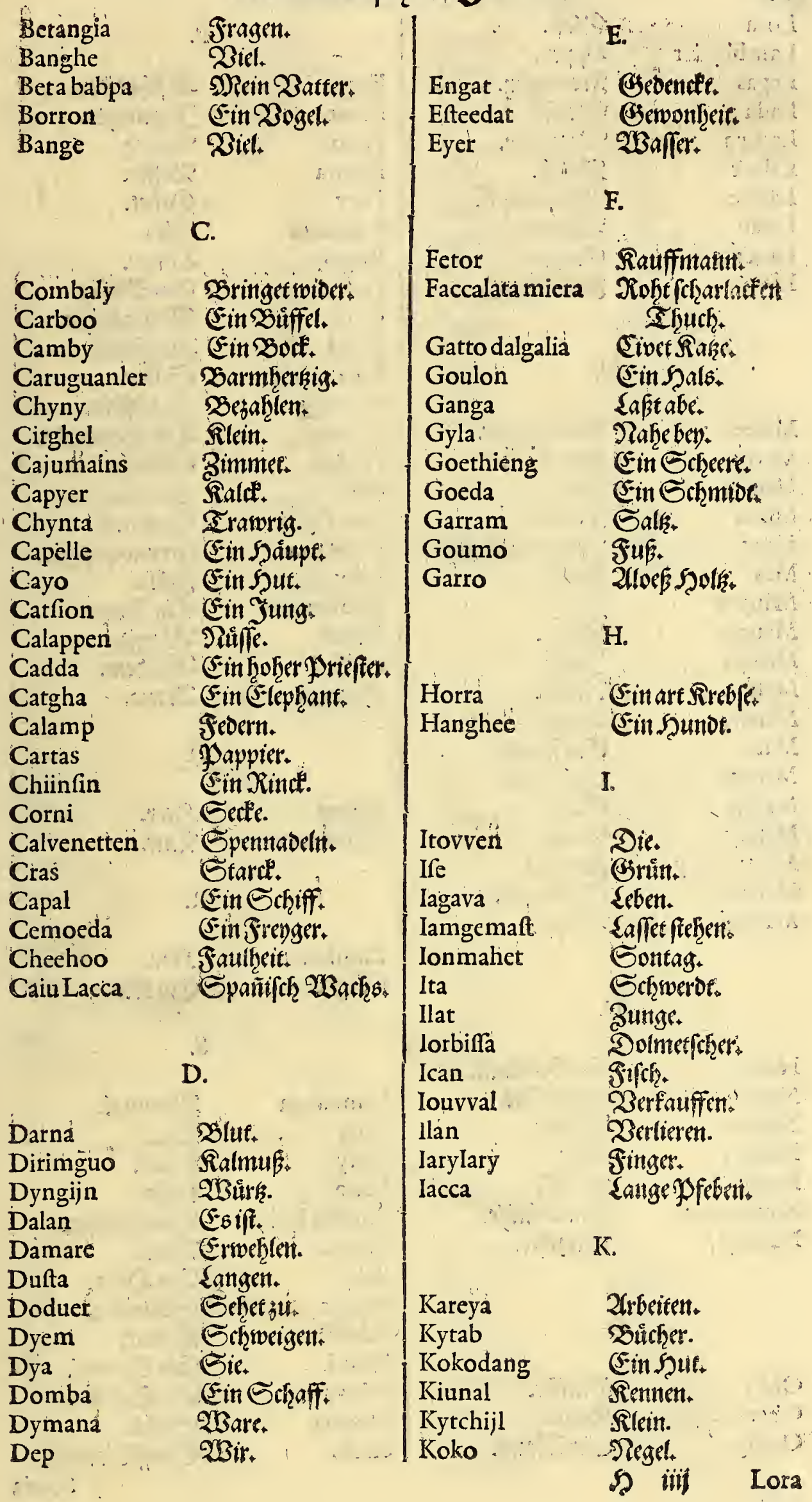




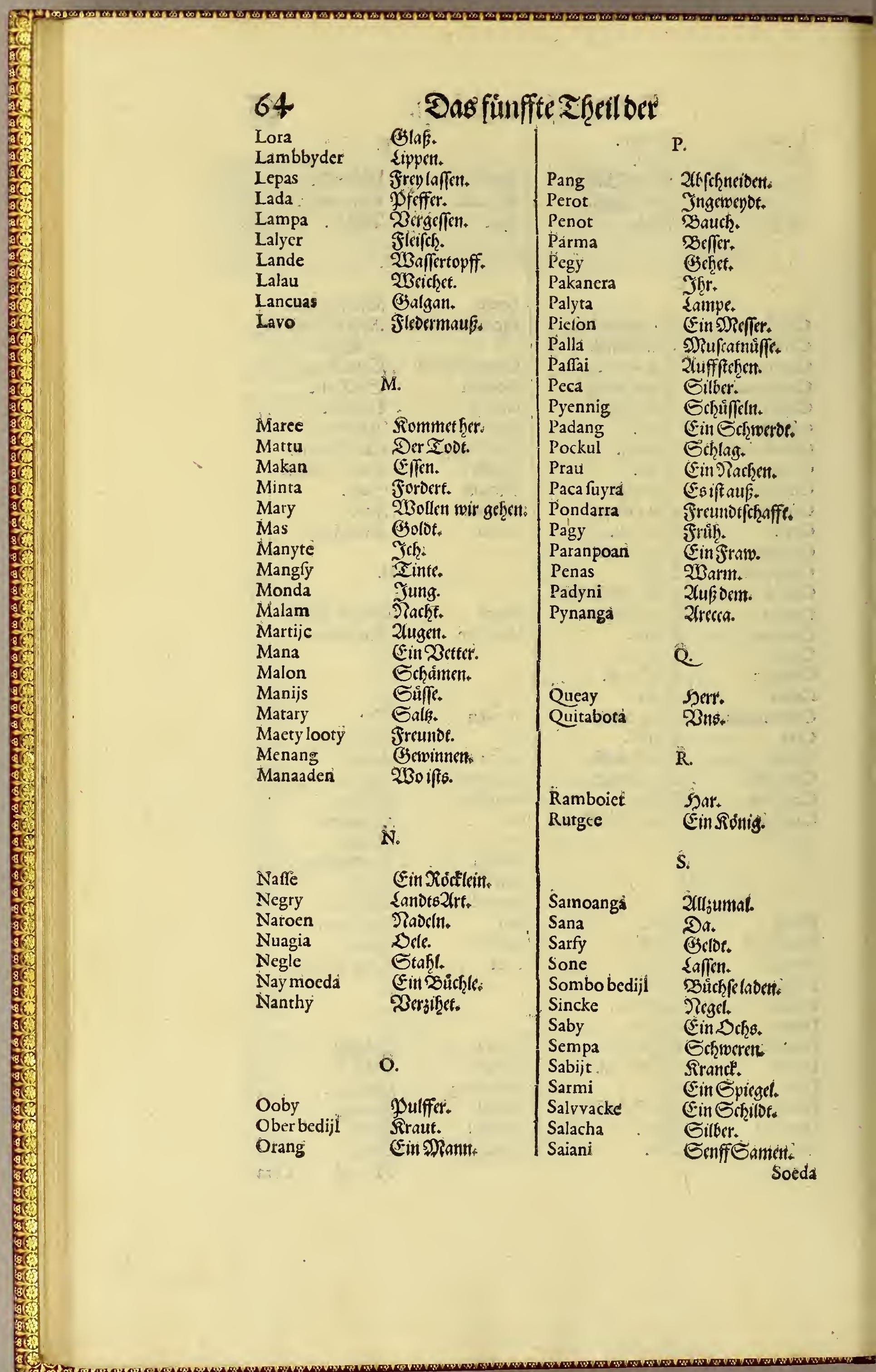




\section{Orientaliffich Jubten.}

\begin{tabular}{|c|c|c|c|}
\hline $\begin{array}{l}\text { Soedia } \\
\text { Saby } \\
\text { Selykit } \\
\text { Saya } \\
\text { Soufa }\end{array}$ & 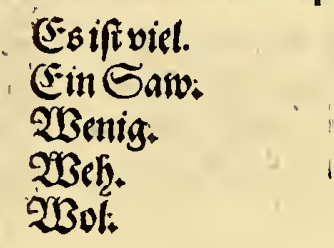 & $\begin{array}{l}\text { Tauvva } \\
\text { Tyeda tau } \\
\text { Tieda mau } \\
\text { Tiet ada } \\
\text { Tarrima Caffe, }\end{array}$ & 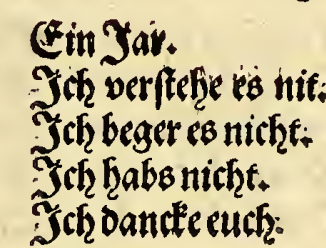 \\
\hline & $\mathrm{T}$ & $\begin{array}{l}\text { Tyma } \\
\text { Tieda } \\
\text { Taliinga }\end{array}$ & $\begin{array}{l}\text { Slen. } \\
\text { Neen. } \\
\text { Dfren. }\end{array}$ \\
\hline Tyngal & Defrut euch ๔̧ott. & Tua & $\mathfrak{Z l t}_{\text {t. }}$ \\
\hline Tganga & Ein Saart. & Tehylacca & Eolte. \\
\hline Tambagle & Ropffer. & Tomba & Sin Spief. \\
\hline $\begin{array}{l}\text { Terymacache } \\
\text { Tfuyka }\end{array}$ & Gott Dante auch. & Taly & Sin $5 \mathrm{e}) !$ \\
\hline $\begin{array}{l}\text { Truyka } \\
\text { Teloor }\end{array}$ & Sin Sebott. & Tácat & Sinfurcht: \\
\hline Tieda bayck & Tich̨t guht. & & 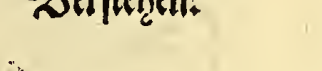 \\
\hline $\begin{array}{l}\text { Tabea } \\
\text { Tach ghỳ }\end{array}$ & $\begin{array}{l}\text { Ein guten Tag. } \\
\text { Gethet wes. }\end{array}$ & & $Y$. \\
\hline Toulong & Selffen. & Yfouck & Morget. \\
\hline $\begin{array}{l}\text { Tanga } \\
\text { Tage }\end{array}$ & Sin Shandor. & Yrot don & Paffe. \\
\hline 1age & & & \\
\hline
\end{tabular}

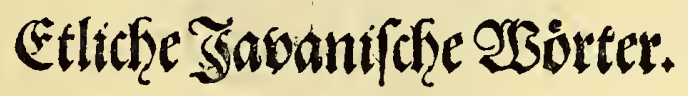

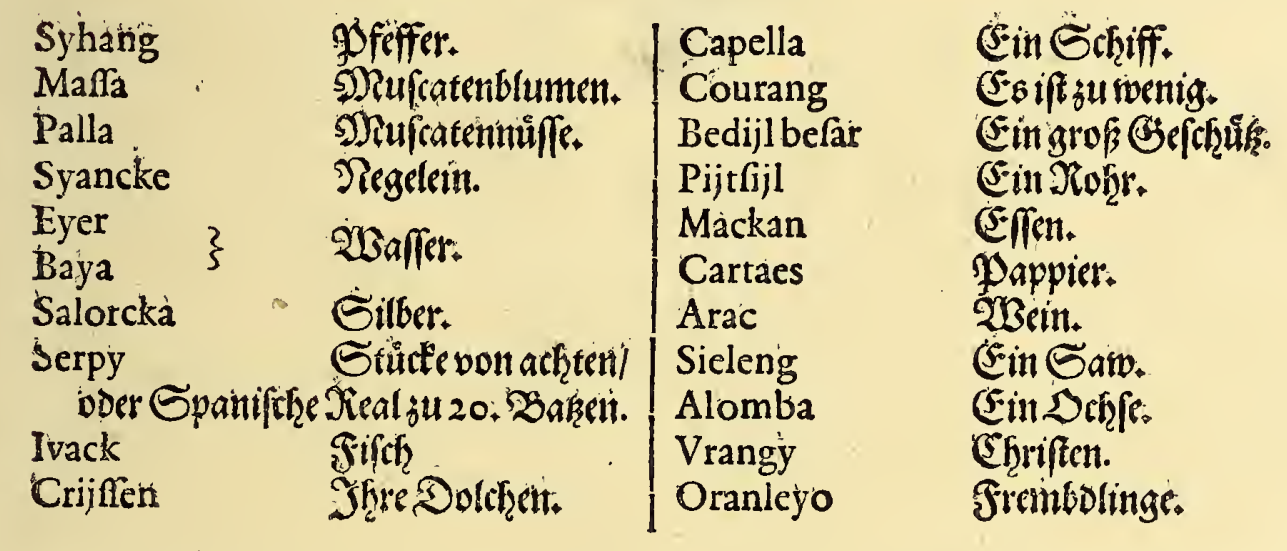

\section{Srolukifąe 3af̧len.}

\begin{tabular}{|c|c|c|c|}
\hline Satu & Eins: & Sambalan & Teune. \\
\hline Dua & Sorben. & Sapolo & 3efrn. \\
\hline Tyga & (S) rey. & Sabalas & Enlfie. \\
\hline En pat & Ditre. & Duabalas & Bwolffe. \\
\hline Lyma & Sunffe. & Tygabalas & Drevgehen. \\
\hline Nam & Erchles. & En pat balas & Dierkeferen. \\
\hline Toufion & Gieba. & Limabalas & Sunfferthen. \\
\hline Delappan & 2lchtct. & Nam balas & 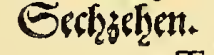 \\
\hline
\end{tabular}

Toufiou 


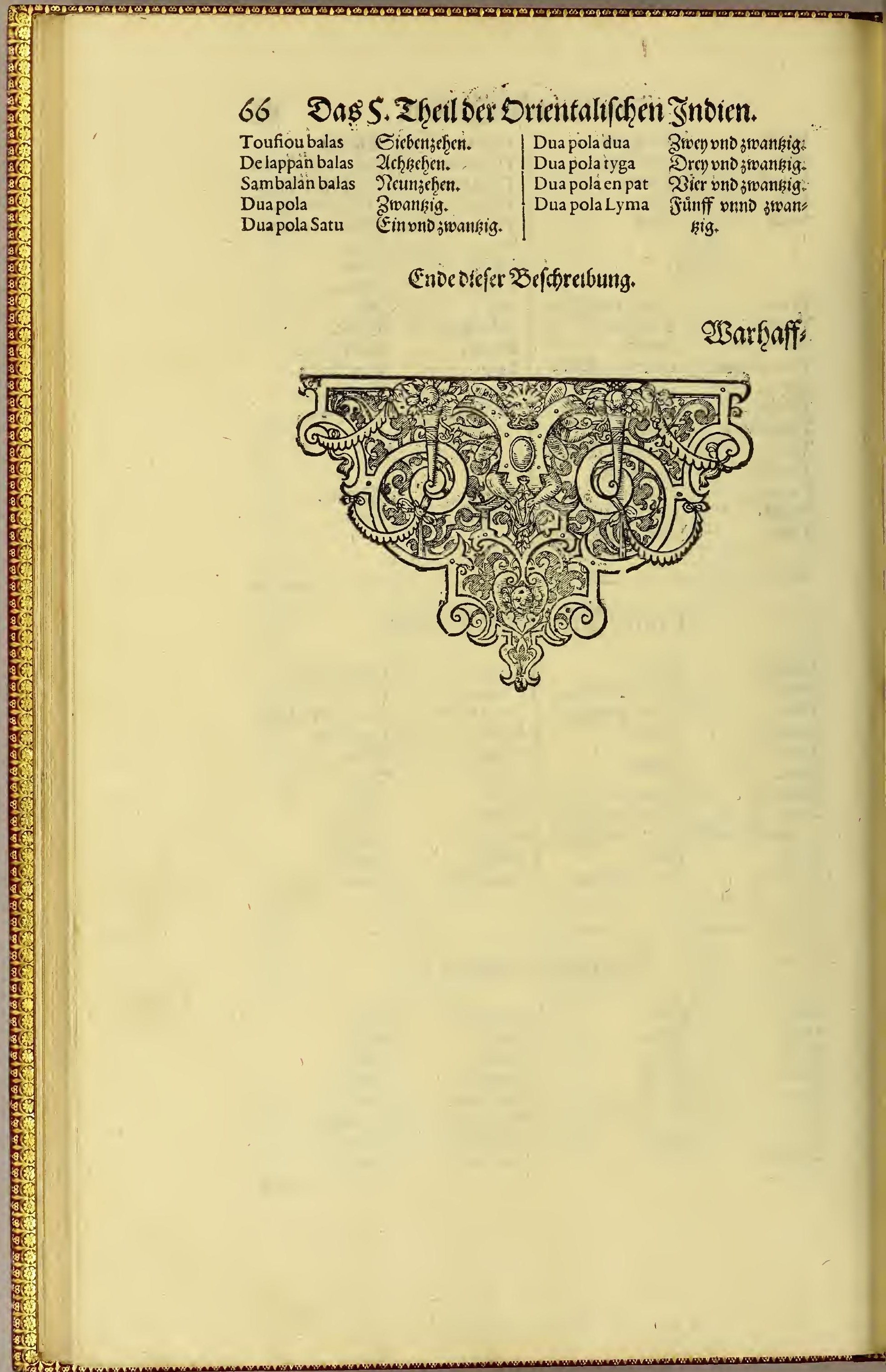





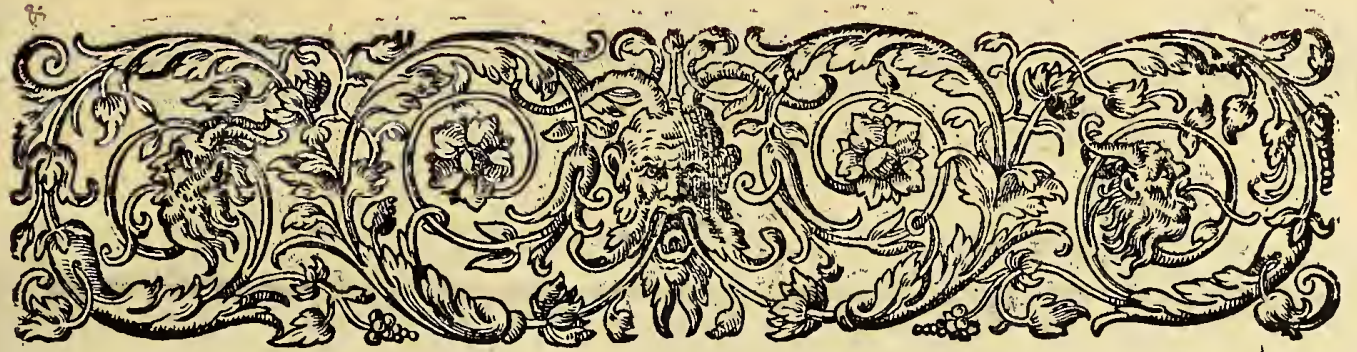

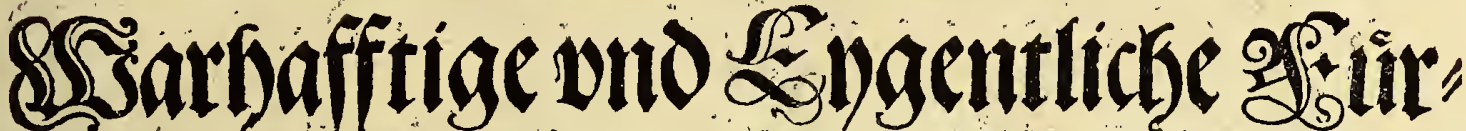

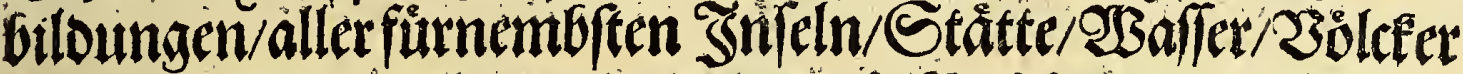

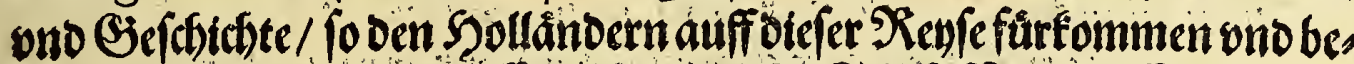

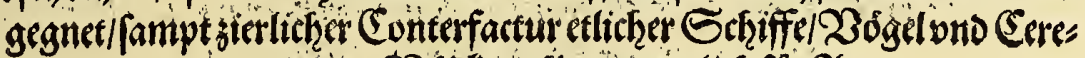

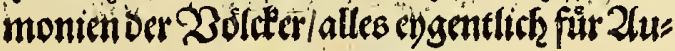
gengefellet.

\section{Sampt Summatifhem Snhalt onno Beffretbung ber Syt}

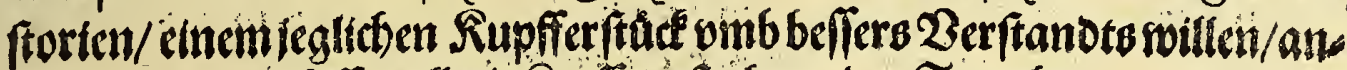

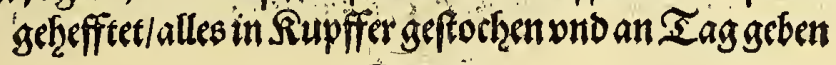

\section{Durcti}

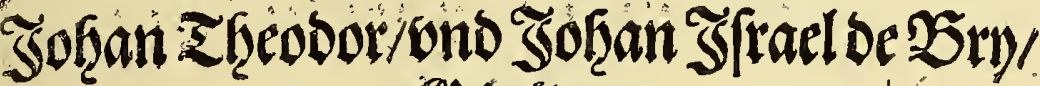
Gicbrubder.

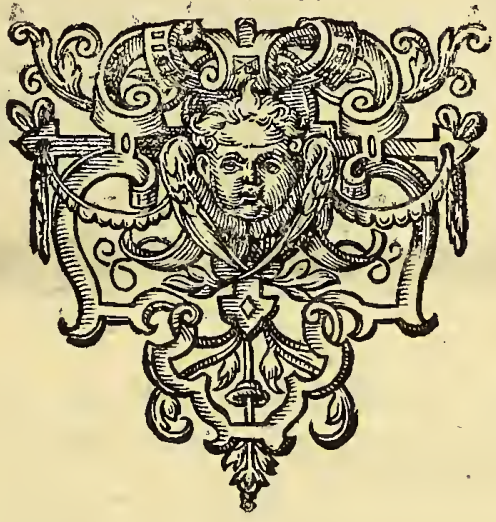

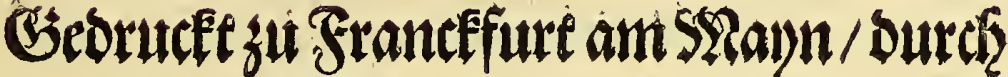
SRattheum $\mathfrak{E}$ effer.

$A \cap N O$ M. D C I. 


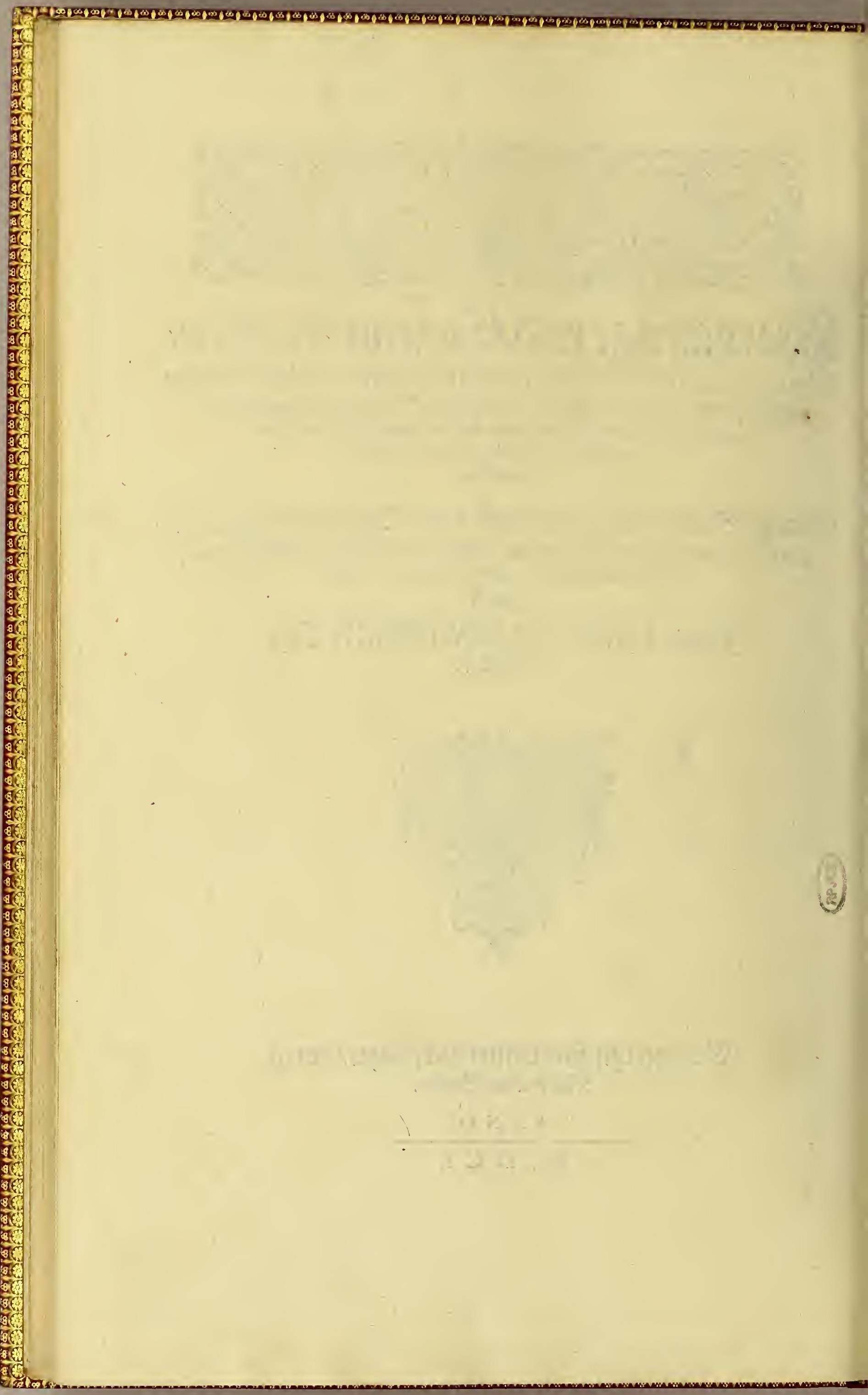




\section{Conterfactur der İ Mauritius genanne.}
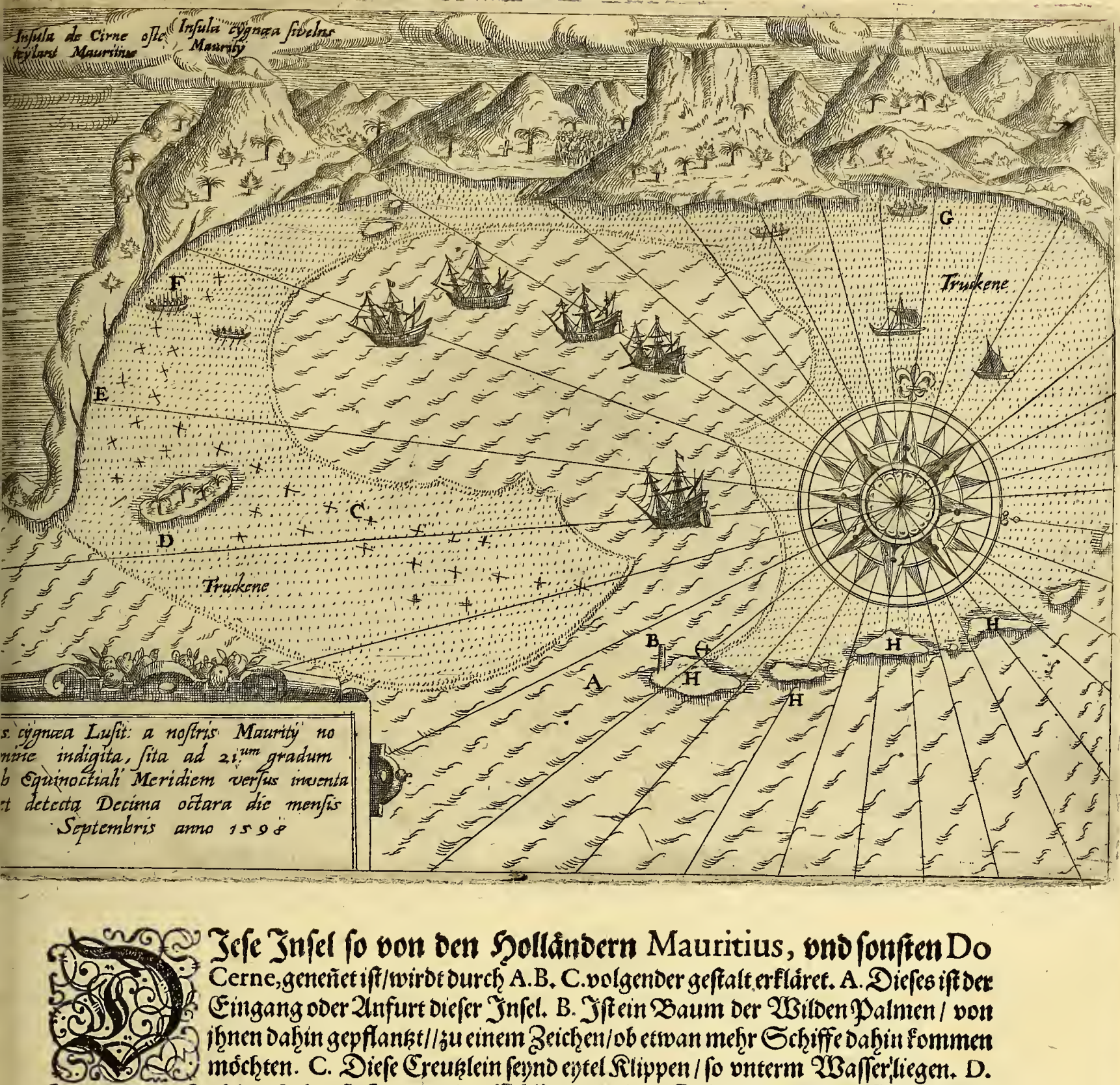

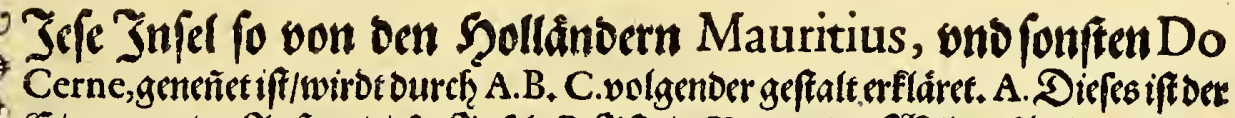

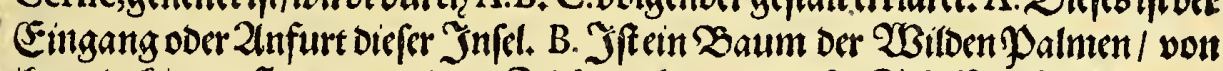

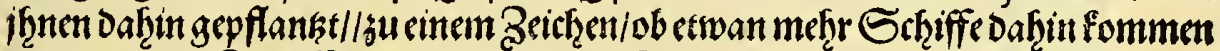

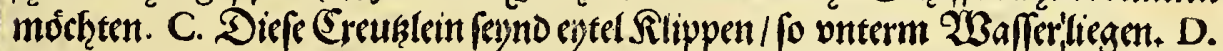

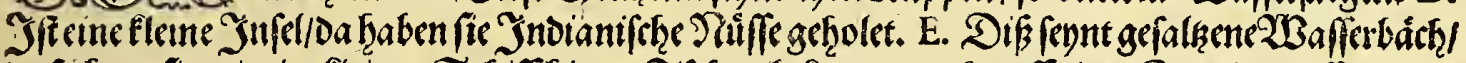

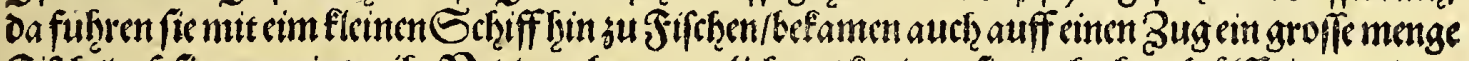

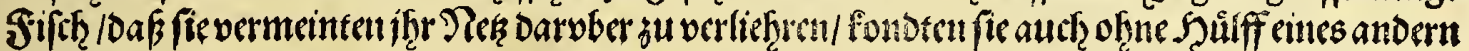

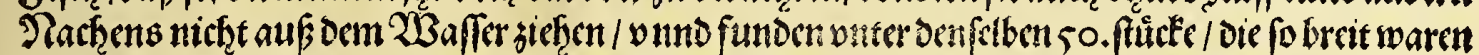

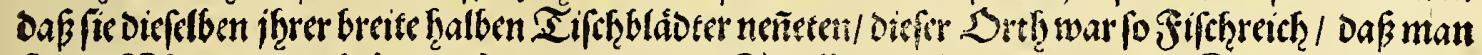

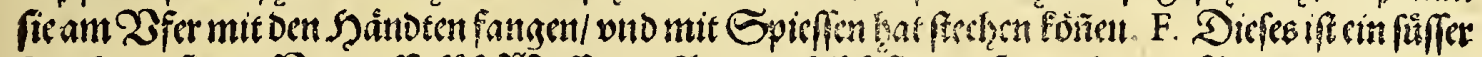

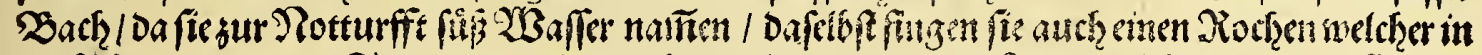

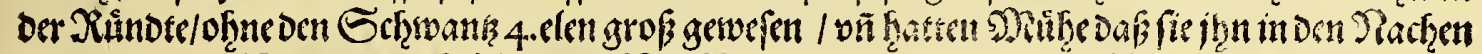

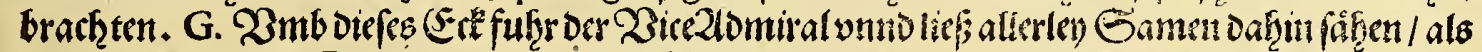

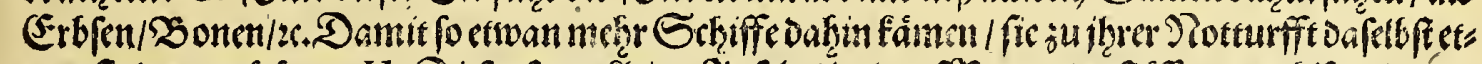

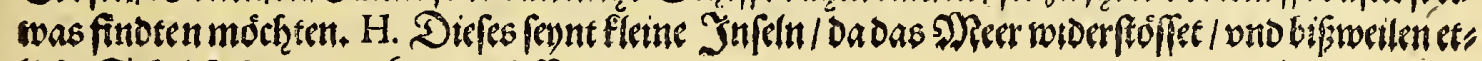

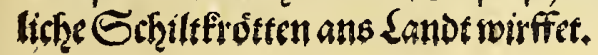

a ij 


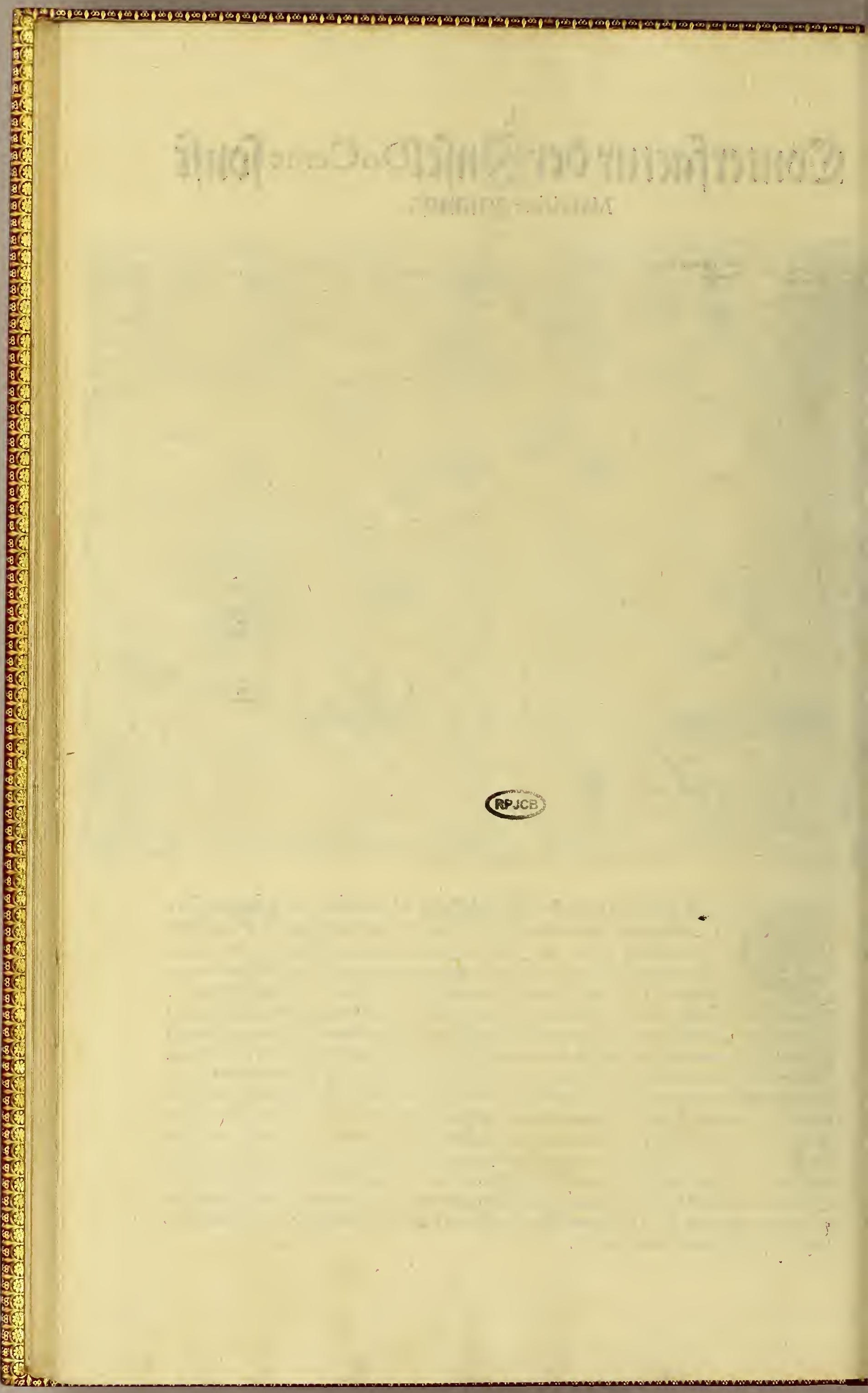


II.

Sllusecigung def ienigen fo die 居ol

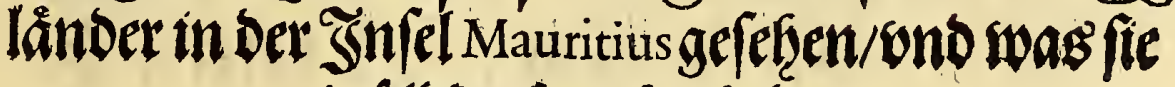
Dafelbftaufiggeriffitet ḩaben.

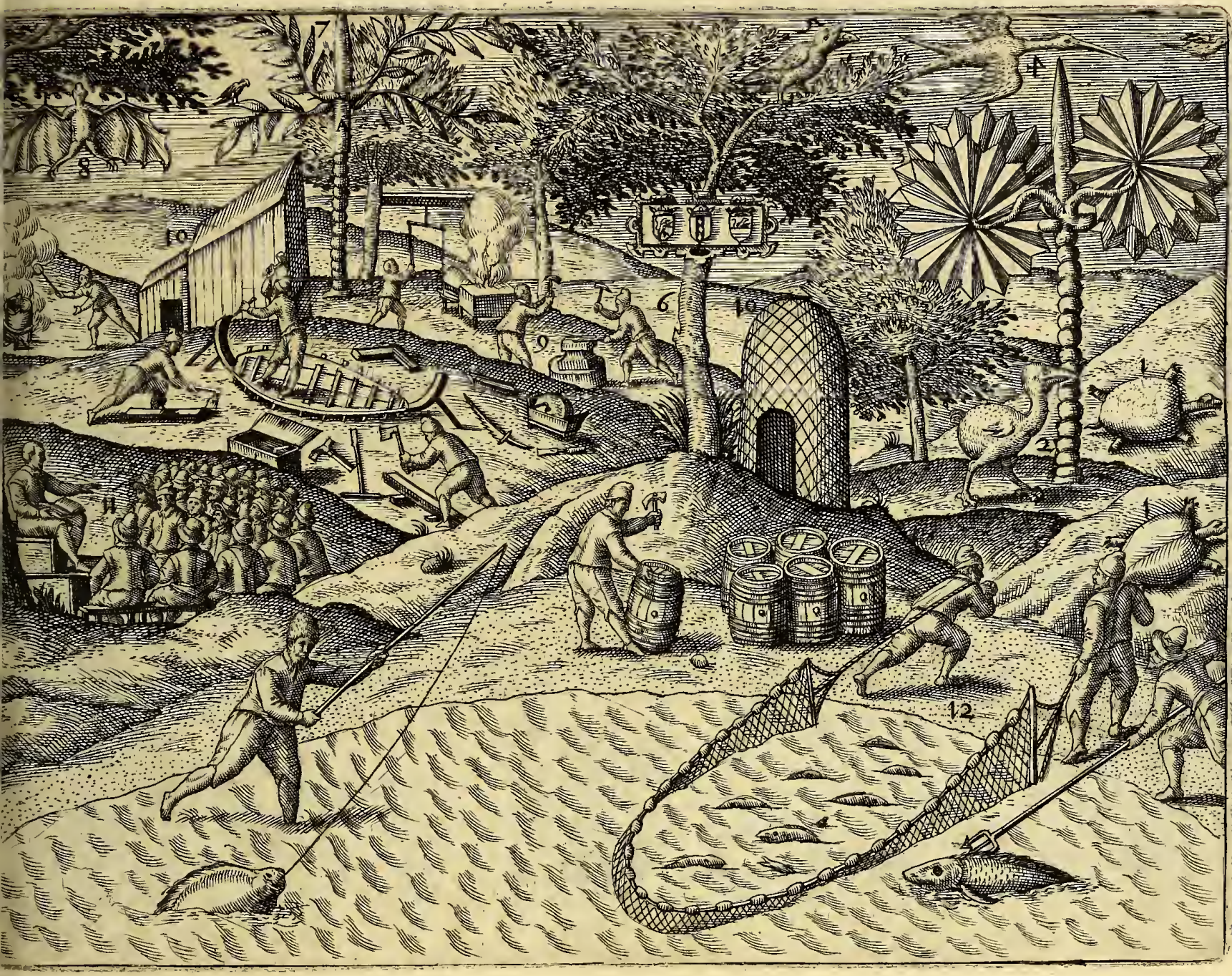

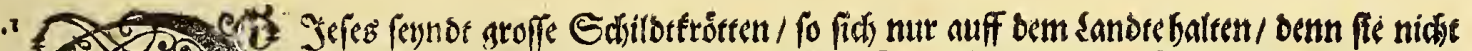

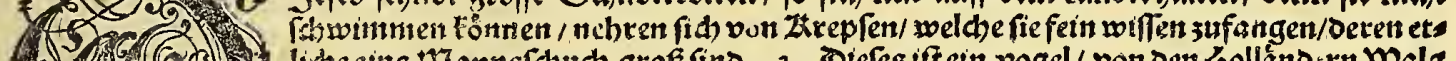

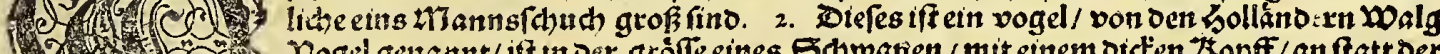

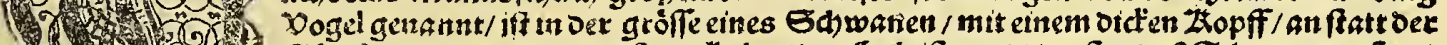

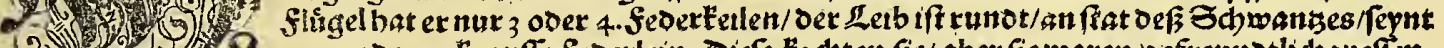

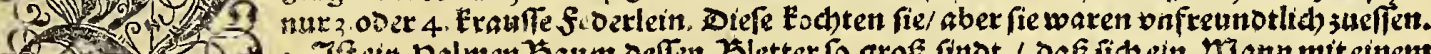

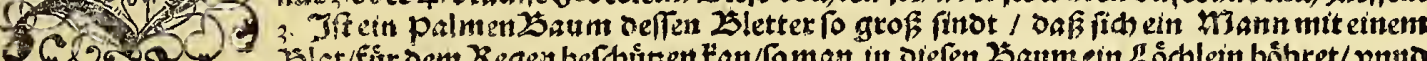

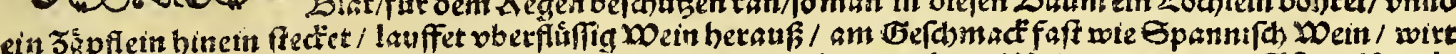

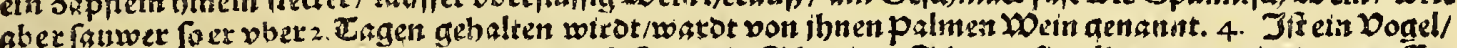

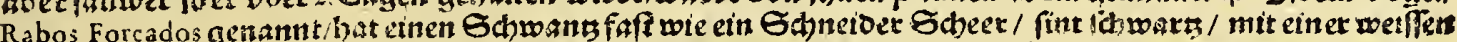

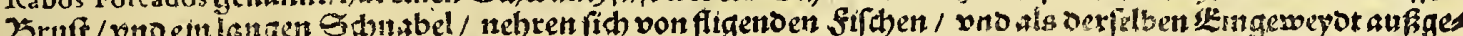

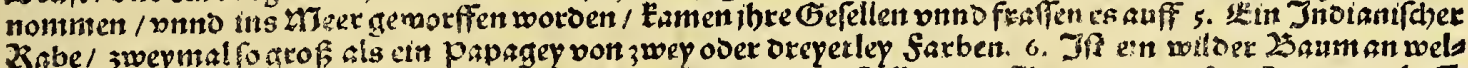

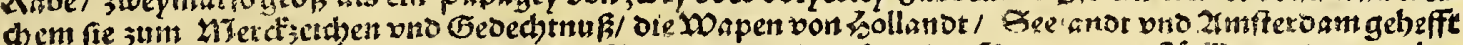

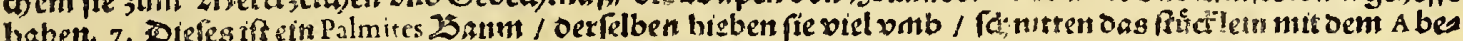

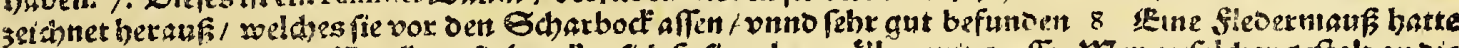

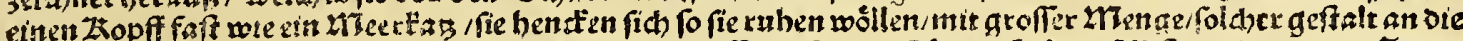

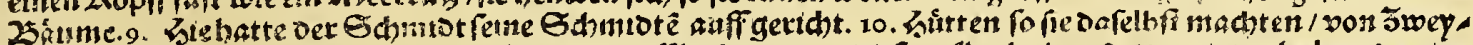

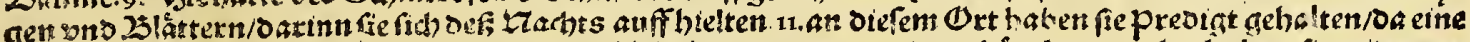

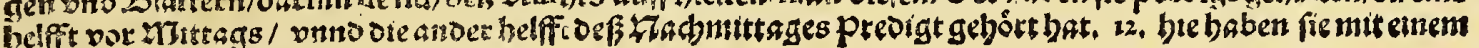

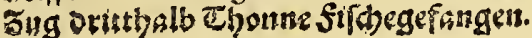




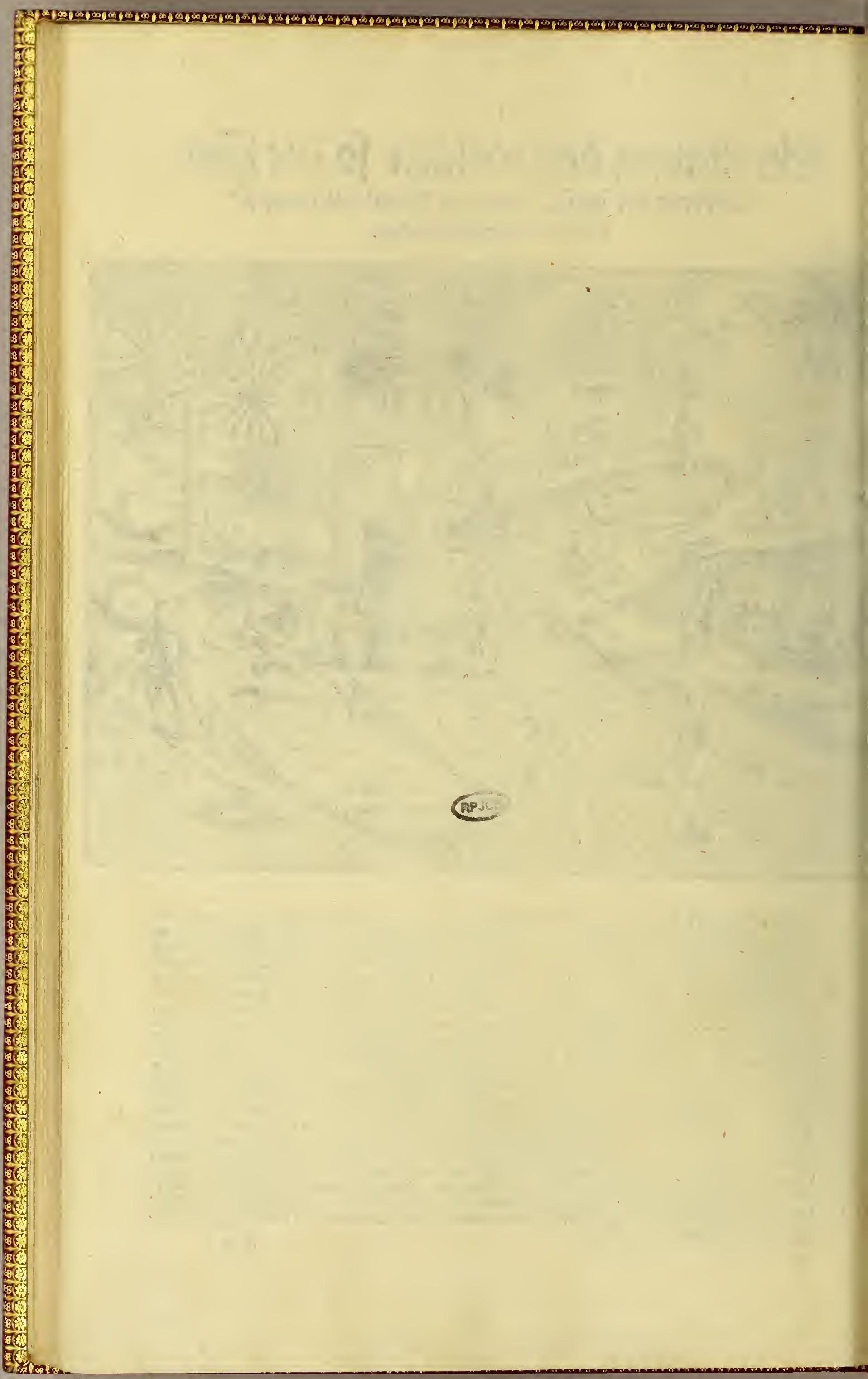




\section{III. Tัง 孔̆ Tuban.}

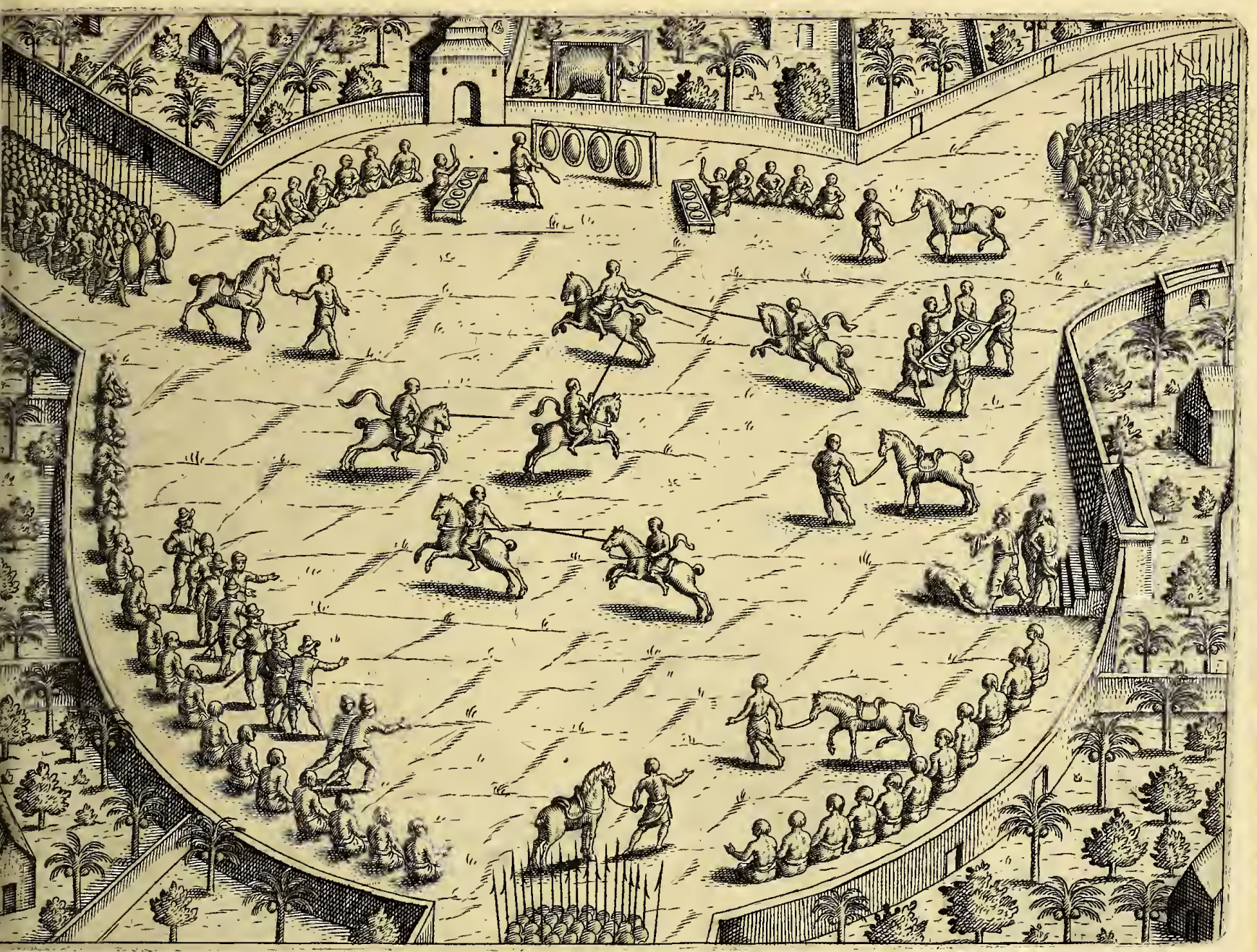

\footnotetext{
(5) SEfte onter andern / Den 23. Sanuarijoeß IS99.

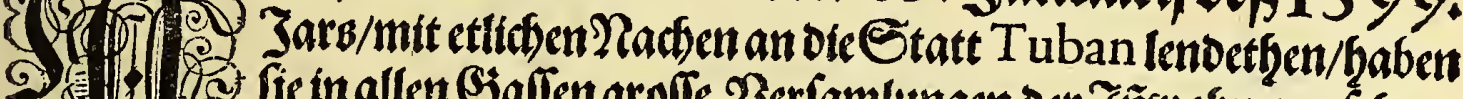

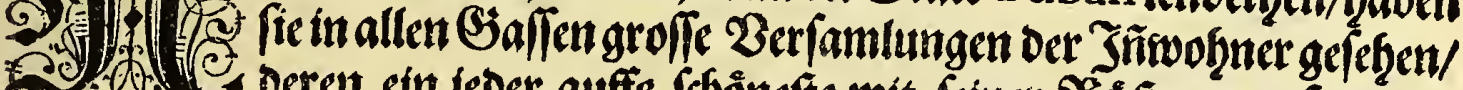

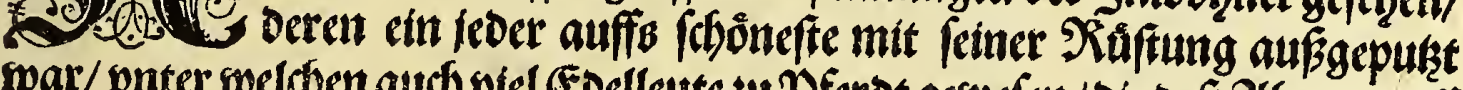

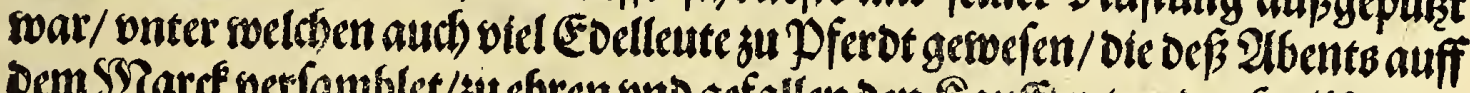

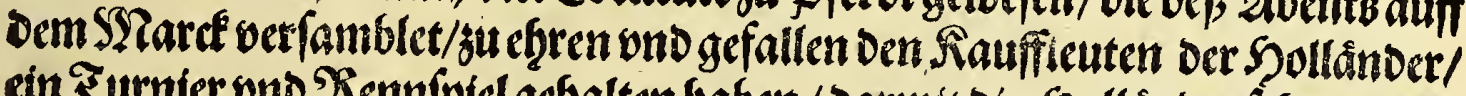

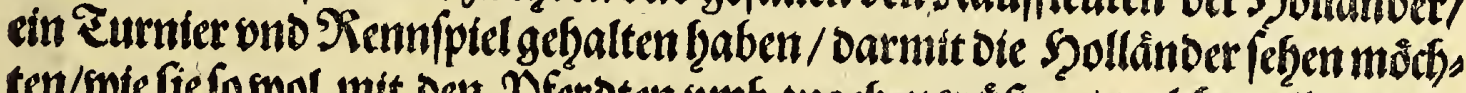

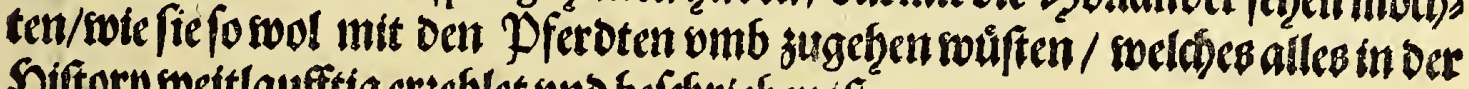
Siftory weitlauffitg erstęlet wnd beforticbenift.
} 


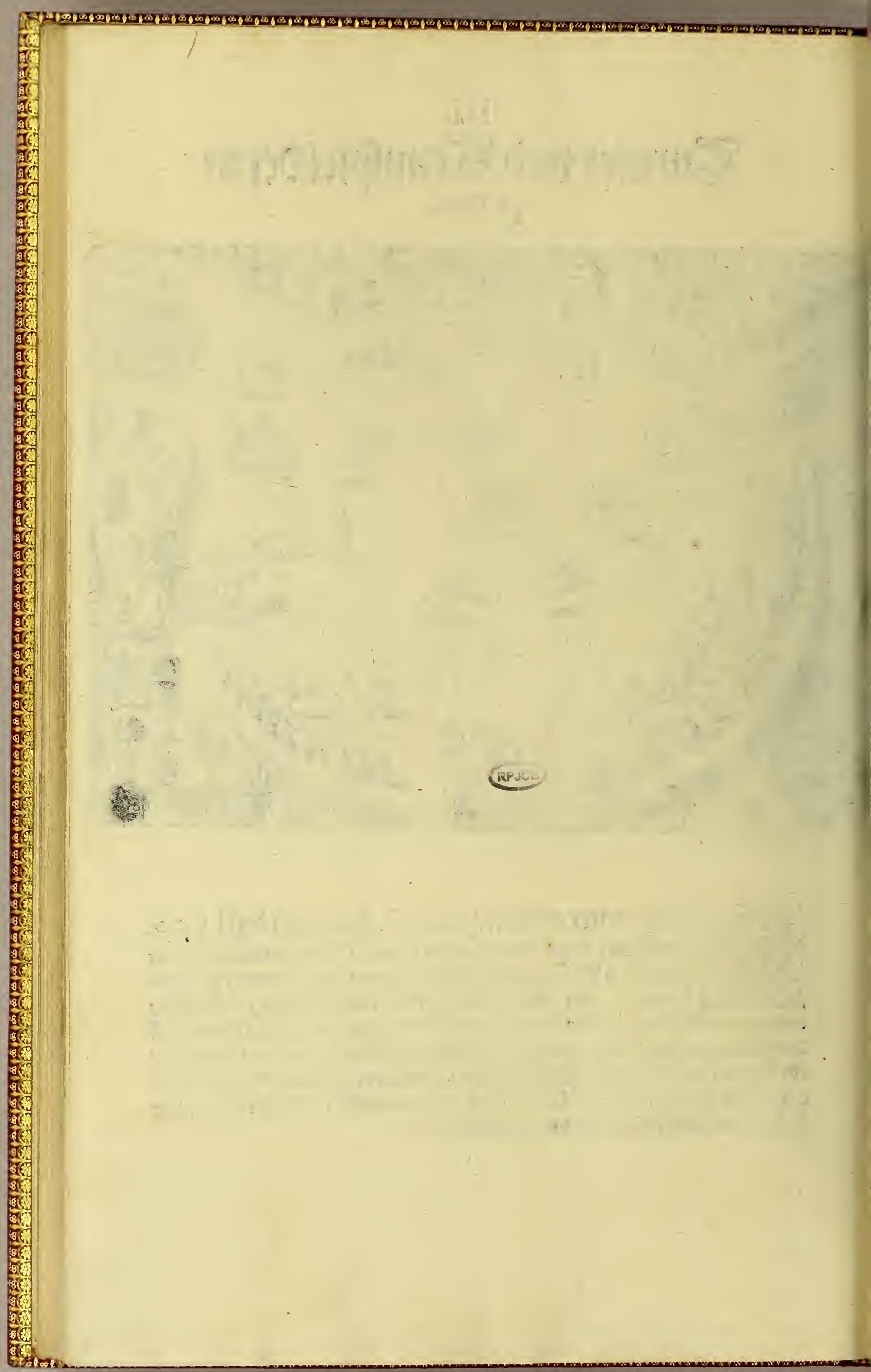


IV.

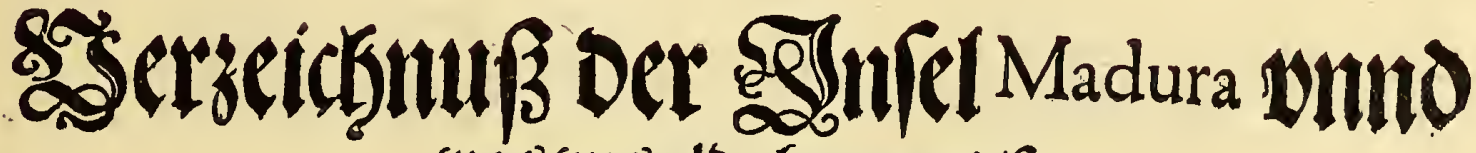
wasons albabegegnet if

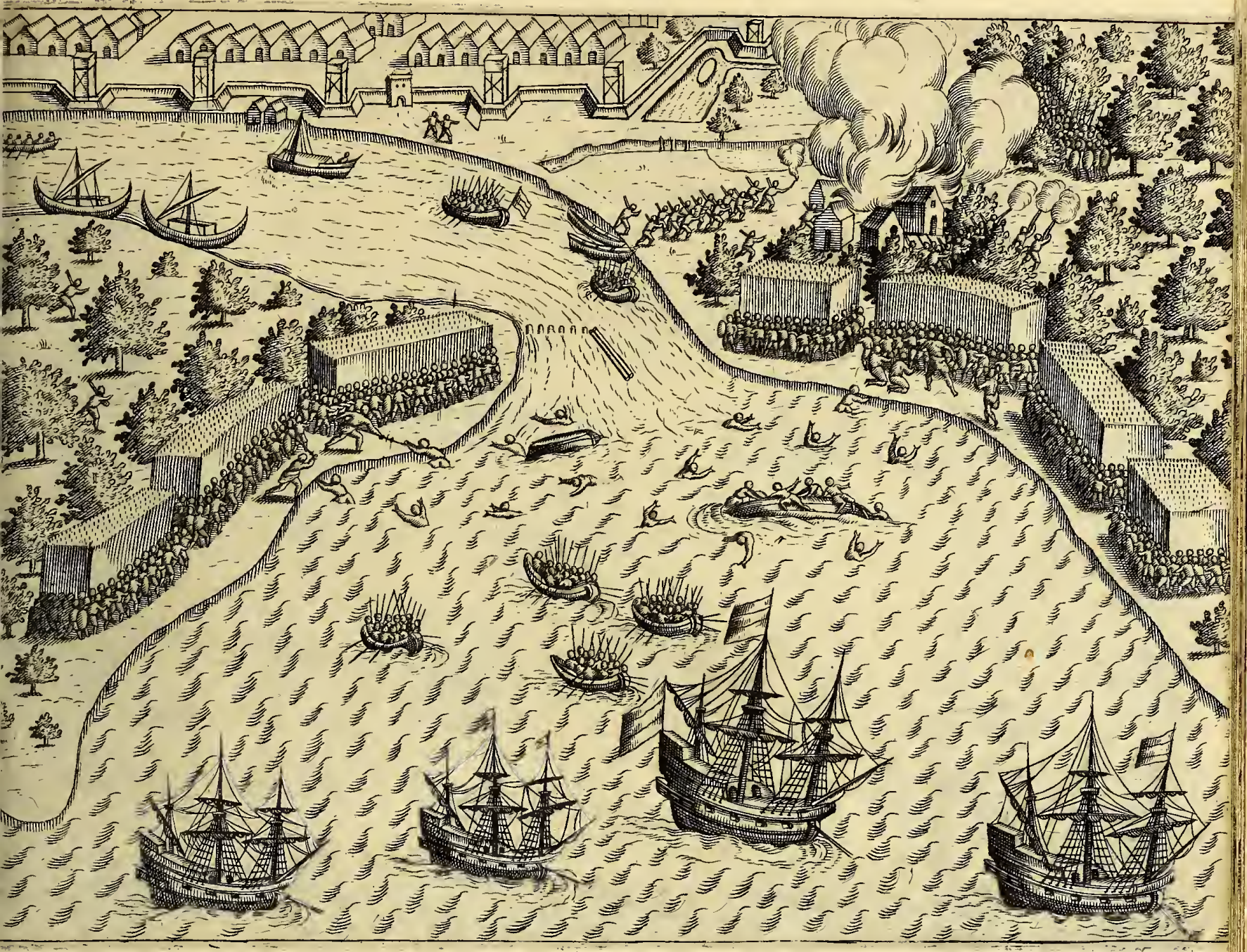

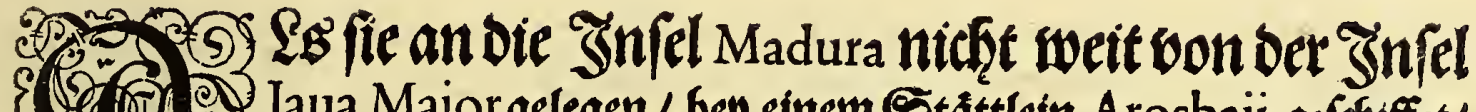

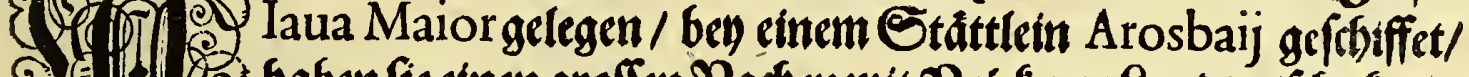

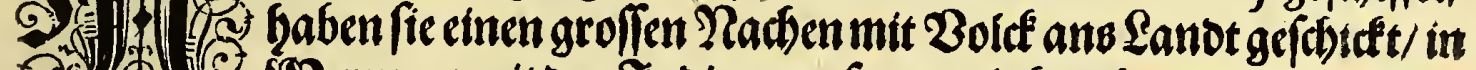

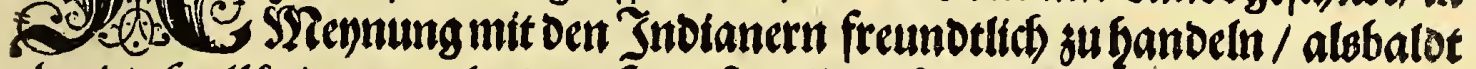
aber Die Syollânoer angelendet / findt fie alle gefângitich eingesogen worden/

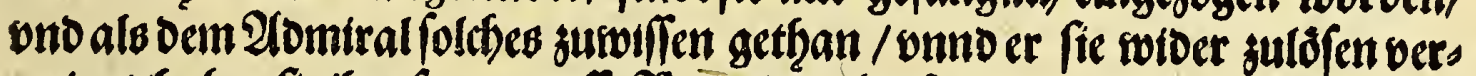

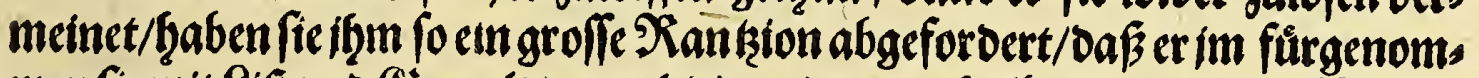

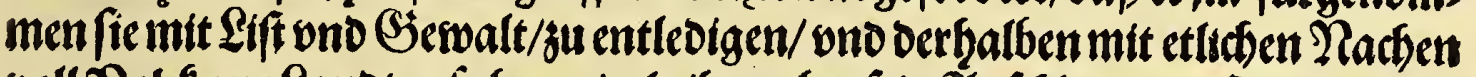

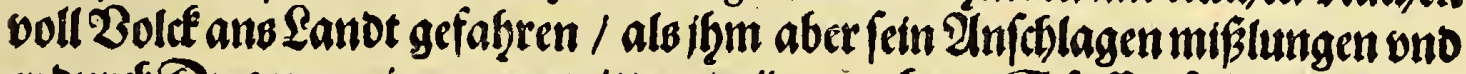

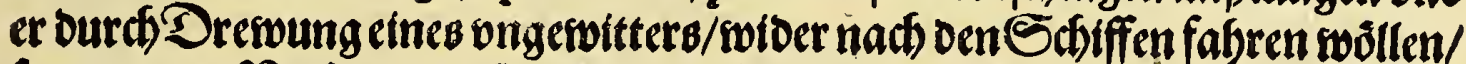

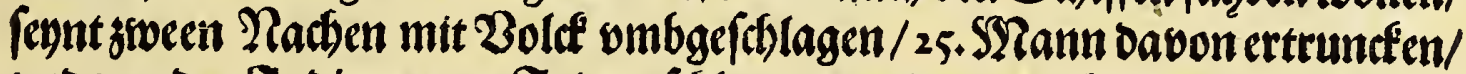

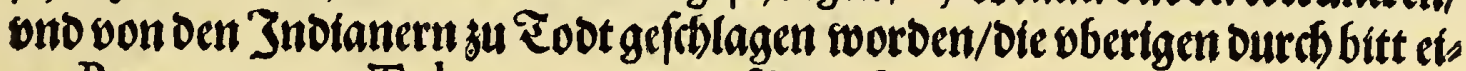

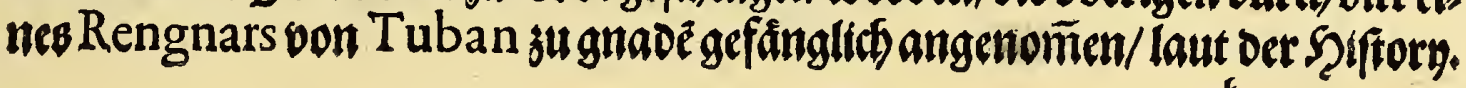




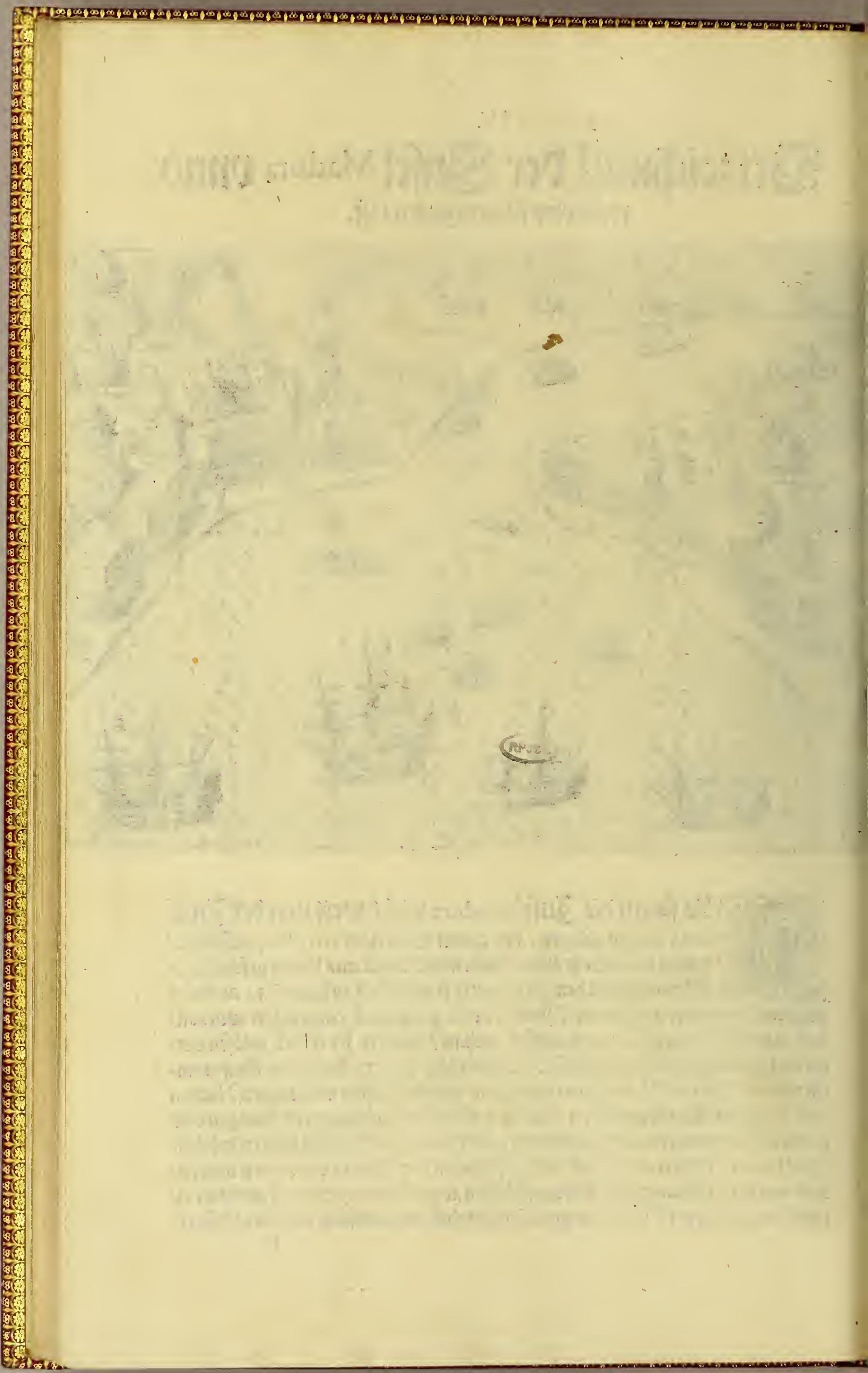




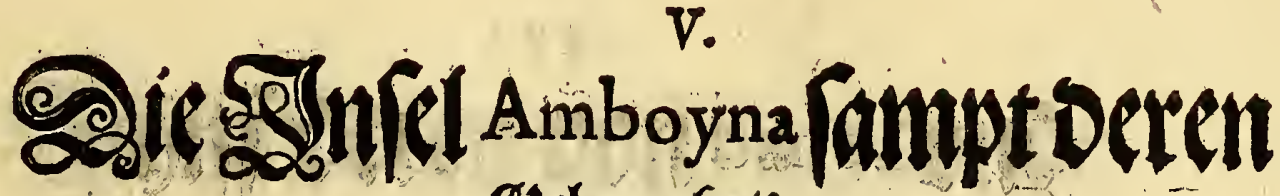 Bfelegentert:}
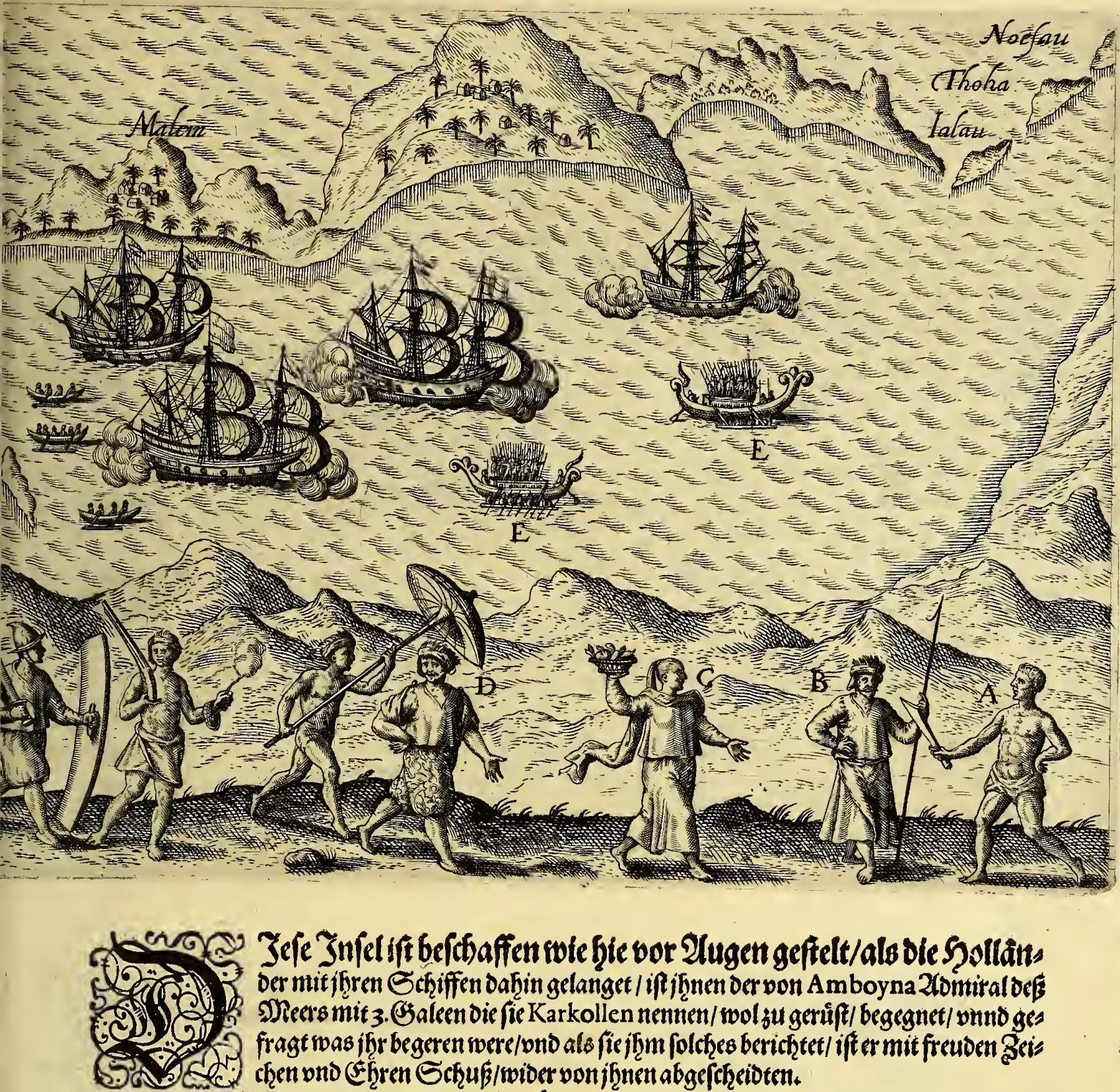

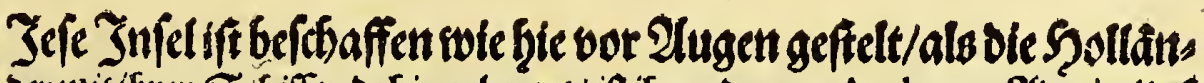

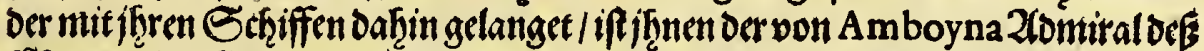

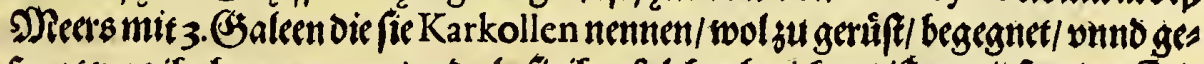

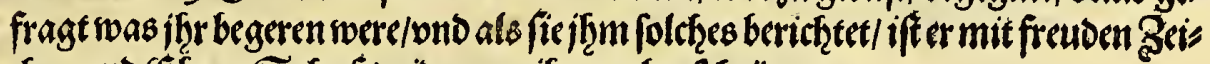

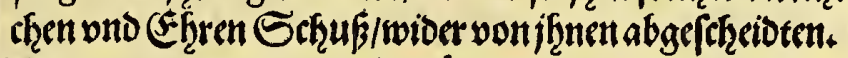

Ulbbiloung beten von A mboyna mit A.B.C.verseifonet.

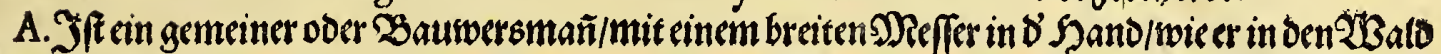

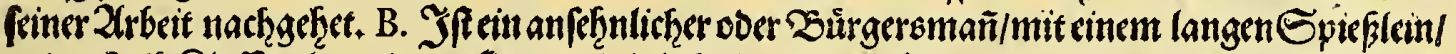

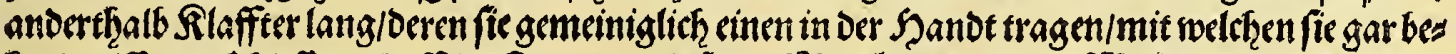

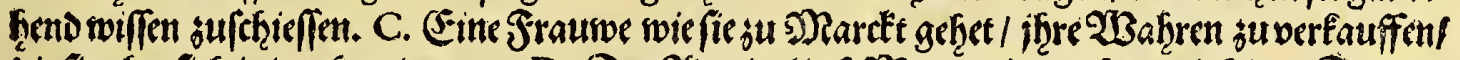

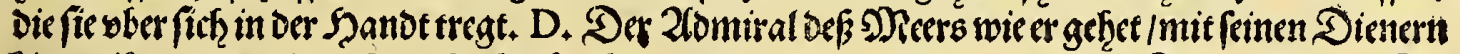

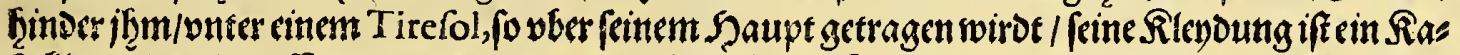

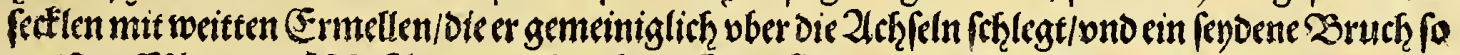

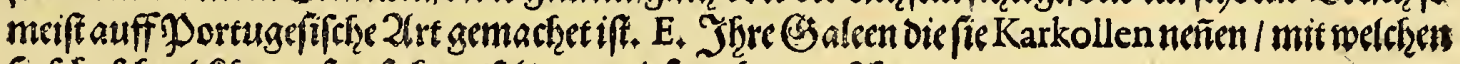

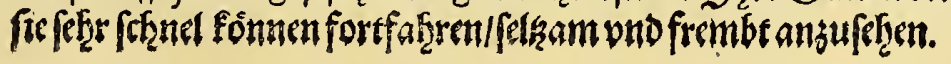




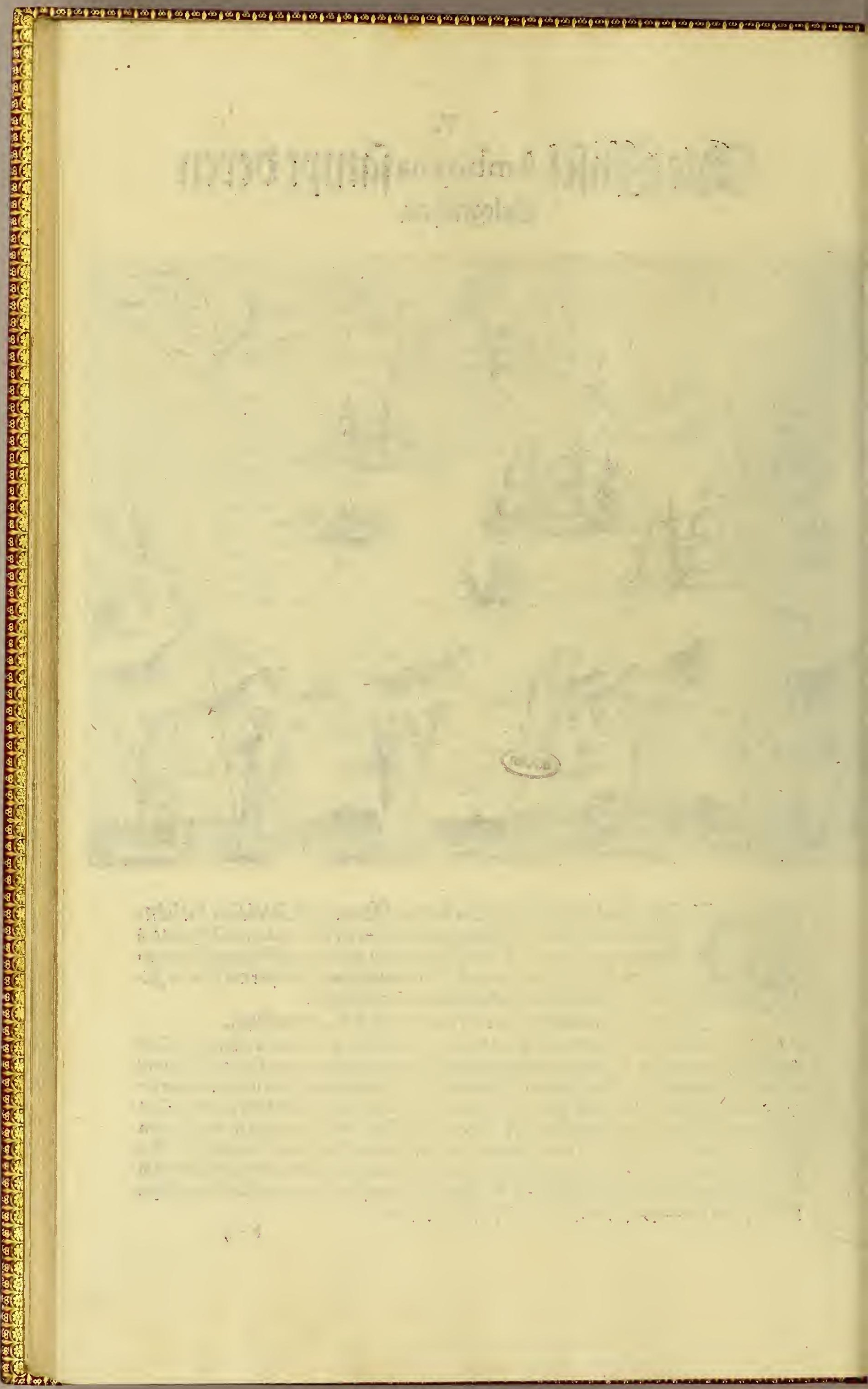




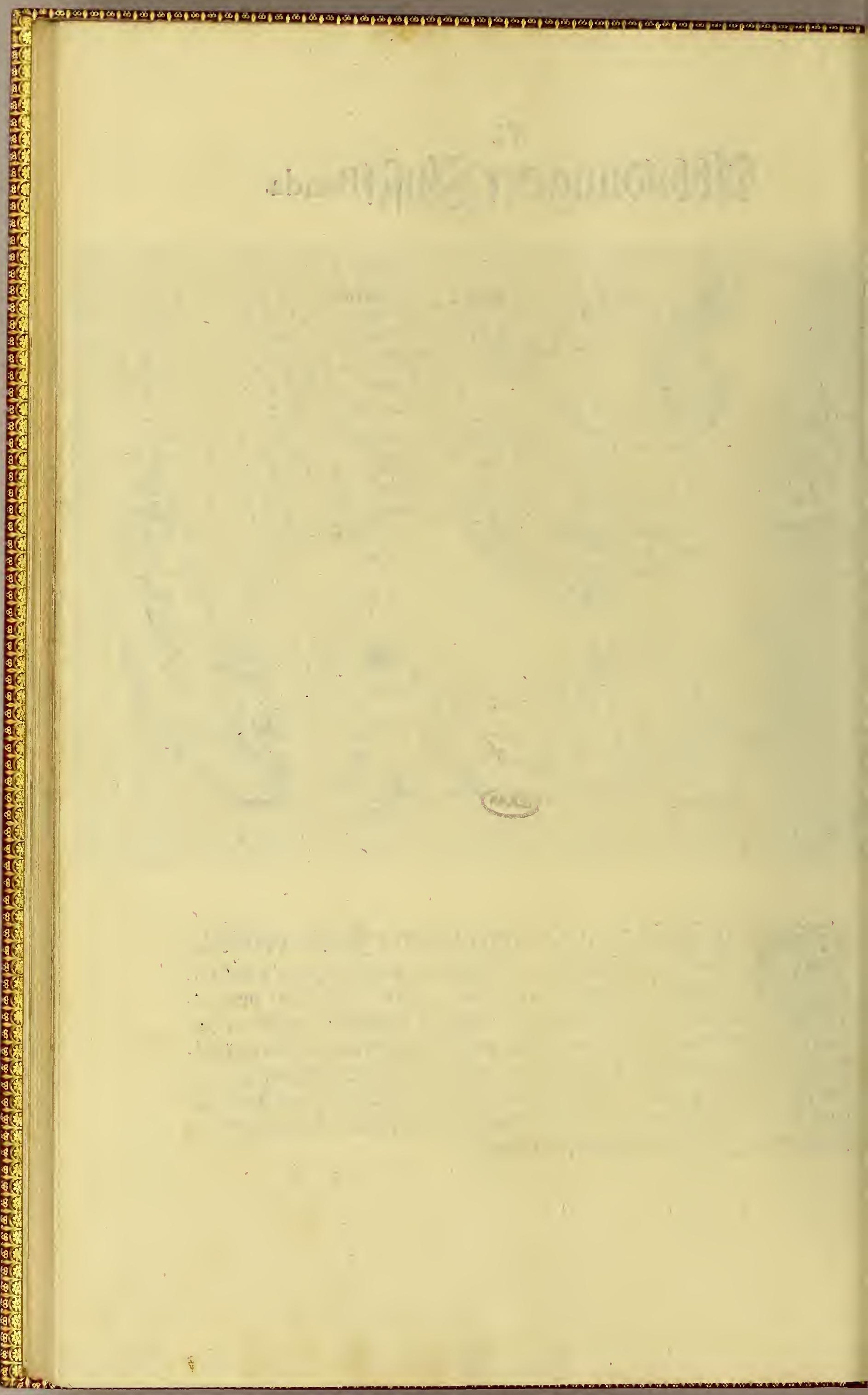




\section{Sriéc die von Nera den Jäbllandorichen Bicestomital empfangen fiaben.}

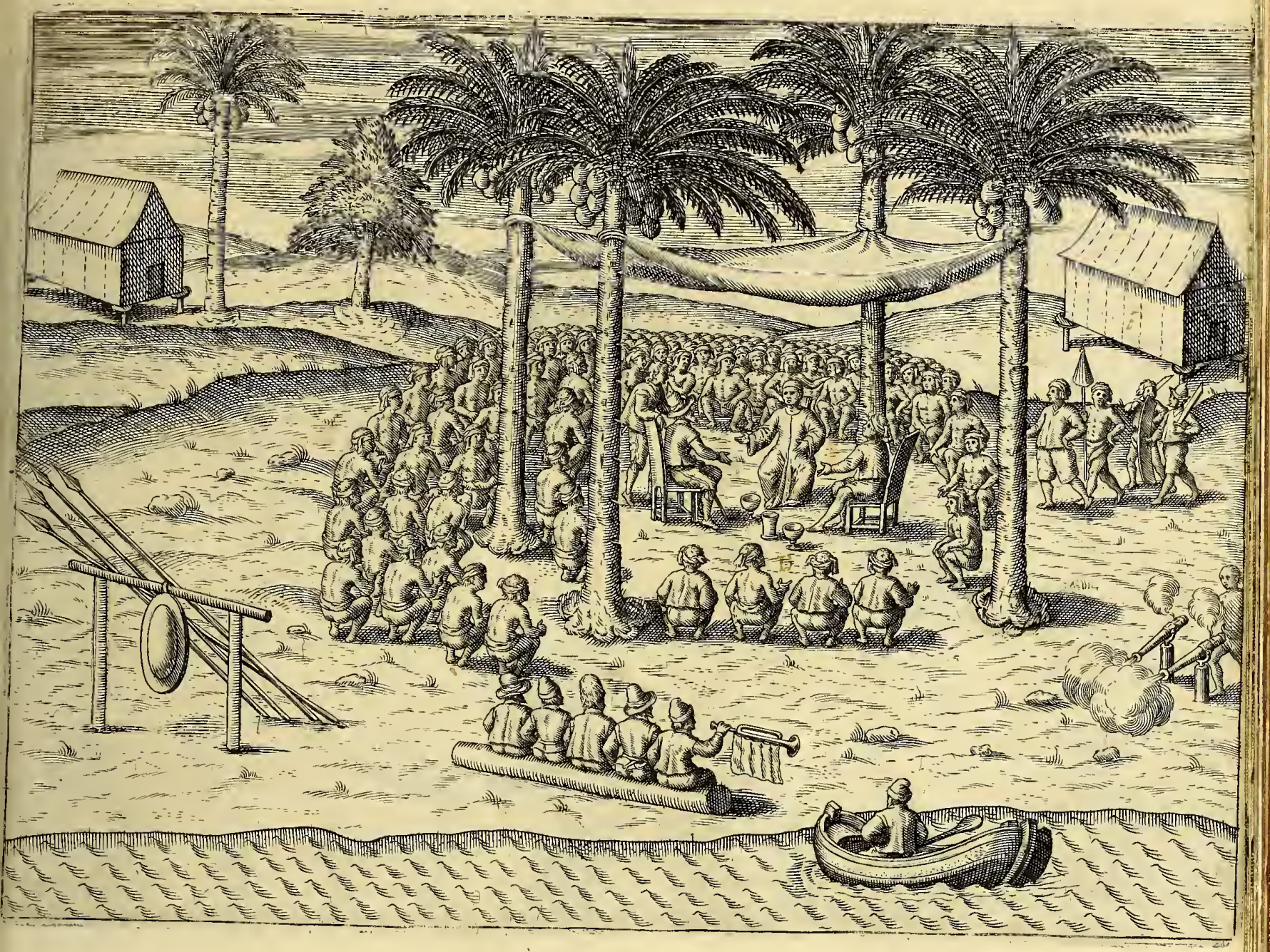

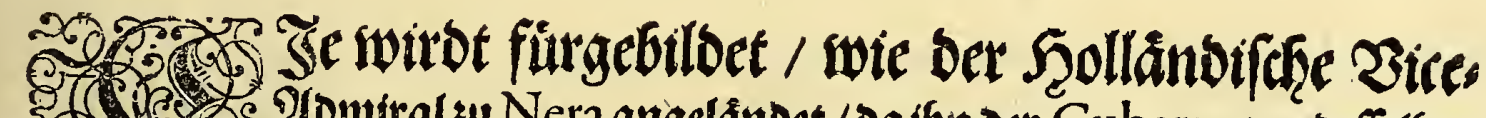

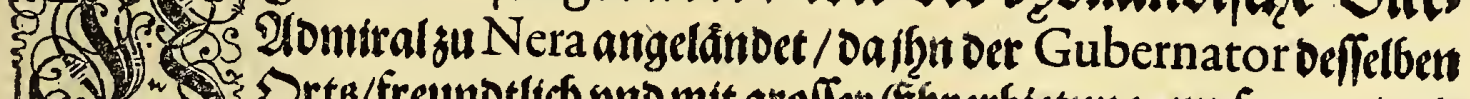

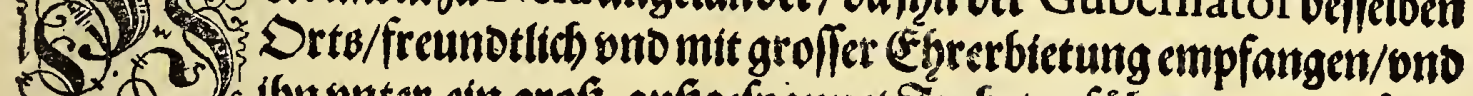

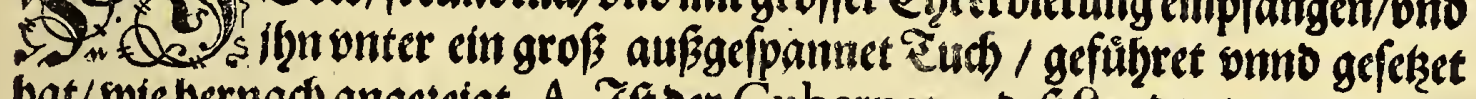

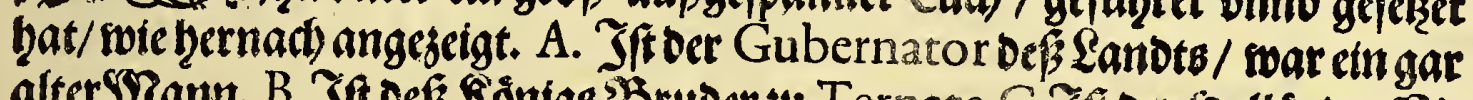

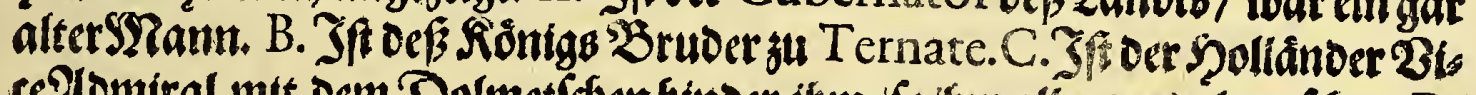

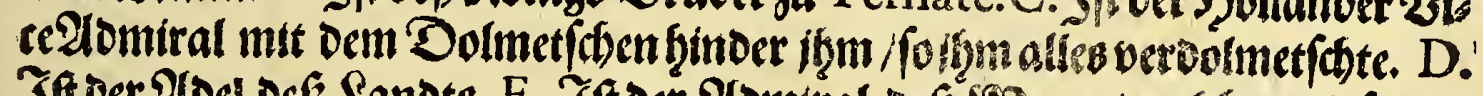

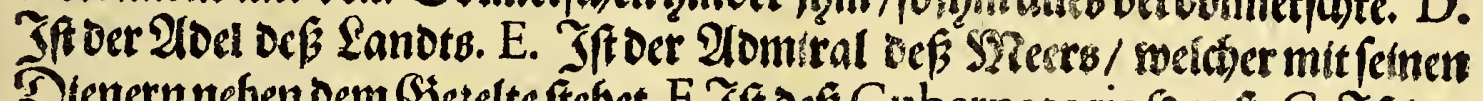

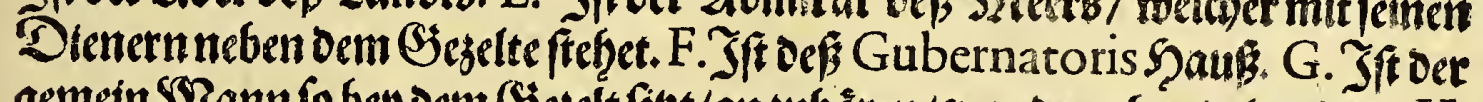

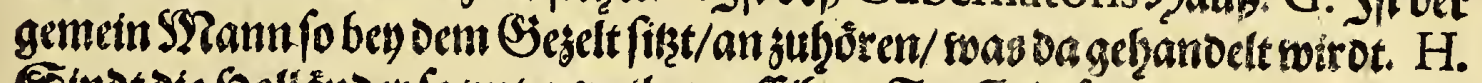

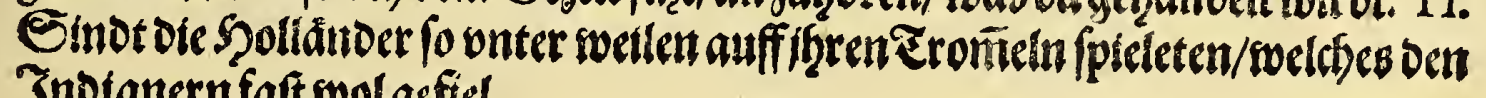
Indianern faft wool gefiel. 


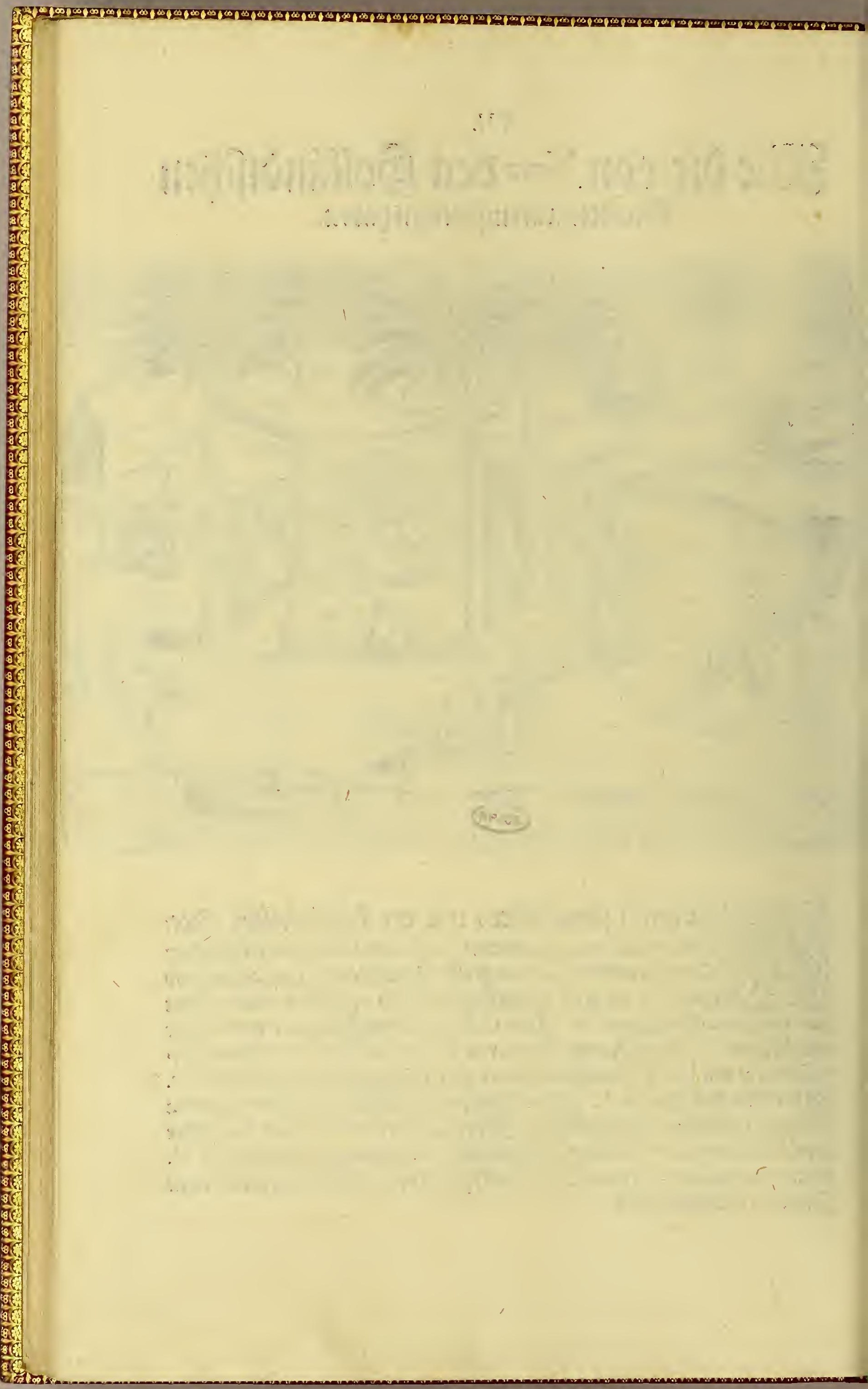




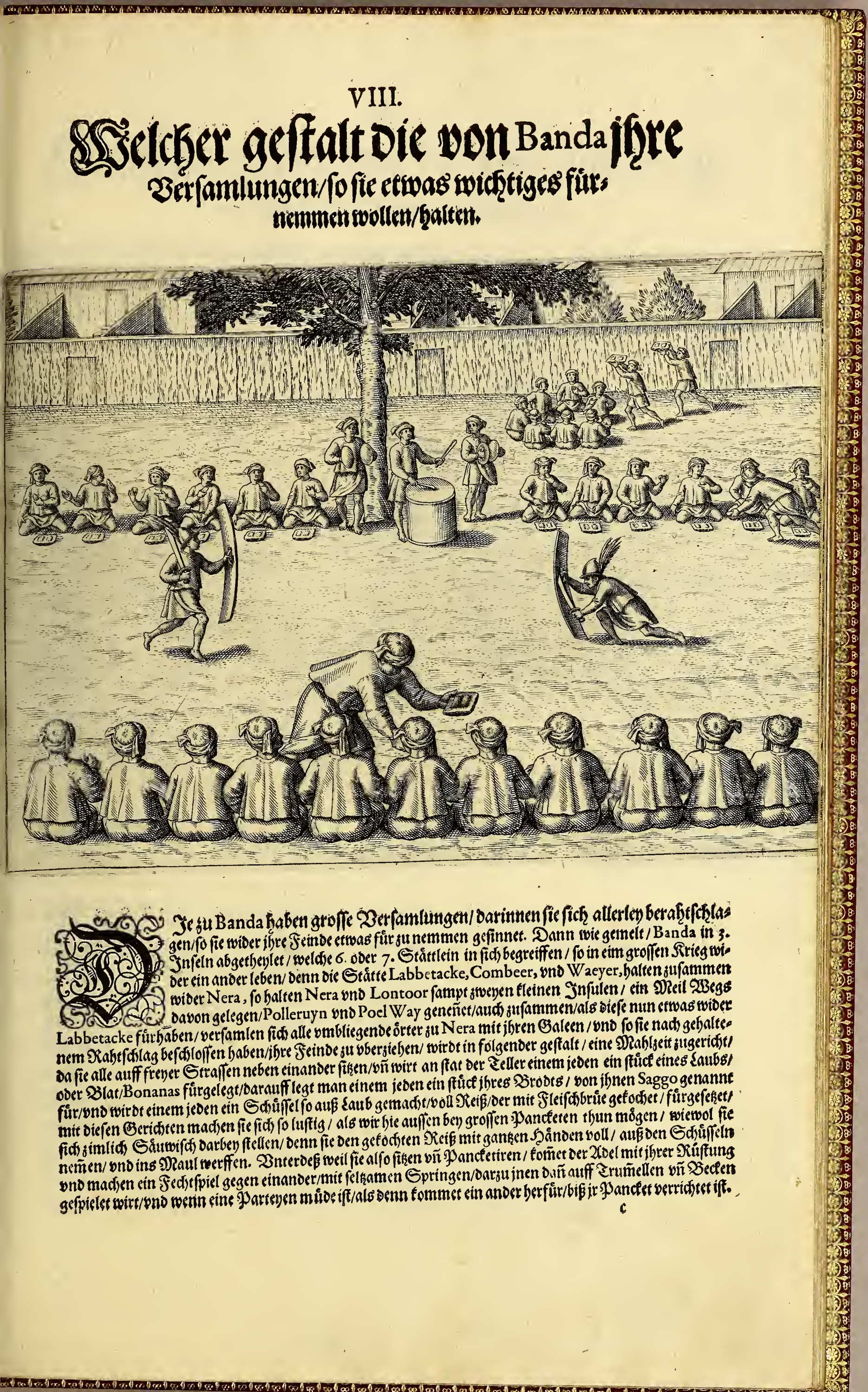




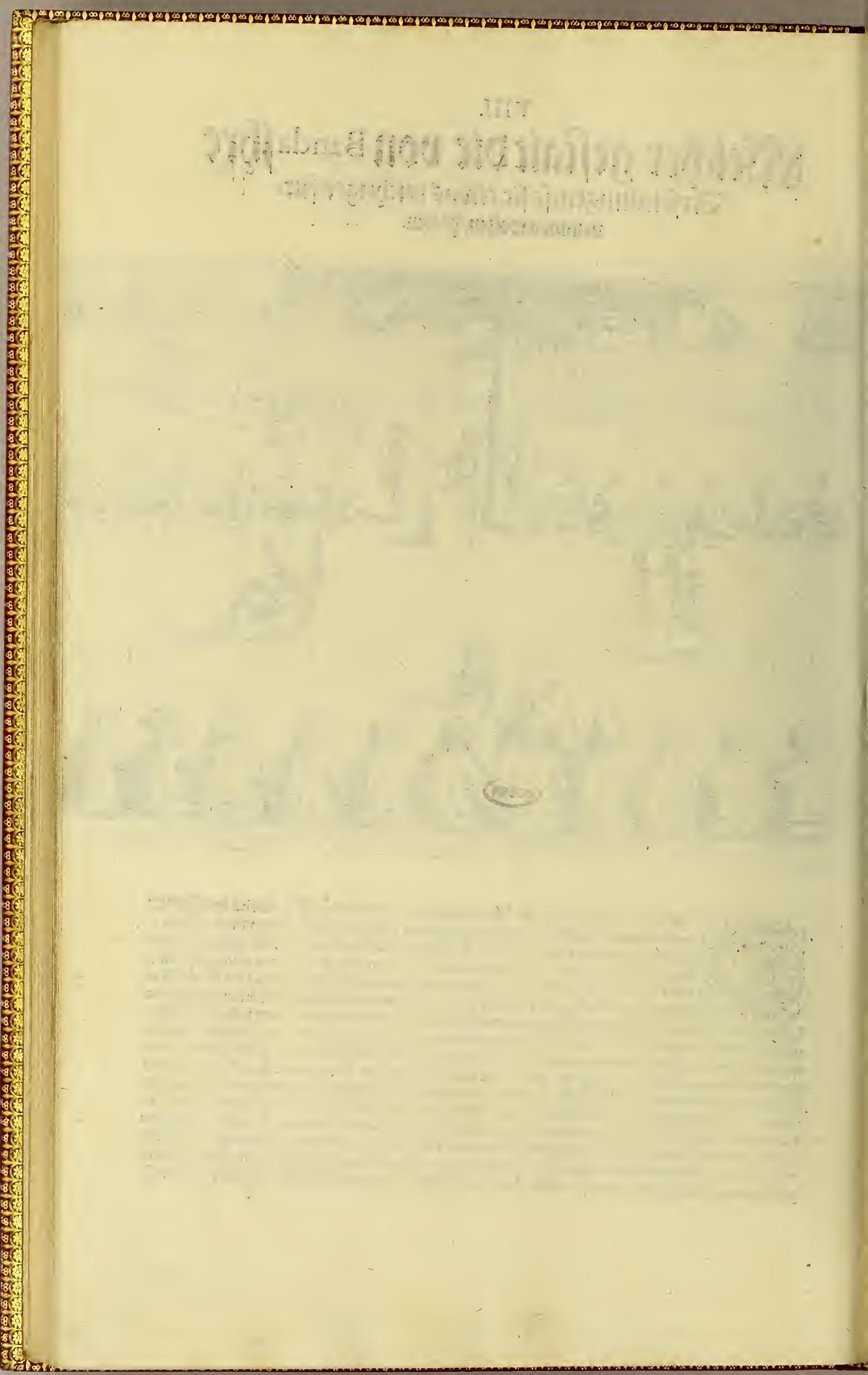




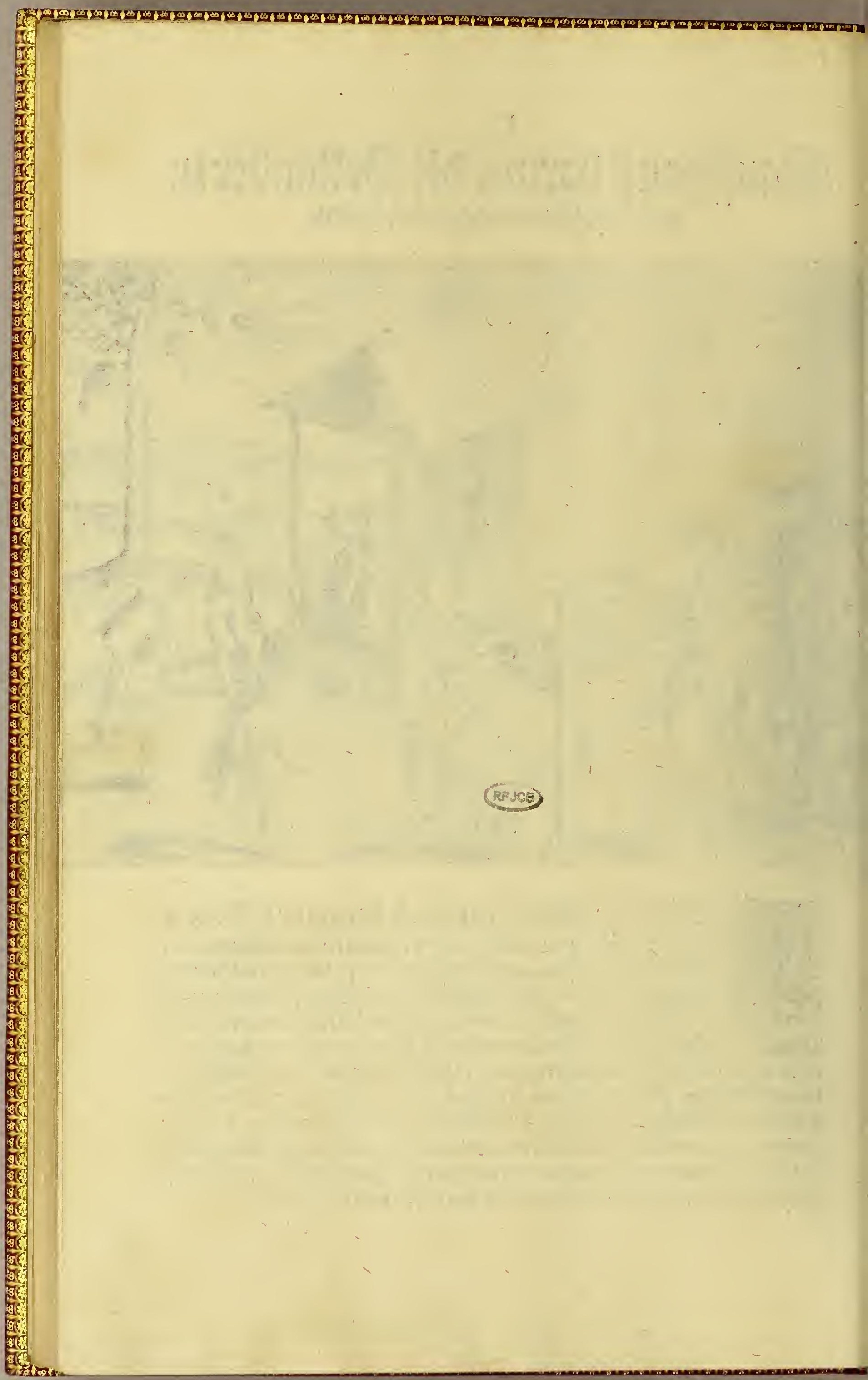




\section{X. \\ Singentliche offtirfilloung etlicher Perfonen zuBanda.}

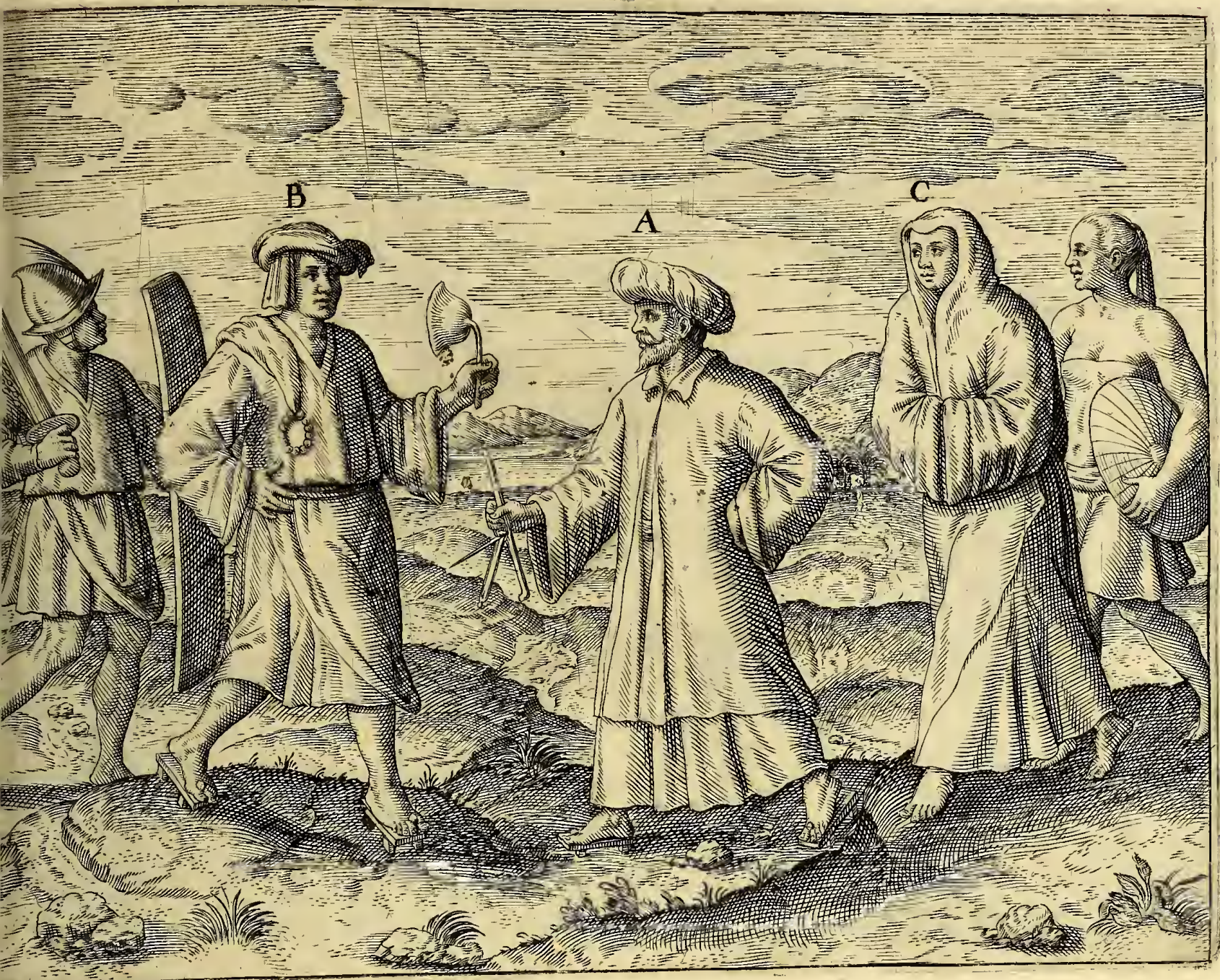

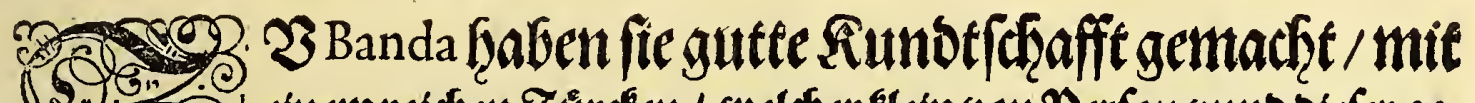

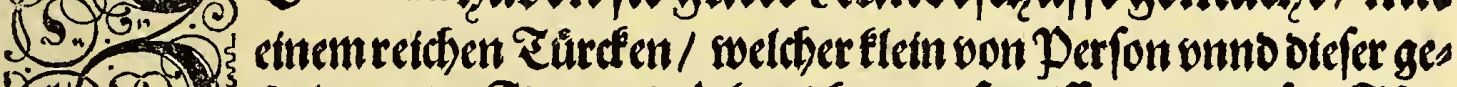

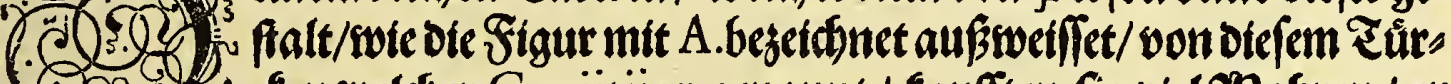

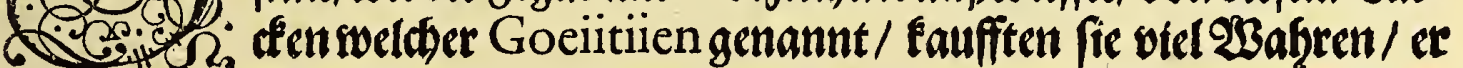

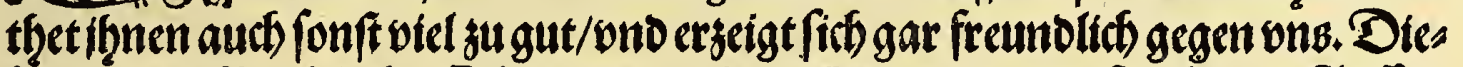

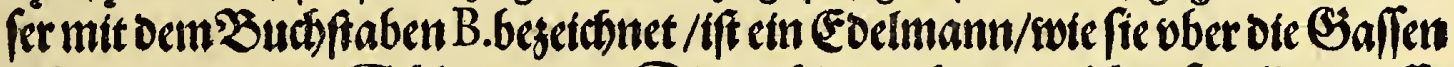

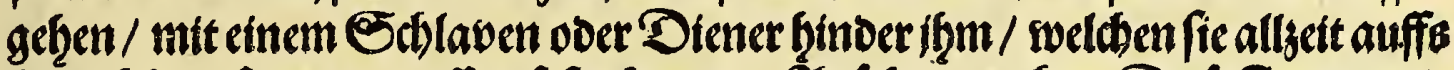

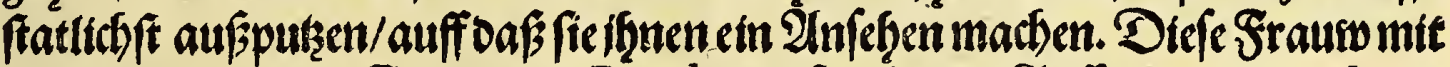

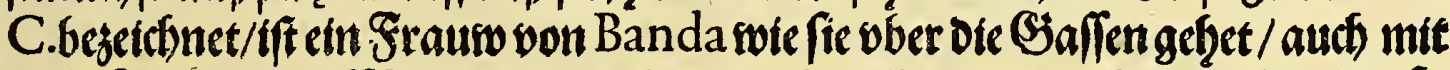

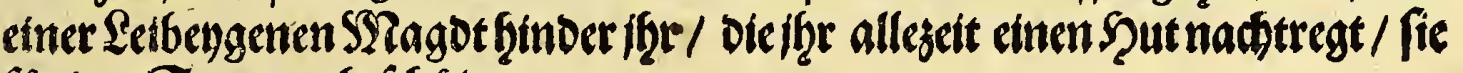
für Der Sonne jubefturken. 


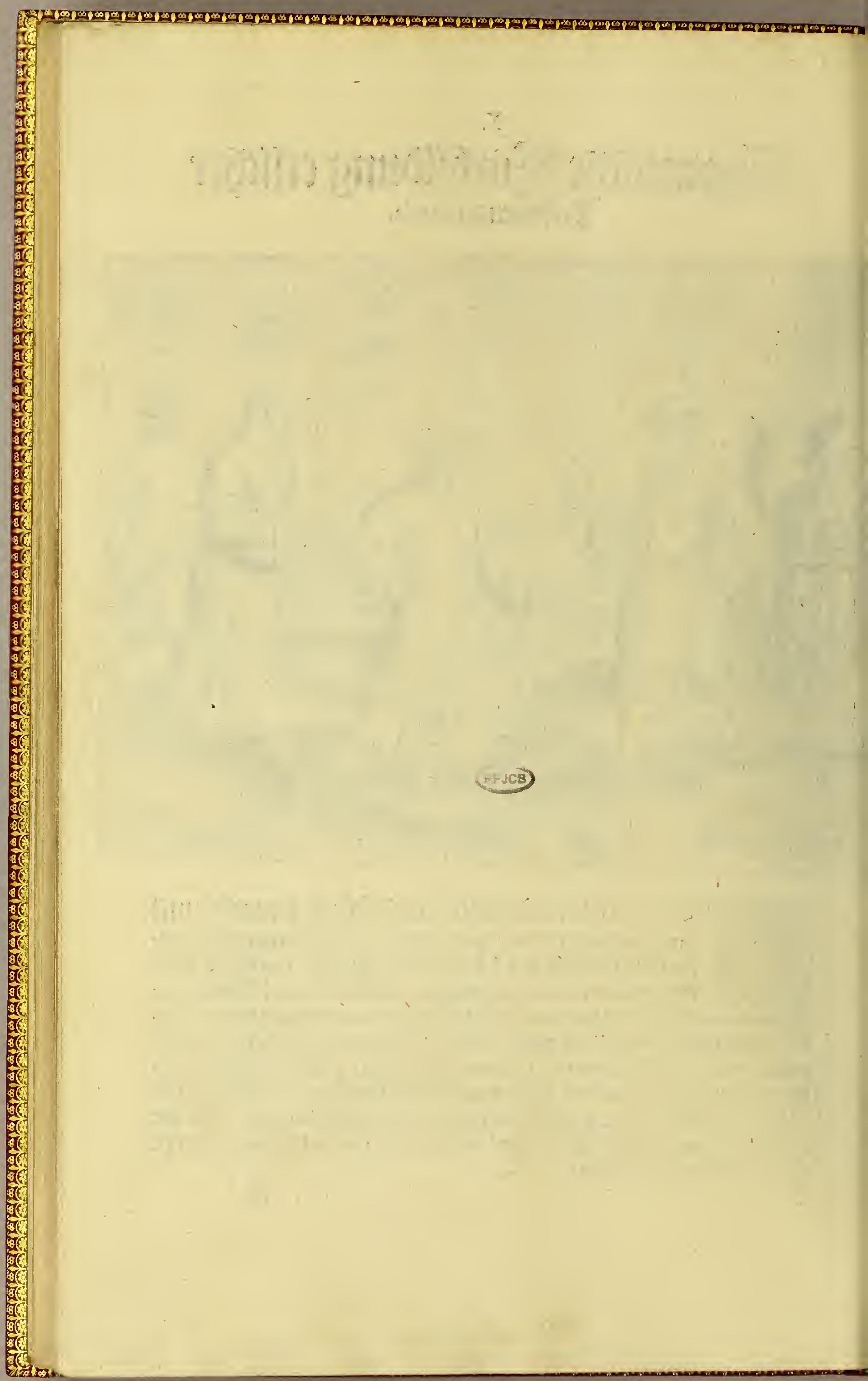




\section{$\mathrm{Xl}$.

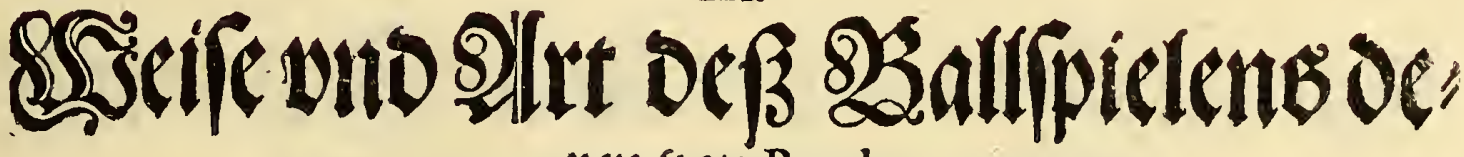

ren won Banda.

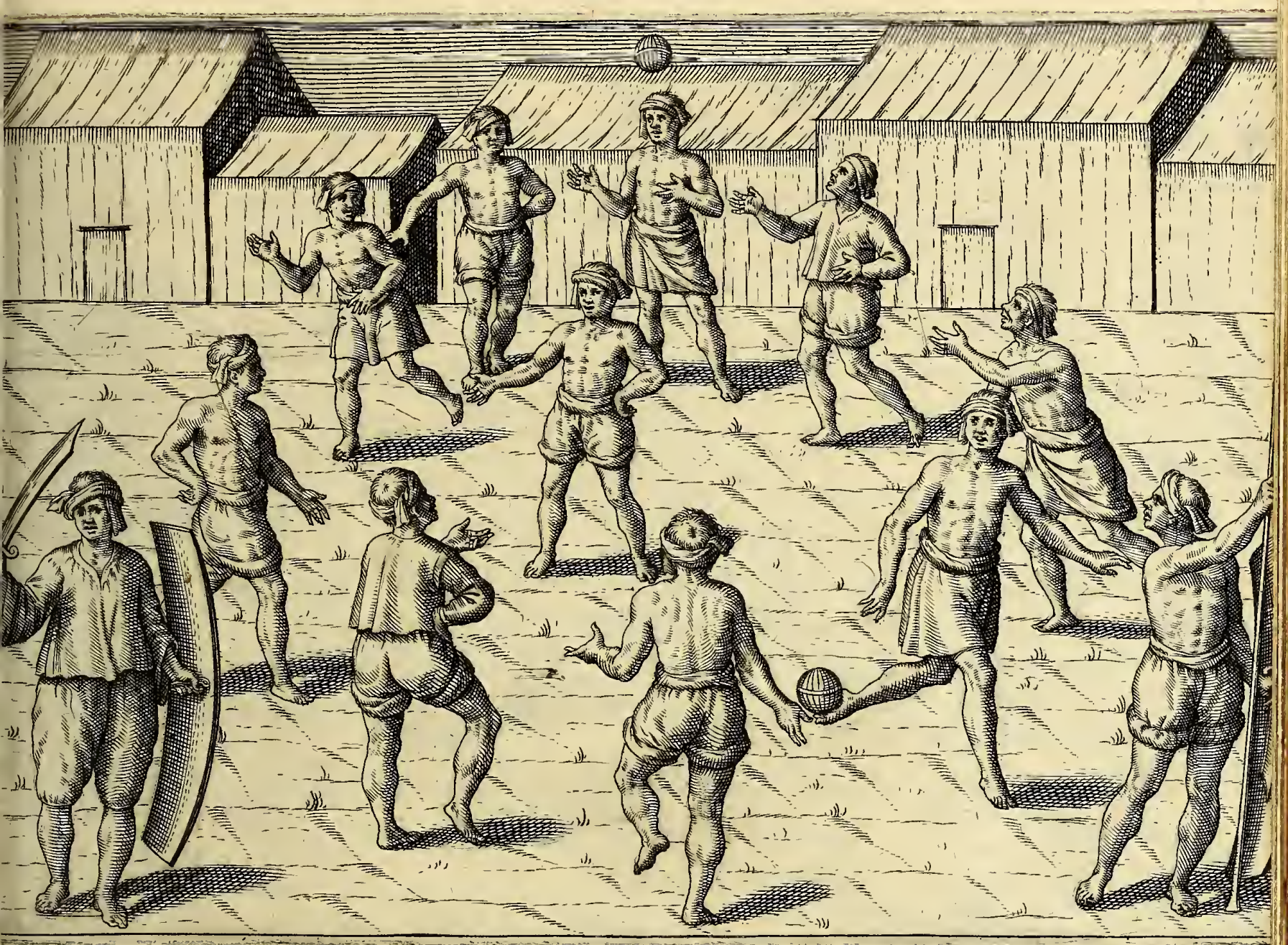

eno. Yevon Bandafpielen folgenoer geftalt mit bem Ballen/eg fies

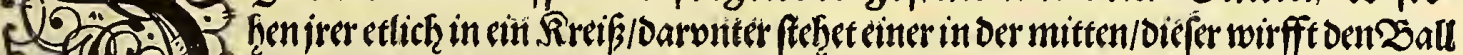

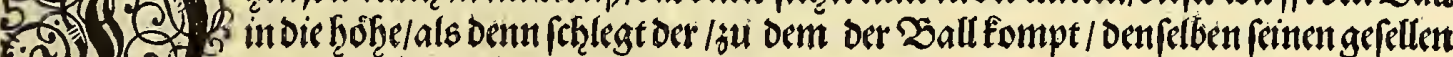

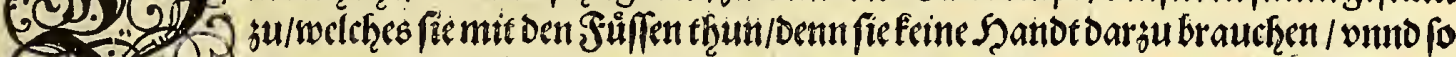

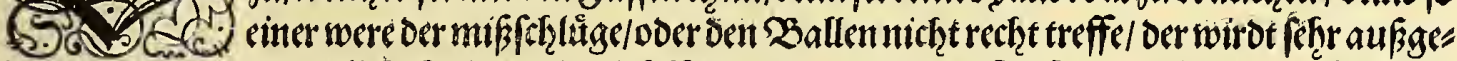

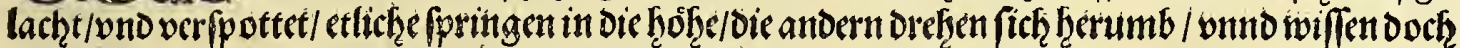

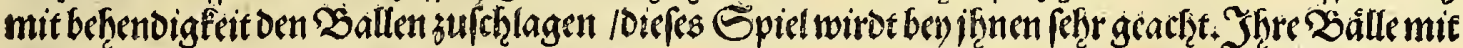

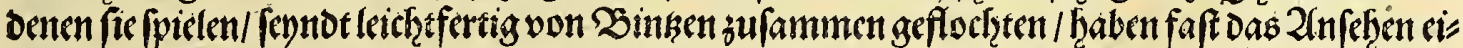

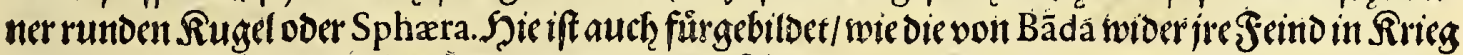

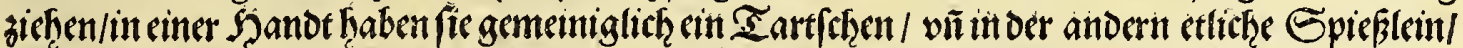

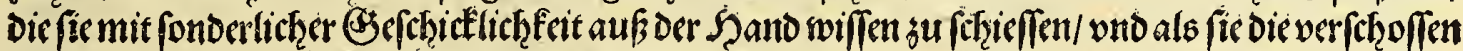

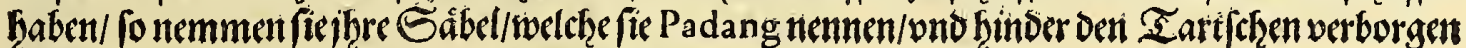
halten/mit denen thum fie einander groffen Scḩaben. 


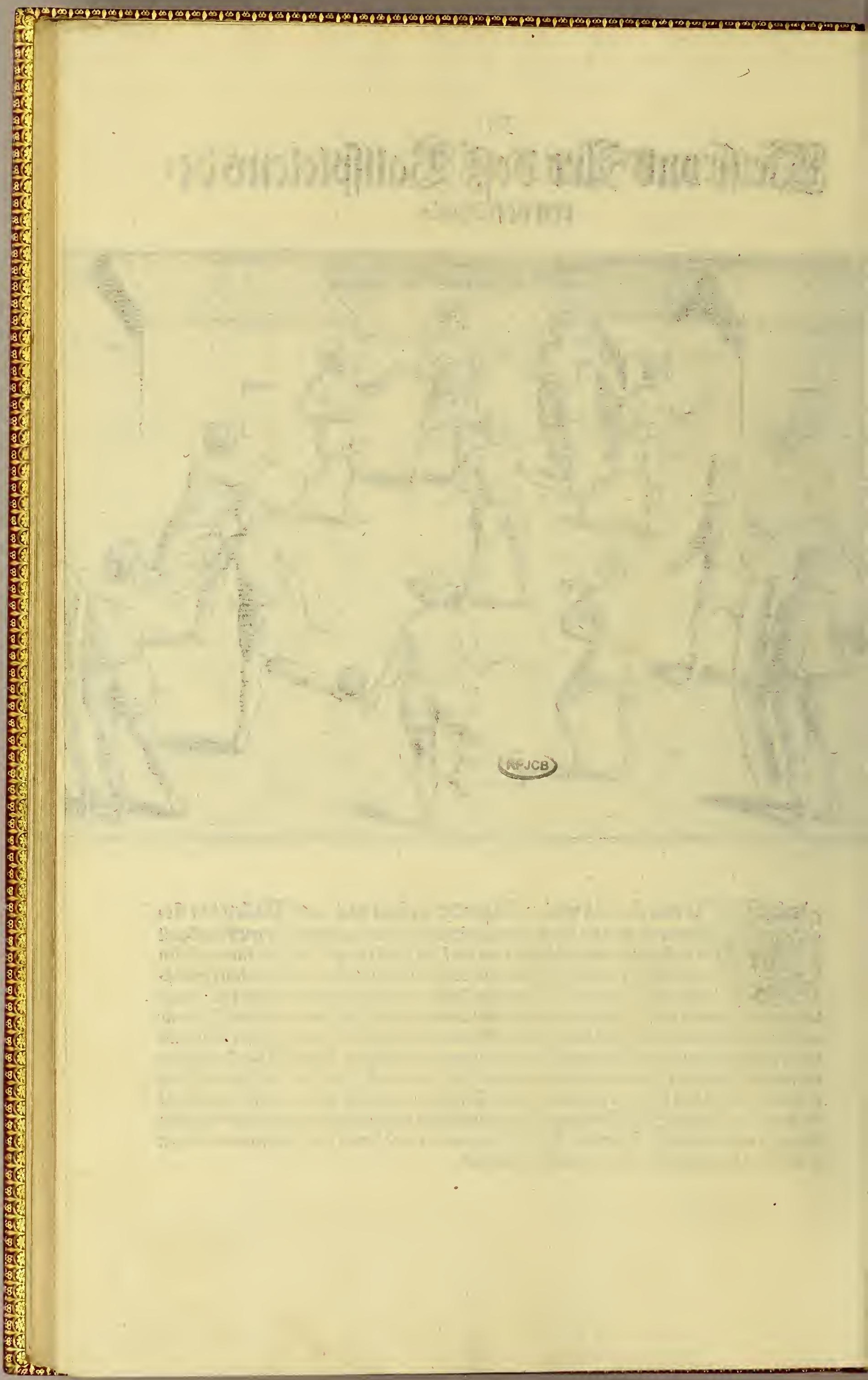




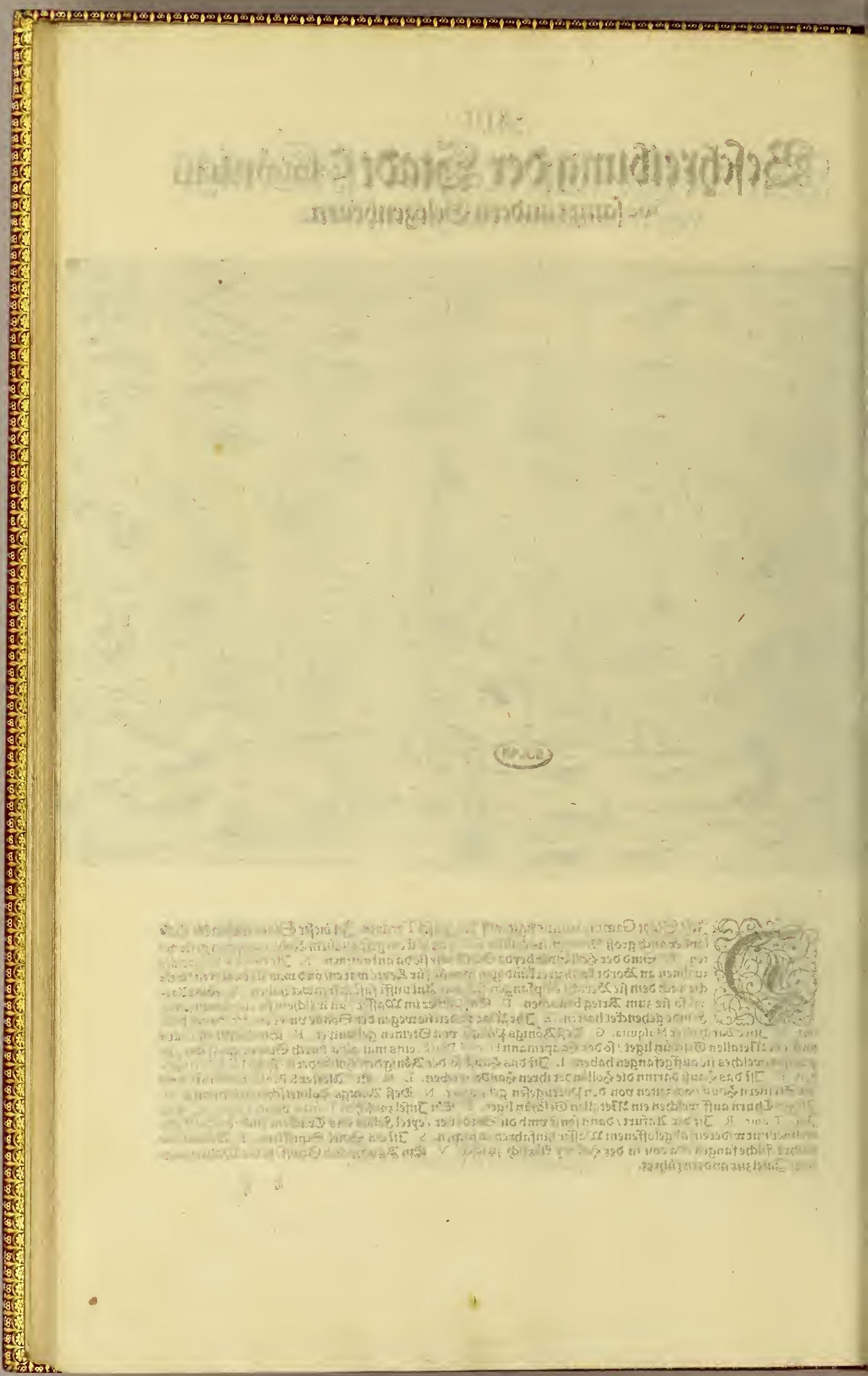




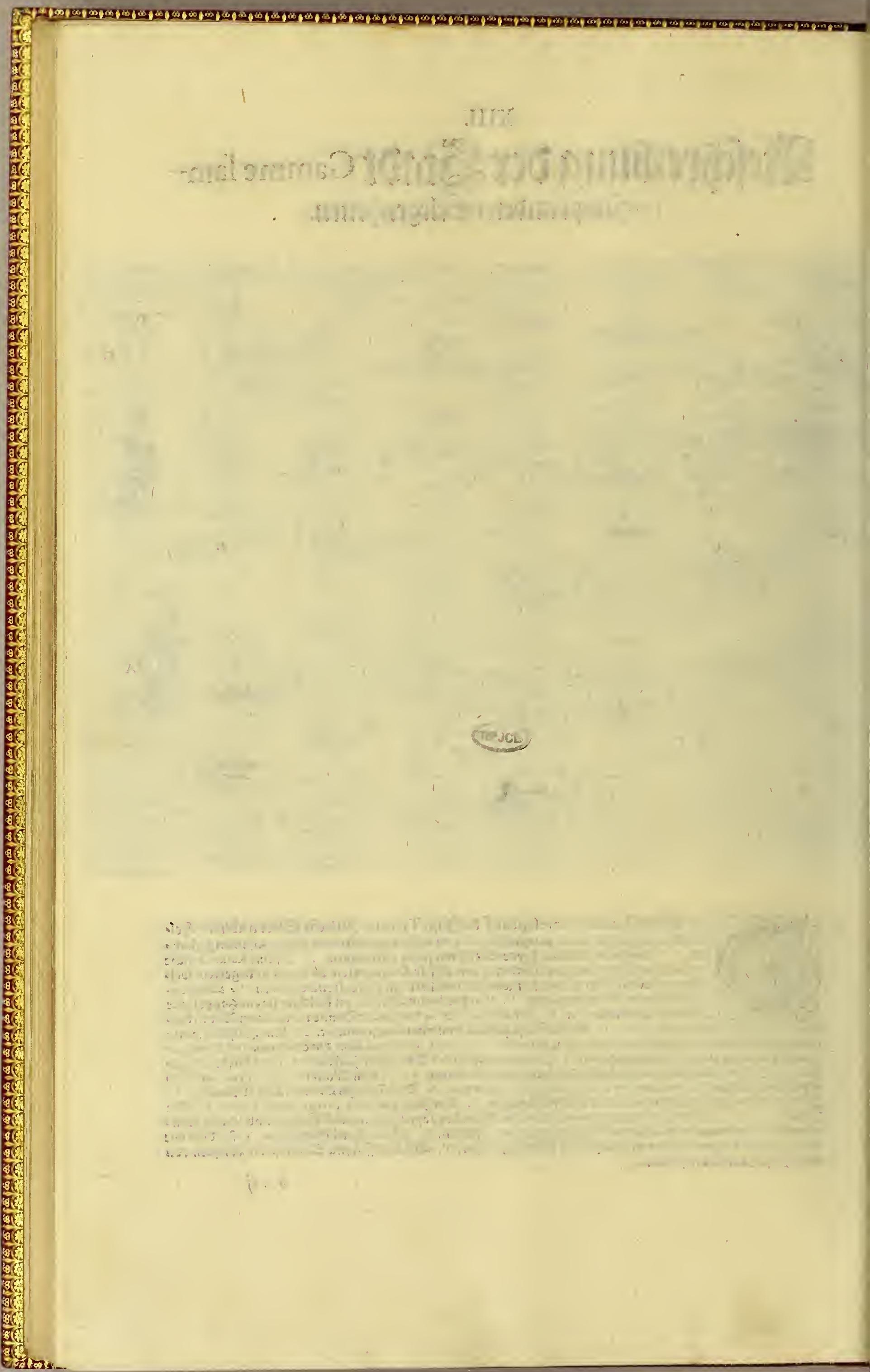




\section{XIV.

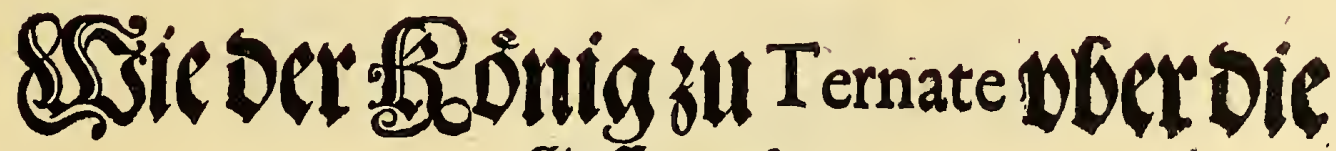 Gaflengetget.}

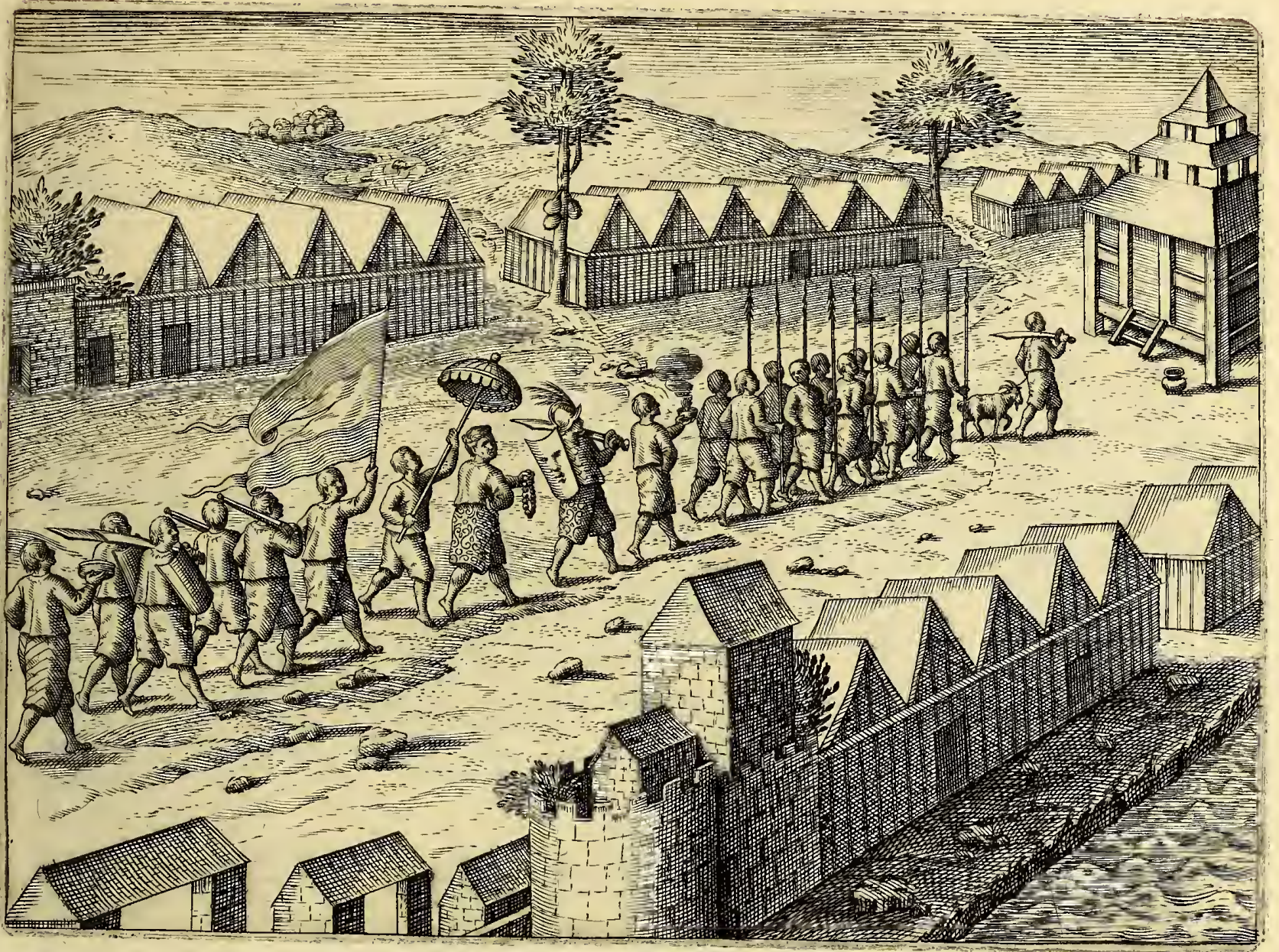

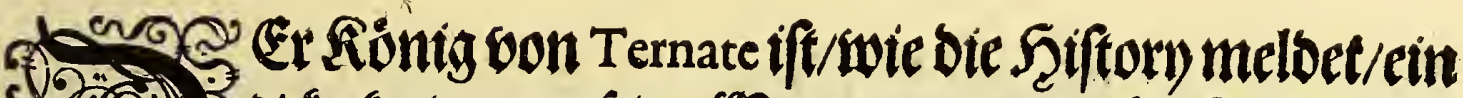

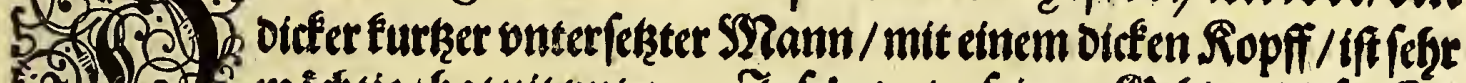

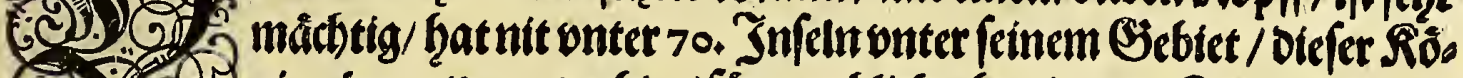

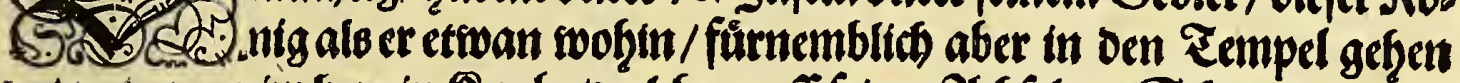

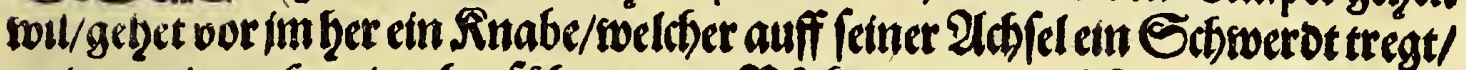

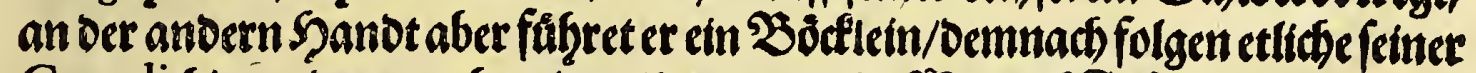

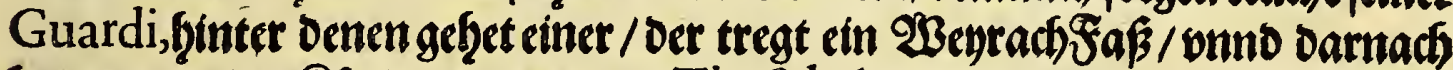

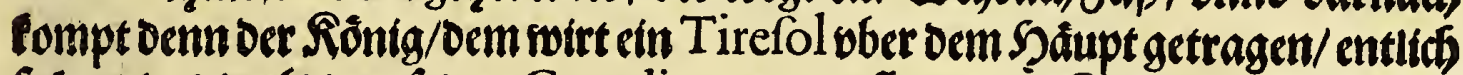

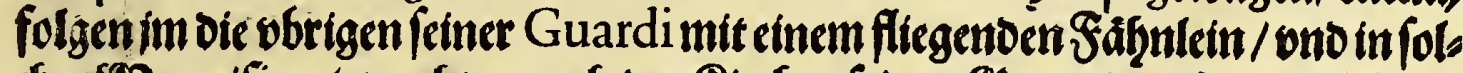

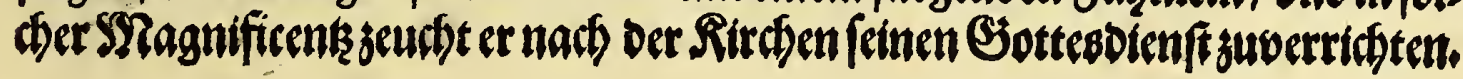

itif 


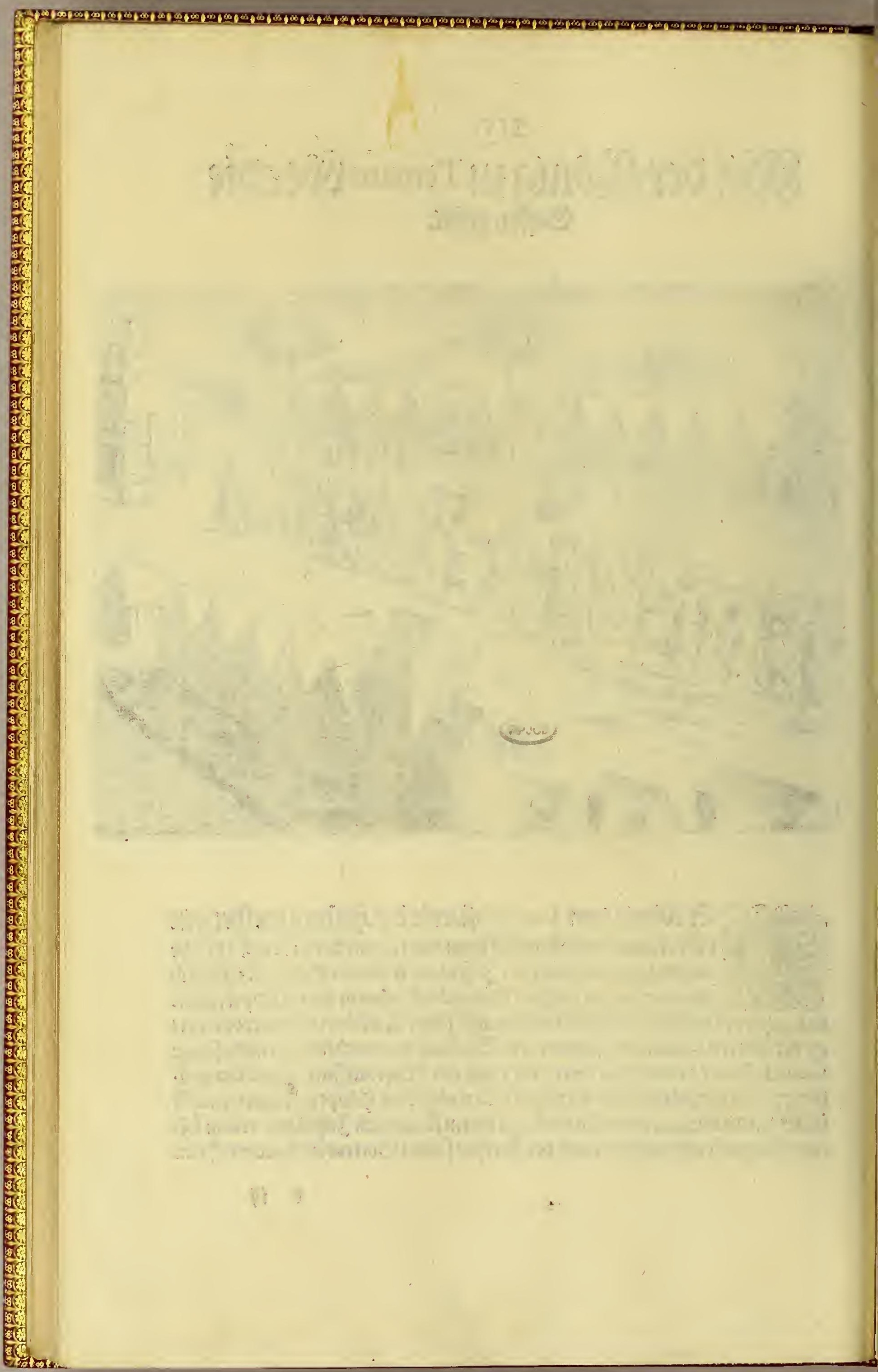




\section{XV. Fifecteter uno screciber in Moluco:}
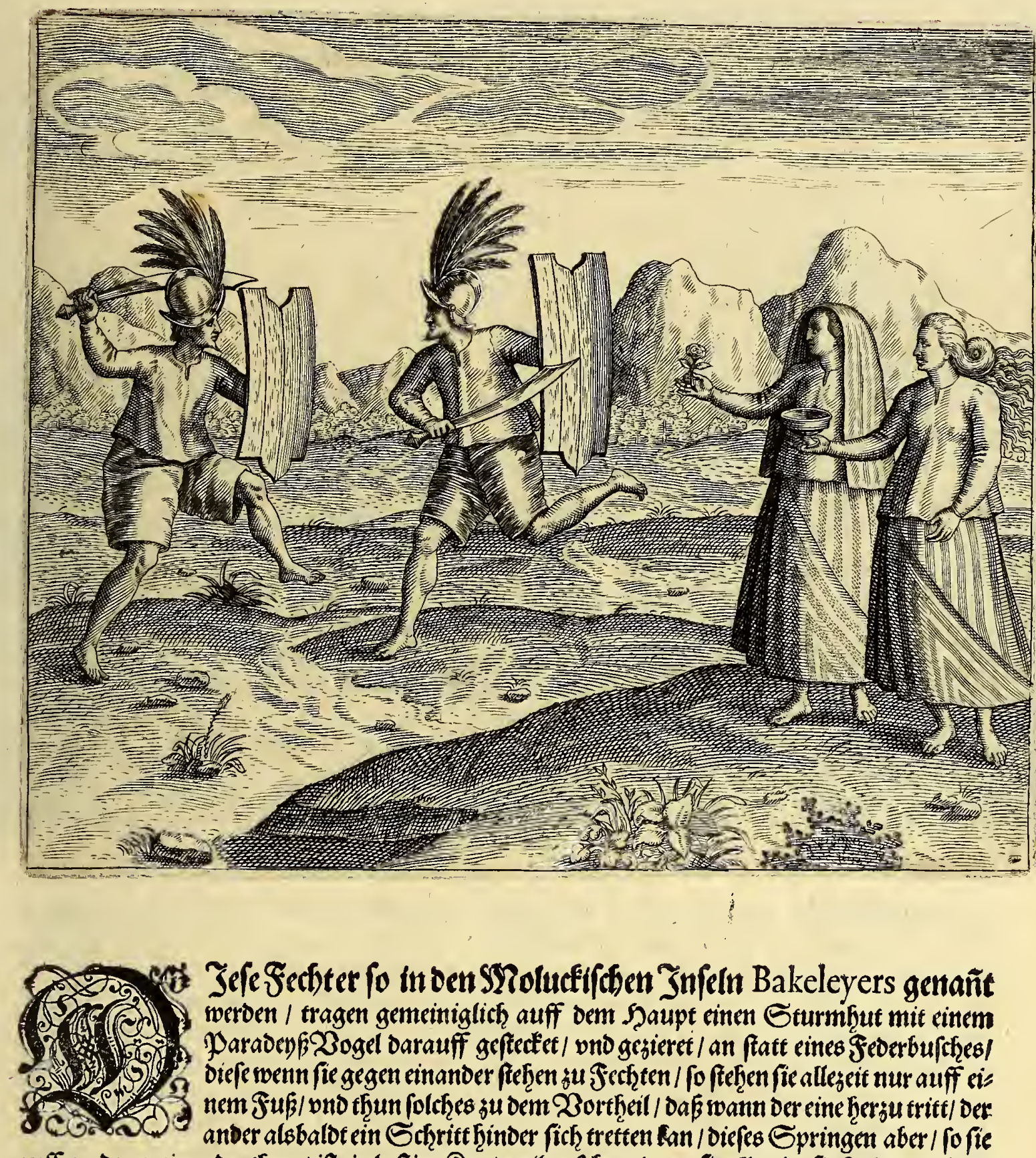

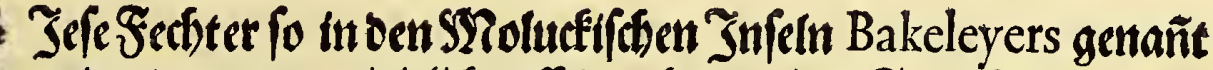
werben / tragen gemeiniglich auff dem Shaupt cinen Sturmfut mit einem

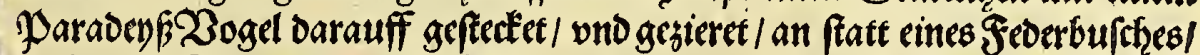

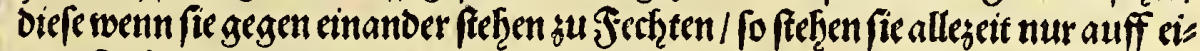

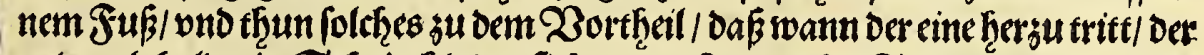
ander alsbalde ein Sefrzitt Finder fich tretten fan / Diefes Epringen aber / fo fie

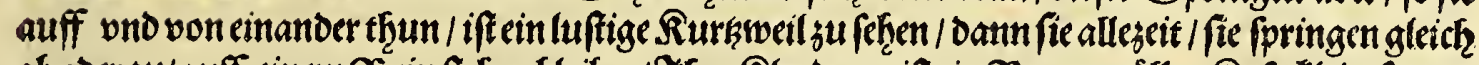

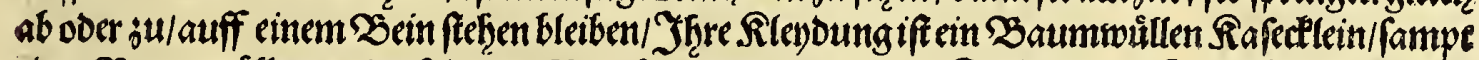

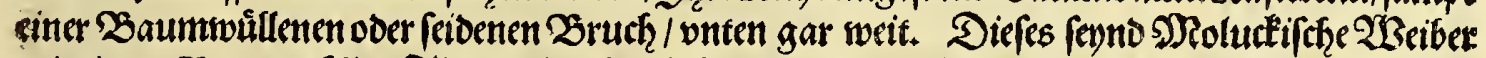

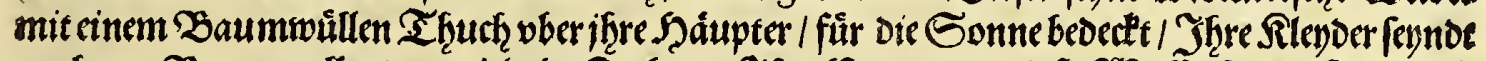

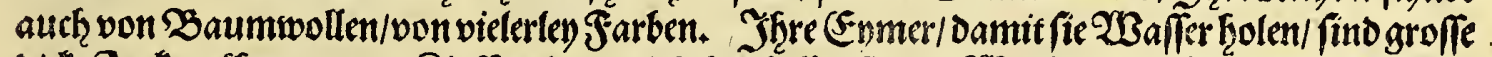

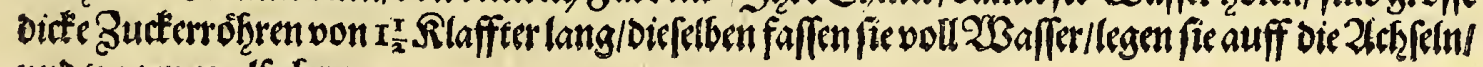
mend tragen es allo henm. 


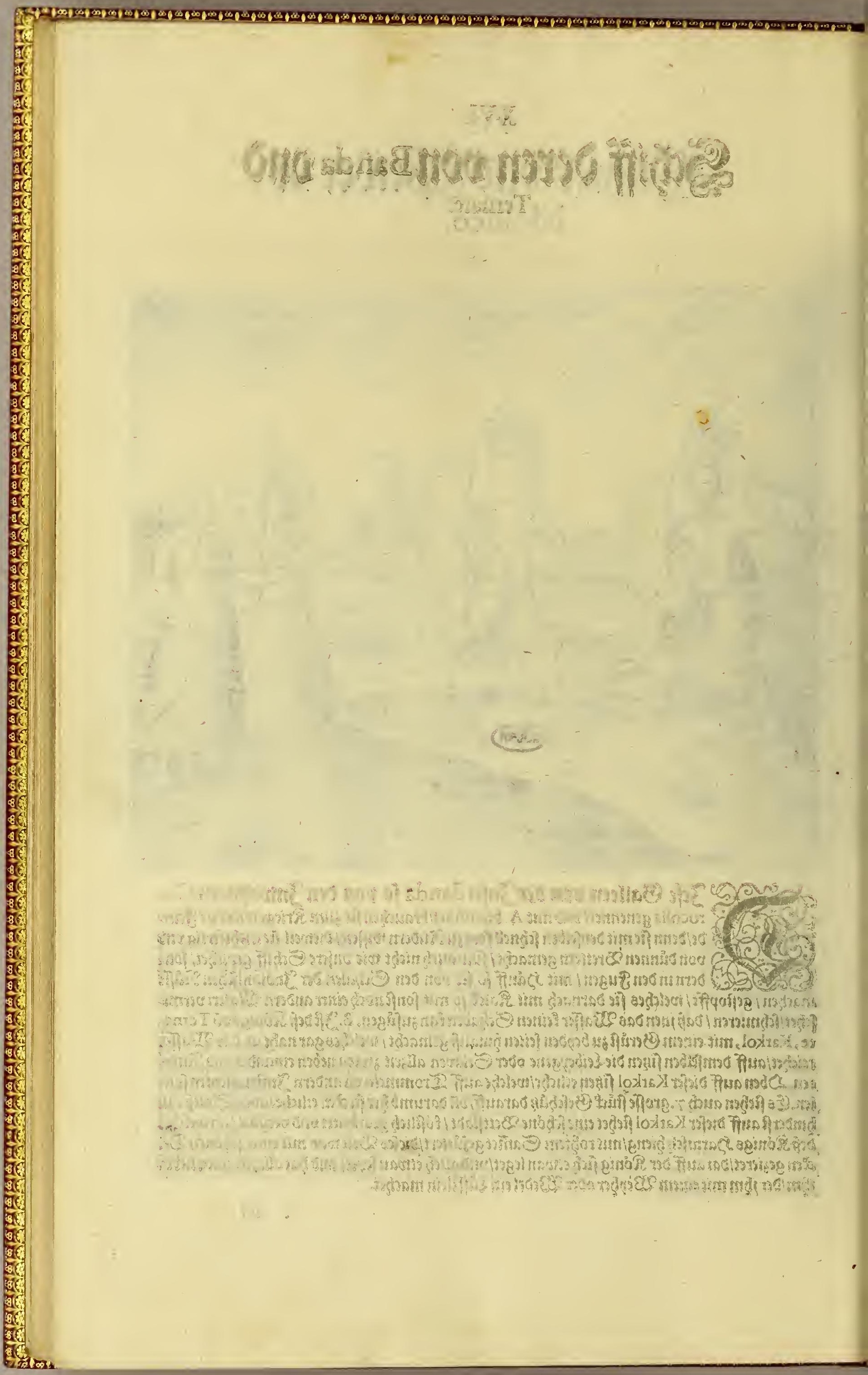




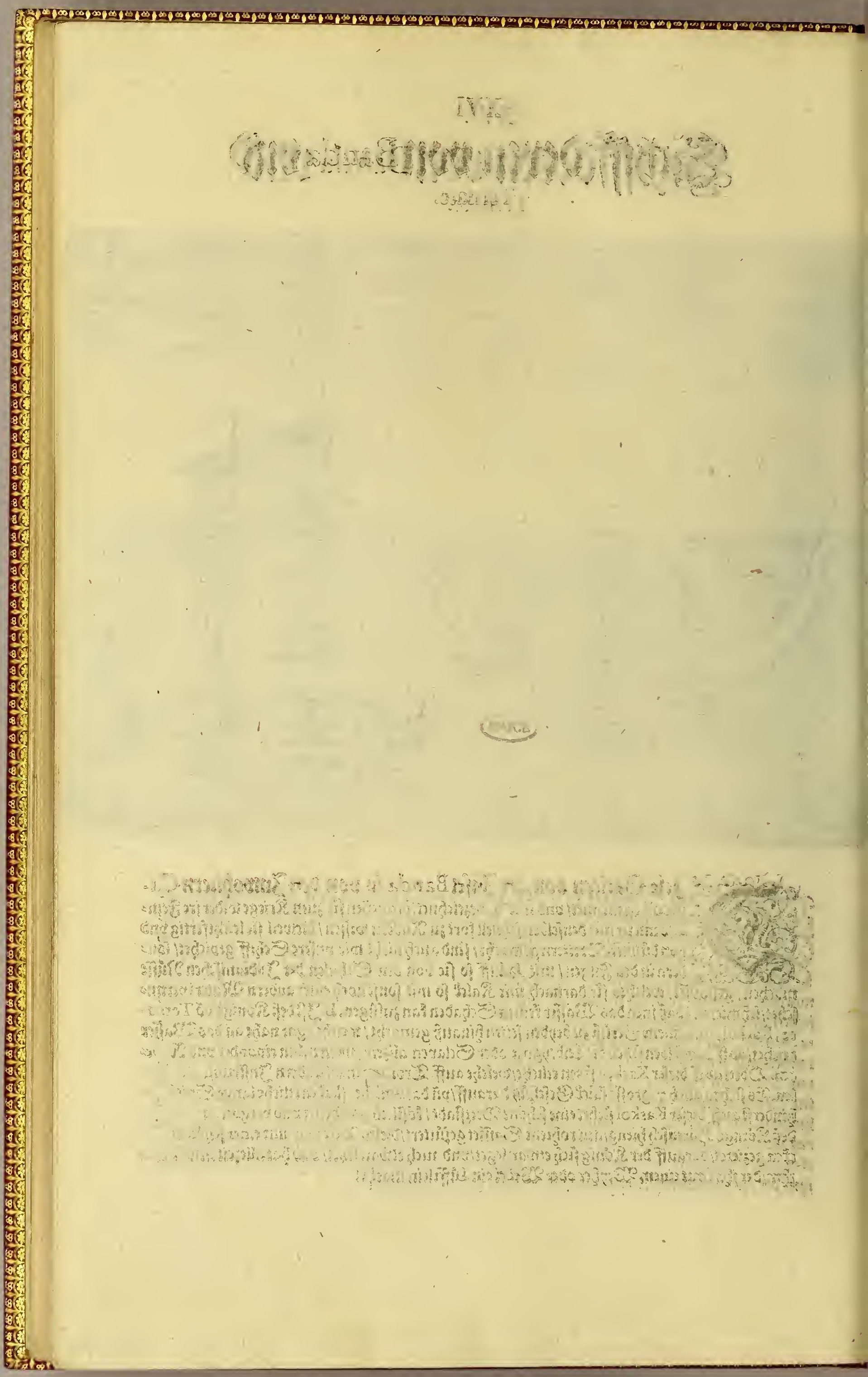




\section{XVII.}

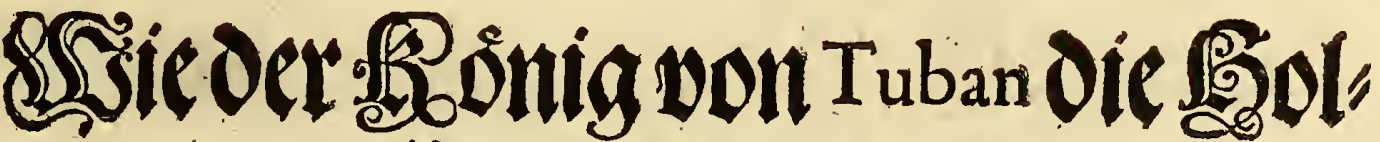 Ianter empfangen fiabe?}
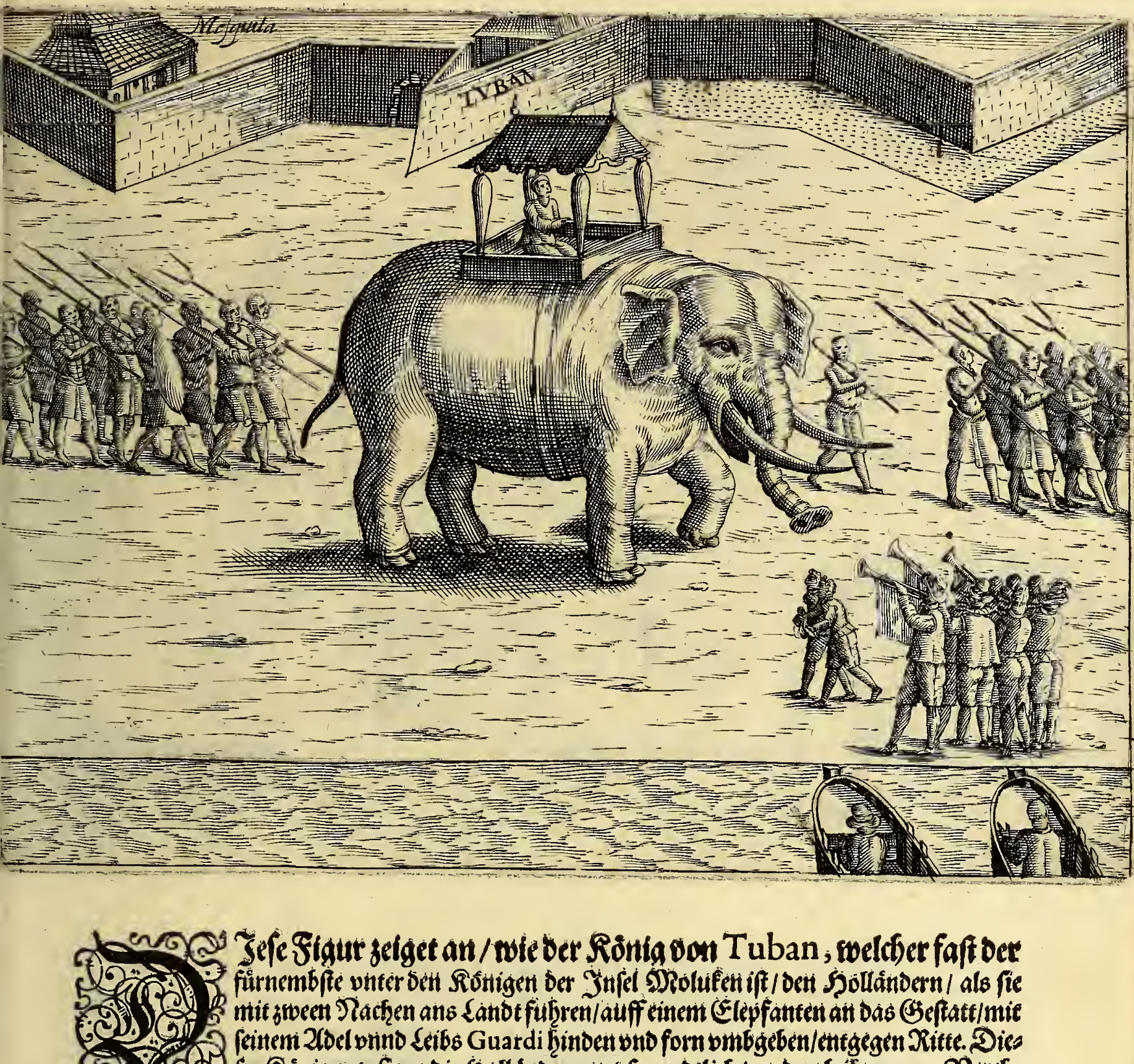

Sefe figur selget an / whe ber Rontia von Tuban, fwelder faft ber

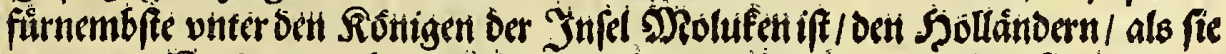

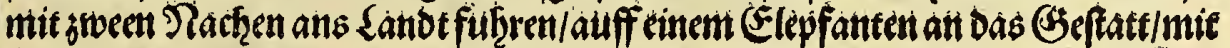

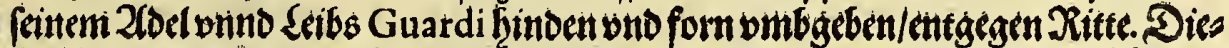

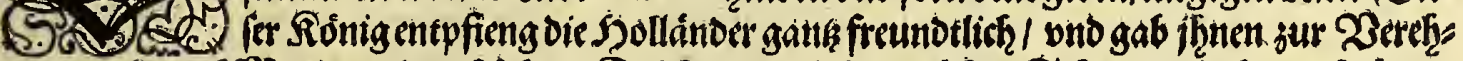

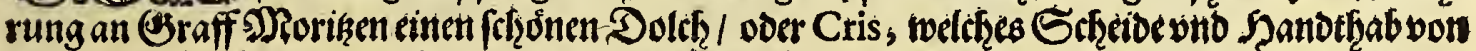

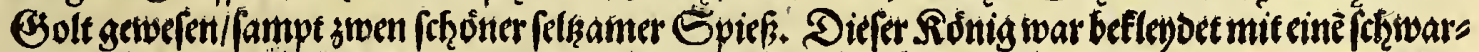

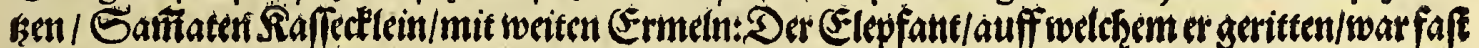

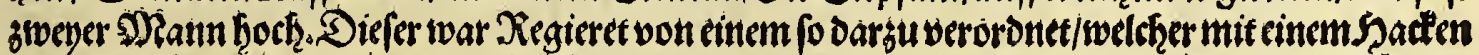

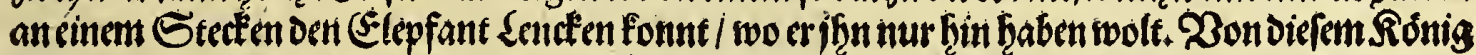

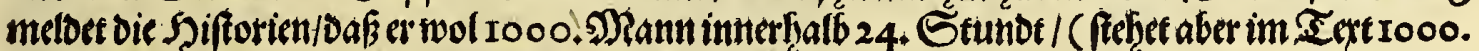

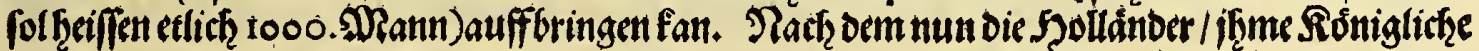

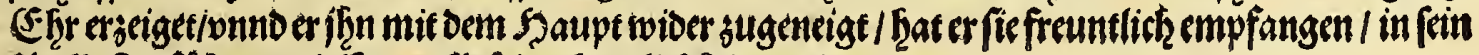

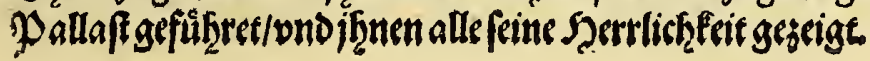




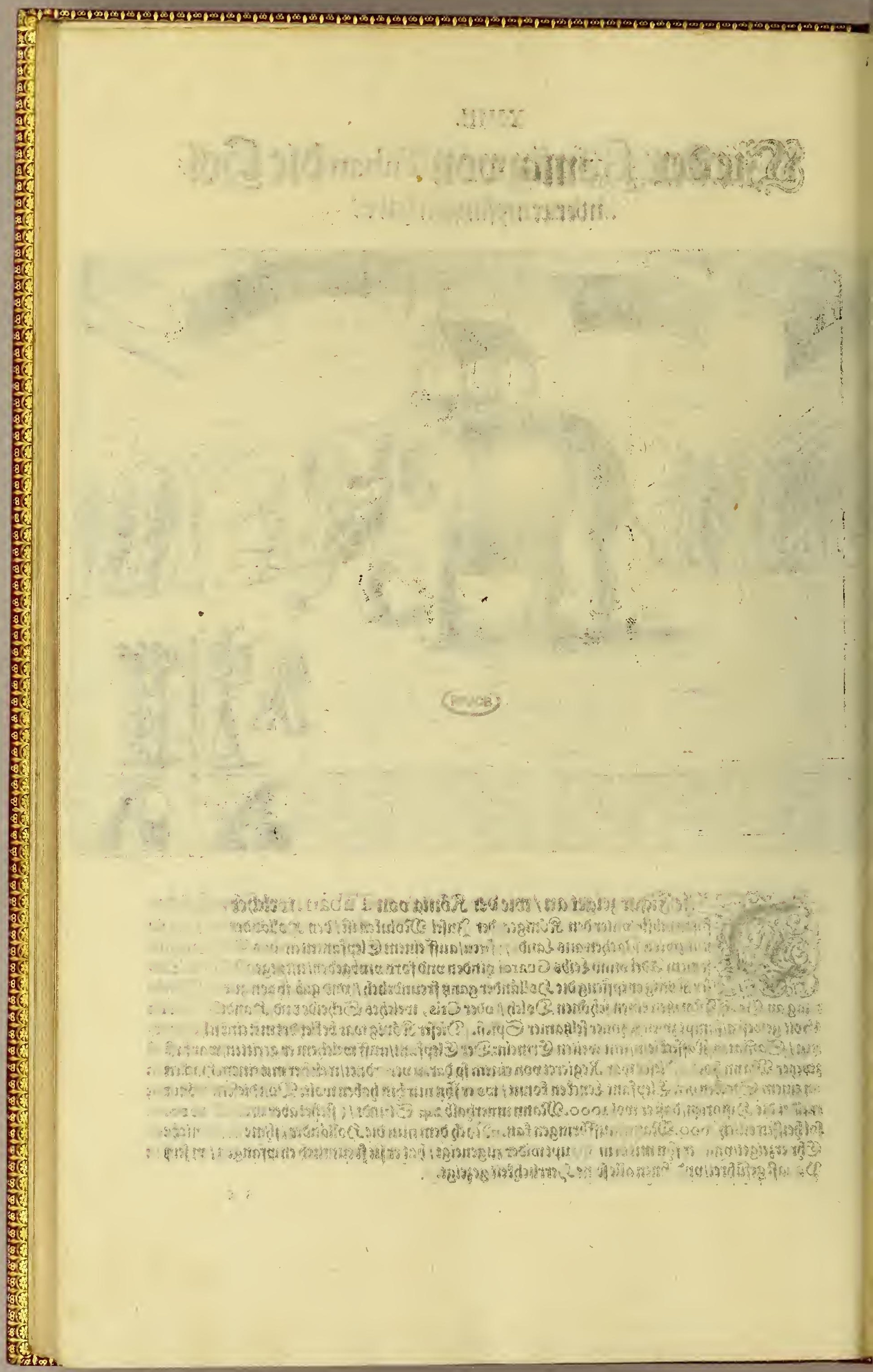




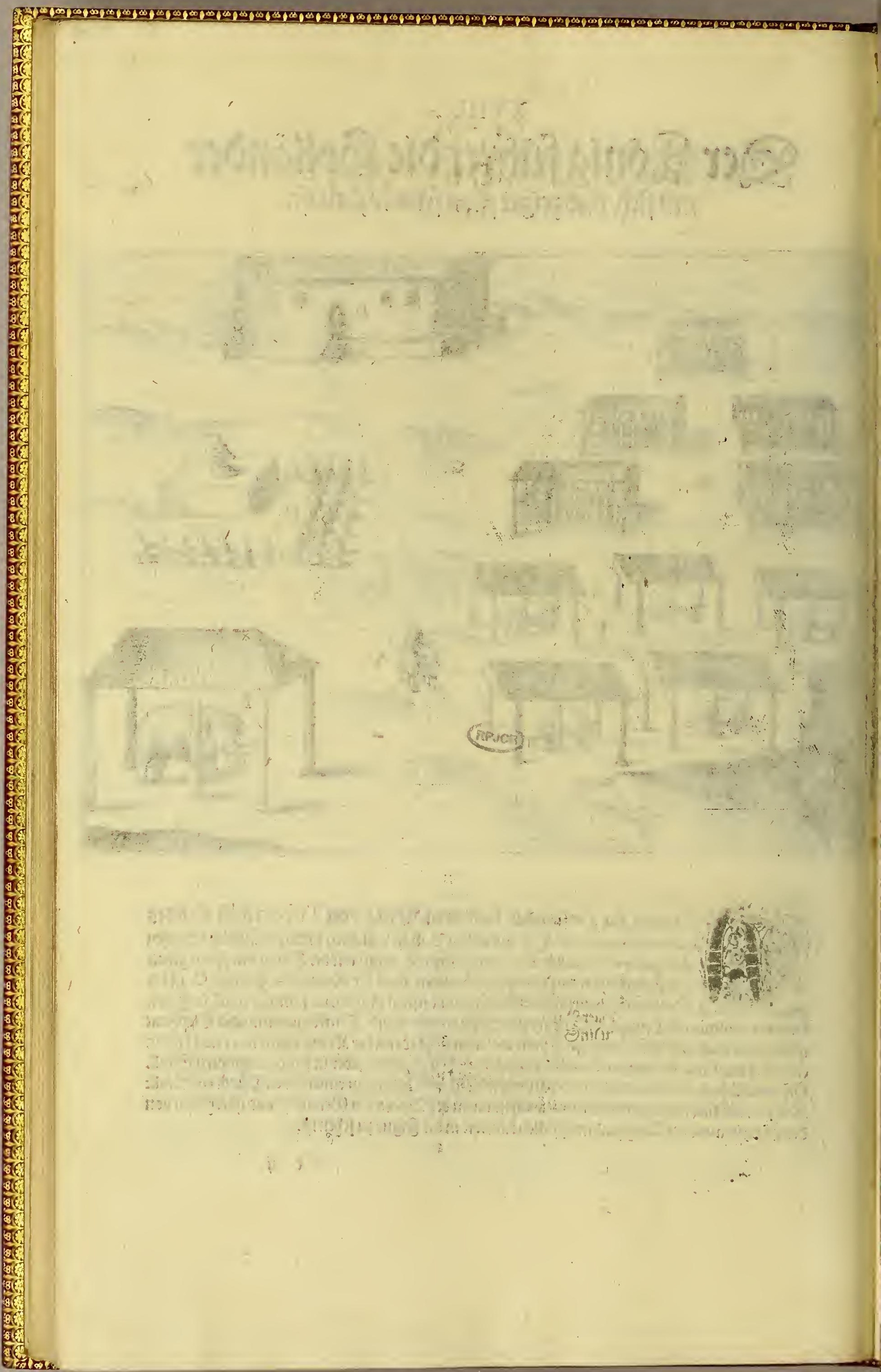




\section{XIX.

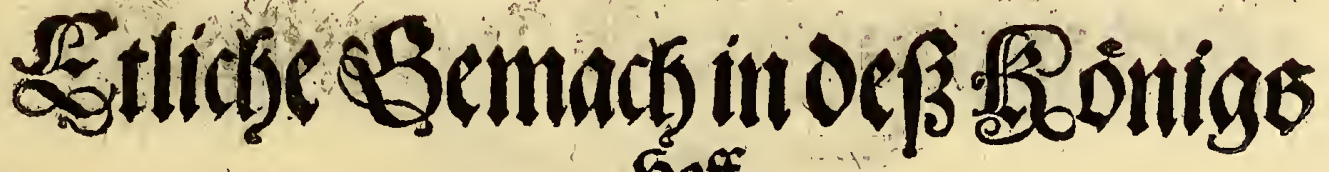 গ⿺辶f}
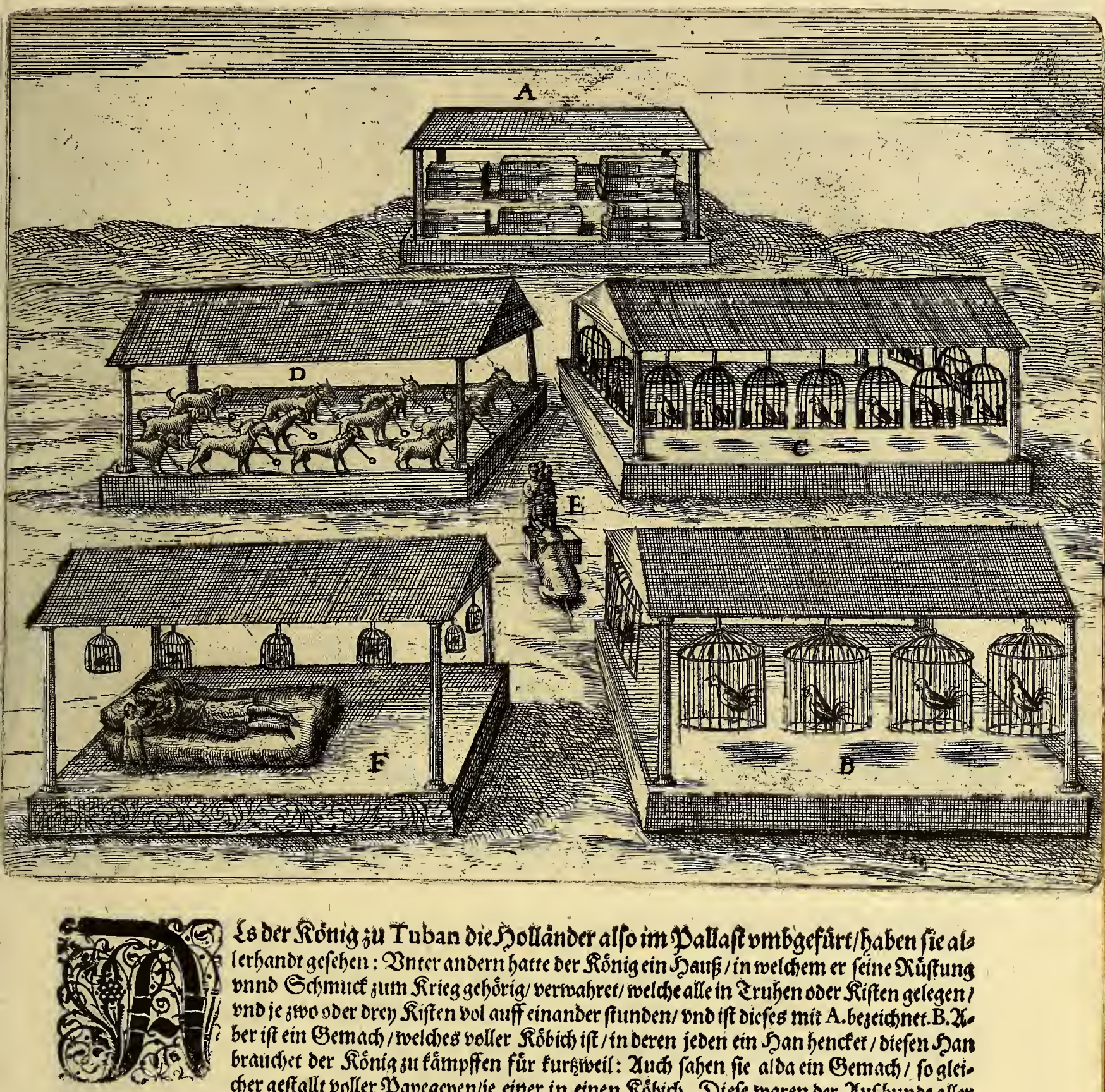

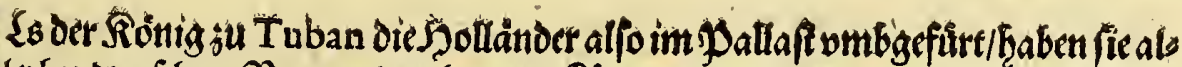

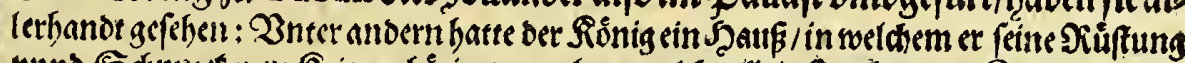

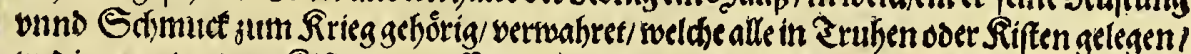

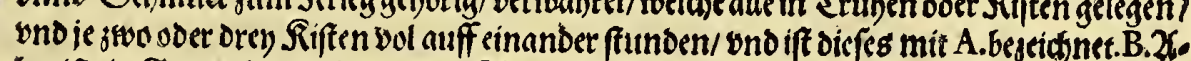

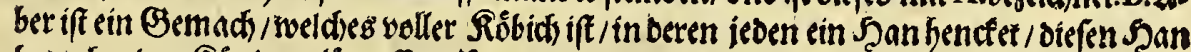

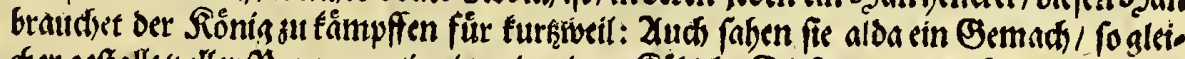

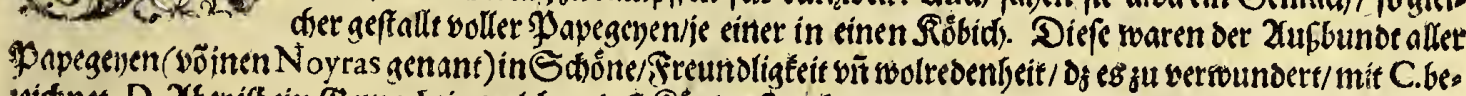

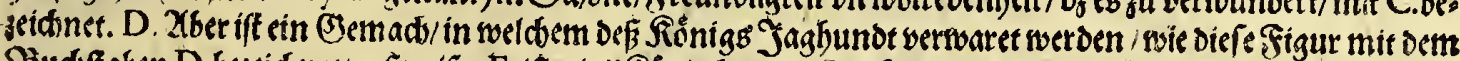

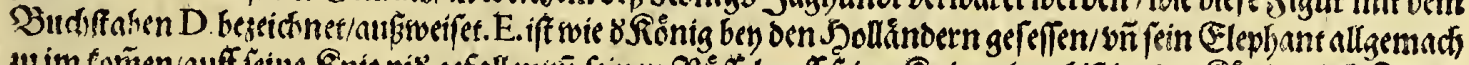

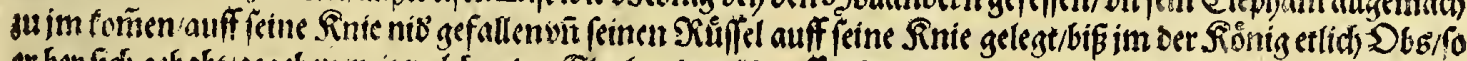

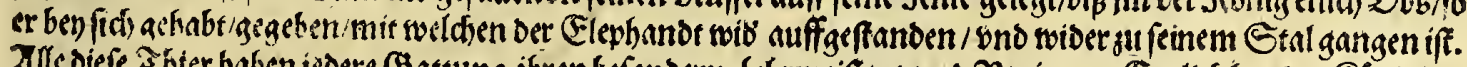

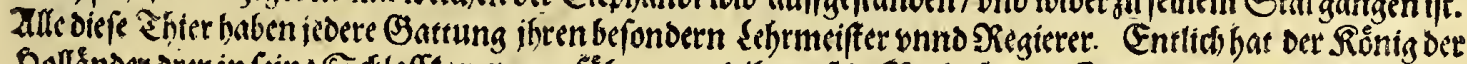

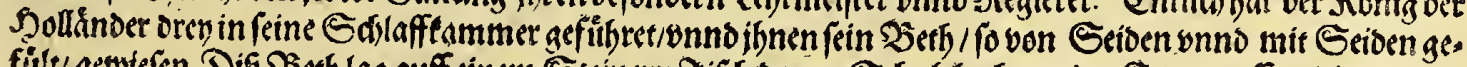

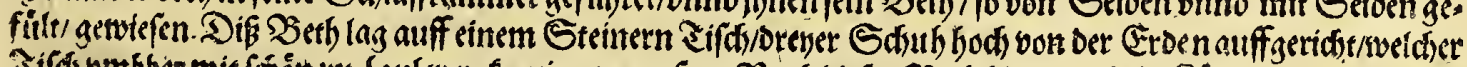

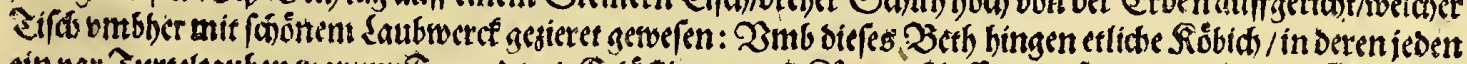

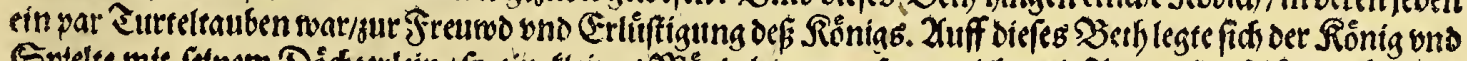

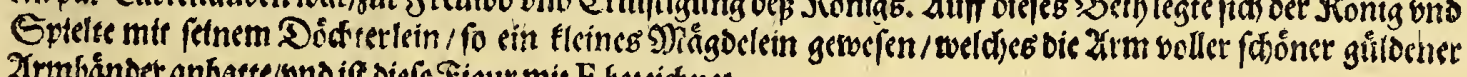
Aatmbaindet anbatte/bnd ift diefe Stigur mit F. beseidinct. 


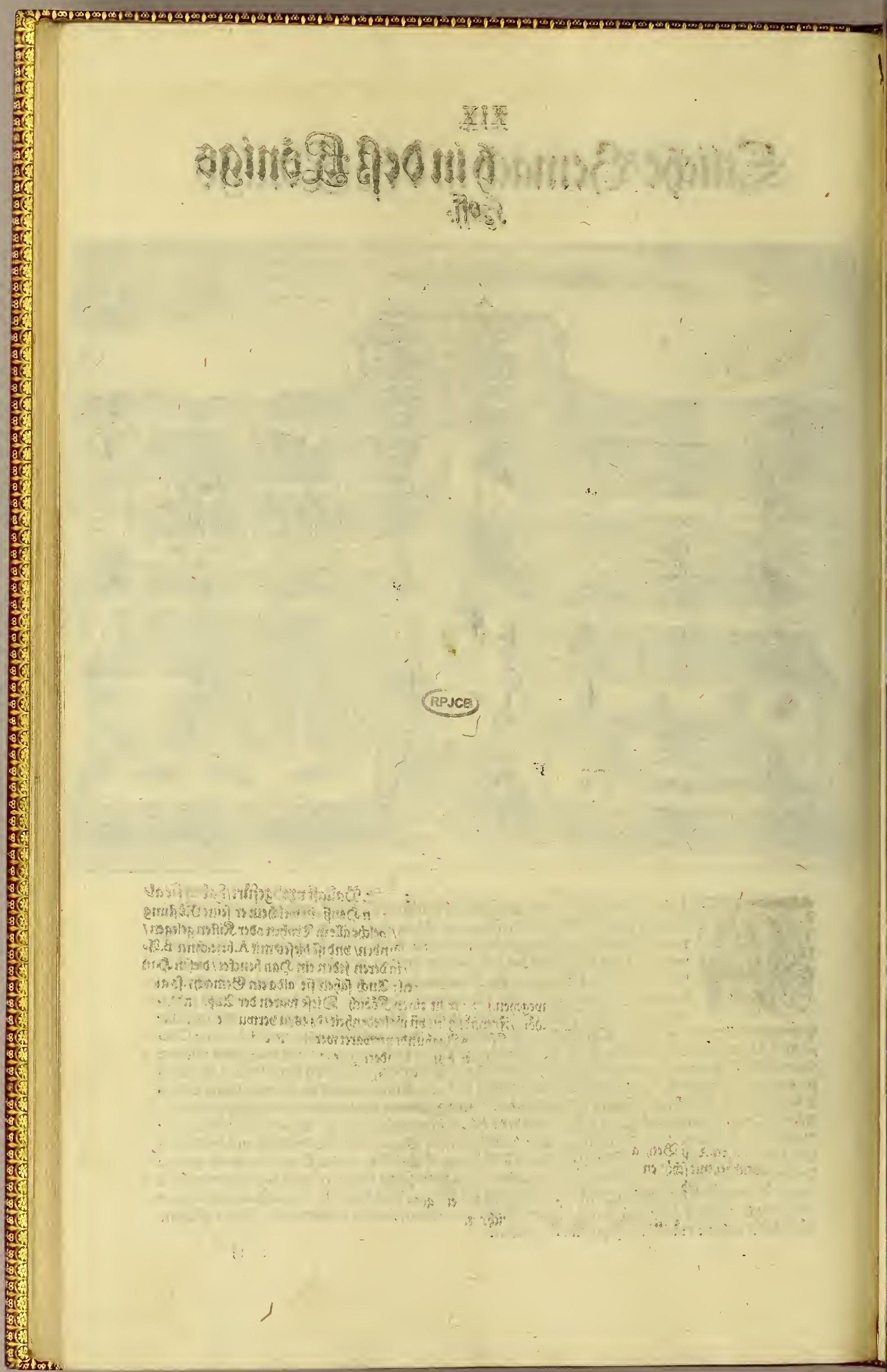




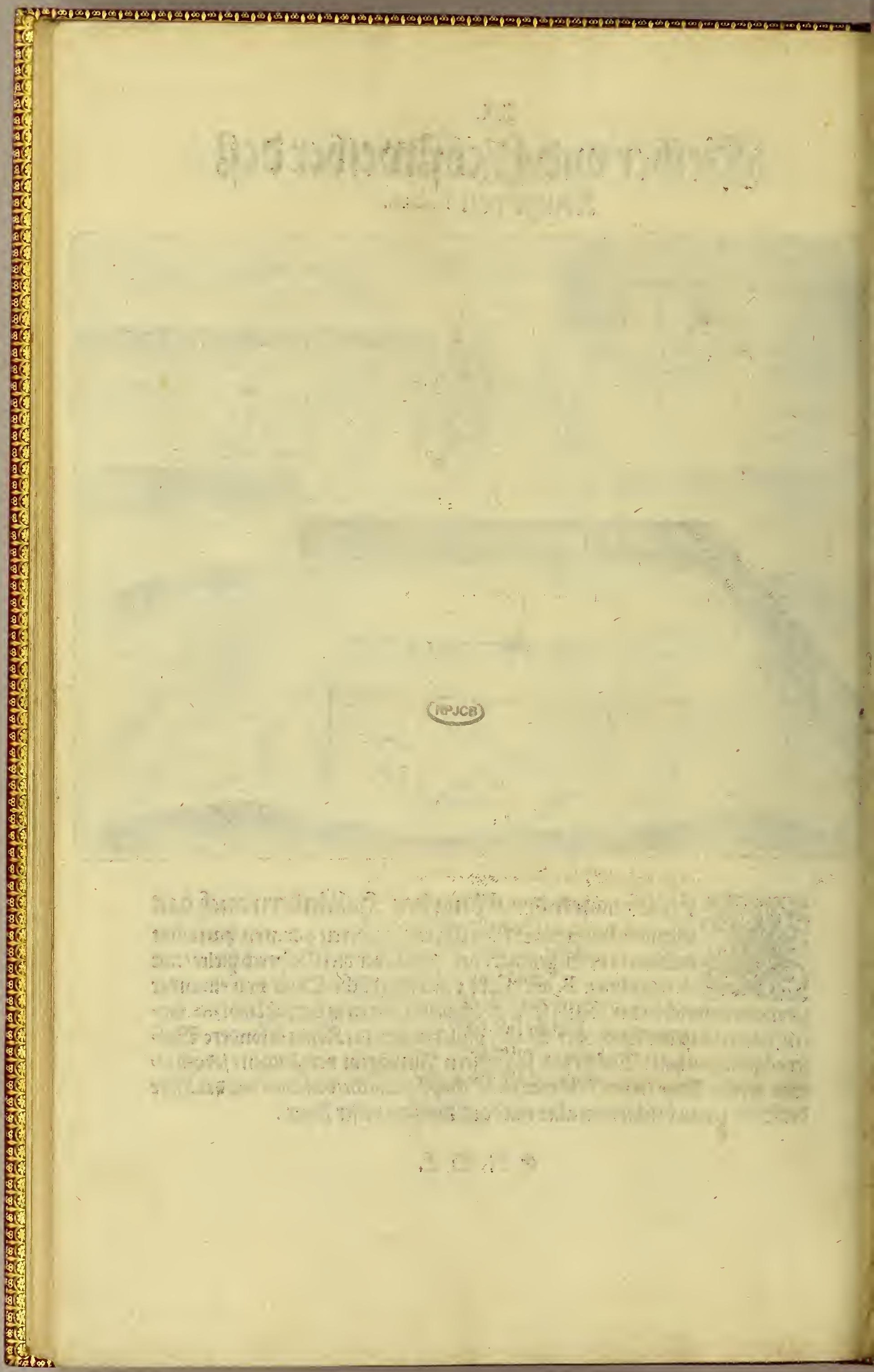




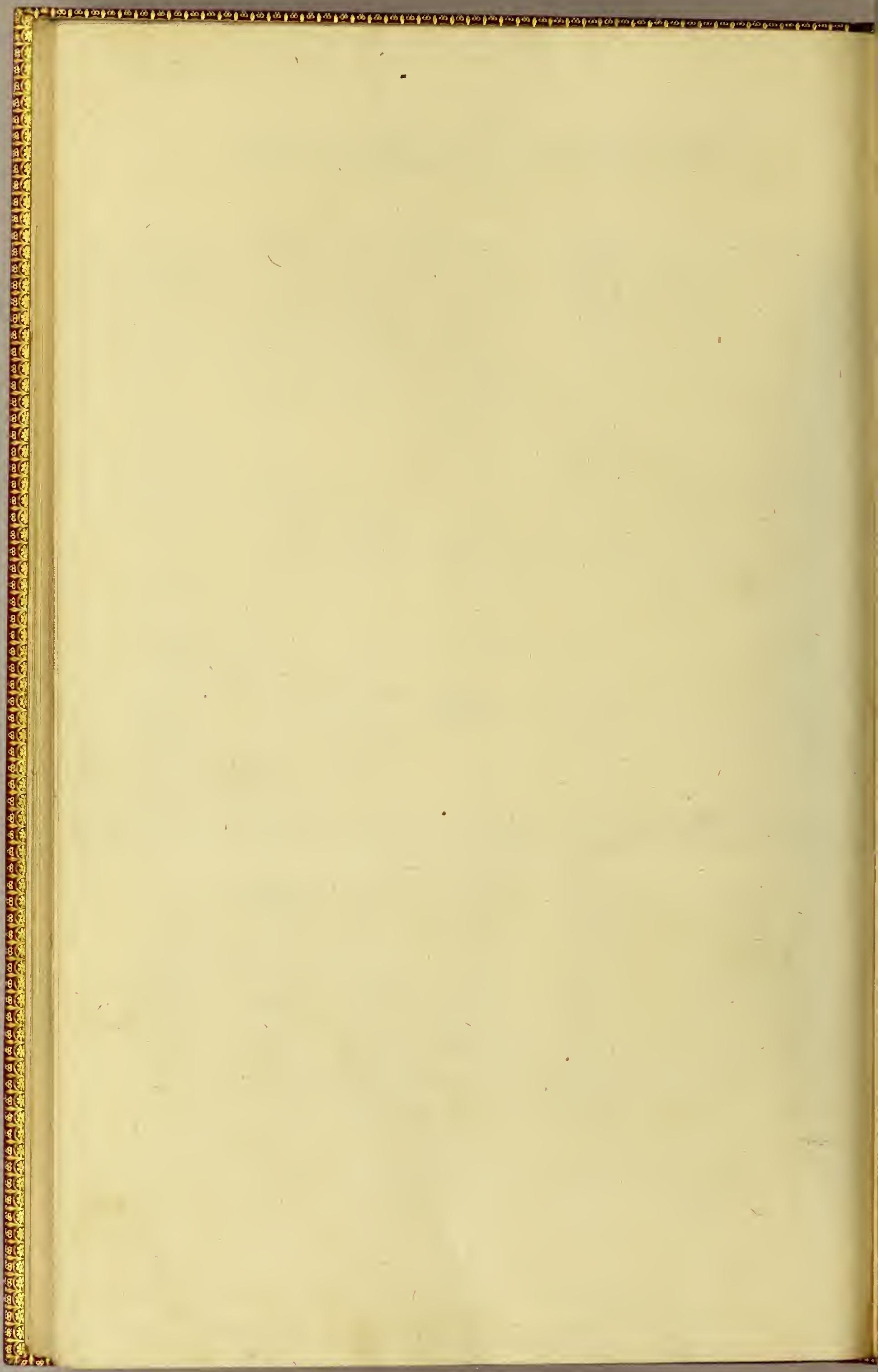




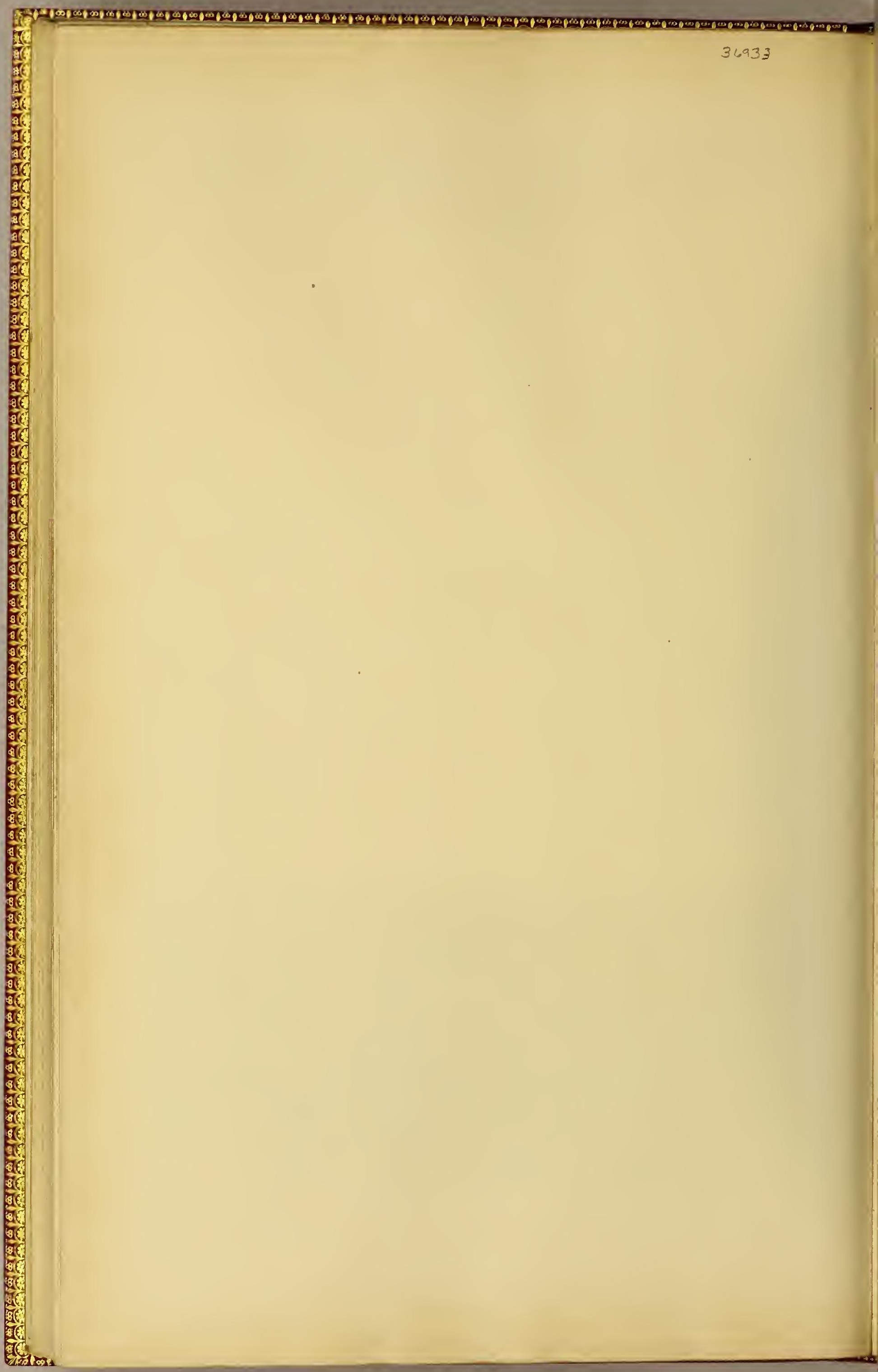





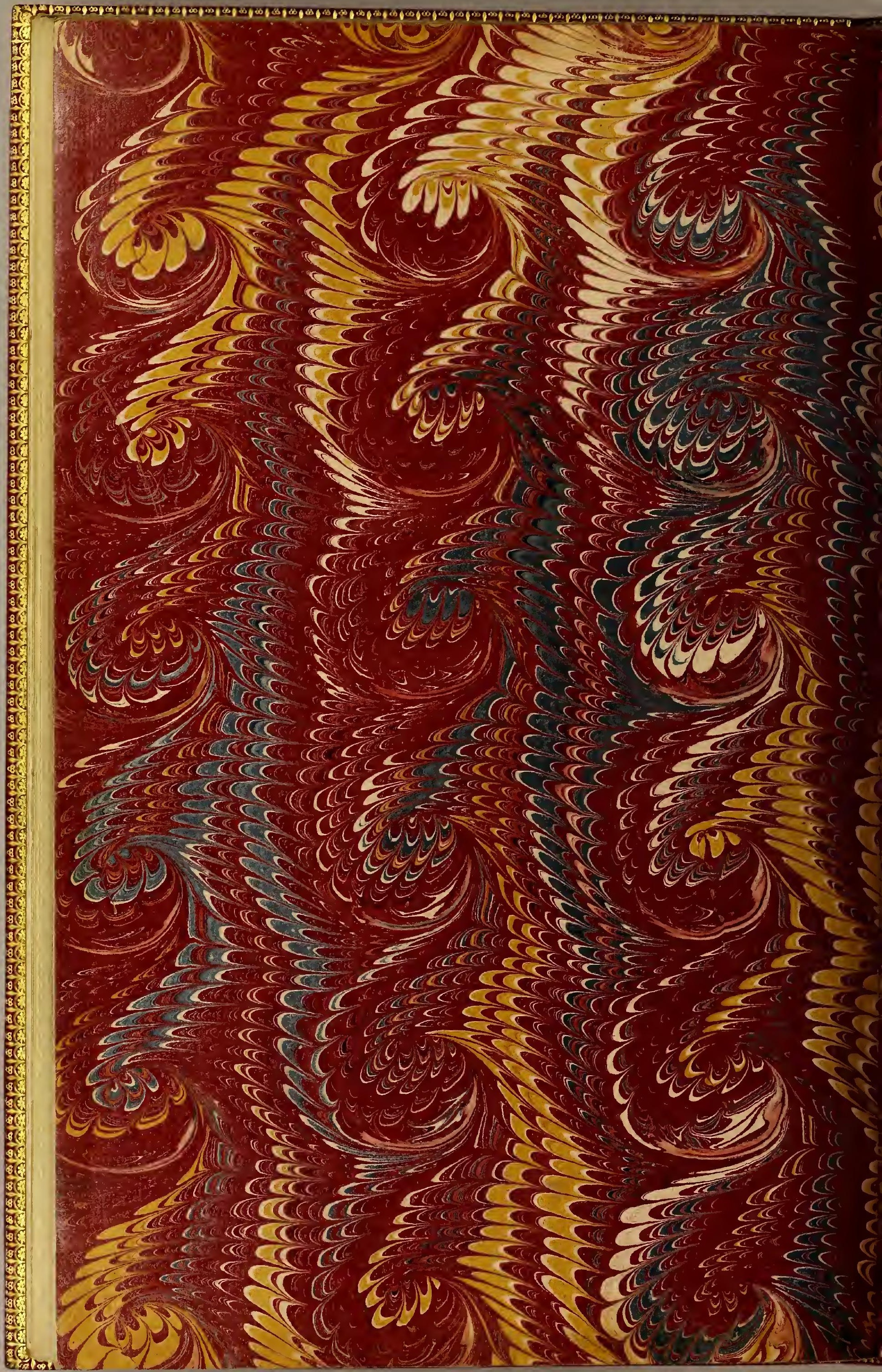




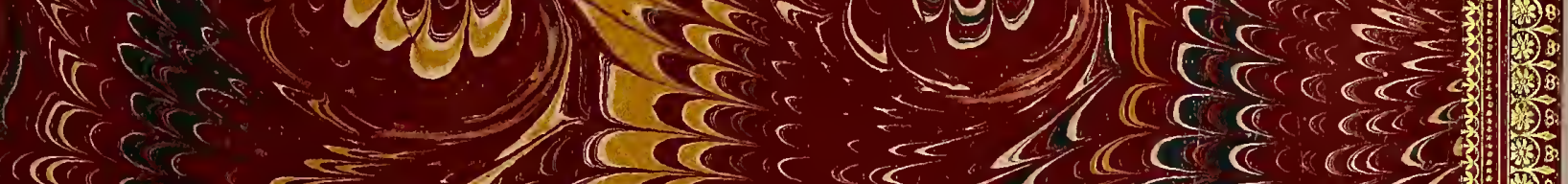

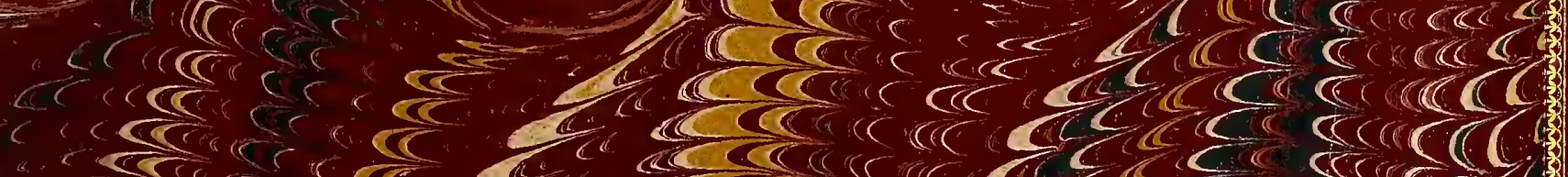

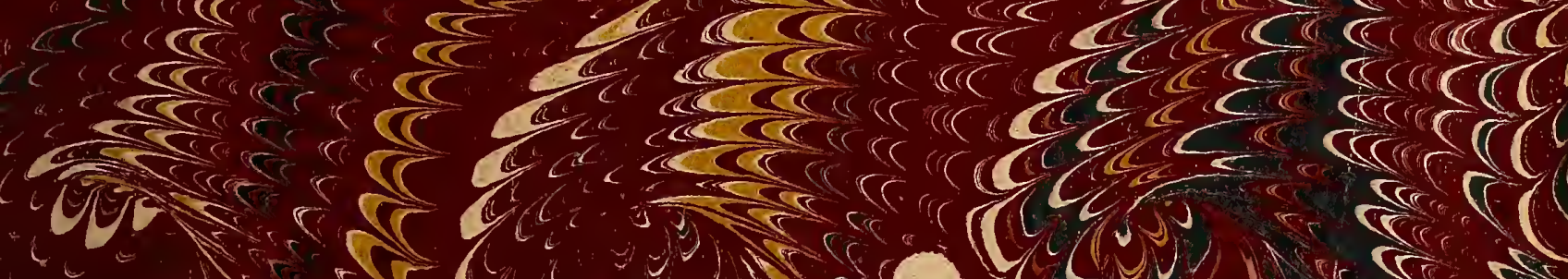

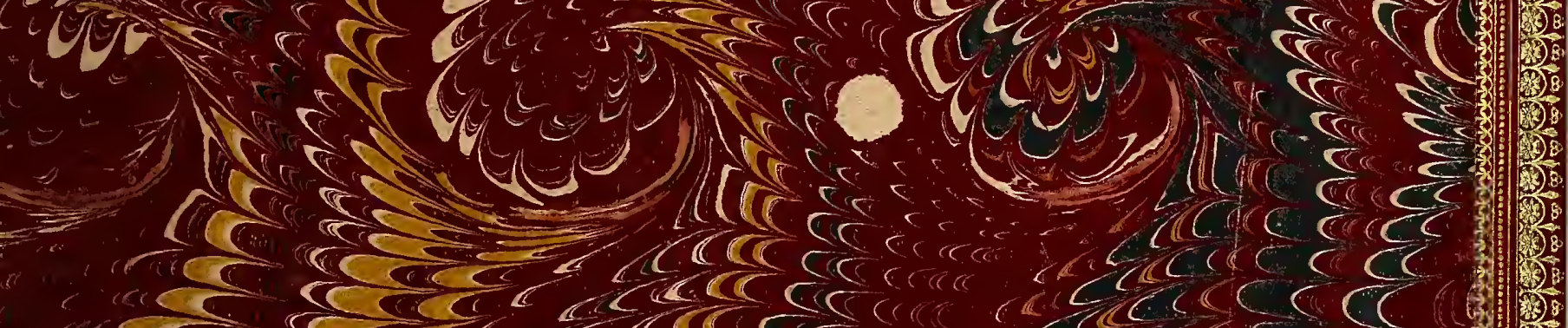

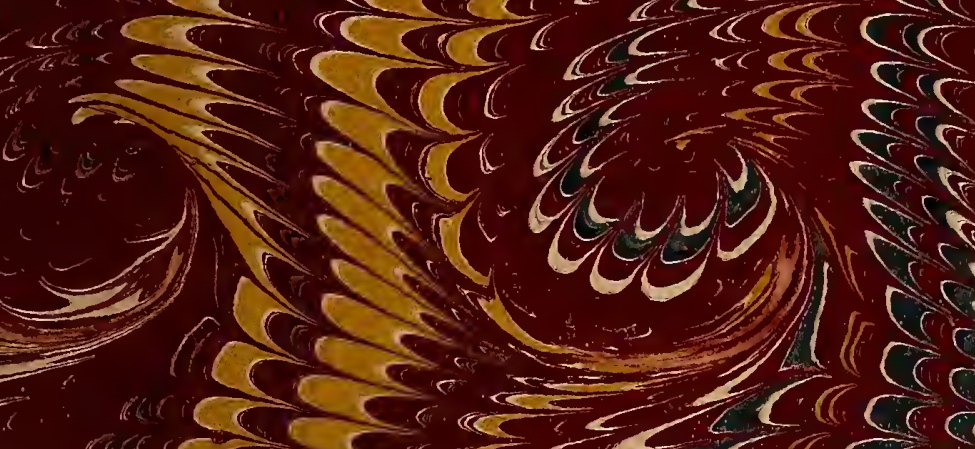

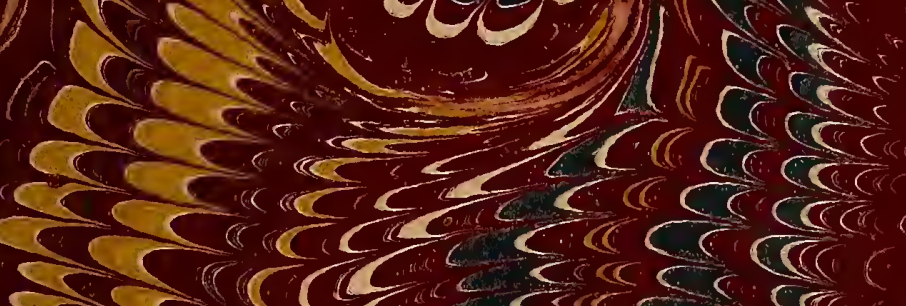

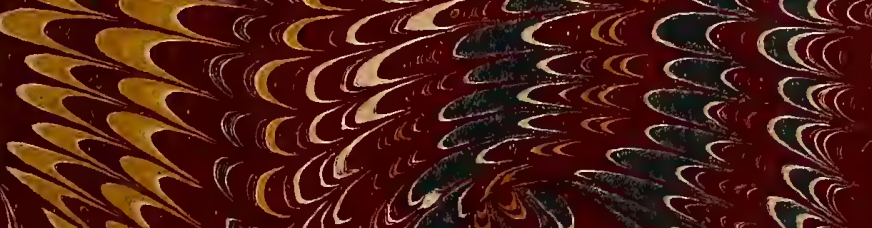

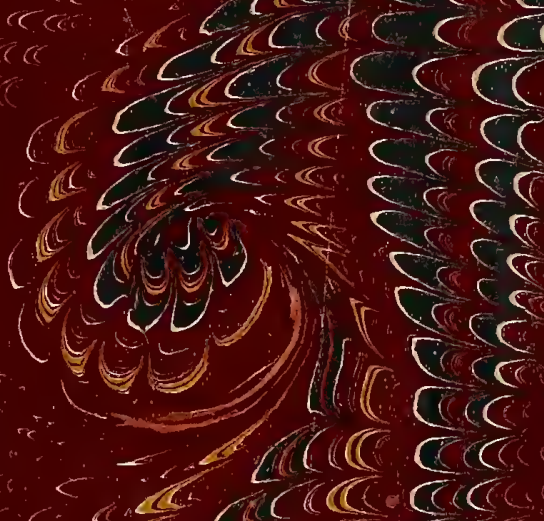

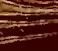

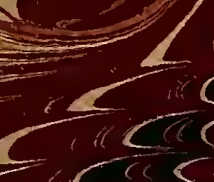

-20

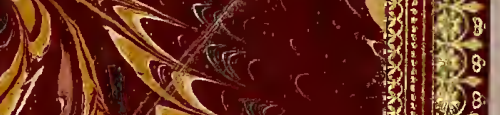

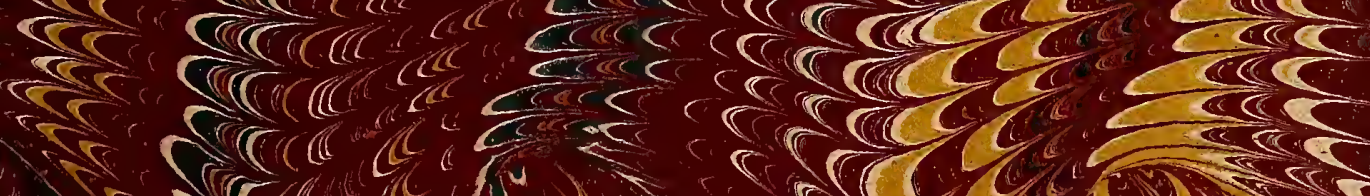

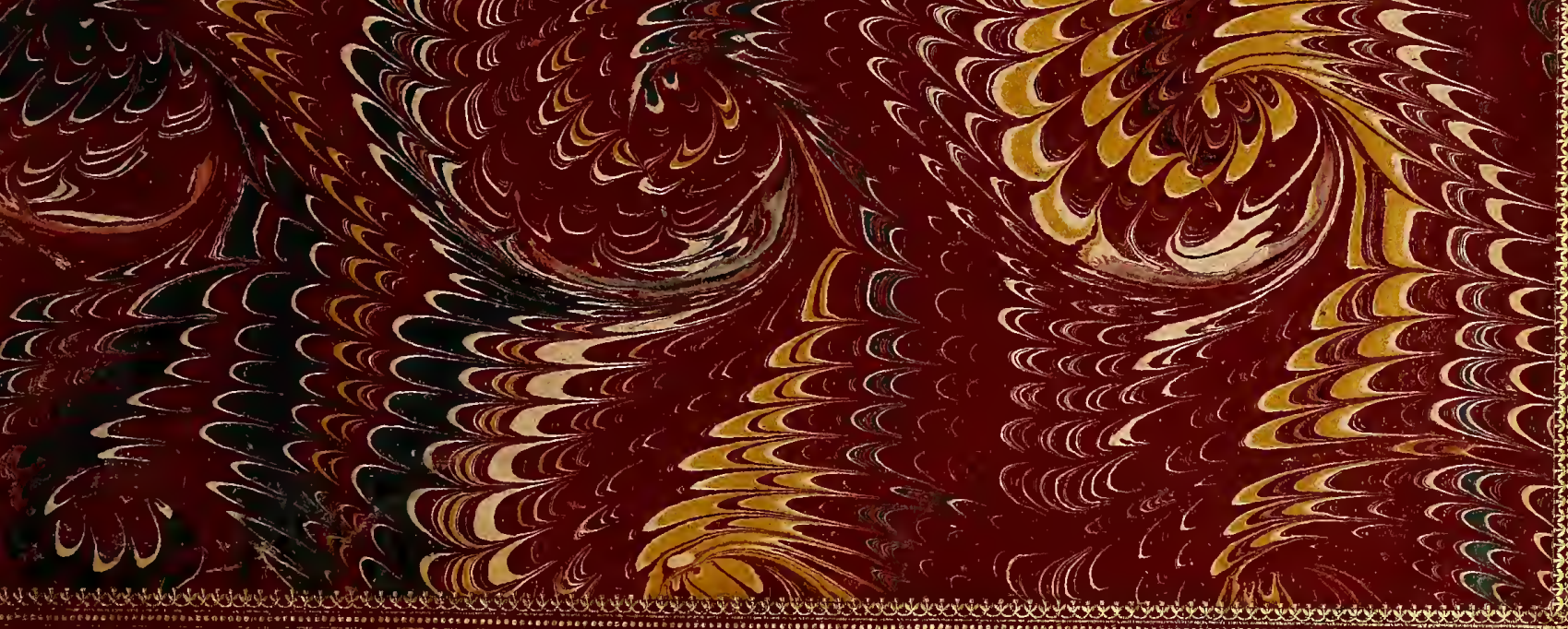

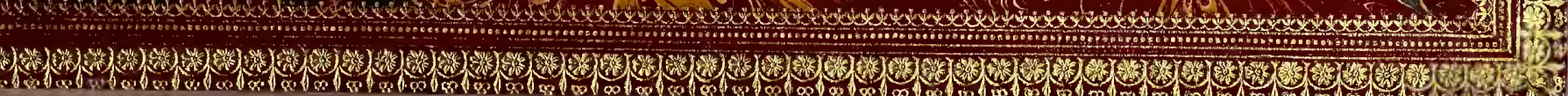




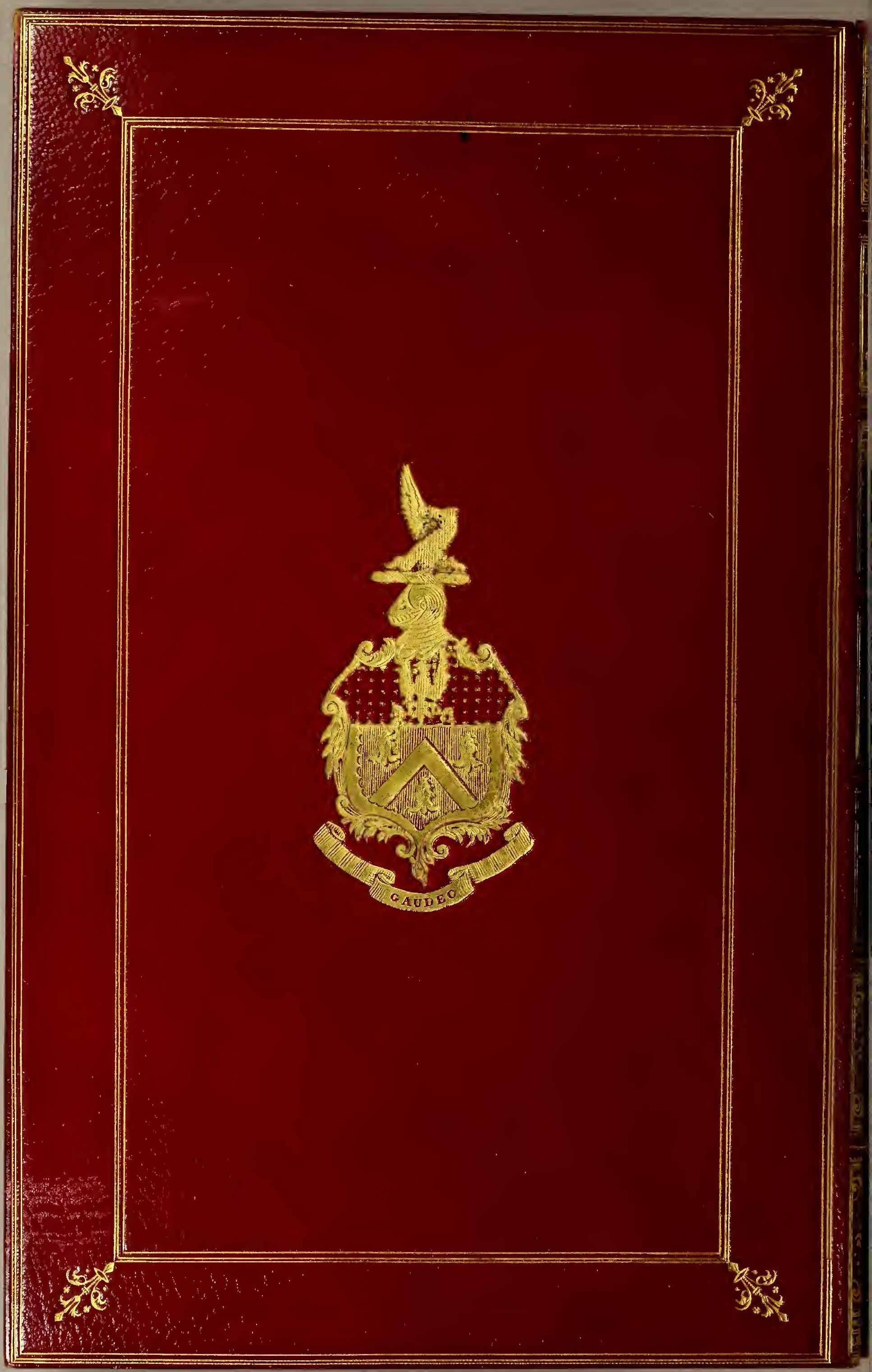

